\title{
Radionuclide Cycling in A Utah Dairy Farm
}

January 1976

Prepared for the U.S. Energy

Research and Development Administration under Contract E(45-1):1830 
NOTICE

This report was prepared as an account of work sponsored by the United States Government. Neither the United States nor the United States Energy Research and Development Administration, nor any of their employees, nor any of their contractors, subcontractors, or their employees, makes any warranty, express or implied, or assumes any legal liability or responsibility for the accuracy, completeness or usefulness of any information, apparatus, product or process disclosed, or represents that its use would not infringe privately owned rights.

PACIFIC NORTHWEST LABORATORY

operated by

BATTELLE

for the

U.S. ENERGY RESEARCH AND DEVELOPMENT ADMINISTRATION

Under Contract $E(45-1)-1830$

Printed in the United States of America Available from

National Technical tnformation Service

U.S. Department of Commerce

5285 Port Royal Road

Springfield, Virginia 22151

Price: Printed Copy \$9.00; Microfiche \$2.25 


\title{
Radionuclide Cycling in a Utah Dairy Farm
}

\author{
J. M. Thomas and L. L. Eberhardt \\ Battelle \\ Pacific Northwest Laboratory \\ Richland, Washington 99352 \\ H. A. Hawthorne and S. D. Zellmer \\ Laboratory of Nuclear Medicine and \\ Radiation Biology \\ University of California \\ Los Angeles, California 90024
}

January 1976

Battelle Pacific Northwest Laboratories Richland, Washington 99352 


\section{CONTENTS}

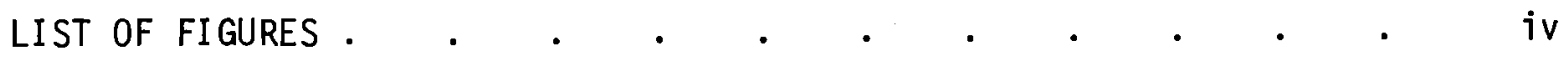

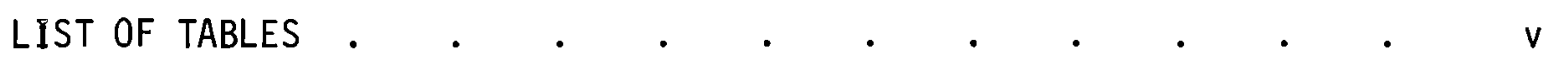
LIST OF TABLES IN APPENDIX A LIST OF FIGURES IN APPENDIX B $B$.

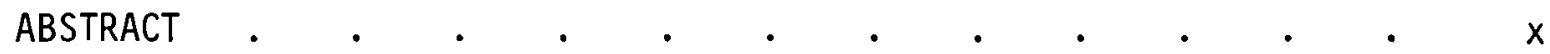
1.0 INTRODUCTION

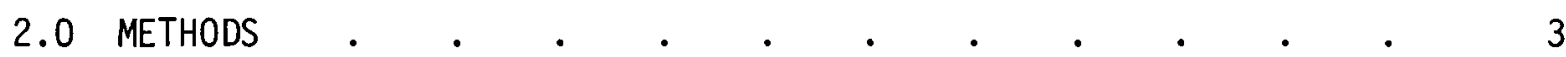

3.0 PREVIOUS MATHEMATICAL MODELS

4.0 UTAH MODELS AND ANALYSIS OF DATA $\quad$.

4.1 POT AND FUNNEL COLLECTORS • . . . . . . . . . 6

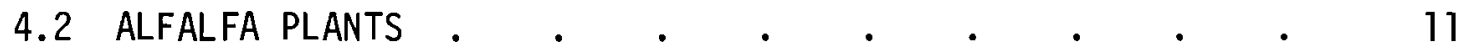

4.3 GROWTH CHAMBER STUDY . . . . . . . . . . . . . . 20

4.4 SOILS AND IRRIGATION WATER.$\quad \cdot \quad \cdot \quad \cdot \quad \cdot \quad \cdot \quad 21$

4.5 TESTS FOR LINEARITY

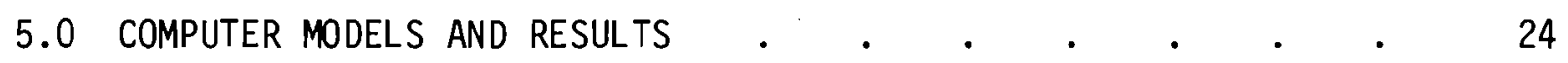

5.1 GENERAL DESCRIPTION

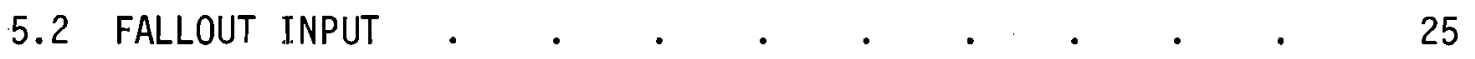

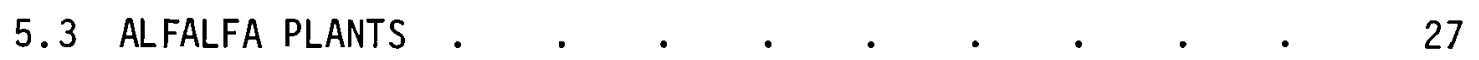

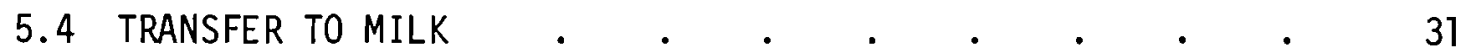

6.0 COMPARISONS WITH OTHER MODELS . $\quad . \quad . \quad . \quad . \quad . \quad . \quad . \quad 33$

6.1 INTRODUCTION

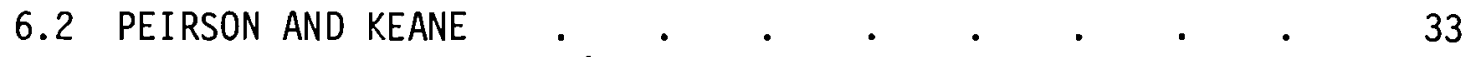

6.3 Pelletier and Voillequé . . . . . . . . . . . . 34

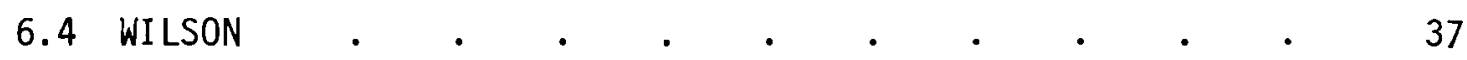

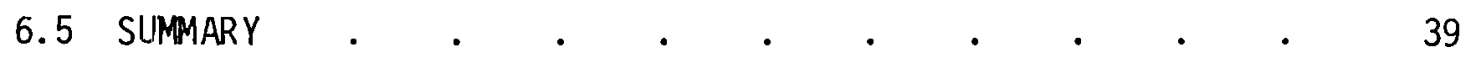

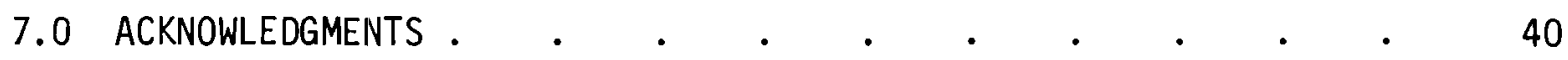

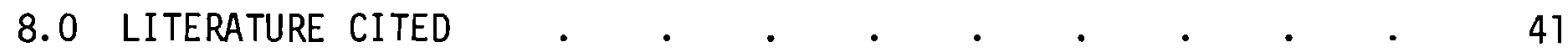

APPENDIX A . . . . . . . . . . . . .

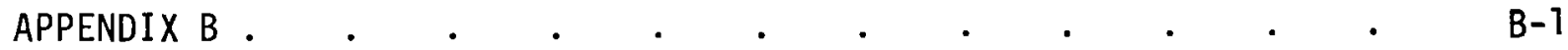




\section{LIST OF FIGURES}

1 Leo Syphus Farm, St. George, Utah . . . . . . . 2

2 Field 3 Looking Southwest from Northeast Corner of

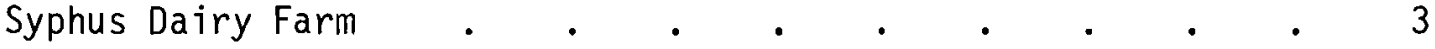

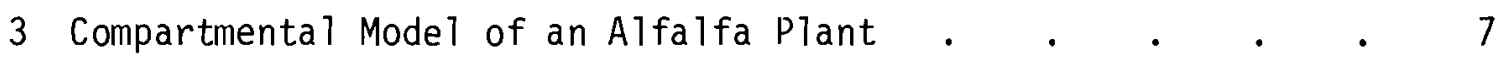

4 Levels of ${ }^{137} \mathrm{Cs}$ on Alfalfa Contrasted with Quantities in Fallout Collectors. $. .5 . \quad . \quad . \quad . \quad 13$

5 Levels of ${ }^{90} \mathrm{Sr}$ on Alfalfa Contrasted with Quantities

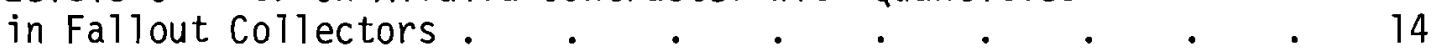

6 Unit Area Levels of ${ }^{137} \mathrm{Cs}$ and ${ }^{90} \mathrm{Sr}$ in/on Alfalfa by Harvest Dates $\left(\mathrm{pCi} / \mathrm{m}^{2}\right) \quad . \quad . \quad . \quad . \quad . \quad . \quad . \quad . \quad 18$

7 Concentration of ${ }^{137} \mathrm{Cs}$ and ${ }^{90} \mathrm{Sr}$ in/on Alfalfa by

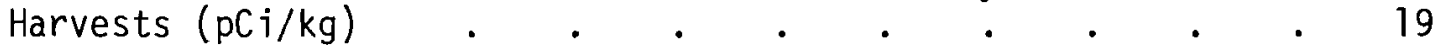

8 Comparison of Estimates of $\lambda$ Calculated from Fallout Collector Data with Intercepts of Linear Regressions [Equation 17] using Alfalfa Data . . . . . . 23

9 Calculated Daily ${ }^{137} \mathrm{Cs}$ from Fallout for Saint George, Utah $\left(\mathrm{pC} i / \mathrm{m}^{2}\right)$ and Ft. Collins, Colorado $\left(\mathrm{pCi} / \mathrm{m}^{3}\right)$. . 26

10 Ratios of ${ }^{137} \mathrm{Cs}$ Levels (Alfalfa/Collectors) Compared to Alfalfa Yields . . . . . . . . . . . . . 29

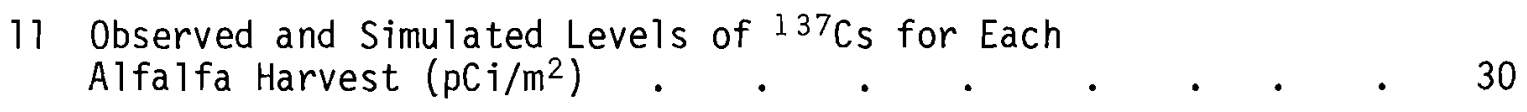

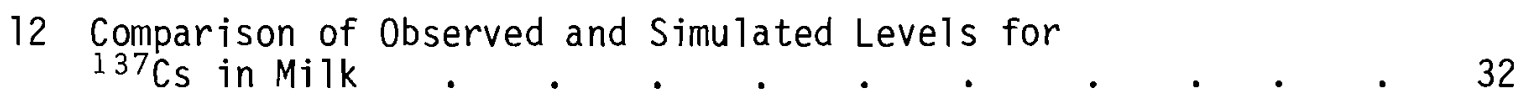




\section{LIST OF TABLES}

1 Concentration of ${ }^{90} \mathrm{Sr}$ and ${ }^{137} \mathrm{Cs}$ in Fallout Collectors (Funnels)

2 Concentration of ${ }^{90} \mathrm{Sr}$ and ${ }^{137} \mathrm{Cs}$ in Fallout Collectors (Pots)

3 Estimates of Daily Rate of Decrease (k) of Fallout . . 10

4 Estimates of Asymptotic Values $(\lambda / k)$ of Fallout Accumulation

5 Alfalfa Harvest Dates, Crop Exposure Intervals, and Radionuclide Concentrations

6 Slope (k) Estimates from Log-linear Regression of Quantities $\left(\mathrm{pCi} / \mathrm{m}^{2}\right)$ of ${ }^{90} \mathrm{Sr}$ and ${ }^{137} \mathrm{Cs}$ in A7falfa Harvests against Time.

7 Concentration $(\mathrm{pCi} / \mathrm{kg})$ of ${ }^{137} \mathrm{Cs}$ and ${ }^{90} \mathrm{Sr}$ in Shoots Grown from Field Root Stocks in Sand Culture. .

8 Ratios of ${ }^{137} \mathrm{Cs}$ Accumulation by Alfalfa to Quantities in Fallout Collectors . . . . . . . 28

9 Differences between Observed and Simulated ${ }^{137} \mathrm{Cs}$ Levels in/on Alfalfa.

10 Estimated Coefficients for ${ }^{140} \mathrm{Ba}$ Deposition on Pasture Grass 


\section{LIST OF TABLES}

IN

APPENDIX A

A1 ${ }^{90} \mathrm{Sr}$ and ${ }^{137} \mathrm{Cs}$ in Individual Funnel Fallout Collectors . . . A.1

A2 ${ }^{90} \mathrm{Sr}$ and ${ }^{137} \mathrm{Cs}$ in Individual Pot Fallout Collectors . . . A.7

A3 Mean Monthly Deviation From the 29 Year Mean of the Daily

Temperature Range at Saint George Power House . . . . . A.12

A4 Mean Monthly Deviation from the 29 Year Mean of the Daily

Precipitation at Saint George Power House . . . . . . A.13

A5 Daily Temperature and Precipitation Record for Saint

George Power House (1963-1966) . . . . . . . . . A.14

A6 Mean ${ }^{137}$ Cesium Concentration per Kilogram and per Square

Meter and Statistics for Each Alfalfa Harvest . . . . . A.26

A7 Mean ${ }^{90}$ Strontium Concentration per Kilogram and per Square

Meter and Statistics for Each Alfalfa Harvest . . . . . A.27

A8 Alfalfa Yield $\left(\mathrm{kg} / \mathrm{m}^{2}\right)$ for Each Harvest . . . . . . . . . A.28

A9 Cesium-137 in Individual Alfalfa Samples . . . . . . . . A.29

A10 Strontium-90 in Individual Alfalfa Samples . . . . . . A.43

Al1 Cs-137/Sr-90 Ratios in Alfalfa Samples . . . . . . . . A.59

A12 ${ }^{90} \mathrm{Sr}(\mathrm{pCi} / \mathrm{kg})$ with Soil Depth in Three Fields (1962) . . . A.71

A13 ${ }^{137} \mathrm{Cs}$ Concentration in Horizontal Composites in Four Fields (1962) . . . . . . . . . . . . . A.72

A14 Comparison of Mean Values ( $\mathrm{pCi} / \mathrm{kg}$ ) of ${ }^{90} \mathrm{Sr}$ and ${ }^{137} \mathrm{Cs}$ for
1962 vs 1965 in Horizontal Composites . $. . \quad . \quad . \quad . \quad . \quad$. A.73

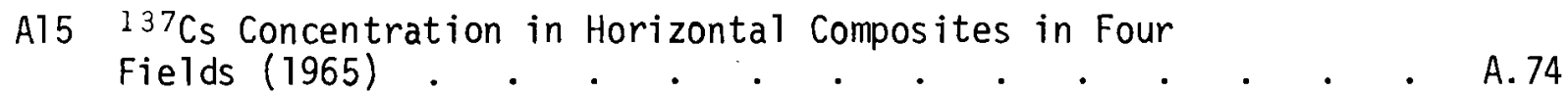


A16 ${ }^{90} \mathrm{Sr}$ Concentrations in Horizontal Soil Composites (1965) . . A.75

A17 Comparison of $137 \mathrm{Cs} /{ }^{90} \mathrm{Sr}$ Ratios in Horizontal Soil

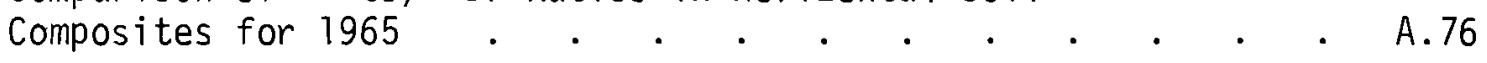

A18 Comparison of ${ }^{137}$ Cs Concentrations in Vertical Soil

Composites for 1965 vs 1962 . . . . . . . . . . A.77

A19 Comparison of $90 \mathrm{Sr}$ Concentrations in Vertical Soil

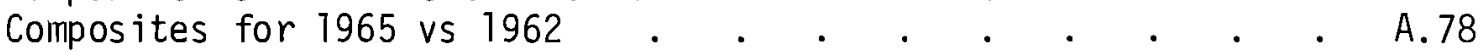

A20 ${ }^{137} \mathrm{Cs}$ and ${ }^{90} \mathrm{Sr}$ Concentration and Ratios in Vertical Soil

Composites (1962) . . . . . . . . . . . . . A.79

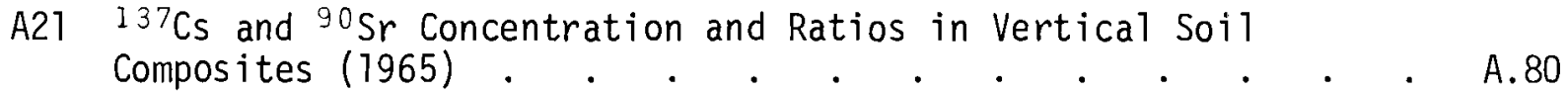

A22 ${ }^{137} \mathrm{Cs}$ and ${ }^{90} \mathrm{Sr}$ Areal Concentration and Ratios in Vertical

Soil Composites (1965). . . . . . . . . . . . A.81

A23 ${ }^{137} \mathrm{Cs}$ and $90 \mathrm{Sr}$ Concentration $(\mathrm{pCi} / \mathrm{l})$ and Solids (mg/l)

in Irrigation Water . . . . . . . . . . . . A.82

A24 Summary of Tests of Linearity for "Square Yard" ( $\left.p C i / m^{2}\right)$

Alfalfa Samples $\left[\log y_{t}=\log \left(C_{1} C_{f} \lambda\right)-k \tau\right]$. . . . A.88

A25 Results of Linear Regressions for "Square Yard" ( $p C i / k g)$

Samples $\left[\log y_{t}=\log \left(C_{1} C_{f}^{\lambda}\right)-k \tau\right]$. . . . . . . A.89

A26 Calculation of Values Used in Figure 8 . . . . . . . . . . A.90

A27 BASIC Computer Program to Make Direct Estimates of

A28 FORTRAN Computer Program Used to Simulate ${ }^{137} \mathrm{Cs}$ Cycling

A29 Sample Output for Surface Air Concentration . . . . . A.131

A30 Estimated Values for Daily Fallout Rate $\left(k_{2}\right)$ • • • • • . A.138

A31 Calculated Daily Fallout Values for ${ }^{137} \mathrm{Cs}$ and ${ }^{90} \mathrm{Sr}$ $\left(\mathrm{pCi} / \mathrm{m}^{2}\right)$ at Saint George, Utah . . . . . . . A.139

A32 Comparison of ${ }^{137}$ Cs Concentrations in "Square Yard",
Field Cut, and Feeding Trial Alfalfa Samples . . . . . A.167 


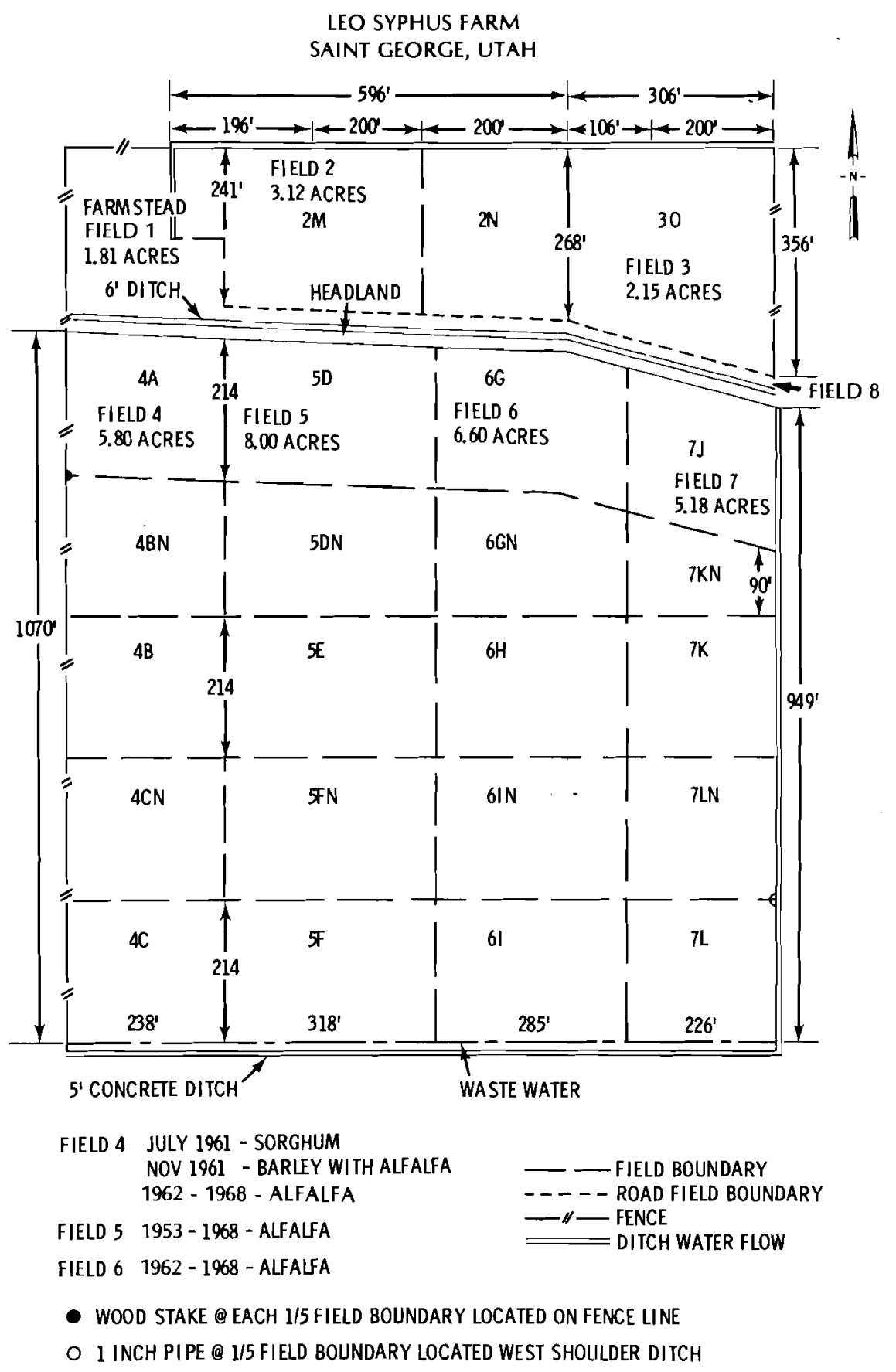

FIGURE 1. Leo Syphus Farm, Saint George, Utah 
monthly collections of fallout through the alfalfa growing season from early April until the fifth cutting in late October. Three pots were exposed during the growth period of barley and ensilage crops. All pots and funnels were exposed from the last alfalfa crop through the winter into April for fallout collection and determination of intercalibration factors.

Steel templates were positioned at randomly-selected coordinates and used to enclose alfalfa shoots $\left(0.91 \mathrm{~m}^{2}\right.$ area) prior to excision by hand clippers (Figure B4). The shoots were cut at "mower" height, immediately enclosed in tared bags to avoid contact with the ground surface and moisture loss, and held out of the sun until "chopped". A large paper cutter was. used to reduce the shoots to approximately $10 \mathrm{~cm}$ lengths which were returned to the labeled sack and frozen with dry ice for transportation to the laboratory. Subsequent processing was identical to that for hay specimens described previously (Hawthorne, 1967). Soil samples were collected with a coring device (Figure B5). Cesium-137 and strontium-90 determinations were by wet-chemistry techniques (Harley, 1962; Wood et a1., 1968).

Many of the mathematical models were fit to observed data by the method of nonlinear least-squares using the "linearization method" (Draper and Smith, 1968) in a program written in BASIC language for the Univac 1108 computer. Several computer simulation models were used in the course of the study and were also implemented on the Univac computer in either FORTRAN or BASIC codes.

\subsection{PREVIOUS MATHEMATICAL MODELS}

The basic formulation for studies of radionuclide uptake and retention is an "input-output" or "compartment" mode1, where the inputs in the present case are radionuclides deposited on alfalfa either from rainfall or as "dry" fallout. The outputs are losses due to washoff in rains, winds and other mechanical actions, loss or removal of plant parts, transport to roots, and physical decay. Previous studies have usually incorporated inputs in terms of radionuclide concentrations in surface air, as well as in rainfall. Thus, Peirson and Keane (1962) show the equation:

$$
\frac{d C_{g}}{d t}+\lambda C_{g}=v_{g} C_{a}+b R C_{r}-k R C_{g}
$$

where:

$$
\begin{aligned}
C_{g} & =\text { quantity of fission product on a unit area of grass } \\
C_{a}, C_{r} & =\text { concentrations per unit volume of air and rain, respectively }
\end{aligned}
$$




$$
\begin{aligned}
& R=\text { rate of rainfall } \\
& \lambda=\text { radioactivity decay rate } \\
& b=\text { fraction retained of current wet deposition } \\
& k=\text { fraction of deposition washed off by unit rainfall }
\end{aligned}
$$

and $v_{g}$ is the "deposition velocity" for dry fallout:

$$
v_{g}=\frac{\text { rate of deposition }\left(\mathrm{pCi} / \mathrm{cm}^{2} / \mathrm{sec}\right)}{\text { concentration in } \operatorname{air}\left(\mathrm{pCi} / \mathrm{cm}^{3}\right)}
$$

They then fit a similar equation with multiple regression methods.

Pelletier and Voillequé (1971) gave their basic equation as:

$$
\frac{d A_{v}}{d t}=a D_{a}+b D_{r}-\left(\lambda+\lambda_{r}\right) A_{v}-R C_{v}
$$

where:

$A_{y}=$ activity on vegetation per unit area of ground

$C_{v}=$ concentration on vegetation (per unit mass)

$D_{a}=$ dry deposition rate per unit area

$D_{r}=$ wet deposition rate per unit area

$\lambda=$ radioactivity decay rate

$\lambda_{r}=$ rate of loss from vegetation

$\mathrm{R}=$ rate of removal of grass by cattle

$a, b=$ interception factors.

Since the above equation incorporates a removal factor (cattle) that operates on mass of vegetation, it is necessary to convert to concentrations to accommodate this removal and the growth of vegetation (assumed to occur at a constant rate), giving:

$$
\frac{d c_{r}}{d t}=\left(4530 k_{r} r+k_{a}\right) c_{a}-\left(\lambda_{e}+\frac{K}{Y}\right) c_{v}
$$

where:

$$
\begin{aligned}
& c_{v}=\text { activity on vegetation per unit mass } \\
& r=\text { rainfall rate } \\
& c_{a}=\text { concentration of radioactivity in surface air }
\end{aligned}
$$


quantities of ${ }^{90} \mathrm{Sr}$ and ${ }^{137} \mathrm{Cs}$ measured in fallout collectors (Figures 4 and 5). Since fallout collectors were not always changed exactly on alfalfa harvest dates, we calculated daily collector accumulations and then integrated these estimates by the procedure outlined under models below. There appears to be a tendency for differences in pattern between harvests, a feature which appears to be associated with harvest yields (cf. Figure 10). Two different dates were used for beginning of fallout exposures for first harvests. The earliest date (about March 20) was selected by interpolation in a table given by Hanson (1972:409), and is generally in accord with a rule-of-thumb developed during the course of growth chamber studies associated with this investigation [growth commences after 4 consecutive days where the average 24-hour temperature, (maximum temperature - minimum temperature)/2 exceeds $\left.55^{\circ} \mathrm{F}\right]$. See Figure B15 where a portion of the weather data at Saint George is presented in this manner. Complete weather documentation for the study period is in Appendix A, Tables 3,4 and 5. The later date ("short" period in Figures 4 and 5; beginning about Apri1 1) coincides with the dates when fallout collectors (funnels) were first changed each spring. Data for the two dates are shown in Figures 4 and 5 to indicate magnitudes of change introduced by use of one or the other. In the remainder of the paper the "long" interval (beginning March 20) is used.

There are two opposing factors affecting the accumulation of fallout on alfalfa plants during the growth of a crop. One is the exponential decline in fallout levels and the other is growth of foliage. Evidently growth provides more surface area so that an individual plant will presumably intercept a larger fraction of fallout as it grows. Expressing the data on a per unit mass basis might tend to compensate for growth, but there are a number of features of growth that could complicate any such interpretation. Moreover, Wilson's (1968) experimental work showed that at least two compartments ("external" and "internal") are needed to adequately represent an alfalfa plant, and the associated losses and transfers may be expected to contribute to overall complexity.

The differential equations implicit in the compartmental model of Figure 3 , assuming constant loss $(\mu, \gamma)$ and transfer $\left(C_{1}, C_{2}\right)$ rates, and an exponentially declining input are:

$$
\begin{aligned}
& \frac{d y_{E}}{d t}=c_{1} \lambda_{i} e^{-k t}-\left(\mu+c_{2}\right) y_{E} \\
& \frac{d y_{I}}{d t}=c_{2} y_{E}-\gamma y_{I}
\end{aligned}
$$

with solutions: 


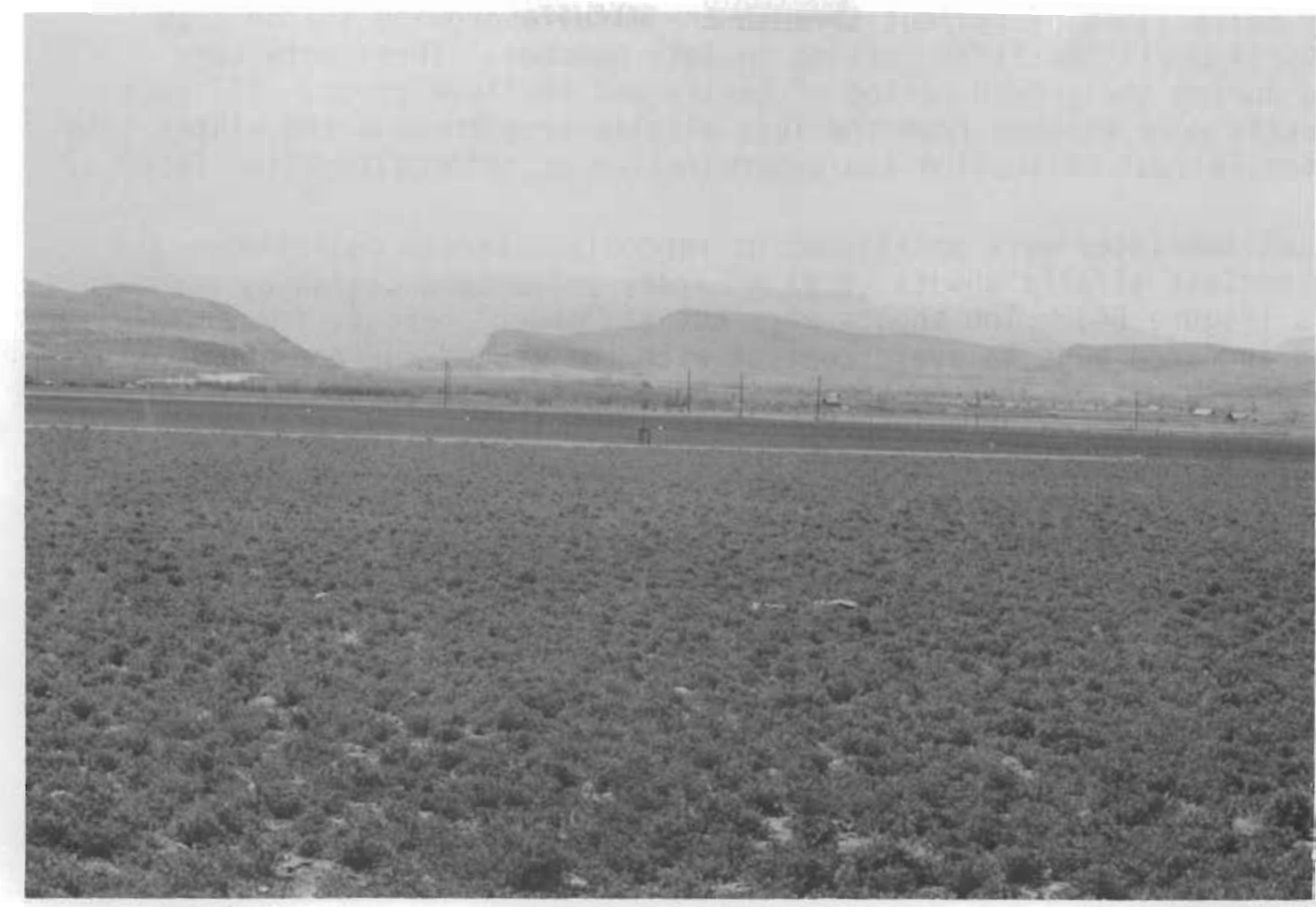

FIGURE 2. Field 3 Looking Southwest From Northeast Corner of Syphus Dairy Farm

\subsection{METHODS}

To assess radionuclide movement and to construct a material(s) balance (an early objective) intensive radionuclide concentration measurements were made on fallout, field grown alfalfa and other crops, soil, irrigation water, and various feed constituents. Ancillary measurements included cow feces and urine samples and measurements on materials for specialized studies. Although stable elements and other radioelement analyses were done, we report only ${ }^{90} \mathrm{Sr}$ and ${ }^{137} \mathrm{Cs}$ results here. Details relative to sampling frequencies and collection periods are in the appropriate section of this report. Weather data were taken from records kept by the Saint George Power House and published in Department of Commerce (Weather Bureau) publications for Utah.

Aerial deposition of radionuclides was sampled in a series of conventional stainless steel pots $\left(0.108 \mathrm{~m}^{2}\right.$, Figure B1) and polyethylene funnels $\left(0.078 \mathrm{~m}^{2}\right.$, Figure B2) mounted above a polyethylene collection flask (9.5 liters) spaced along the boundary between two fields ( 8 and 6.8 acres in size, Figure B3) of the 32.6 acre farm. Funnels were changed at the initiation of each alfalfa harvest. Duplicate steel pots were exposed for 


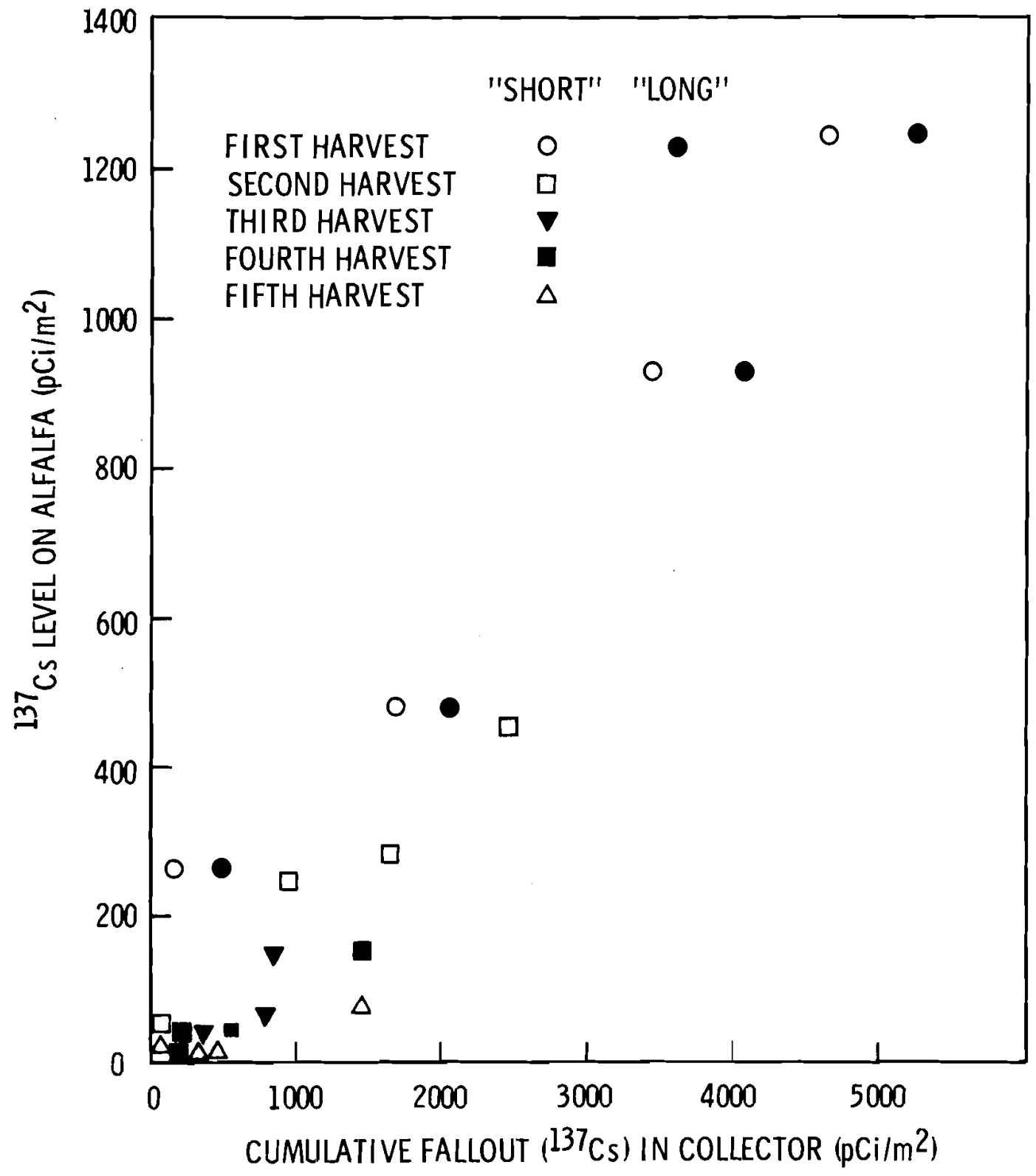

FIGURE 4. Levels of ${ }^{137}$ Cs on Alfalfa Contrasted with Quantities in Fallout Collectors 
were counted. Horizontal soil composites were devised to represent the soil profile in each of several fields and increments were submitted for radioanalysis to assess movement of both radioisotopes into the soil layer. The data available at this writing are presented in Appendix A, Tables A12-A17. In addition, ${ }^{137} \mathrm{Cs}$ and ${ }^{90} \mathrm{Sr}$ concentration values and their ratios (taken from Tables A15-A17) are presented in Figures B16 and B17 for the means of three fields (data from field 80 was not used). The nonlinear, least-squares fits to these data indicate the ${ }^{137} \mathrm{Cs} /{ }^{90} \mathrm{Sr}$ ratios change predictably with depth and that the majority of the change can be associated with ${ }^{137} \mathrm{Cs}$ movement.

The implications of this finding for a deep-rooted plant such as alfalfa are probably confounded by soil-plant factors but should not be ignored in future studies. The data available in Al4 allowed us to calculate the ratio of change for the two years sampled. These calculations indicate that ${ }^{137} \mathrm{Cs}$ increased (1965 vs 1962) in the uppermost and lower levels of the soil profile while ${ }^{90} \mathrm{Sr}(\mathrm{pCi} / \mathrm{kg})$ decreased below 2 inches. Comparable ratios (1965/1962) calculated from vertical composites (A18 and A19) indicate there may be some slight net increase for both radioisotopes in the total soil profile. Ratios ${ }^{137} \mathrm{Cs} /{ }^{90} \mathrm{Sr}$ for vertical soil composites for 1962 and 1965 are shown in Tables A20-A22. Ratios for 1962 averaged $1.48 \pm 0.36$ while the average for 1965 was $1.58 \pm 0.24$. The small sample size for $1962(n=5)$ and the size of the calculated standard deviations preclude any conclusions relative to changes over the 3 -year interval.

Another factor which influences soil radionuclide burden is elements dissolved in irrigation water and/or associated with particulate matter contained therein. Irrigation water used on the farm came from the Virgin River, which drains the Zion National Park area of Southern Utah, thus, flow rates can be influenced by summer thundershowers and spring runoff. For purposes of this study, solids were defined as insoluble material $>0.45 \mu$. Samples were collected (18 1 iters) at a single point in the irrigation ditch above each field, filtered, dried, weighed, and resolubilized for radioisotope determination. Because of limited numbers of samples analyzed and some unexplained high values, we simply present the available data in Table A23. Further analysis will depend on the availability of flow records which at this writing are stil1 in strip chart form.

\subsection{TESTS FOR LINEARITY}

Another way to appraise the data shown in Figure 6 is to test for linearity on the regression lines. The mature alfalfa crops were sampled (at harvest time) by clipping a number of randomly selected areas (square yards, approximately $\left.1 \mathrm{~m}^{2}\right)$. Consequently, these independent determinations of radionuclide burden can be used in a test of linearity (Draper and Smith, 1966). These tests showed highly significant deviations from linearity in a 11 cases for the $\mathrm{pCi} / \mathrm{m}^{2}$ data (details are in $\mathrm{A} 24$ and 
regression calculations using $\mathrm{pCi} / \mathrm{kg}$ are in $\mathrm{A25}$ ). The results may be both an indication that the regression model [equation (17)] is only a first-order approximation to the complex underlying model [equation (11)] and a consequence of factors not accounted for by the model (eg. remobilization of radionuclides from root stocks, soil chemistry or irrigation water). The fact that the regression model provides estimates of the parameter $k$ in accord with independent estimates (Tables 3 and 6 ) lends support to its utility as an approximation.

A further appraisal of the utility of the regression lines of Figure 6 can be accomplished by noting that the intercepts $\left[\log \left(C_{l} C_{f} \lambda\right)\right.$ in equation (17)] should vary from year to year in proportion to the current "spring peak" level of fallout $(\lambda)$. The spring peak values $(\lambda)$ have been independently estimated from fallout-collector data (see Appendix A26 for details), and Figure 8 shows the relationship between the two quantities. The slope of the line in Figure $8(20.6)$ then estimates $C_{1} C_{f}$, a quantity which we assume to be constant throughout. The generally good fit to a straight line in Figure 8 supports that assumption and its foundation in the assumed suitability of equation (17) as an approximation to the overall model of equation (11).

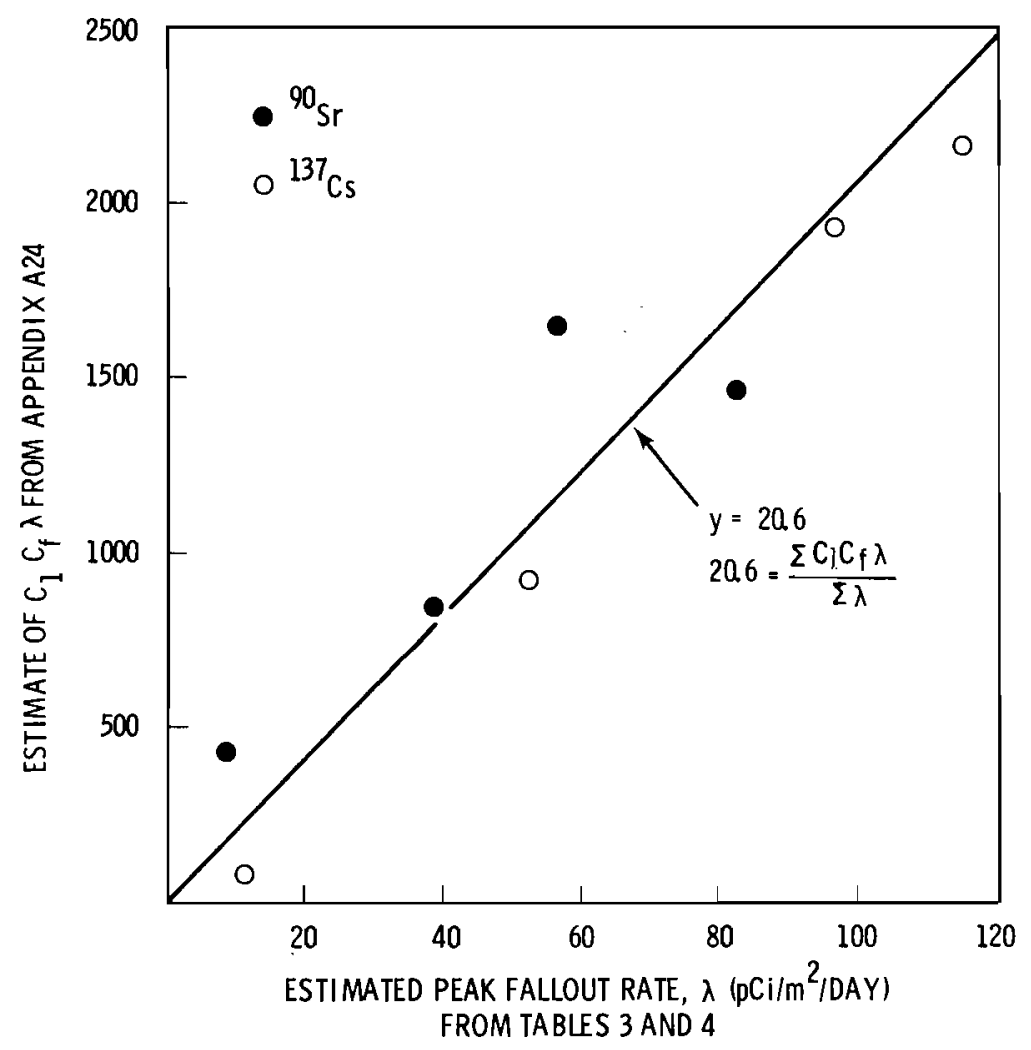

FIGURE 8. Comparison of Estimates of $\lambda$ Calculated from Fallout Collector Data with Intercepts of Linear Regressions [Equation 17] using Alfalfa Data 


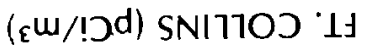

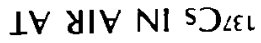

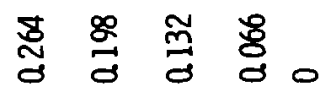

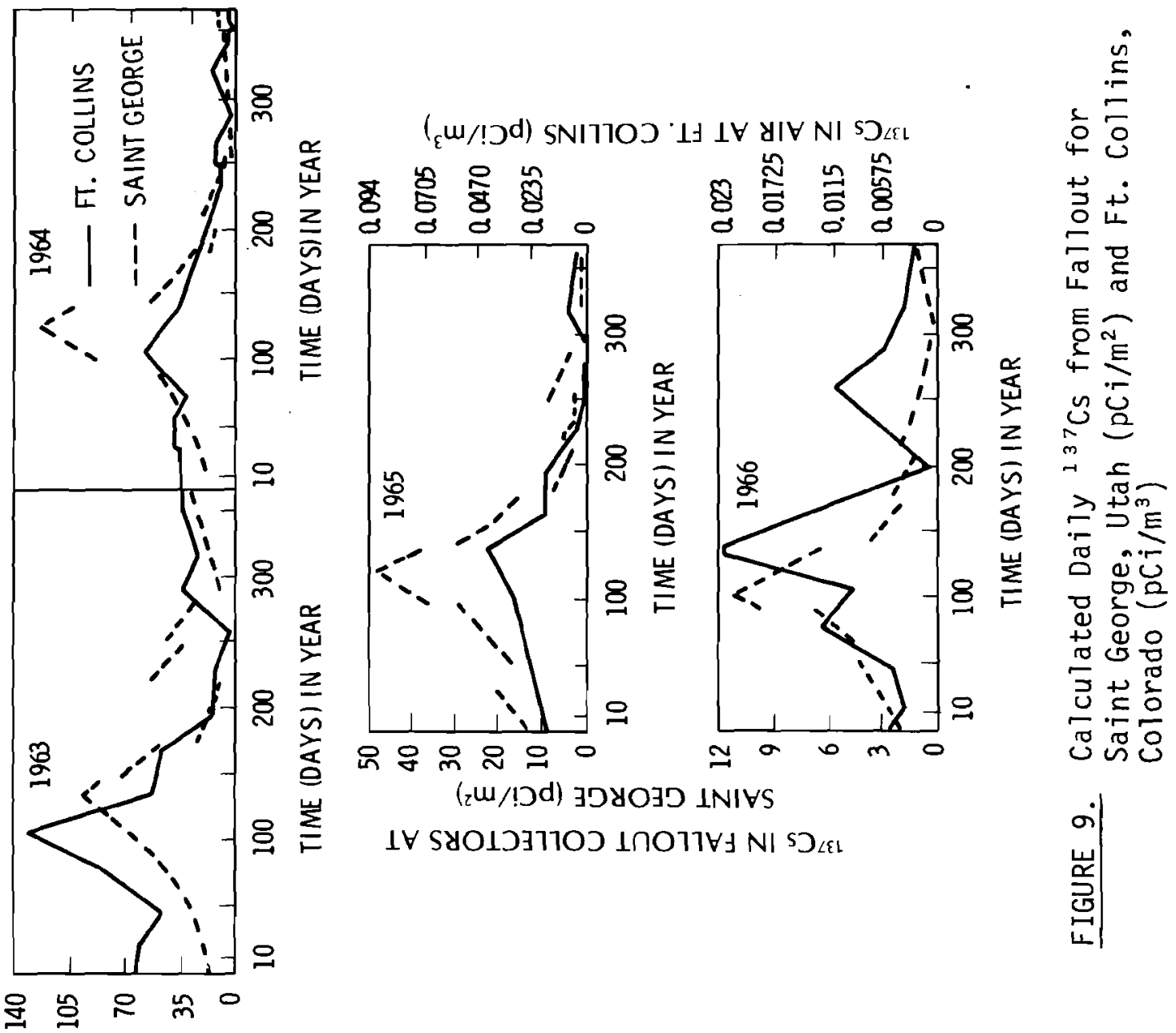

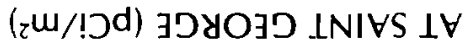

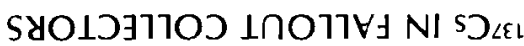


The above procedure necessarily fails for those periods containing the "spring peak" of fallout. To derive estimates for these periods, we estimated the actual date of the spring peak from a joint solution of the equations representing the preceding ("increase phase") and succeeding ("decrease phase") fallout collections (B19). The collection judged to include the spring peak was then divided into sections corresponding to increasing and decreasing fallout on the basis of the estimated peak fallout date, total in the collector, and the two values of $k$ given above (B20). The complete calculation is illustrated in Appendix B21 and the daily numerical results for both ${ }^{137} \mathrm{Cs}$ and ${ }^{90} \mathrm{Sr}$ are in A31. Collector data used; data files TEMP and SRFALL, respectively, and the computer subroutine (TFALL2) are in $A 28$.

There are various other procedures that might have been utilized to derive fallout inputs to the simulation model. The method we chose was largely based on our feeling that the identity of the individual fallout collections should be preserved insofar as possible, as a way of examining overal1 consistency of the data. An inspection of Figure 9 will show that the input data do in fact seem to follow a consistent pattern and that the pattern of the collectors is in accord with the Colorado air data (Wilson, 1968). Exceptions are the last two "decrease phase" periods of 1963 and the peak period in 1964. Considerably more detail of the procedure and the algebra is in Thomas et al., 1975b.

\subsection{ALFALFA PLANTS}

The next stage of the model involves deposition of fallout on alfalfa plant surfaces [Wilson's (1968) "exterior" compartment]. The major difficulty here has to do with the problem of devising a satisfactory model for the interception of fallout by plants, including the related matter of determining how interception changes as plants grow. So far as we know, none of the many previous studies of fallout on plants have satisfactorily resolved these difficulties, so we have simply introduced enipirical corrections, as described below. Since a comparison of fits of equation (17) to concentration of fallout on alfalfa plants seemed more satisfactory when concentration was expressed on the basis of $\mathrm{pCi} / \mathrm{m}^{2}$ (as in Figure 6), we have elected to use that representation in the simulation (rather than $\mathrm{pCi} / \mathrm{kg}$, Figure 7).

A comparison of fallout accumulation by alfalfa with the quantities estimated to be in collectors (calculated by summing appropriate quantities in A31) during the same time intervals (Table 8 ) shows a pronounced decrease among succeeding harvests, within years.

If these ratios are compared to alfalfa yields (Figure 10), there is definite evidence of higher fallout interception at the larger yields. 
TABLE 8. Ratios of ${ }^{137} \mathrm{Cs}$ Accurmulation by Alfalfa to Quantities in Fallout Collectors

\begin{tabular}{lccccc} 
& \multicolumn{7}{c}{ Harvest } \\
\cline { 2 - 6 } Year & $\underline{1}$ & $\frac{2}{1}$ & $\frac{3}{4}$ & $\underline{4}$ & $\underline{5}$ \\
1963 & 0.228 & 0.183 & 0.187 & 0.107 & 0.052 \\
1964 & 0.239 & 0.171 & 0.091 & 0.097 & 0.189 \\
1965 & 0.237 & 0.260 & 0.116 & 0.154 & 0.066 \\
1966 & 0.582 & 0.269 & -- & 0.485 & 0.473
\end{tabular}

Consequently, we have introduced simple weighting factors into the model based on the average yield for each harvest (averaged over the 4 years, see Appendix A8). These factors were calculated by assigning a weight of unity in the first harvest, and reducing fallout collection efficiency in succeeding harvests by a fraction calculated as the mean yield in that harvest divided by that for the first harvest. The resulting coefficients are 1.00, $0.678,0.658,0.550$, and 0.366 for the five harvests. Milbourn and Taylor (1965) and ward et al., (1965) have shown a similar relationship between retention and crop yield. In effect, these coefficients serve to replace the growth model of equation (12).

We calculated an "interception factor" or "transfer coefficient" (from daily fallout quantities to deposition on alfalfa plants) by running the simulation model with that coefficient set at unity (1ine 108, main program, A28) and then calculating the coefficient ( $C_{1}$ of Figure 3$)$ as:

$$
\text { transfer coefficient }=\frac{\sum \text { observed }{ }^{137} \mathrm{Cs} \text { concentration on alfalfa }}{\sum \text { simulated }{ }^{137} \mathrm{Cs} \text { concentration on alfalfa }}
$$

The value thus obtained (0.488) applies directly to only the first alfalfa harvest of each year. The effective interception coefficients for the remaining harvests must be adjusted by the factors listed in the paragraph just above. Thus the sequence of fractions intercepted by successive harvests become $0.488,0.330,0.321,0.268,0.178$ (1 ine 110, main program, A28).

The exterior compartment loses radionuclides in two ways--by transfer to the interior, and by losses directly from the surface (through wind action, rain, and other agencies). This latter factor has been assessed in a sizable number of studies, and the consensus seems to be that a rate of about 0.05 of the quantity present is lost per day (or an effective halftime of about 14 days). While we have adopted that value for the present 


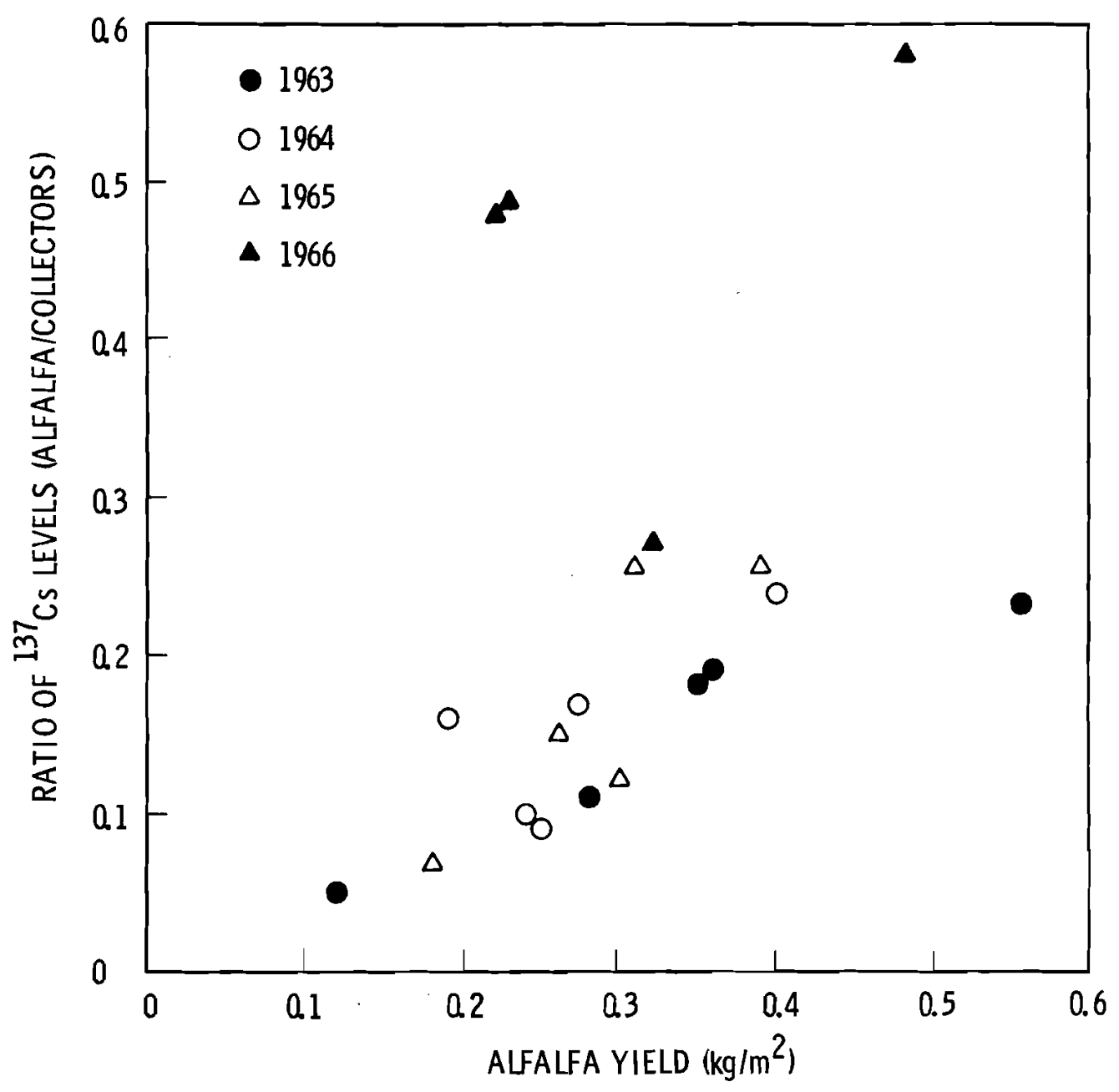

FIGURE 10. Ratios of ${ }^{137} \mathrm{Cs}$ Levels (Alfaifa/Collectors) Compared to Alfalfa Yields 
Inasmuch as the results of 27 different feeding trials, expressed as a simple transfer coefficient, were quite consistent (Hawthorne, 1966), we have simply used the mean value of these transfer coefficients as an equilibrium value for the simulated "cow", in place of the rather complex model required if dietary inputs are permitted to fluctuate ( $C f$. Pelletier and Voilleque, 1971). A factor complicating our analysis of the data is the fact that it was necessary, on three occasions in 1964, to feed hay from another location (Enterprise, Utah). These data sets are excluded from Figure 12, which shows observed and simulated milk levels for the remaining data sets (the excluded data sets are included in Appendix A29). With the exception of the last harvest of 1963 and the first harvest of 1964, it appears that the observed and simulated data are generally in satisfactory agreement.

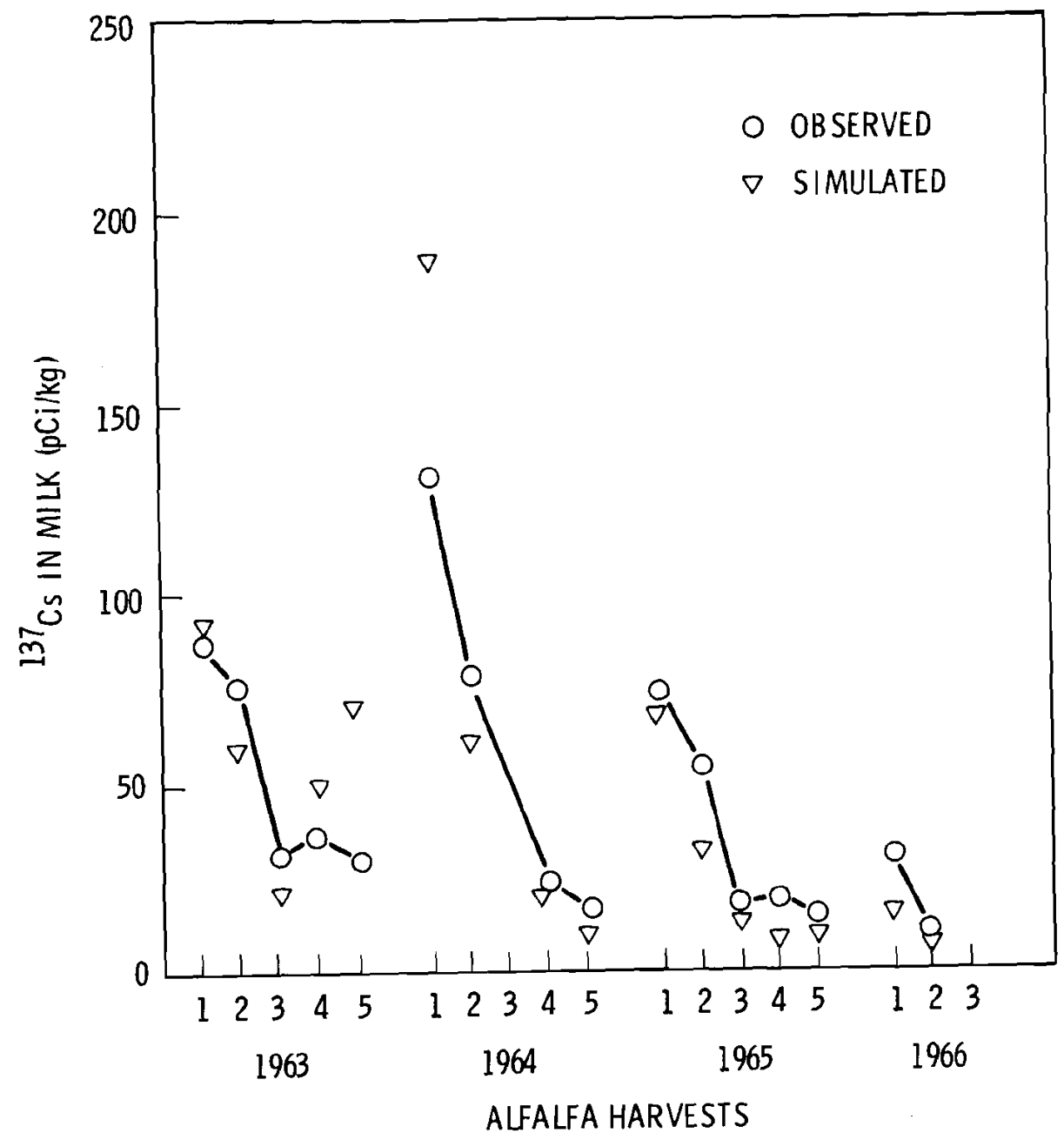

FIGURE 12. Comparison of Observed and Simulated Levels for ${ }^{37} \mathrm{Cs}$ in Mi $7 \mathrm{k}$ 


\subsection{COMPARISONS WITH OTHER MODELS}

\subsection{INTRODUCTION}

Ideally, a model should be "validated" by testing on data that has not been used in its development. One way to attempt such a test is to partition the original data into two sets, one to be used in model construction, the other for validation. This does not seem to be a reasonable approach in the present case, in consequence of the low levels of fallout in the last full year of the study and the evidence of a significant contribution of ${ }^{137} \mathrm{Cs}$ burden in foliage by transfer from root stocks. A more compelling argument against such tests over time is that those biases associated with the derivation of parameters from part of a set of data are likely to remain constant throughout the entire set and may go undetected. Therefore, comparisons with other, similar models are needed for an appraisal of our results. Various comparisons with independent estimates of the parameters have already been presented in development of the model, so we deal here with certain broader comparisons having to do with model structure.

\subsection{PEIRSON AND KEANE}

Peirson and Keane (1962) derived their parameter estimates from the coefficients of a multiple regression model based on a differential equation [equation (1) above] and fit to observed data. Since their observations mainly concerned barium-140 and iodine-131 on "pasture grass, "we cannot expect to make exact comparisons. For $140 \mathrm{Ba}$, the main parameters and standard errors were estimated by Peirson and Keane (1962) as:

$$
\begin{aligned}
& \text { dry deposition velocity }(\mathrm{cm} / \mathrm{sec})=v_{g}=0.26 \pm 0.24 \\
& \text { fractional retention of rain-deposited radioactivity }=b=1.00 \\
& \pm 0.3 \\
& \text { fractional removal of radioactivity per unit rainfall }(\mathrm{mm})=k \\
& =0.009 \pm 0.018
\end{aligned}
$$

The magnitude of the standard errors associated with the parameters prohibits any exactness in comparisons. In any case, we are mainly concerned here with the question of whether or not parameters having to do with retention and washoff, both resulting from the same events, i.e., rainfall, might reliably be estimated from observed data alone. Before actually fitting the data, Peirson and Keane arbitrarily introduced a change of variable, $b=1-m R$ ( $R$ is ratio of rainfall and $m$ an unknown constant), that does not seem necessary if the model is in fact the appropriate realization of actual phenomena. We, therefore, fit equation (1) directly to the data [Table 3 of Peirson and Keane (1962)]. Since Peirson and Keane indicate (Fig. 2 of their paper) that $\mathrm{vg}$ decreases markedly during the course 
of the observational period, we also studied a modified form of their equation (1) by introducing the reciprocal of time (weeks) into the term containing $\mathrm{vg}_{\mathrm{g}}$. Fits to the two equations yielded the coefficients given in Table 10.

TABLE 10. Estimated Coefficients for ${ }^{140}$ Ba Deposition on Pasture Grass

\begin{tabular}{lllll} 
& $\begin{array}{c}\text { Dry deposition } \\
v_{g}(\mathrm{~cm} / \mathrm{sec})\end{array}$ & $\begin{array}{c}\text { Retention } \\
b\end{array}$ & & $\begin{array}{c}\text { Wash-off } \\
k\end{array}$ \\
\cline { 2 - 3 } $\begin{array}{l}\text { Direct fit to equation (1) } \\
\text { of Peirson and Keane }\end{array}$ & $0.37 \pm 0.46$ & & $0.91 \pm 0.32$ & $0.03 \pm 0.24$ \\
Term in $v_{g}$ modified & $0.26 \pm 0.79$ & & $0.83 \pm 0.32$ & $-0.10 \pm 0.15$ \\
Results of Peirson and Keane & $0.26 \pm 0.24$ & & $1.00 \pm 0.30$ & $0.009 \pm 0.018$
\end{tabular}

The results of chief interest here are that the parameters $v_{g}$ and $b$ remain fairly stable in the several versions, but the wash-off factor $k$ varies considerably, even changing sign (a result inconsistent with definition of the factor). We note, however, that these results may be entirely spurious inasmuch as only the coefficient $b$ turns out to be statistically significant in the regression analysis for the two models we considered. Another shortcoming of the model is that it does not include a term for losses that occur independently of rainfall, a factor shown by later investigators to be of considerable importance.

Since we do not have records of air concentration at Saint George, we are able to make a direct comparison only with the retention factor, $b$, which in the Saint George data ranges from 0.49 (first harvest) down to 0.19 in the fifth harvest, one-fourth to one-half the value observed by Peirson and Keane (ibid.).

\subsection{Pelletier AND VOILLeQÚ́}

Pelletier and Voillequé (1971) start with an equation similar to that of Peirson and Keane, apart from addition of a term to represent removals by cattle, but convert the equation to represent concentration per unit mass $(\mathrm{pCi} / \mathrm{kg})$ before fitting it to data. They use only air concentration data, introducing "washout" by rain in terms of an equation using an overall deposition rate which they define as:

$$
D=C_{a}\left(V_{d}+g \cdot r\right)
$$


where:

$$
\begin{aligned}
& D=\text { deposition rate on collectors }\left(\mathrm{pCi} / \mathrm{m}^{2} / \text { day }\right) \\
& C_{a}=\text { average air concentration }\left(\mathrm{pCi} / \mathrm{m}^{3}\right) \\
& V_{d}=\text { empirical constant for dry deposition }(\mathrm{m} / \text { day }) \\
& g=\text { empirical constant for washout by } \mathrm{rain}(\mathrm{m} / \mathrm{cm} \mathrm{rain}) \\
& r=\text { rainfall rate }(\mathrm{cm} \text { rain/day) }
\end{aligned}
$$

Using results of a previous study (Pelletier et al., 1965), Pelletier and Voilleque convert the above equation to

$$
D=C_{a}(200+4530 r)
$$

For comparison to Saint George data, we note that the ratio $D / C_{a}$ corresponds to the deposition velocity constant as usualiy defined (rate of deposition/ concentration in air) so we can calculate it as:

$$
V_{g}=\frac{D}{C_{a}}=200+4530 r
$$

Using the mean annual rainfall for 1963-1966 at Saint George (7.4 inches, A4), we calculate a value of $V_{g}$ as $433 \mathrm{~m} /$ day, using Pelletier and Voillequé's equation. The only comparison available to us is a value of $\mathrm{V}_{\mathrm{g}}$ calculated by using the Saint George fallout collector data and the Colorado (Ft. Collins) air concentrations shown in Figure 9. The overall ratio of these two sets of data is $530 \mathrm{~m} /$ day. It is of interest here that Wilson (1968) shows a general agreement between the Ft. Collins air data and observations made at Chicago. A graphical comparison with the 1964 and 1965 air concentration data given by Pelletier and Whipple (1968) shows very much the same pattern as the Chicago data. These comparisons do not, of course, establish any correspondence between air concentration at the other sites and those that existed at Saint George. We note, however, that Peirson and Keane (1962) found dry deposition velocities $(140 \mathrm{Ba})$ of $0.5 \mathrm{~cm} / \mathrm{sec}(430 \mathrm{~m} /$ day) to an artificial grass surface in England, while Wilson, Ward, and Johnson (1967) cite some Norwegian data yielding $500 \mathrm{~m} /$ day for fallout beta activity in collectors.

Pelletier and Voillequé (1971) use equation (3) as their model for fitting observed radionuclide data on pasture grass. The loss rate from vegetation $\left(\lambda_{e}\right)$ is that generally reported (14 day half-time) and used in the Saint George study. The factor $K$ is a (1inear) growth rate for grass, for which values are not given by Pelletier and Voillequé. The essential part of their model for comparison to Saint George data is that concerning transfer from air to grass:

$$
D_{v}=\left(k_{a}+4530 \cdot k_{r} \cdot r\right) c_{a}
$$


where $D_{y}$ is ${ }^{137} \mathrm{Cs}$ concentration $(\mathrm{pCi} / \mathrm{kg}$ ) on grass, $r$ is rainfal1 rate $(\mathrm{cm} /$ day $), 4530$ is the empirical constant described above in conjunction with equation (20), $k_{a}$ represents "deposition velocity per unit areal grass density $\left(\mathrm{m}^{3} / \mathrm{kg} /\right.$ day $)$," and $\mathrm{kr}$ is the "fraction of the activity intercepted and retained per unit grass density $\left(\mathrm{m}^{2} / \mathrm{kg}\right)$." Values for $\mathrm{k}_{r}$ and $\mathrm{k}_{\mathrm{g}}$ are obtained by a trial and error process that seeks to minimize the normalized absolute mean difference $(\Delta)$ between observed and simulated data while also selecting that set of parameters that minimizes the standard deviation of the calculated values of $\Delta$. The technique thus is regression-like in its properties (apart from a complication introduced by the attempt to "normalize" around zero, the technique corresponds directly to the method of least squares and thus to regression calculations). There is thus, as with Peirson and Keane's (1962) study, the question of whether regression methods can be depended on to accurately estimate deposition and retention coefficients from observed data.

Pelletier and Voillequé estimated $\mathrm{kr}$ for ${ }^{137} \mathrm{Cs}$ as $1.2 \mathrm{~m}^{2} / \mathrm{kg}$ (Table 2 of their paper), and report average grass density to be $0.062 \mathrm{~kg} / \mathrm{m}^{2}$, from which we estimate retention per square meter to be $1.2(0.062)=0.074$, which suggests a rather low interception efficiency in terms of the various experiments in which radionuclides have been artificially sprayed on pasture plants (Milbourn and Taylor, 1965; Middleton, 1960). Combining the various coefficients of equation (23) yields an overall predictive relationship for $13{ }^{7} \mathrm{CS}$ of:

$$
D_{V}=[950+4530(1.2) 0.25]=2309 C_{a}
$$

(using the average rainfall of $0.25 \mathrm{~cm} /$ day in the 24 week study of Pelletier and Voilleque). Comparison with the Saint George data is again hampered by our lack of air concentration data. We can, however, use Pelletier and Voilleque's conversion equation [equation (21) above] to translate to an equivalent fallout rate to collectors, giving:

$$
D=C_{a}[200+4530(0.25)]=1330 c_{a}\left(p C i / m^{2} / \text { day }\right)
$$

Changing $D_{y}$ above to a per unit area basis is accomplished by using their average grass density of $0.062 \mathrm{~kg} / \mathrm{m}^{2}$, giving:

fallout on grass per unit area $=D_{f}=0.062\left(\mathrm{~kg} / \mathrm{m}^{2}\right) \cdot D_{v}(\mathrm{pCi} / \mathrm{kg})$

$$
D_{f}=0.062(2309) C_{a}=143 C_{a}
$$

and,

$$
\text { total interception efficiency }=\frac{D_{f}}{D}=\frac{143}{1330}=0.11
$$


It may be noted that this is a somewhat lower value than our calculation of an efficiency of 0.18 for fifth harvest alfalfa mentioned in Section 5.3. However, Figure 10 shows a generally good correlation between interception and yield (excepting the 1966 data, which we believe was affected by remobilization of ${ }^{137}$ Cs from root stocks), and also shows virtually all of the St. George yields to be in excess of $0.20 \mathrm{~kg} / \mathrm{m}^{2}$ as contrasted to $0.06 \mathrm{~kg} / \mathrm{m}^{2}$ in the Michigan study.

\subsection{WILSON}

In the models discussed thus far, there are questions as to how well regression or regression-like techniques can be used to jointly estimate the relative contributions of dry and wet deposition of fallout radionuclides to burdens observed on plants. In the Colorado studies (Wilson, 1968; Wilson et a1., 1967) direct experimental evidence was obtained (see particularly Wilson et al., 1967) indicating that contamination levels of ${ }^{137} \mathrm{Cs}$ on alfalfa were not dependent on rainfall, and suggesting dry fallout to be the primary contaminating source. In the absence of air concentration data for Saint George, we have necessarily been constrained to use transfer coefficients based on fallout accumulation in collectors. Our comparisons with the other studies thus must depend on conversion of the published results to correspond to the Saint George data.

The predictive model obtained in the Colorado study depends essentially on a "transfer rate" obtained by regression analysis. The relationship is given by Wilson et al., (1967, Figure 2, p. 128) and Wilson (1968, p. 108) as:

$$
A_{n}=a_{0} k_{n} v_{\max }+S
$$

where:

$$
\begin{aligned}
A_{n} & =\text { foliar contamination level }\left(\mathrm{pCi} / \mathrm{m}^{2}\right) \\
a_{0} & =\text { air concentration level as crop begins growth } \\
k_{n} & =\text { plant contamination factor (expressed in days) } \\
S & =\text { contribution from soil to foliar contamination } \\
V_{\max } & =\text { upper limit to deposition velocity }
\end{aligned}
$$

The plant contamination factor, $k_{n}$, represents the accumulated burden at the nth day in a two-compartment model representing an alfalfa plant ( $\mathrm{C} f$. Figure 13 of Wilson, 1968). Wilson's procedure was to regress observed alfalfa contamination levels $\left(A_{n}\right)$ on the products of $a_{0}$ (separate values used for each of the alfalfa harvests) and $k_{n}$ to obtain estimates of $V_{\max }$ (990) and $S(-60)$. Since $S$ turned out to have a negative coefficient (not 


\subsection{ACKNOWLEDGMENTS}

The results of this study depend on the long-term contribution of many individuals. Special recognition is extended to L. T. Syphus and E. E. Syphus of Saint George, Utah, who allowed their dairy operation to be used as the site of the study, and cooperated in many other ways. Milk production records were kindly supplied by Wayne and Grant Whitehead of the Saint George Ice Company. Precise technical assistance was provided by E. B. Douglas, D. F. Humphrey, W. J. Siembab, and T. Hartsock under conditions that were at times quite uncomfortable. R. A. Wood consistently provided accurate low-level radiochemical analyses for a variety of samples and was remarkably patient with the demands occasioned by our field and laboratory procedures. F. M. Plesset wrote the original set of computer data reduction programs. Ms. Marji I. Cochran provided data reduction and computer programing expertise during the analysis phase of the study and $R$. L. Wildung critically read the portions dealing with soil science. Administrative support and encouragment from W. A. Rhoads and J. F. Ross are gratefully acknowledged. 


\subsection{LITERATURE CITED}

Ambler, J. E. and R. C. Menze1. 1966. Retention of foliar applications of $85 \mathrm{Sr}$ by several plant species as affected by temperature and relative humidity of the air. Radiation Botany $6: 219$.

Draper, N. R. and H. Smith. 1966. Applied Regression Analysis. John Wiley and Sons, New York.

Hanson, C. H. (ed.) 1972. Alfalfa Science and Technology. Volume 15 in the series AGRONOMY, American Society of Agronomy, Madison, Wis.

Harley, J. H. (Dir.) 1962. Manual of Standard Procedures, Health and Safety Laboratory, U.S. Atomic Energy Commission Report, NY0-4700 (Rev.), New York.

Hawthorne, H. A. 1965. Fission-product cycles in an agricultural system. 1. Sample heterogeneity. pp. 711-722. In: Radioactive Fallout from Nuclear Weapons Tests (A. W. Klement, Jr., ed.).

Hawthorne, H. A. 1967. Field studies of the transfer of ${ }^{137} \mathrm{Cs}$ from fallout to milk, pp. 77-85. In: Radioecological Concentration Processes (B. Aberg and F. P. Hungate, eds.), Pergamon Press, Oxford.

Hawthorne, H. A., S. D. Zellemer, L. L. Eberhardt, and J. M. Thomas. 1975. ${ }^{137}$ Cs cycling in a Utah dairy farm. Submitted to Health Physics.

Johnson, J. E., D. W. Wilson, and W. L. Lindsay. 1966. Transfer of fallout cesium-137 from soil to dairy cattle feeds. Soil Sci. Soc. Amer. Proc. 30:416.

Kirchmann, R., E. Fagniart, and S. Van Puymbroeck. 1967. Studies on foliar contamination by radiocesium and radiostrontium. pp. 475-483. In: Radiological Concentration Processes (B. Aberg and F. P. Hungate, eds:), Pergamon Press, Oxford.

Middleton, L. J. 1960. Radioisotopes in plants: Practical aspects of aerial contamination with strontium-89 and cesium-137. In: Radioisotopes in the Biosphere (R. S. Caldecott and L. A. Snyder, eds.), University of Minnesota, Minneapolis.

Milbourn, G. M. and R. Taylor. 1965. The contamination of grassland with radioactive strontium. I. Initial retention and loss. Radiation Botany $\underline{5}: 337$.

Moorby, J. and H. M. Squire. 1963. The loss of radioactive isotopes from the leaves of plants in dry conditions. Radiation Botany $\underline{3}: 163$. 
Nishita, H. and H. A. Hawthorne. 1967. Effect of moisture tension on the concentration of tracer Sr ${ }^{85}$ in expressed soil solution. Soil Sci. 103(5): 339 .

Peirson, D. H. and J. R. Keane. 1962. Characteristics of early fallout from the Russian nuclear explosions of 1961. Nature 196:801.

Pelletier, C. A., G. H. Whipple and H. L. Wedlick. 1965. Use of surfaceair concentration and rainfall measurements to predict deposition of fallout radionuclides. pp. 723-736. In: Radioactive Fallout from Nuclear Weapons Tests (A. W. Klement, Jr., ed.) CONF-765.

Pelletier, C. A. and G. H. Whipple. 1968. Investigations in to the Cycling of Certain Radionuclides through the Milk-Food Chain. Final Report No. CO0-5000-1, University of Michigan, Ann Arbor.

Pelletier, C. A. and P. G. Voillequé. 1971. The behavior of ${ }^{137} \mathrm{Cs}$ and other fallout radionuclides on a Michigan dairy farm, Health Phys. 21:777.

Romney, E. M., J. W. Neel, H. Nishita, J. H. 01 afson, and K. H. Larson. 1957. Plant uptake of $\mathrm{Sr}^{90}, \mathrm{Y}^{91}, \mathrm{Ru}^{106}, \mathrm{Cs}^{137}$, and $\mathrm{Ce}^{144}$ from soils, Soil Sci. 83(5): 369 .

Russe11, R. S. 1965. An introductory review. Interception and retention of airborne material on plants. Health Phys. 11:1305.

Thomas, C. W., J. A. Young, N. A. Wogman, and R. W. Perkins. 1970. The measurement and behavior of airborne radionuclides since 1962. In: Radionuclides in the Environment. Volume 93 in the Series Advances in

Chemistry, American Chemical Society, Washington, D.C.

Thomas, J. M., L. L. Eberhardt, H. A. Hawthorne, and S. D. Ze11mer. 1975a. Cesium-strontium ratios in an intensive study of fallout. In: Radioecological Problems Associated with the Development of Energy Sources. Fourth National Symposium on Radioecology. Dowden, Hutchinson and Ross, Inc., Stroudsburg, PA. (In press).

Thomas, J. M., L. L. Eberhardt, H. A. Hawthorne, and S. D. Ze11mer. $1975 b$. Reconstruction of fallout patterns from collector data. In: Radioecological Problems Associated with the Development of Energy Sources. Fourth National Symposium on Radioecology. Dowden, Hutchinson and Ross, Inc., Stroudsburg, PA. (In press). 
Thompson, S. E. 1965. Effective Half-life of Fallout Radionuclides on Plants with Special Emphas is on Iodine-137. UCRL-12388, UC-41, TID-4500, University of California, Lawrence Radiation Laboratory, Livermore, Cal ifornia.

Ward, G. M., J. E. Johnson, and H. F. Stewart. 1965. Cesium-137 passage from precipitation to milk. In: Radioactive Fallout from Nuclear Weapons Tests. pp. 703-710. (A. W. Klement, Jr., ed.).

Wilson, D. W., G. M. Ward, and J. E. Johnson. 1967. Fallout 137 Cs: Direct aerial transfer as an important source of foliar deposition. Radiation Botany 7:313-319.

Wilson, D. W. 1968. A Mathematical Model of the Transport of Cesium-137 from Fallout to Milk. Ph.D. Thes is, Colorado State University, Ft. Collins, Colorado.

Wilson, D. W., G. M. Ward, and J. E. Johnson. 1969. A quantitative model of the transport of ${ }^{137} \mathrm{Cs}$ from fallout to milk. In: Agriculture and Public Health Aspects of Environmental Contamination by Radioactive Materials, pp. 125-134, International Atomic Energy Agency, Vienna.

Wood, R. A., S. T. Wakakuwa, T. H. Rutherford, A. L. Beck, and R. V. Roscoe. 1968. The Sequential Separation and Quantitative Determination of $5 r^{90}$, $\mathrm{Ce}^{144}, \mathrm{Cs}^{137}$ and the Stable Elements of $\mathrm{Sr}$ and $\mathrm{Ca}$ in Plant, Feces, and Water Samples. UCLA 12-676, UC-48 Biology and Medicine, TID-4500, Laboratory of Nuclear Medicine and Radiation Biology, 900 Veteran Avenue, Los Angeles, Cal ifornia. 
APPENDIX A 
APPENDIX A

TABLE Al. $\quad{ }^{90} \mathrm{Sr}$ and ${ }^{137} \mathrm{Cs}$ in Individual Funnel Fallout Collectors

\begin{tabular}{|c|c|c|c|c|c|c|c|c|c|c|c|}
\hline \multirow[b]{3}{*}{ Collection } & \multirow{3}{*}{$\begin{array}{c}\text { Days } \\
\text { Exposed }\end{array}$} & \multicolumn{3}{|c|}{ Individual Funnels } & \multirow{3}{*}{$\begin{array}{l}\text { Ratio } \\
\mathrm{Cs} / \mathrm{Sr}\end{array}$} & \multicolumn{6}{|c|}{ Statistics for Each Collection } \\
\hline & & \multirow{2}{*}{$\begin{array}{l}\text { Field } \\
\text { Location }\end{array}$} & \multicolumn{2}{|c|}{$\mathrm{pCi} / \mathrm{m}^{2}$} & & \multicolumn{2}{|c|}{${ }^{137} \mathrm{CS}$} & \multicolumn{2}{|c|}{${ }^{90} \mathrm{Sr}$} & \multicolumn{2}{|c|}{ Ratio } \\
\hline & & & ${ }^{137} \mathrm{Cs}$ & ${ }^{90} \mathrm{Sr}$ & & Mean & $\underline{C V}$ & Mean & $\overline{C V}$ & Mean & $\overline{C V}$ \\
\hline & & $N F$ & -- & 391.4 & -- & & & & & & \\
\hline $6 / 20 / 61-8 / 30 / 61$ & 71 & $C F$ & 157.0 & 504.6 & 0.26 & & & & & & \\
\hline & & SF & & 88.6 & -- & 157 & -- & 362 & 72 & 0.26 & -- \\
\hline $8 / 30 / 61-12 / 1 / 61$ & 93 & NF & 405.8 & 705.9 & 0.57 & & & & & & \\
\hline & & & & -- & & 319 & -- & 106 & -- & 0.51 & -- \\
\hline & & NF & -- & 1974.0 & -- & & & & & & \\
\hline $12 / 1 / 61-2 / 24 / 62$ & 85 & CF & -- & 1799.0 & -- & & & & & & \\
\hline & & SF & 3085.0 & 1208.0 & 2.6 & 3085 & -- & 1660 & 24 & 2.60 & -- \\
\hline $2 / 24 / 62-6 / 21 / 62$ & 117 & $N F$ & 1312.0 & 1021.0 & 1.3 & & & & & & \\
\hline & & CF & -- & 695.7 & -- & 1312 & -- & 858 & -- & 1.30 & -- \\
\hline $6 / 21 / 62-7 / 21 / 62$ & 30 & NF & -- & 228.0 & -- & & & 228 & & & \\
\hline $7 / 21 / 62-8 / 27 / 62$ & 37 & NF & 290.5 & -- & - & & & & & & \\
\hline $1 / 21 / 0<-0 / 21 / 0<$ & & SF & 704.0 & 301.7 & 2.3 & 497 & - & 302 & -- & 2.30 & -- \\
\hline $8 / 27 / 62-10 / 11 / 62$ & 45 & CF & 864.5 & -- & -- & & & & & & \\
\hline (0) & T & SF & 601.4 & 615.7 & 0.97 & 732 & -- & 616 & -- & 0.97 & -- \\
\hline $10 / 11 / 62-11 / 16 / 62$ & 36 & $N F$ & - & 534.5 & - & & & & & & \\
\hline $10 / 11 / 62-11 / 100 / 62$ & 36 & SF & -- & 577.9 & - & -- & -- & 556 & -- & -- & -- \\
\hline
\end{tabular}




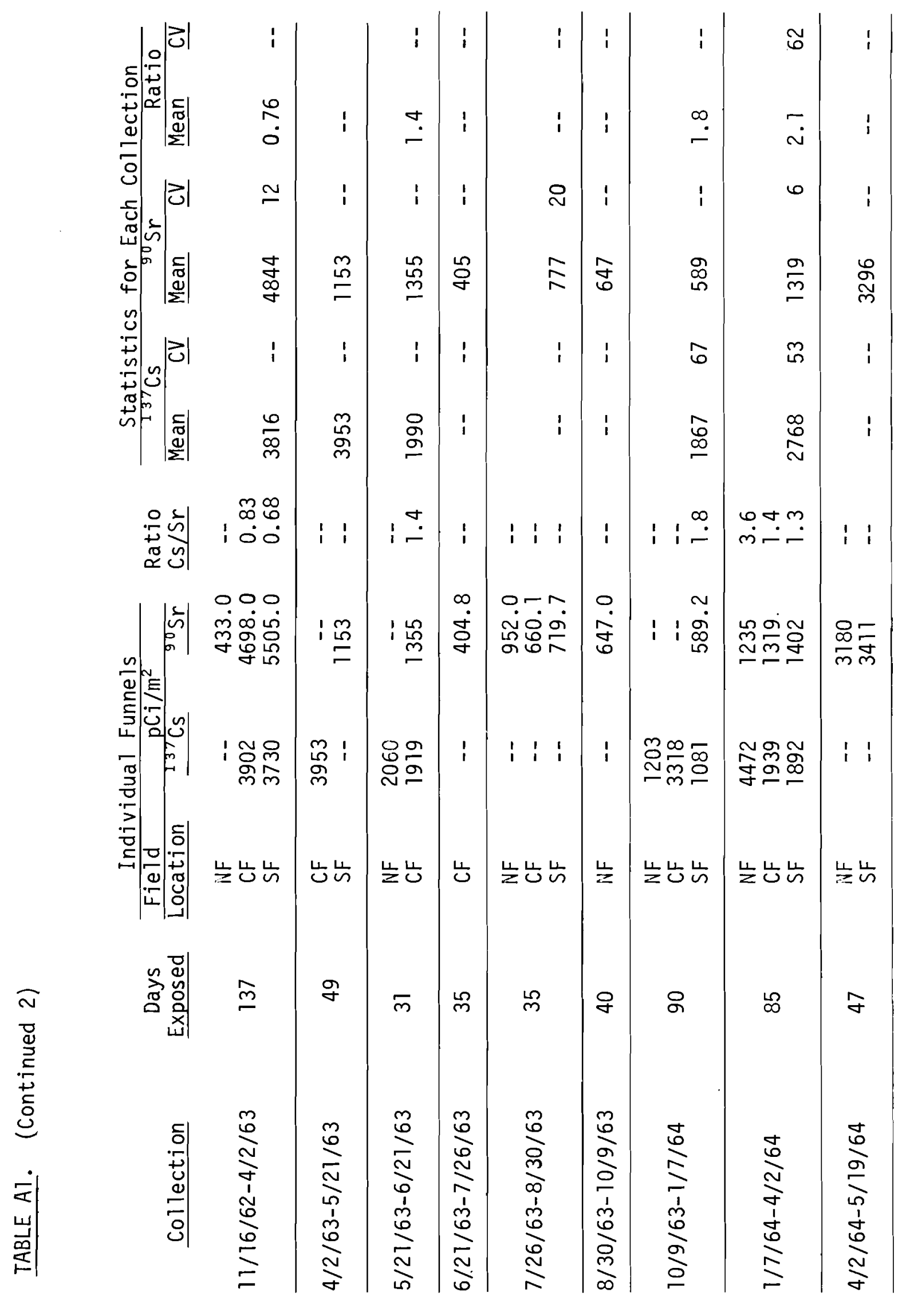


TABLE Al. (Continued 3)

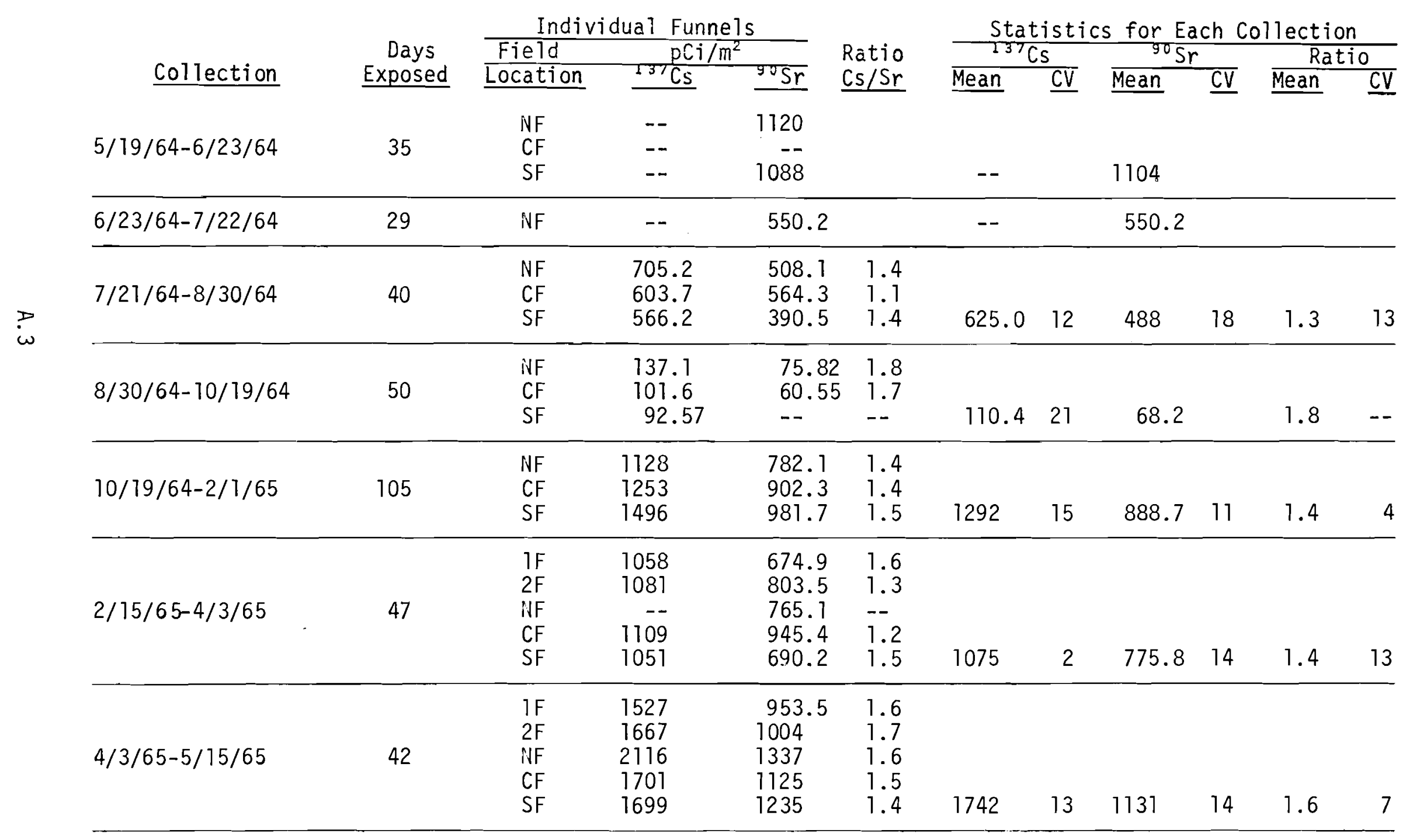


TABLE Al. (Continued 4)

\begin{tabular}{|c|c|c|c|c|c|c|c|c|c|c|c|}
\hline \multirow{3}{*}{ Collection } & \multirow{3}{*}{$\begin{array}{c}\text { Days } \\
\text { Exposed }\end{array}$} & \multicolumn{3}{|c|}{ Individual Funnels } & \multirow{3}{*}{$\begin{array}{l}\text { Ratio } \\
\text { Cs/Sr }\end{array}$} & \multicolumn{6}{|c|}{ Statistics for Each Collection } \\
\hline & & \multirow{2}{*}{\multicolumn{3}{|c|}{ 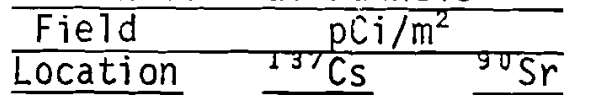 }} & & \multicolumn{2}{|c|}{${ }^{137} \mathrm{Cs}$} & \multirow{2}{*}{\multicolumn{2}{|c|}{${ }^{90} \mathrm{Sr}$}} & \multirow{2}{*}{\multicolumn{2}{|c|}{$\frac{\text { Ratio }}{\text { Mean CV }}$}} \\
\hline & & & & & & Mean & CV & & & & \\
\hline \multirow{4}{*}{$5 / 15 / 65-6 / 23 / 65$} & \multirow{4}{*}{39} & $1 F$ & $-\overline{7}$ & 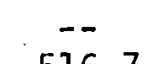 & & & & & & & \\
\hline & & NF & $\begin{array}{l}80.1 \\
893.9\end{array}$ & 510.7 & 1.6 & & & & & & \\
\hline & & $\mathrm{CF}$ & $1040^{\circ}$ & 617.5 & 1.7 & & & & & & \\
\hline & & SF & -- & 503.2 & - & 913.9 & 13 & 545.8 & 11 & 1.6 & \\
\hline \multirow{3}{*}{$6 / 23 / 65-7 / 25 / 65$} & \multirow{3}{*}{32} & $1 \mathrm{~F}$ & 197.7 & 84.91 & 2.3 & & & & & & \\
\hline & & $2 \mathrm{~F}$ & 279.7 & 136.4 & 2.1 & & & & & & \\
\hline & & Nit & 232.3 & 136.8 & 1.1 & 2070 & & 7170 & & 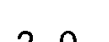 & \\
\hline \multirow{6}{*}{$7 / 25 / 65-8 / 31 / 65$} & \multirow{6}{*}{37} & & & & & & & & & & \\
\hline & & $1 \mathrm{~F}$ & 185.7 & 133.0 & 1.4 & & & & & & \\
\hline & & $2 \mathrm{~F}$ & 197.0 & 112.2 & 1.7 & & & & & & \\
\hline & & $\mathrm{NF}$ & 221.9 & 122.5 & 1.8 & & & & & & \\
\hline & & $\begin{array}{l}C F \\
\text { SF }\end{array}$ & 209.2 & 99.42 & 2.1 & 2030 & & & & & \\
\hline & & $\mathrm{st}$ & 201.0 & 123.2 & 1.6 & 203.0 & 1 & 118.1 & 10 & 1.1 & 15 \\
\hline \multirow{4}{*}{$8 / 31 / 65-10 / 10 / 65$} & \multirow{4}{*}{40} & $\begin{array}{l}1 \mathrm{~F} \\
2 \mathrm{~F}\end{array}$ & $\begin{array}{l}76.81 \\
79.92\end{array}$ & $\begin{array}{l}99.80 \\
45.82\end{array}$ & .8 & & & & & & \\
\hline & & NF & 1033 & -- & - & & & & & & \\
\hline & & CF & 67.69 & -- & -- & & & & & & \\
\hline & & SF & 211.3 & -- & -- & 293.7 & 142 & 72.81 & & 1.2 & \\
\hline \multirow{4}{*}{$11 / 11 / 65-4 / 1 / 66$} & \multirow{4}{*}{141} & $1 \mathrm{~F}$ & 510.1 & 302.5 & 1.7 & & & & & & \\
\hline & & $2 F$ & 528.2 & 332.5 & 1.6 & & & & & & \\
\hline & & Tr & ? & 2332 & 10 & & & & & & \\
\hline & & SF & $\begin{array}{l}392.6 \\
\end{array}$ & 283.9 & 1.4 & 468.2 & 13 & 285.5 & & 1.6 & 13 \\
\hline
\end{tabular}


TABLE Al. (Continued 5)

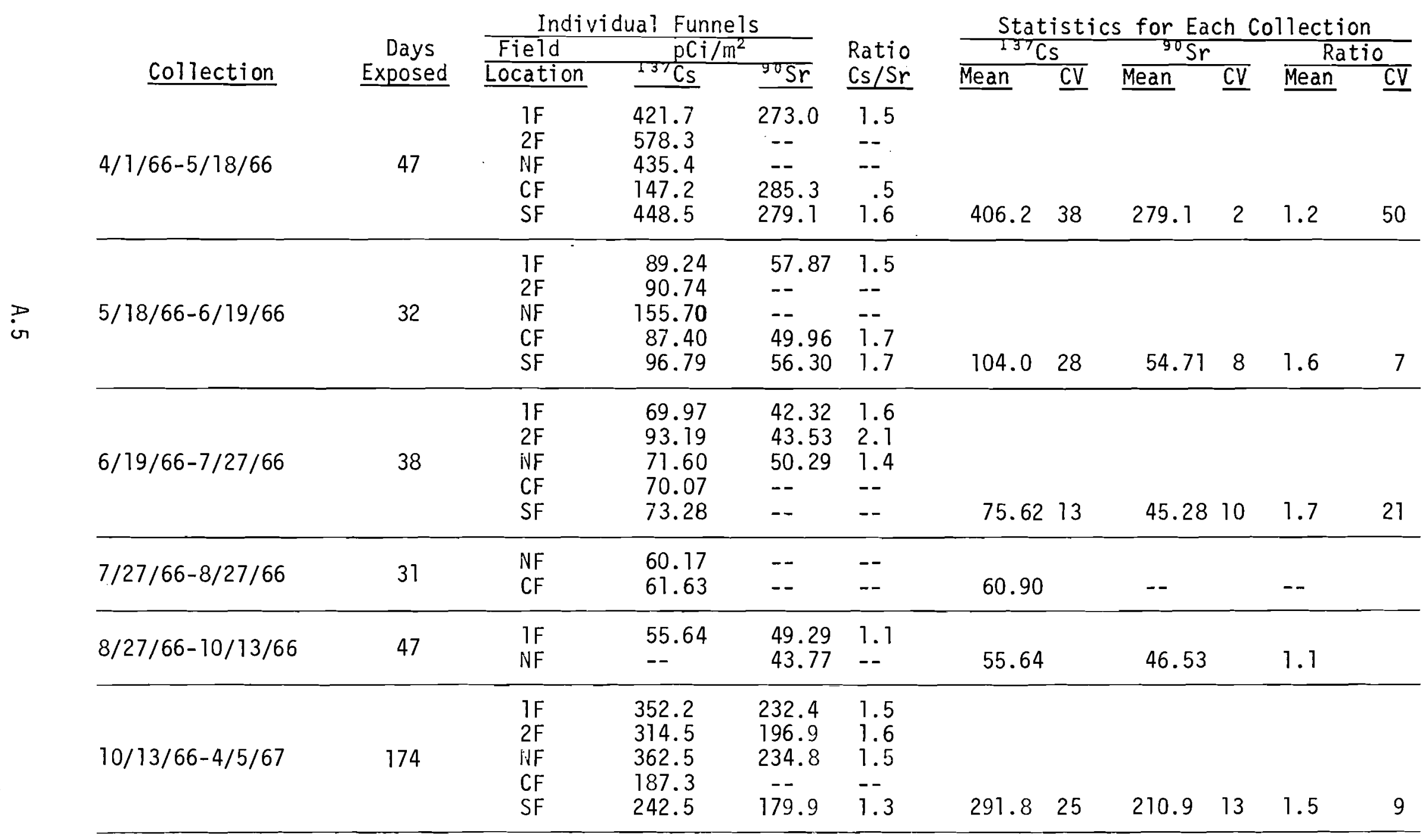


TABLE A1. (Continued 6)

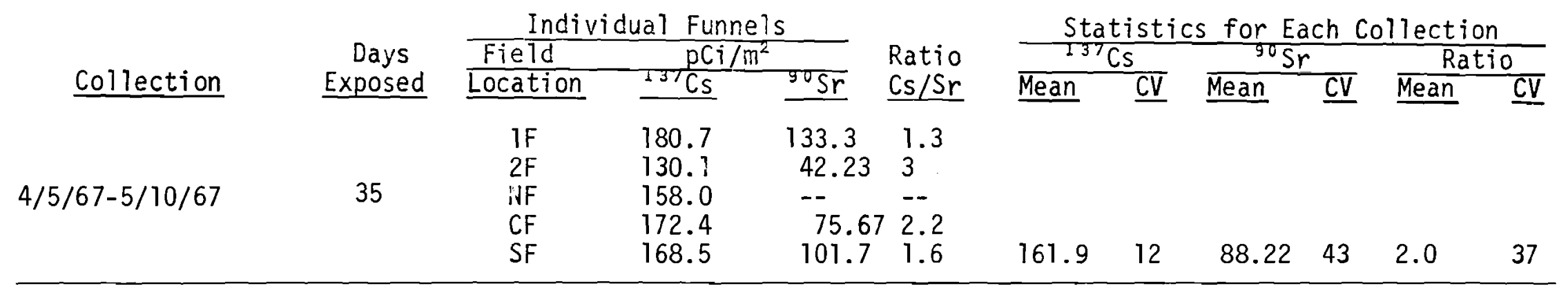




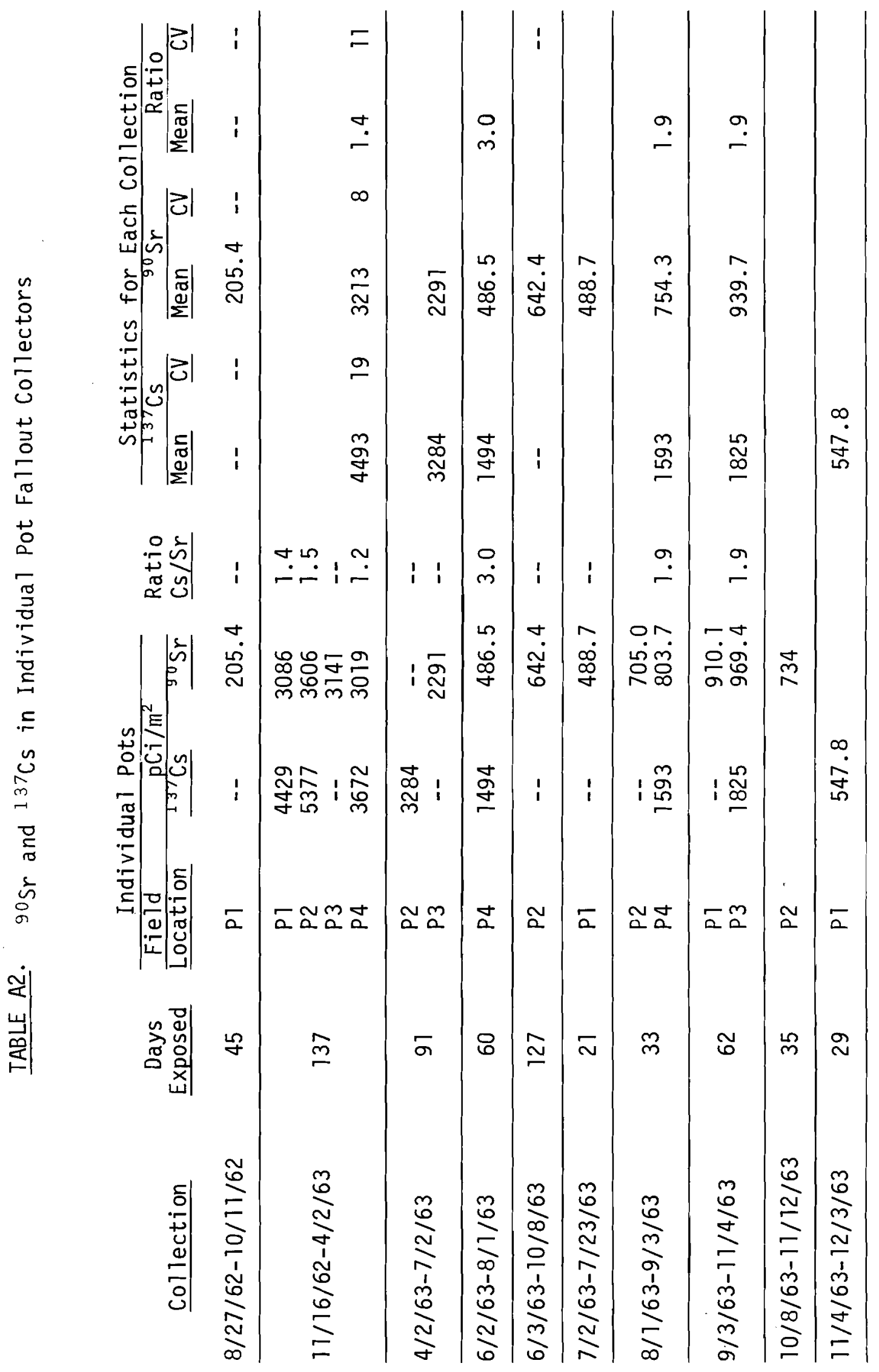

A. 7 
TABLE A2. (Continued 2)

\begin{tabular}{|c|c|c|c|c|c|c|c|c|c|c|c|}
\hline \multirow[b]{3}{*}{ Collection } & \multirow{3}{*}{$\begin{array}{c}\text { Days } \\
\text { Exposed }\end{array}$} & \multicolumn{3}{|c|}{ Individual Pots } & \multirow{3}{*}{$\begin{array}{l}\text { Ratio } \\
\mathrm{Cs} / \mathrm{Sr} \\
\end{array}$} & \multicolumn{6}{|c|}{ Statistics for Each Collection } \\
\hline & & \multirow{2}{*}{$\begin{array}{l}\text { Field } \\
\text { Location }\end{array}$} & \multicolumn{2}{|c|}{$\frac{\mathrm{pCi} / \mathrm{m}^{2}}{{ }^{90} \mathrm{Sr}}$} & & ${ }^{137} \mathrm{C}$ & & ${ }^{0} s r$ & & & \\
\hline & & & ${ }^{137} \mathrm{Cs}$ & ${ }^{90} \mathrm{Sr}$ & & Mean & $\underline{\mathrm{CV}}$ & Mean & $\underline{\mathrm{CV}}$ & $\overline{\text { Mean }}$ & $\overline{C V}$ \\
\hline $11 / 12 / 63-1 / 6 / 63$ & 55 & $\begin{array}{l}\text { P2 } \\
\text { P4 }\end{array}$ & $\begin{array}{l}580.3 \\
1953\end{array}$ & $\begin{array}{l}228.9 \\
226.9\end{array}$ & $\begin{array}{l}2.5 \\
8.6\end{array}$ & 1267 & & 227.8 & & 5.5 & \\
\hline $1 / 6 / 64-4 / 2 / 64$ & 86 & $\begin{array}{l}\text { P1 } \\
\text { P2 } \\
\text { P3 } \\
\text { P4 }\end{array}$ & $\begin{array}{l}1704 \\
1860 \\
2125 \\
1823\end{array}$ & $\begin{array}{l}1171 \\
1168 \\
1229 \\
1233\end{array}$ & $\begin{array}{l}1.4 \\
1.6 \\
1.7 \\
1.5\end{array}$ & 1878 & 9 & 1200 & 3 & 1.6 & 8 \\
\hline $4 / 2 / 64-5 / 15 / 64$ & 43 & $\begin{array}{l}\text { P2 } \\
\text { P4 }\end{array}$ & $\begin{array}{l}4818 \\
4295\end{array}$ & $\begin{array}{l}2796 \\
2800\end{array}$ & $\begin{array}{l}1.7 \\
1.5\end{array}$ & 4557 & & 2798 & & 1.6 & \\
\hline $4 / 2 / 64-7 / 6 / 64$ & 95 & $\begin{array}{l}\text { P1 } \\
\text { P3 }\end{array}$ & $6 \overline{743}$ & $\begin{array}{l}3702 \\
4003\end{array}$ & $\overline{1.7}$ & 3853 & & 6743 & & 1.7 & \\
\hline $5 / 15 / 64-7 / 1 / 64$ & 47 & $\begin{array}{l}\text { P2 } \\
\text { P4 }\end{array}$ & $\begin{array}{l}2001 \\
2074\end{array}$ & $\begin{array}{c}1309 \\
--\end{array}$ & 1.5 & 2038 & & 1309 & & 1.5 & \\
\hline $7 / 1 / 64-8 / 4 / 64$ & 34 & $\begin{array}{l}\text { P2 } \\
\text { P4 }\end{array}$ & $\begin{array}{l}538 \\
509.1\end{array}$ & $\overline{353}$ & $\overline{1.4}$ & 523 & & & & 1.4 & \\
\hline $8 / 4 / 64-9 / 1 / 64$ & 28 & $\begin{array}{l}\text { P2 } \\
\text { P4 }\end{array}$ & $\begin{array}{l}239.7 \\
254.7\end{array}$ & $\begin{array}{l}172.8 \\
173.8\end{array}$ & $\begin{array}{l}1.3 \\
1.4\end{array}$ & 246.9 & & 173.3 & & 1.3 & \\
\hline $9 / 1 / 64-10 / 1 / 64$ & & $\begin{array}{l}\text { P2 } \\
\text { P4 }\end{array}$ & $\begin{array}{r}93.6 \\
105.9\end{array}$ & $\begin{array}{l}59.88 \\
64.24\end{array}$ & $\begin{array}{l}1.5 \\
1.6\end{array}$ & 99.75 & & 62.06 & & 1.5 & \\
\hline $9 / 6 / 64-2 / 1 / 65$ & & $\begin{array}{l}\text { P1 } \\
\text { P3 }\end{array}$ & $\begin{array}{l}2166 \\
1984\end{array}$ & $\begin{array}{l}1263 \\
1270\end{array}$ & $\begin{array}{l}1.7 \\
1.6\end{array}$ & 2075 & & 1267 & & 1.6 & \\
\hline
\end{tabular}


TABLE A2. (Continued 3)

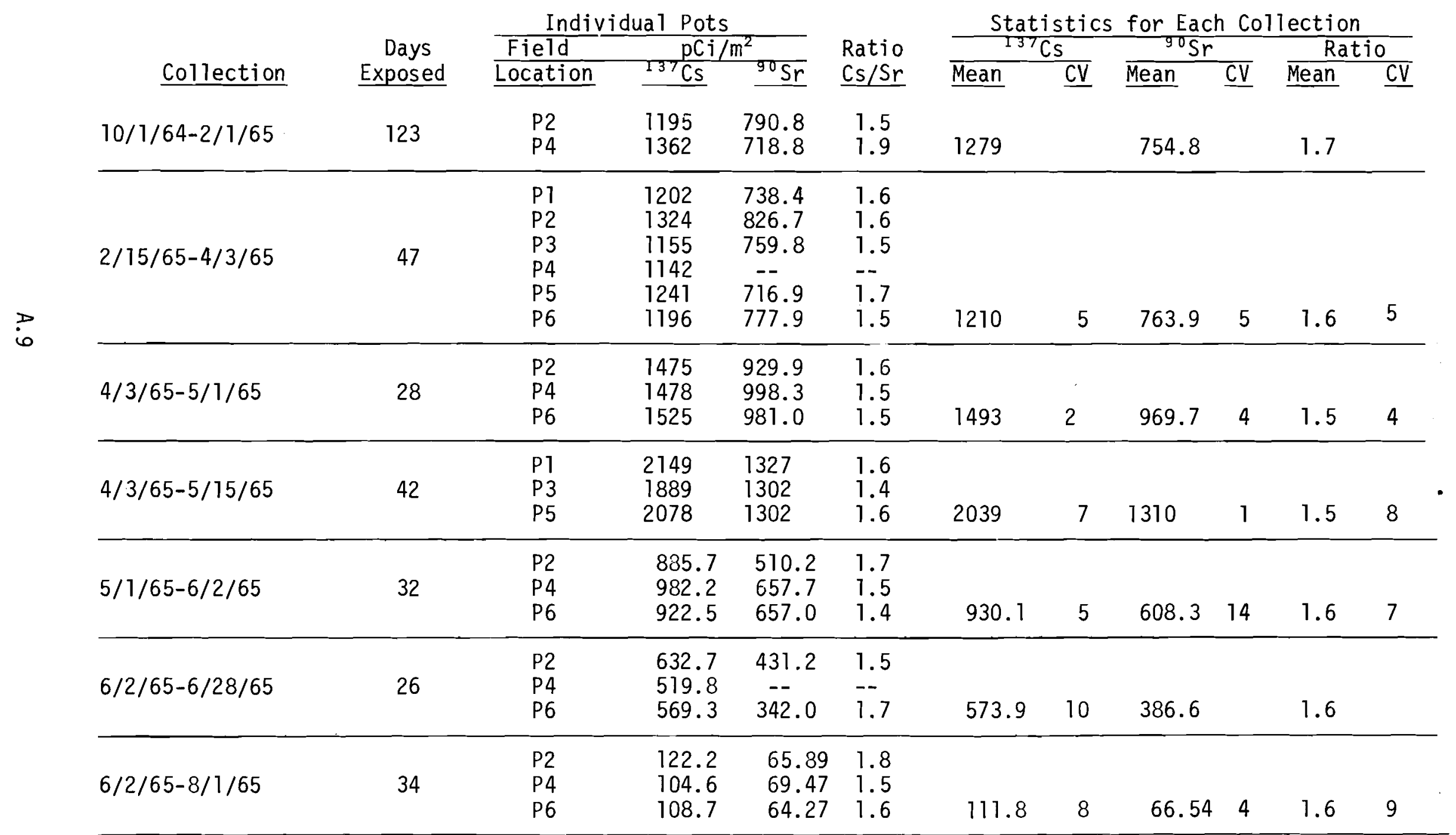


TABLE A2. (Continued 4)

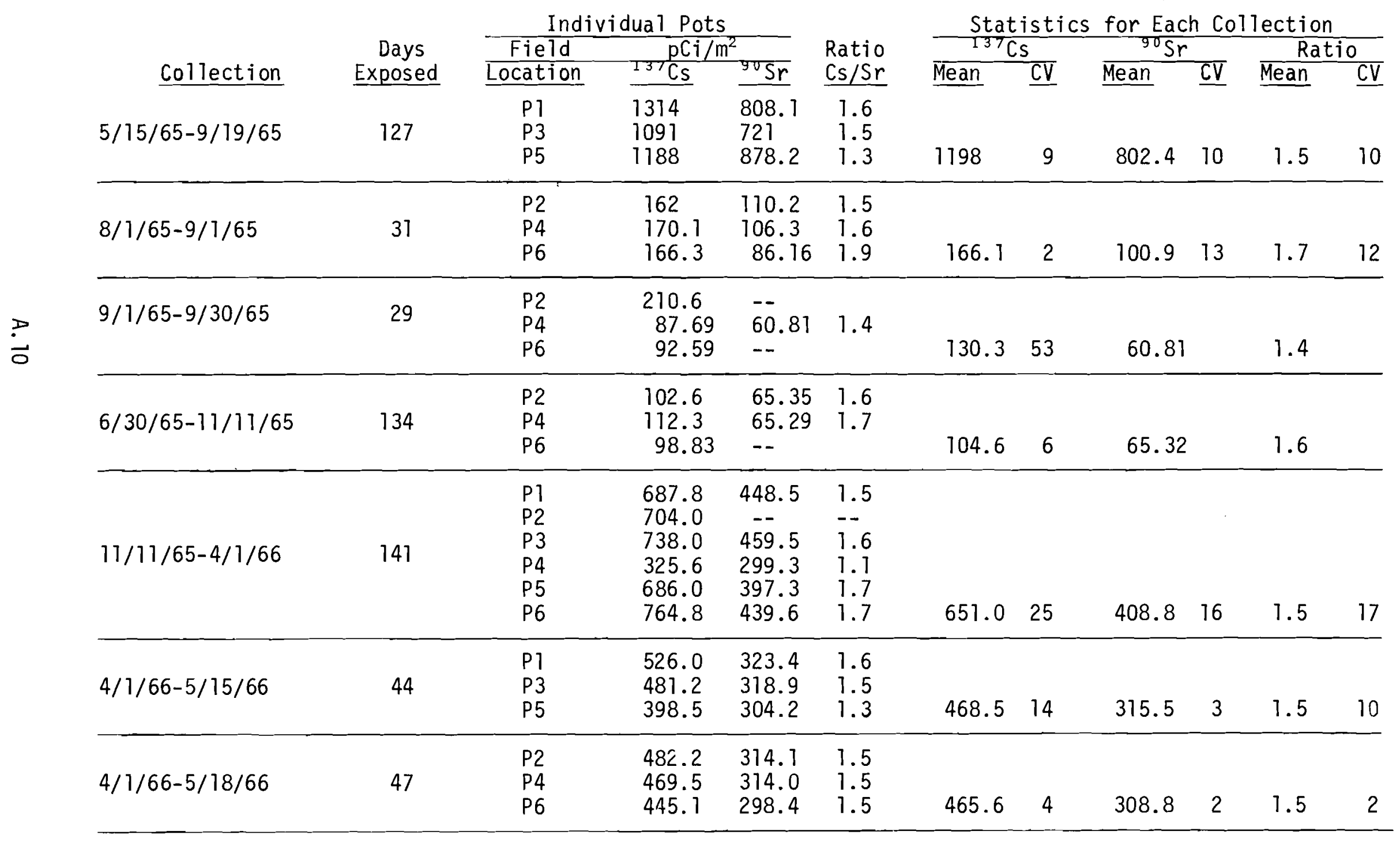




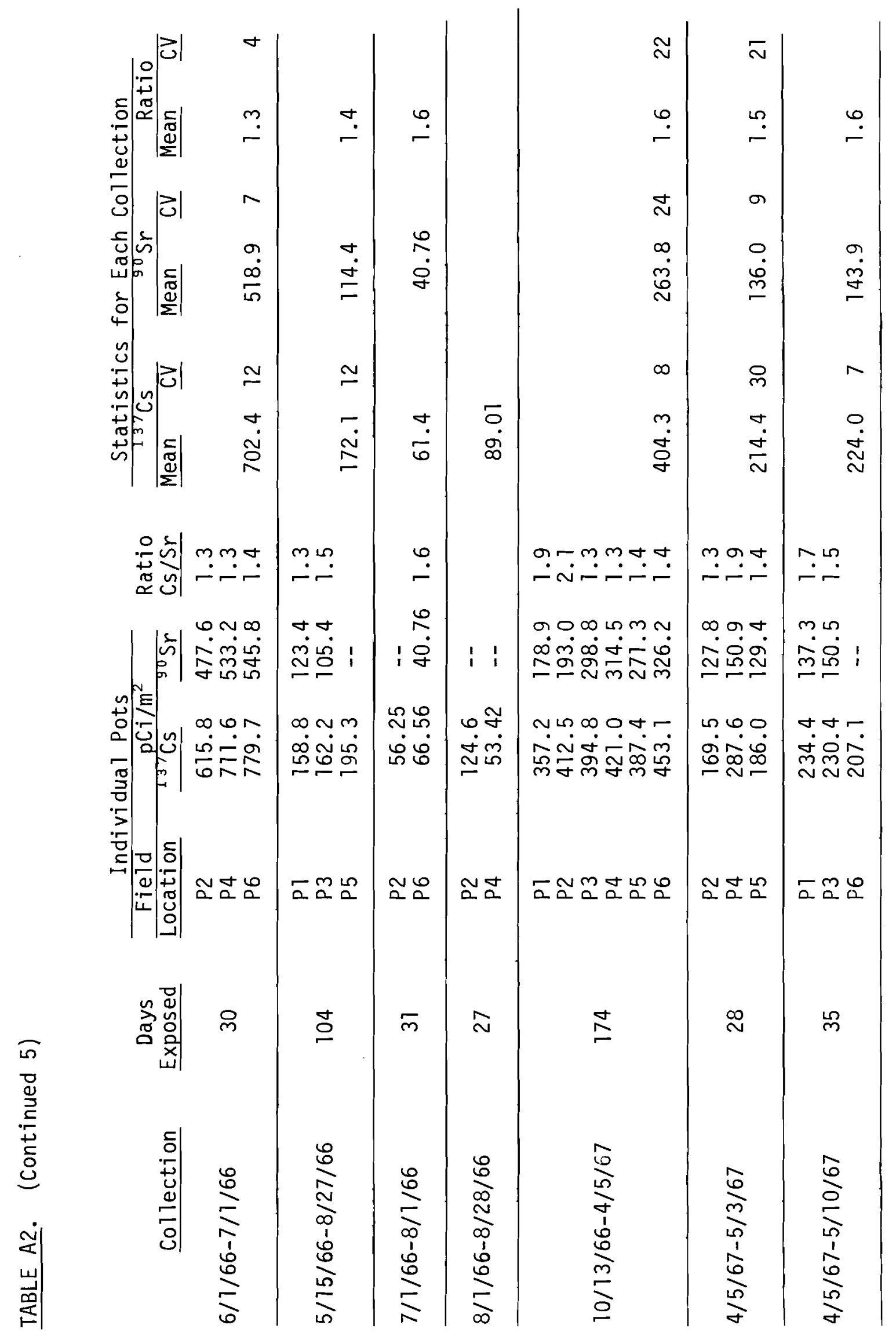


TABLE A3. Mean Monthly Deviation From the 29 Year

Mean of the Daily Temperature Range at

Saint George Power House

Month

Year

\begin{tabular}{|c|c|c|c|c|c|c|c|}
\hline & $61-62$ & $62-63$ & $63-64$ & $\underline{64-65}$ & $\underline{65-66}$ & $\underline{66-67}$ & Norma $1^{1}$ \\
\hline November & -0.5 & 4.2 & 4.3 & -1.8 & 3.0 & 5.4 & 48.0 \\
\hline December & -1.5 & -0.1 & -1.5 & 1.6 & 0.2 & 1.4 & 40.8 \\
\hline January & 0.02 & -3.8 & -1.2 & 5.4 & -1.7 & 1.6 & 39.2 \\
\hline February & 3.2 & 6.1 & -1.7 & 1.8 & -3.8 & 2.9 & 44.5 \\
\hline March & -3.6 & -0.5 & -3.1 & -1.6 & 2.1 & 4.5 & 51.8 \\
\hline April & 4.9 & -4.0 & -1.5 & -1.9 & 0.8 & -5.2 & 60.5 \\
\hline May & -2.1 & 3.3 & -1.0 & -2.6 & 3.9 & 1.0 & 68.3 \\
\hline June & 0.03 & -2.5 & 0.10 & -2.5 & 3.2 & -0.5 & 76.6 \\
\hline JuTy & -1.3 & -1.3 & 2.2 & -0.3 & 1.5 & 4.7 & 83.7 \\
\hline August & 1.0 & -0.3 & 0.7 & -0.6 & 3.6 & 4.6 & 82.1 \\
\hline September & 2.6 & 0.3 & -0.6 & -5.6 & 3.7 & 2.3 & 74.7 \\
\hline October & 2.0 & 3.1 & 5.5 & 0.9 & 2.3 & 4.1 & 62.1 \\
\hline
\end{tabular}

${ }^{1}$ Normals are climatological normals based on the period 1931-1960. Daily average maximum and minimum temperatures are used to calculate monthly averages. Monthly maximum and minimum values are averaged aga in to obtain the monthly average temperature. The tabled values result when the average temperature is compared to the climatological normal above. 

TABLE A4. Mean Monthly Deviation From The 29 Year Mean of The Daily Precipitation at Saint George Power House

Month

\begin{tabular}{|c|c|c|c|c|c|c|}
\hline & & Years & & & & Normal ${ }^{1}$ \\
\hline $61-62$ & $62-63$ & $63-64$ & $64-65$ & $65-66$ & $66-67$ & Inches \\
\hline
\end{tabular}

November

$0.27-0.28$

0.01

$-0.10$

1.55

0.07

0.58

December $-0.57$

$-0.77$

$-1.03$

$-0.56$

1.22

0.74

1.03

January

$-0.31$

$-0.15$

$-0.67$

$-0.72$

$-0.42$

0.48

0.98

February

0.08

0.40

$-1.03$

0.01

$-0.59$

$-1.03$

1.03

March

$-0.37$

$\begin{array}{ll}-0.25 & -0.10\end{array}$

0.09

$-0.89$

$-0.77$

0.91

April

$-0.45$

0.16

0.28

$1.86-0.10$

0.22

0.48

May

$-0.29$

$-0.38$

0.41

0.22

$-0.26$

$-0.20$

0.39

June

$-0.08$

$-0.21$

0.07

$-0.10$

$-0.17$

$-0.05$

0.24

July

$\begin{array}{ll}-0.54 & -0.45\end{array}$

0.45

$-0.39$

$-0.17$

$-0.37$

0.60

Augus $t$

$-0.59$

$0.73 \quad-0.01$

0.38

$-0.57$

$-0.24$

0.61

September

$-0.06$

0.76

$-0.59$

$-0.30$

$-0.13$

1.42

0.60

October

$-0.26$

$-0.43$

$-0.68$

$-0.50$

$-0.43$

$-0.68$

0.68

Total

$-3.17$

$-0.87$

$-2.89$

$-0.05 \quad-0.96$

$-0.41$

8.13

Normals are based on averages for the period 1931-1960. 
in o oco 00000030000000000000000004 mo0000000000000000000000000003

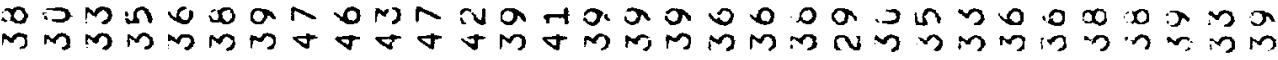

$\infty \propto$

is

$\alpha \mu$

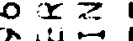

$\rightarrow 12=$

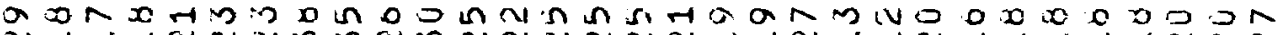
$\sum_{11}^{2}$

$+\underset{<}{x}$

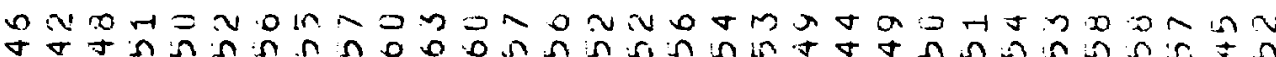
$\sum$

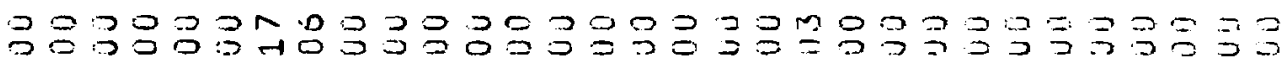

$\underset{\sim}{\sim}:$

$\underset{1}{\pi}:$

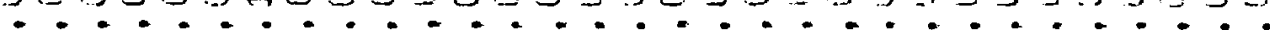

¿े

$\geq$

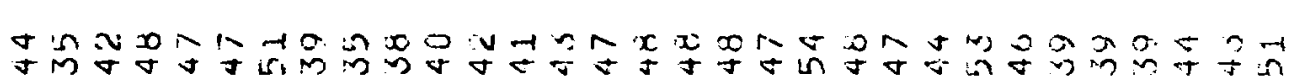

$\frac{1}{5}$

(

n $x$.

$x \geq$

$\rightarrow \underset{\Sigma}{2} \vec{\Sigma}$

$\Sigma$

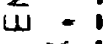

$+$

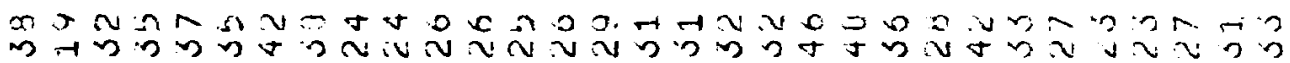

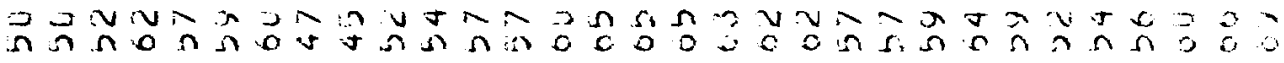

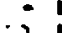

:

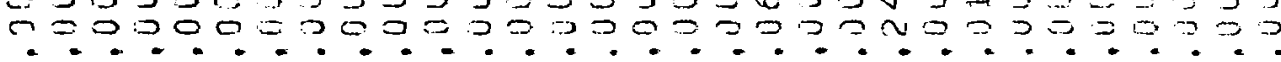

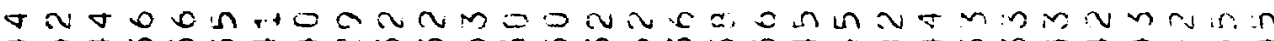

$\mu \stackrel{2}{<}$ \pm in

ه '

穵

$-$

$\approx$

1

a

$+\frac{1}{x}$

I $2>1$

a 11

을 웜

$+1$

$\because 1$

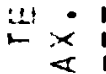

i. $\frac{2}{2}$

$>\leq \quad \leq$

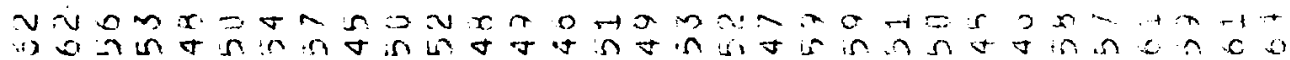

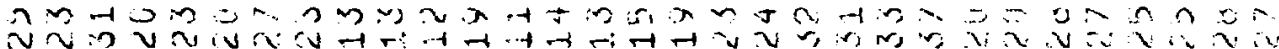

$-1$

a

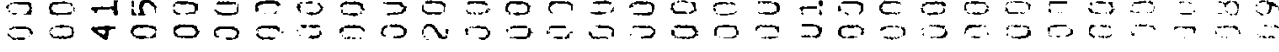

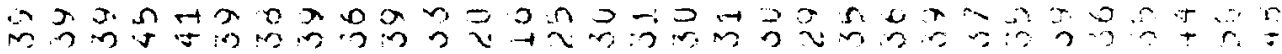

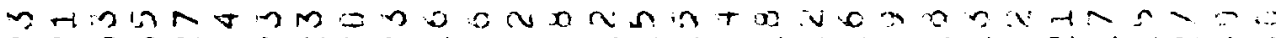

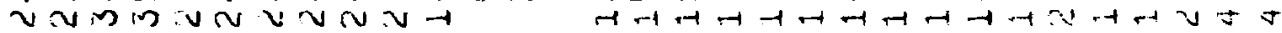

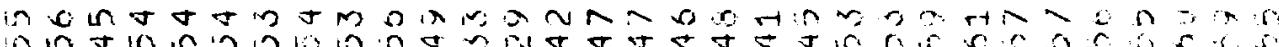

$\approx$

hNm

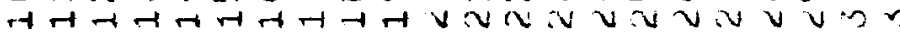




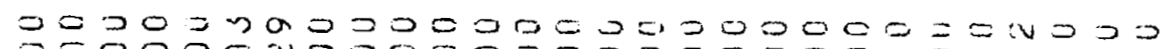

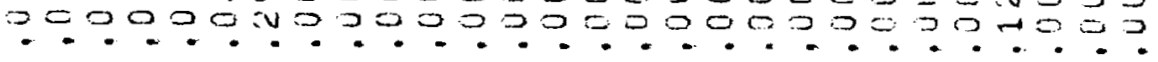

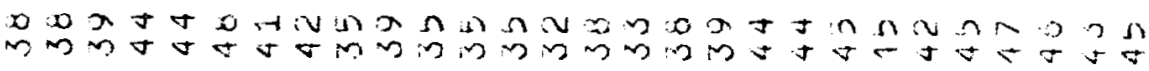
.$\div$ $0 \geq \geq$

$\rightarrow+\infty=$

:

$\stackrel{i}{i} \dot{x}$

x

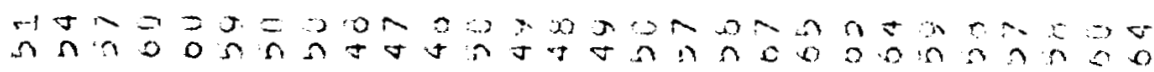

2
$\approx$

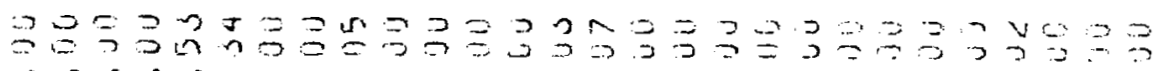

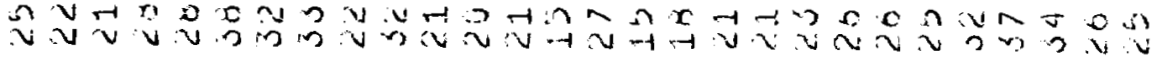

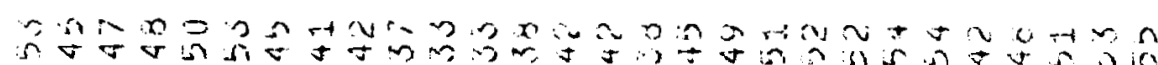

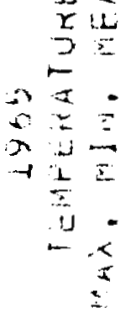

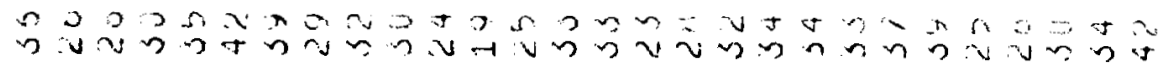

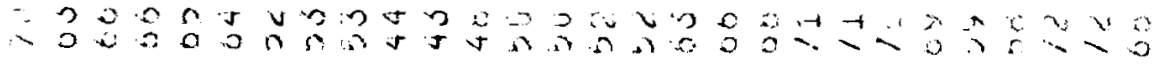

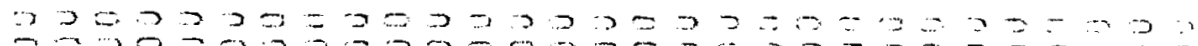

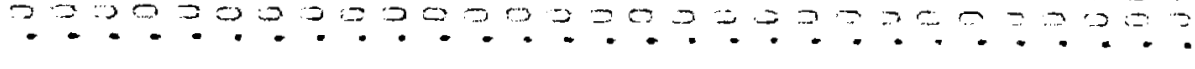

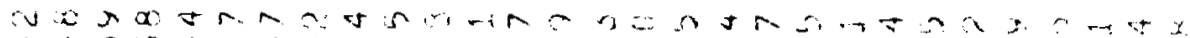
$\checkmark \div$

c $x \rightarrow$ i

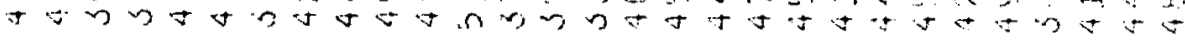

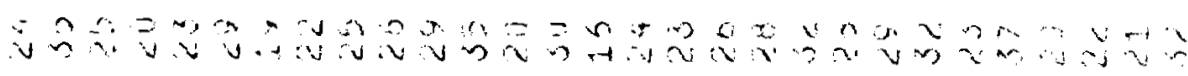
$\therefore \rightarrow$

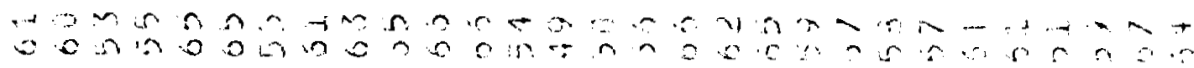

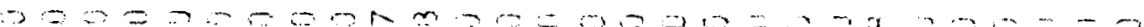

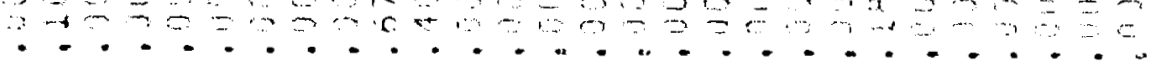

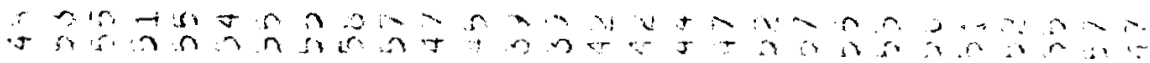

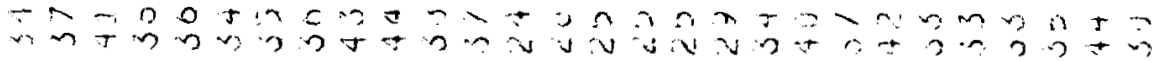

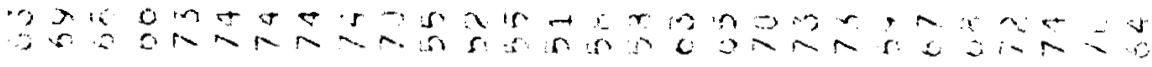

- 


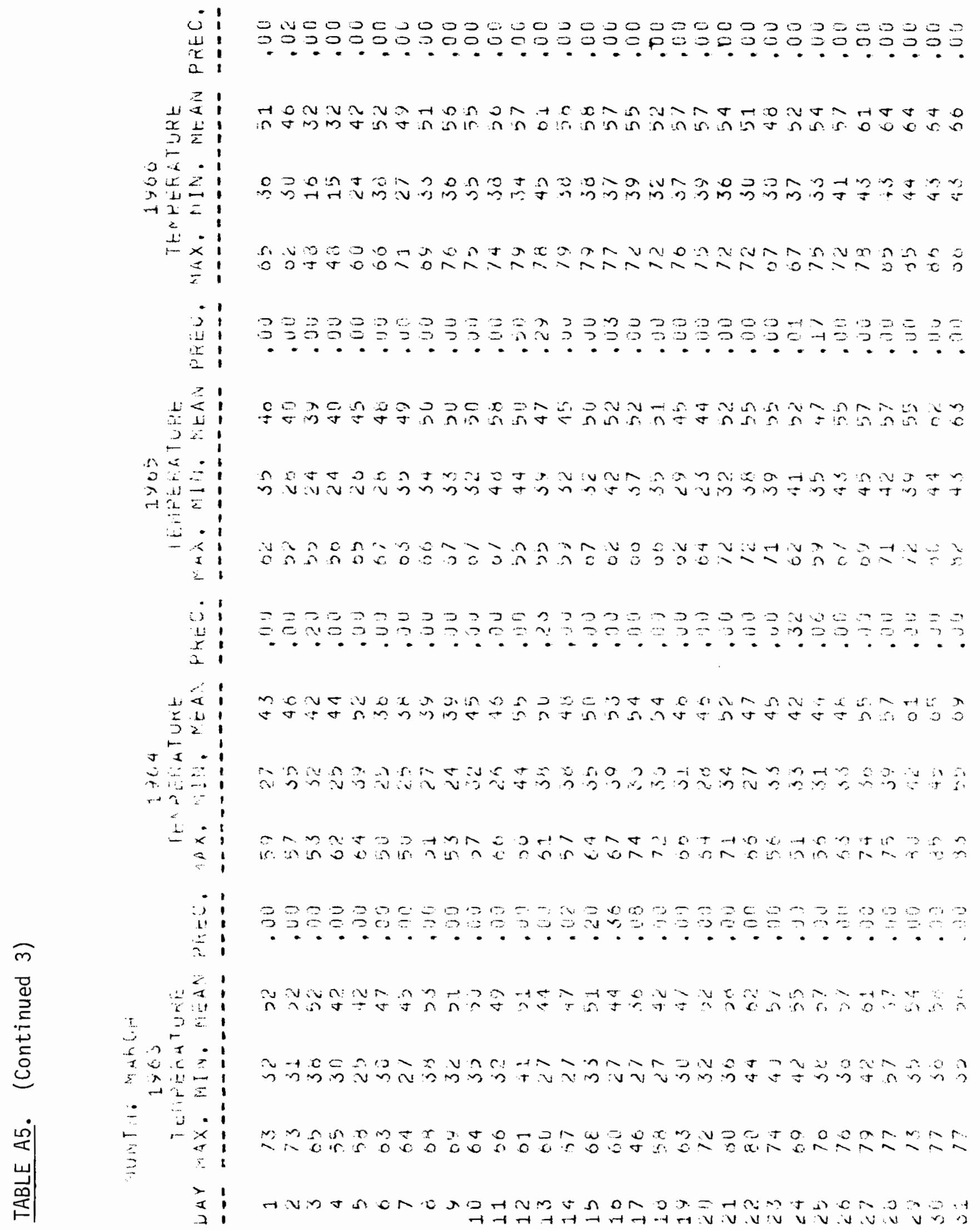


崩

a :

岒变

I

$0 \stackrel{1}{x}$.

b $x \geq 1$

o. $u=1$

C.

$i$;

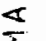

$\therefore 1$

is

a :

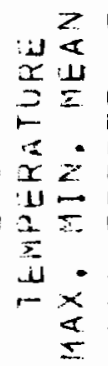

$\begin{array}{r}\square \\ 0 \\ \frac{1}{2} \\ .5 \\ \frac{1}{5} \\ \hline\end{array}$

-

L

京

2 (5) :11

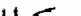
$=\geq \ldots$

$r 2 x \div 1$

$x$ o $x \geq$

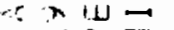
. $\rightarrow 2$ i 2

$0000000=000000000 m+x 0000000000$

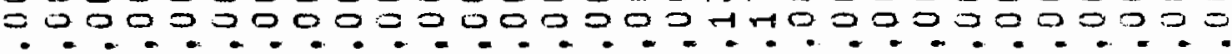

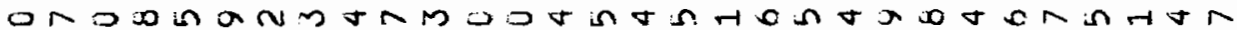

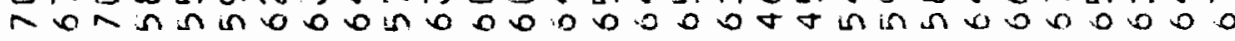

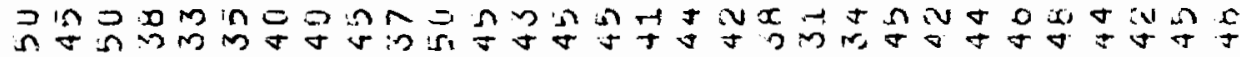

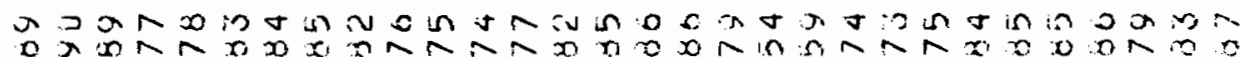

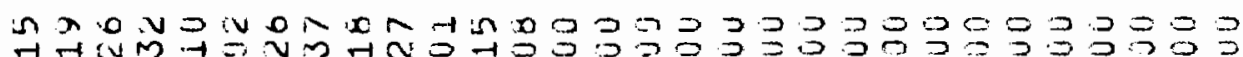

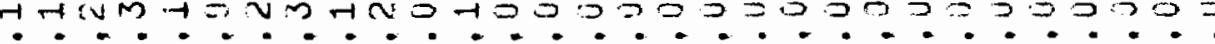

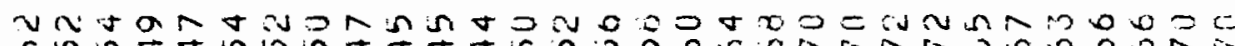

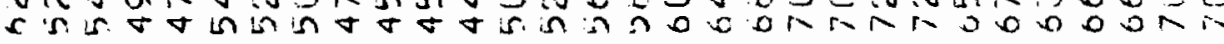

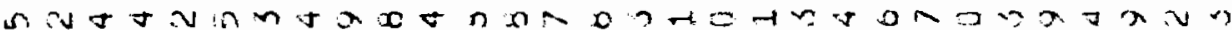

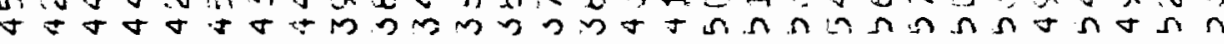

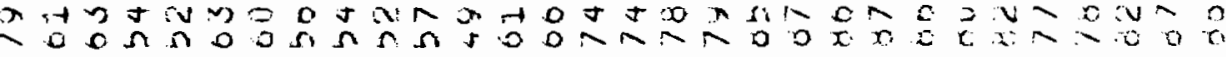

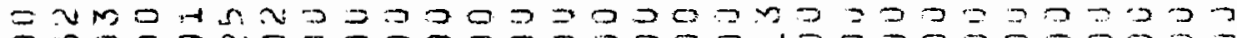

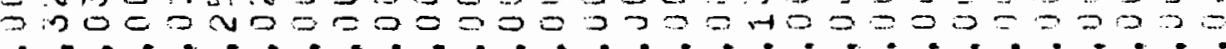

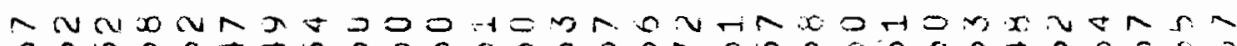

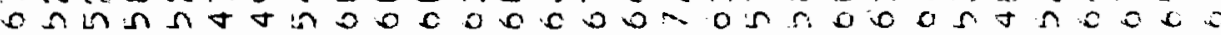

$\begin{array}{ll}5 & a \\ \pi & 0\end{array}$

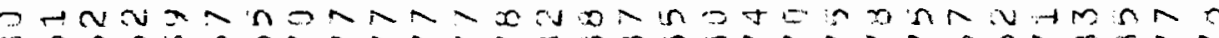

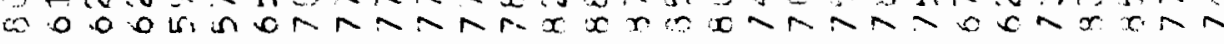

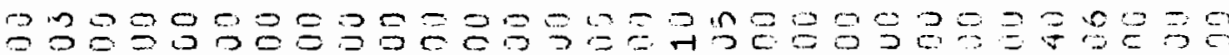

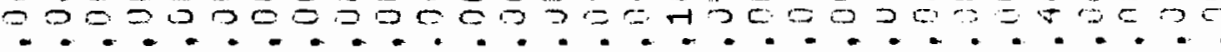

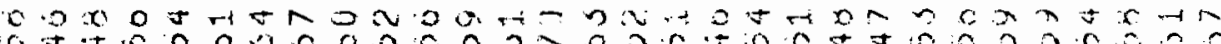

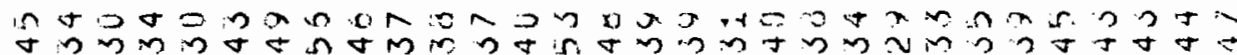

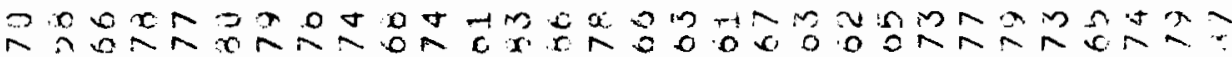

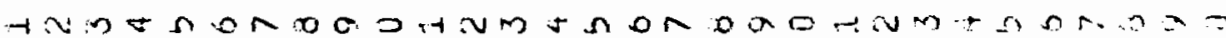

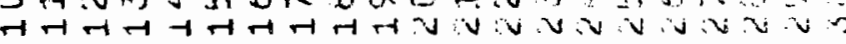




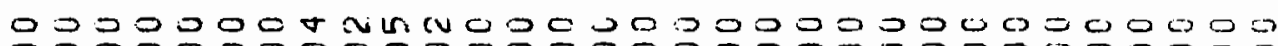
ว

z

¿山! 5

c

$2 x \geq$

$\rightarrow \frac{1}{2}=$

定

$\dot{5}$

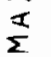

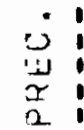

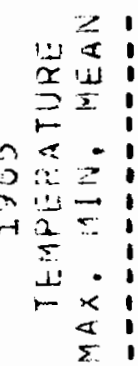

is

id

21

让 2

号:

$\sigma$

$\therefore \approx$

T.

$\rightarrow 2$

i.

$-x$

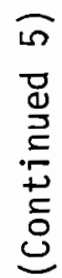

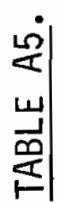
$>n<-1$

a JNN

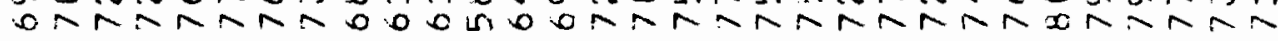

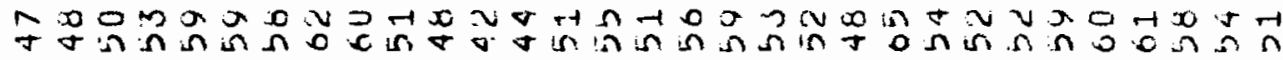

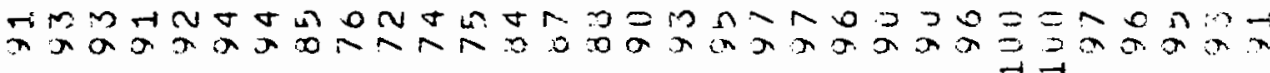

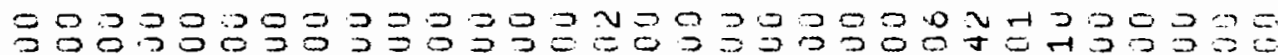

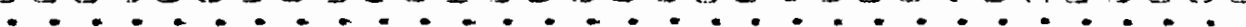

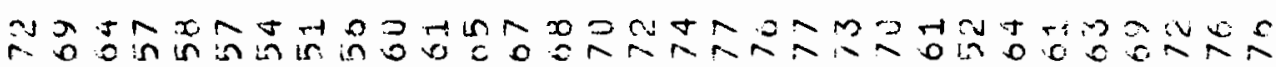

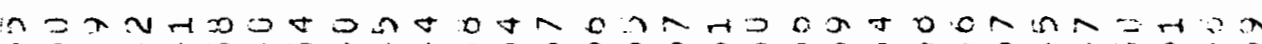

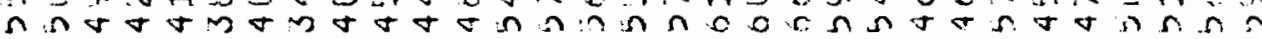

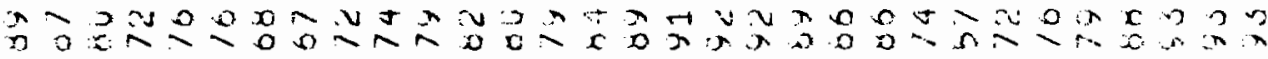

$=200000+20000200920=0: 00002020=$

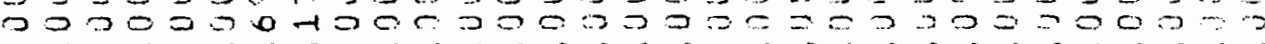

x mt

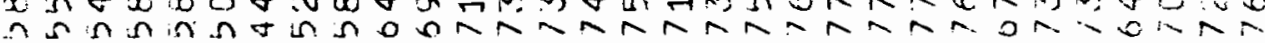

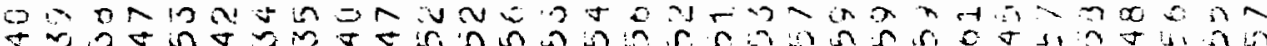

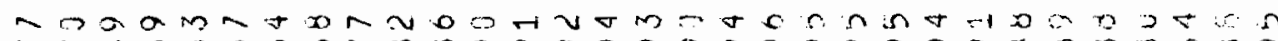
人)

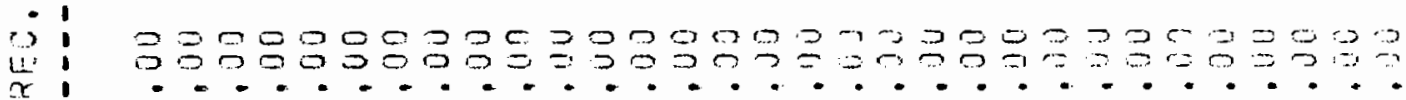

क

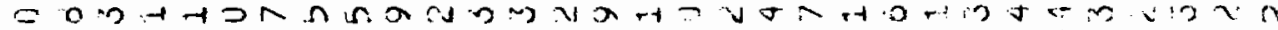

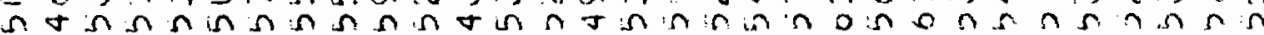

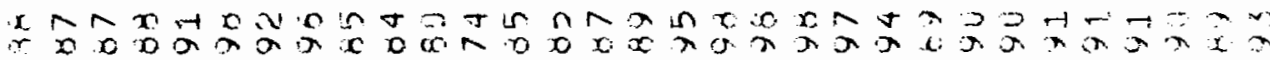

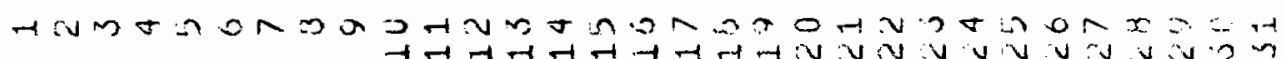


$00=30000000000000000000000000 N$ 崩: $: 00000000000000009000000000000$

a.

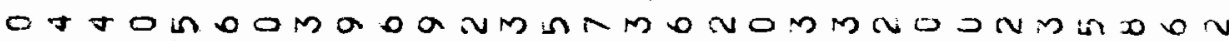

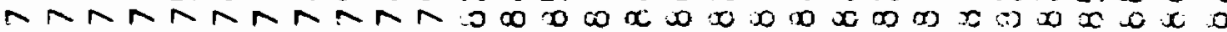

$\omega<$

$\stackrel{L}{\Sigma}$ $\rightarrow \div$

$\dot{x}$ 0. $x \geq$

$+\frac{1}{x} \leq$

[i].

$-x$

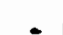

$\underbrace{0}_{-15}$

崩

‥

$\geq 1$

ii $x$

等

n $x$

ง $\simeq$

$2.11=$

$+\frac{1}{2}$

$\sum$

H

5

:

业

$x$

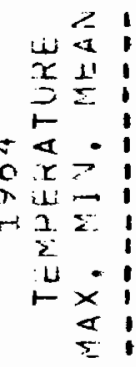

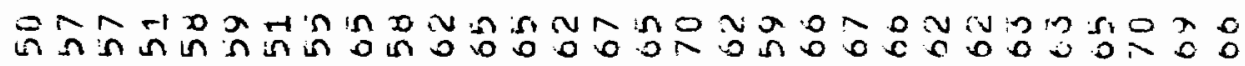

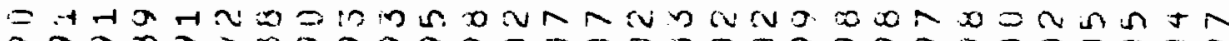

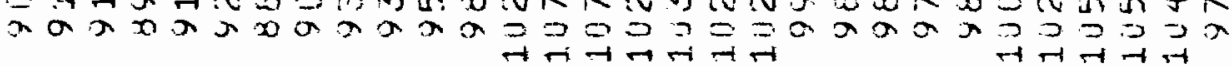

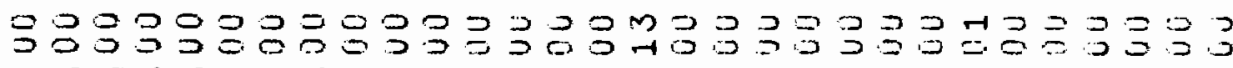
.

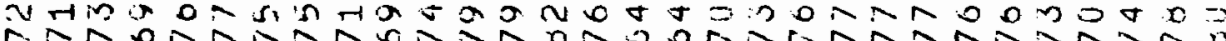

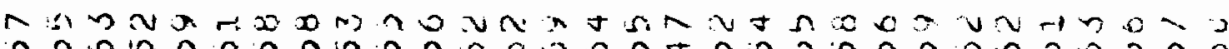

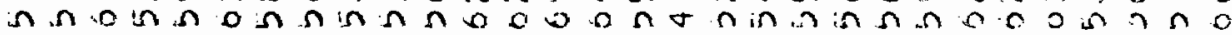

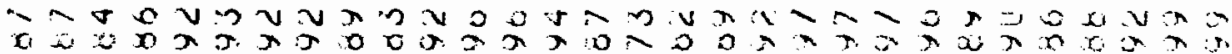

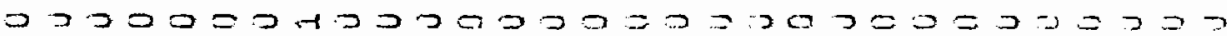

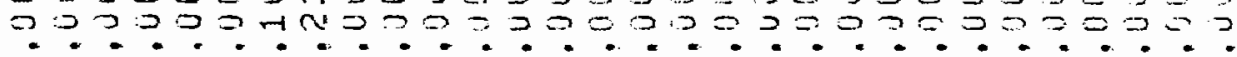

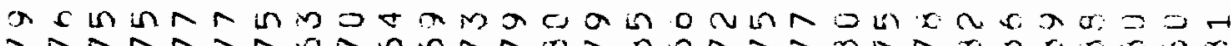

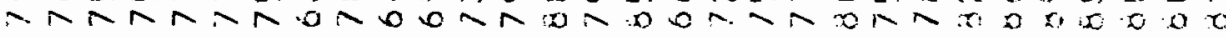

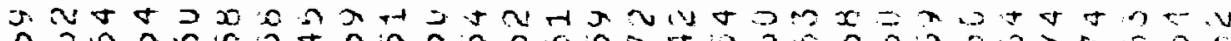

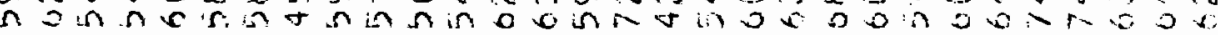

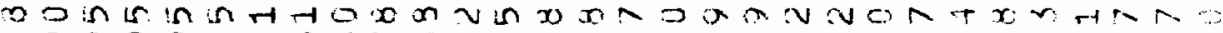

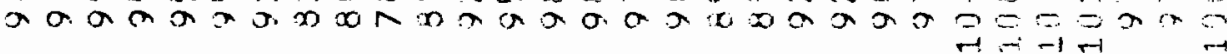

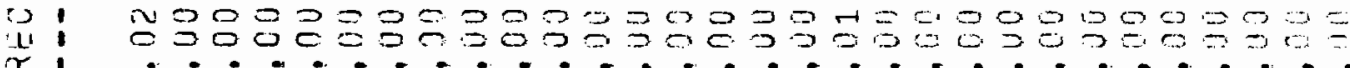

a

$\sin =0$.

(1)

$\underline{\underline{3}}$

$\because 2 x$

$\div:$ $\rightarrow 2=$

$\ddot{I}$
$z$
$z$ is

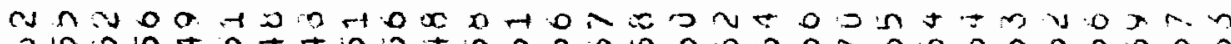
$\Rightarrow$ in in $\ln \theta$ a t in

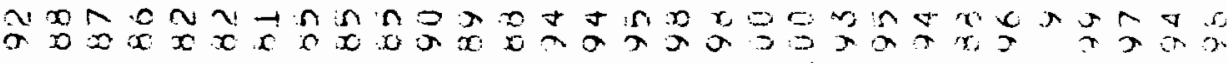

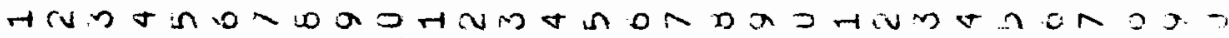

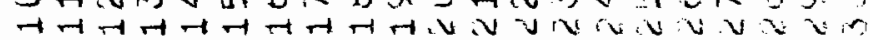




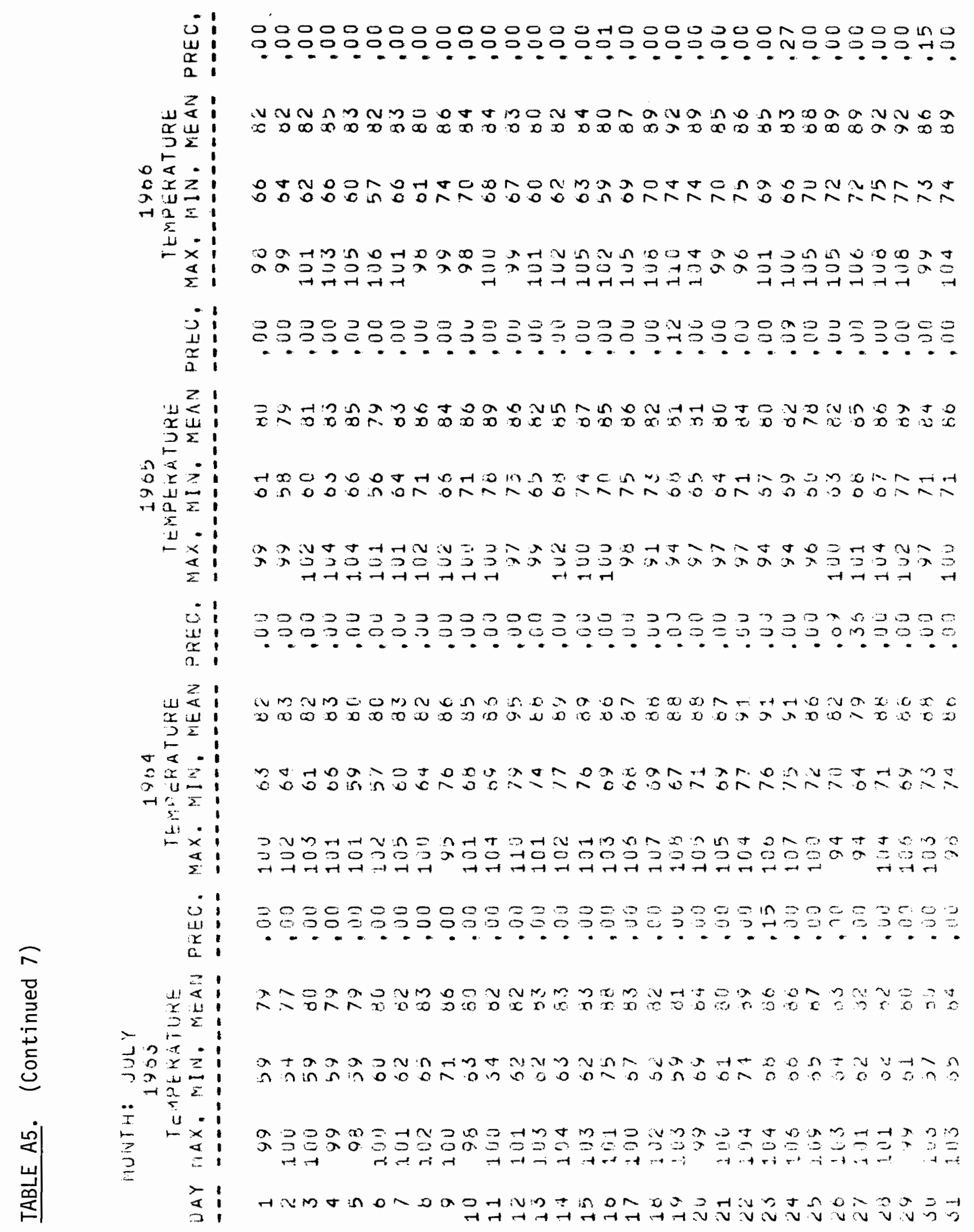




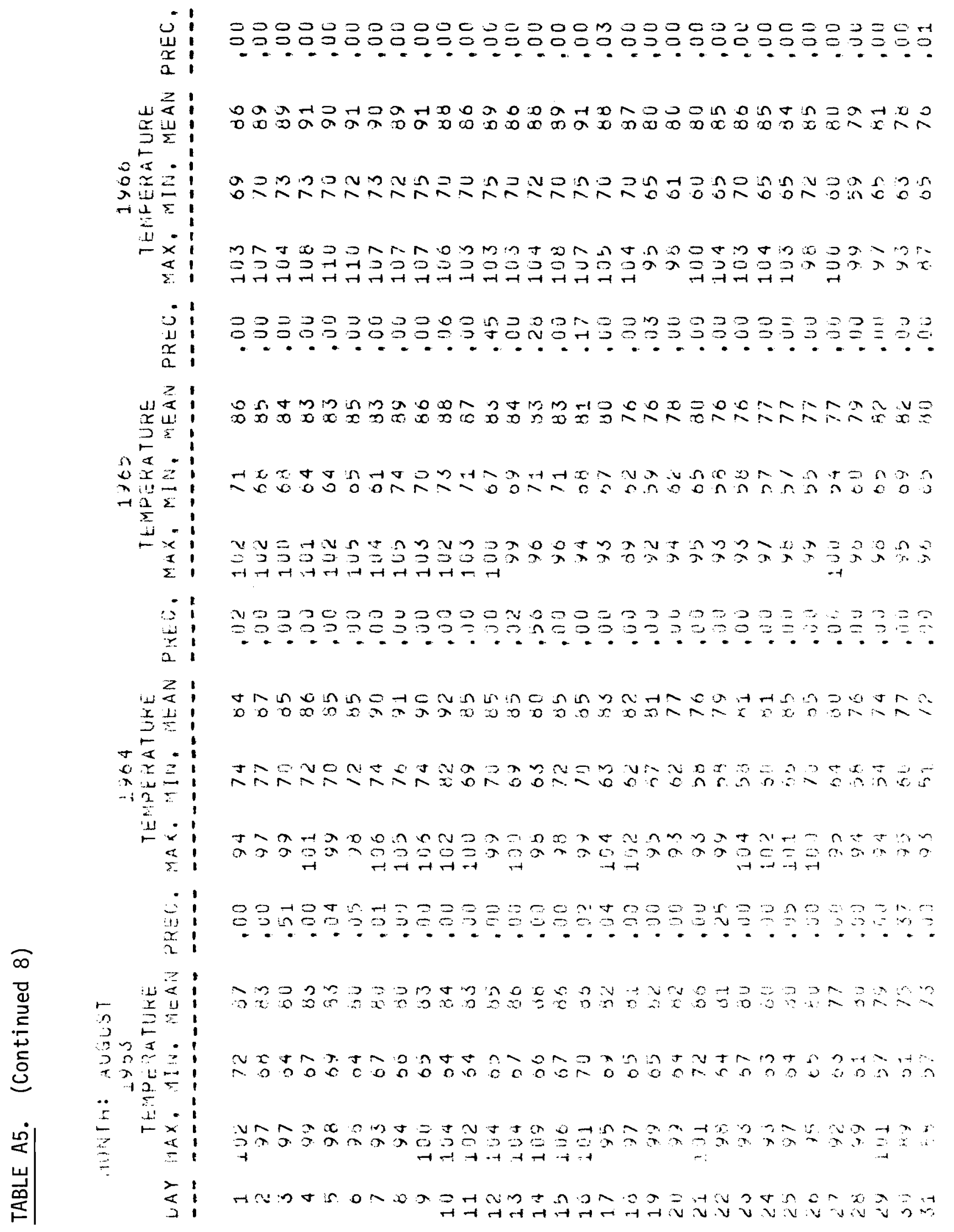


U:

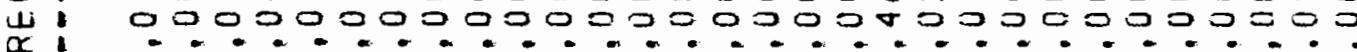

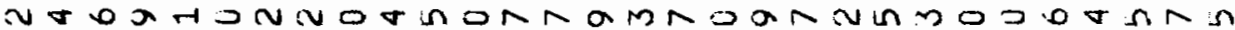
$\frac{2}{5}$ NAN

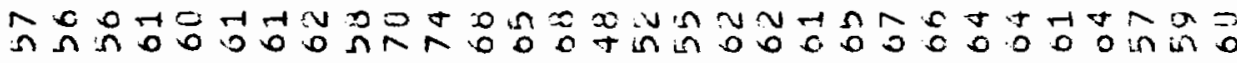

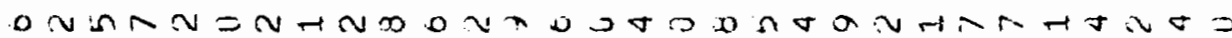

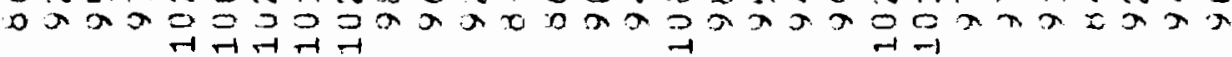

- 1

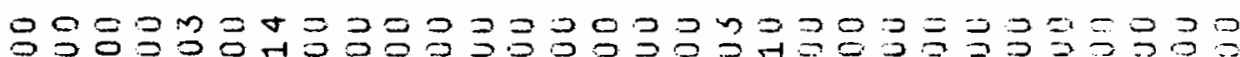

1

21

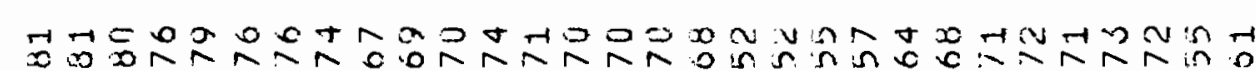

$\Upsilon \mathrm{w}$

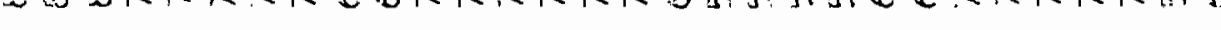

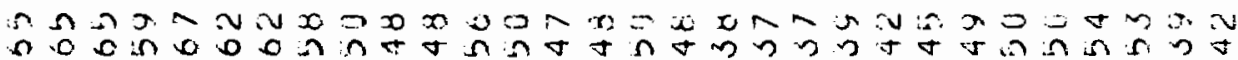

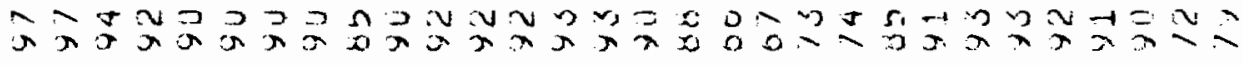

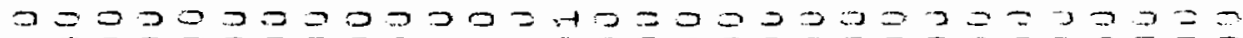

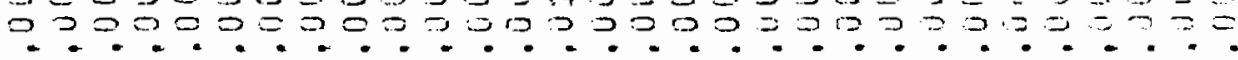

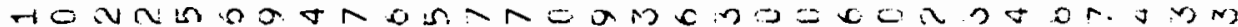

$x<1$

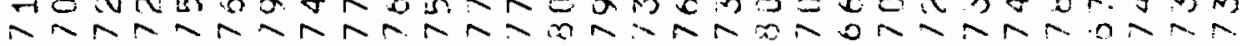

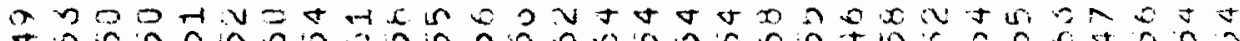
$r i a=$ s.

i.

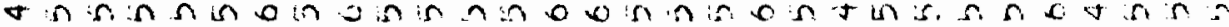

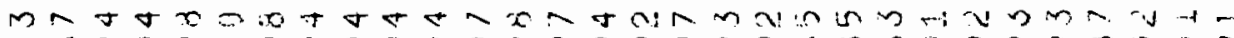

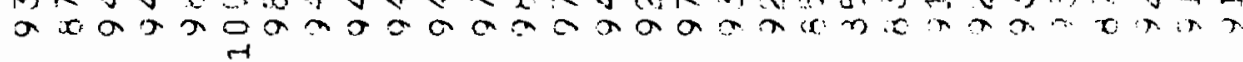

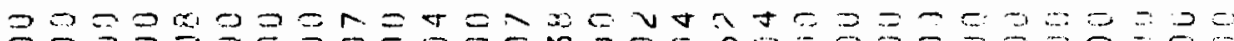

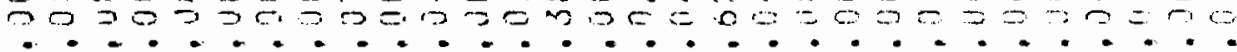

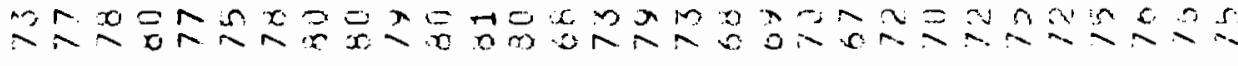

$1<1$

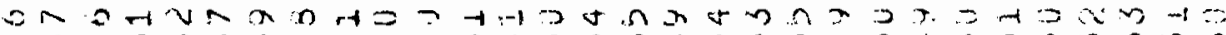

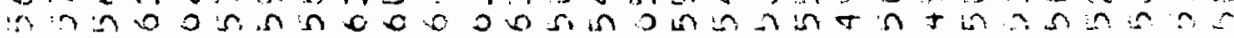

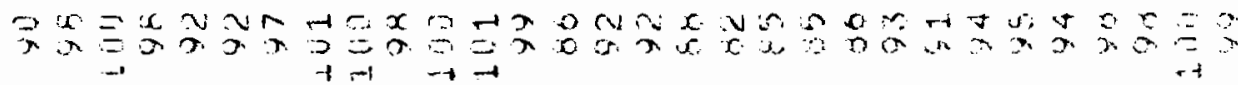

$>$

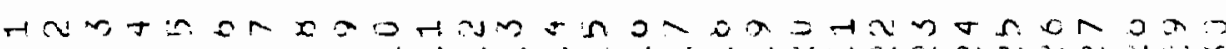

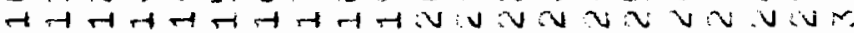


op 000000000R000000000000000000=c. 0000000000700000000000000000000

mata tht

in $\overline{1}$

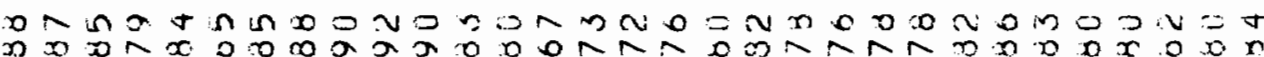

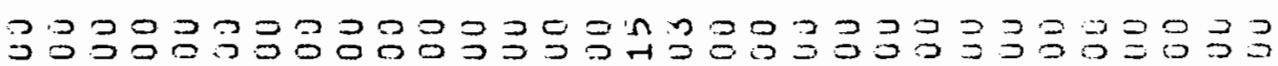

a.

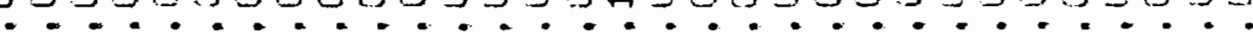

$\geq$

$11<$

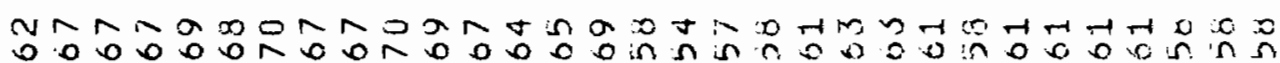

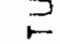

$\Omega \sim$

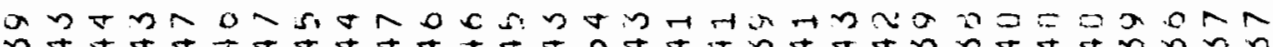
$\rightarrow$

I.

$+x$

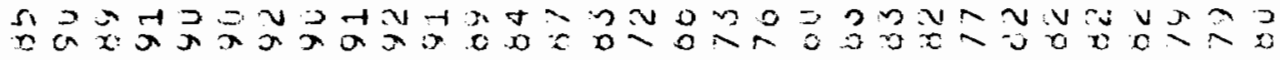

$\cdot 1$

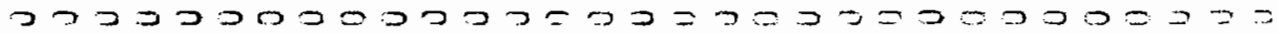

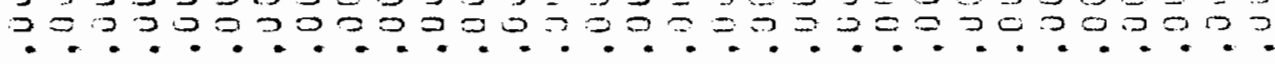

L $2 \geq 1$

$\frac{1}{2}$

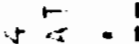
$0 x \geq$

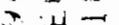

$-18$ $\Sigma$

1)

$\underset{x}{x}$

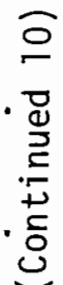

$-1$

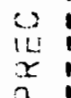

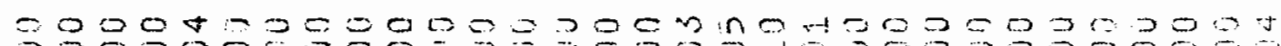

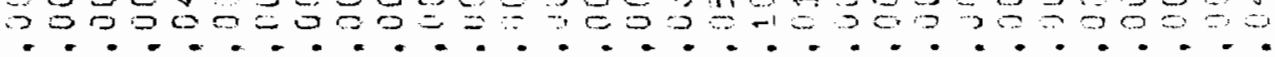

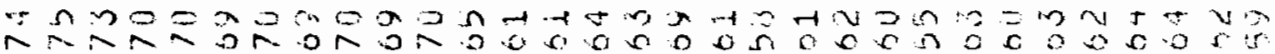
H. , $0 \geq$ $\rightarrow 2$. is $\geq 1$ i) $x$ כ ב $\div !$ :

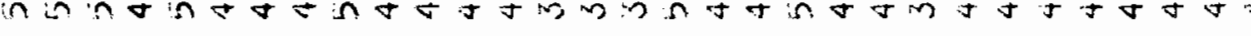

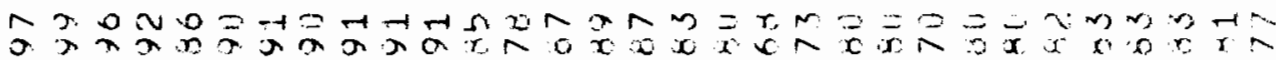

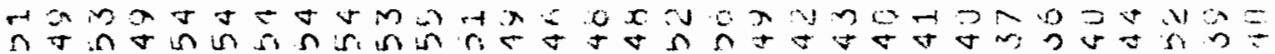
Hanthing a

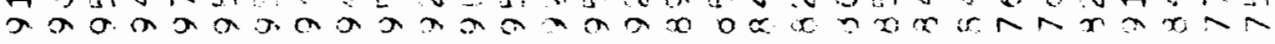
$\leq 1$ $\rightarrow$ ud 


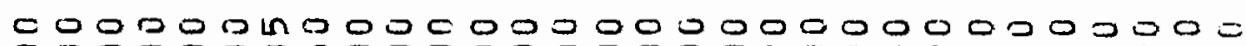
500000000000000000000000000000 崖:

* o o o on

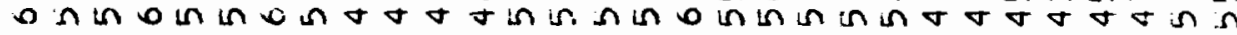

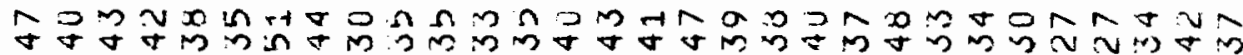

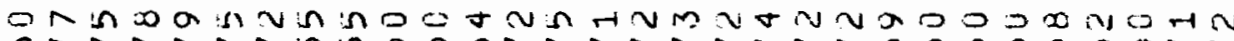
orrarkniningo on 년

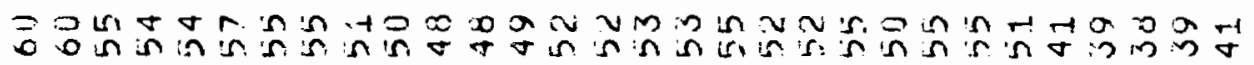

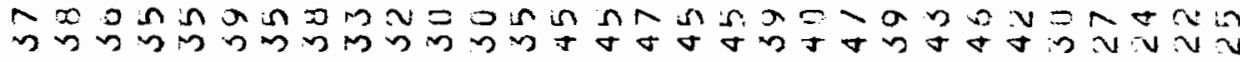

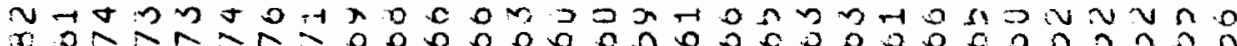

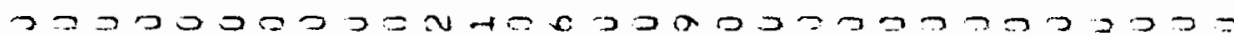

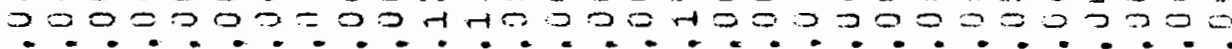
2!

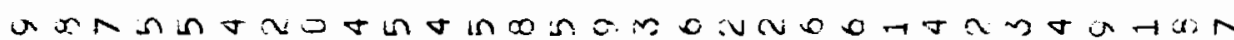

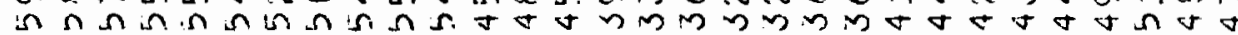

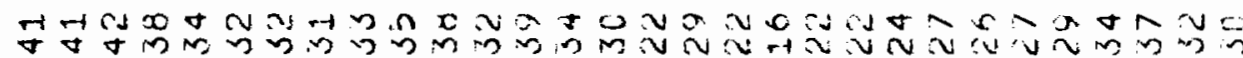

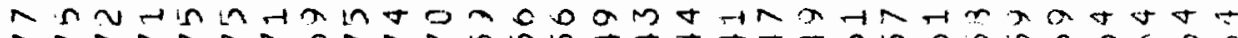

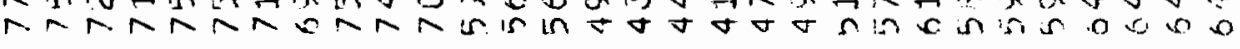

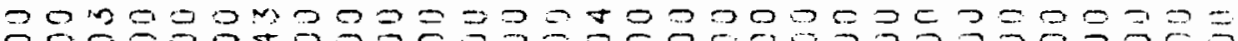

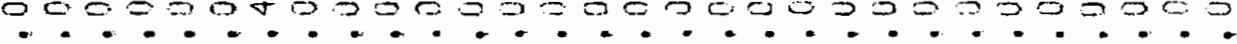

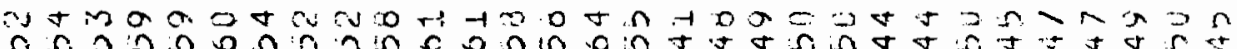

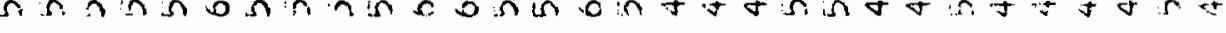




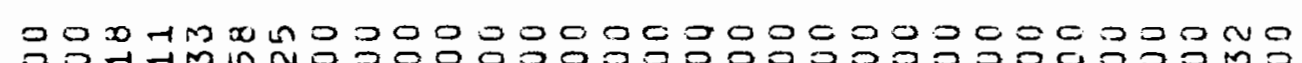

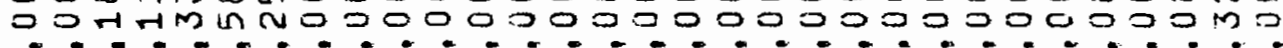

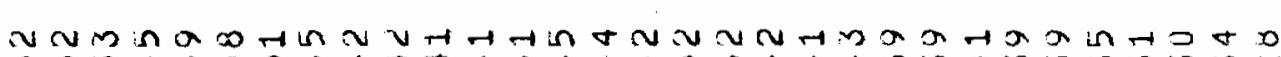
$x+\frac{1}{2}$

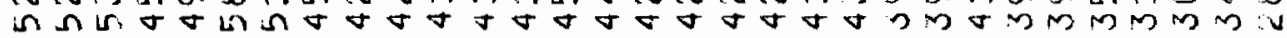

$0 \div$

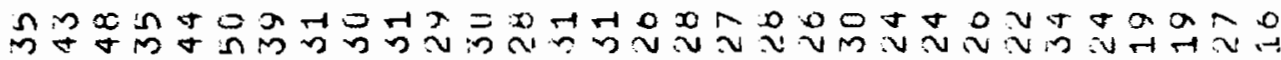

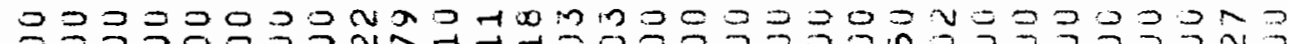

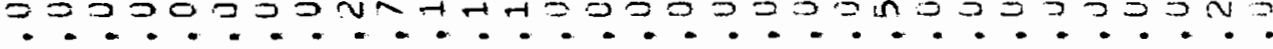

$\geq 1$

14⿻

ए

$\lim ^{2}$

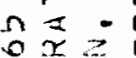

ग $15 \rightarrow$

$\rightarrow 2 \Sigma$ $\sum$

!U

$-\times 1$

$\therefore-$

I

$\underbrace{3}_{0}$

$x$

u: $\frac{2}{x}$

我 1

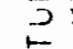

$\nabla$

$2 x \div i$

2 iu $=$

$\rightarrow+2$

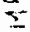

$\therefore$

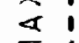

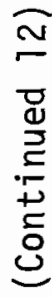

号

a

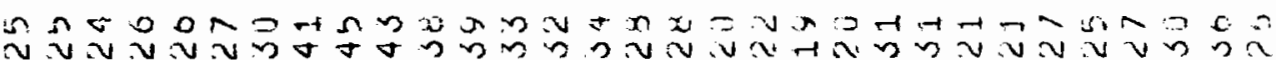

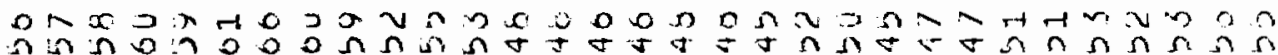

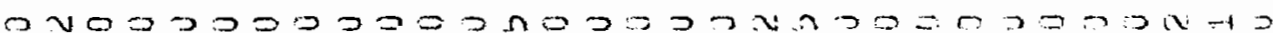

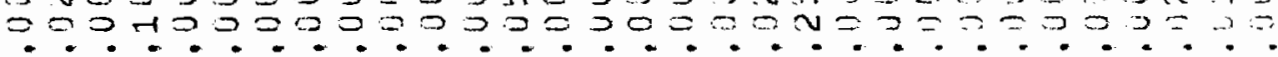

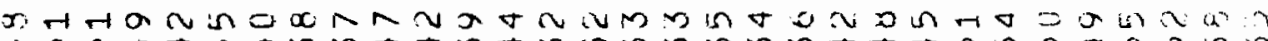

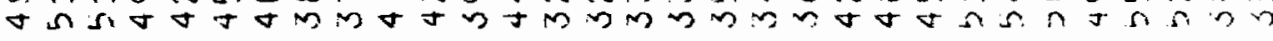

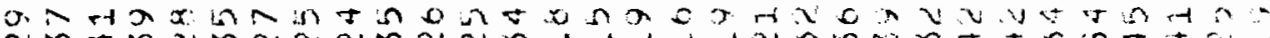

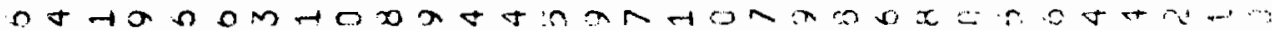

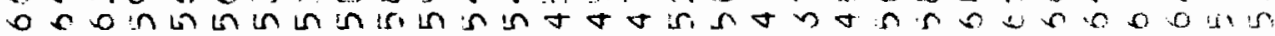

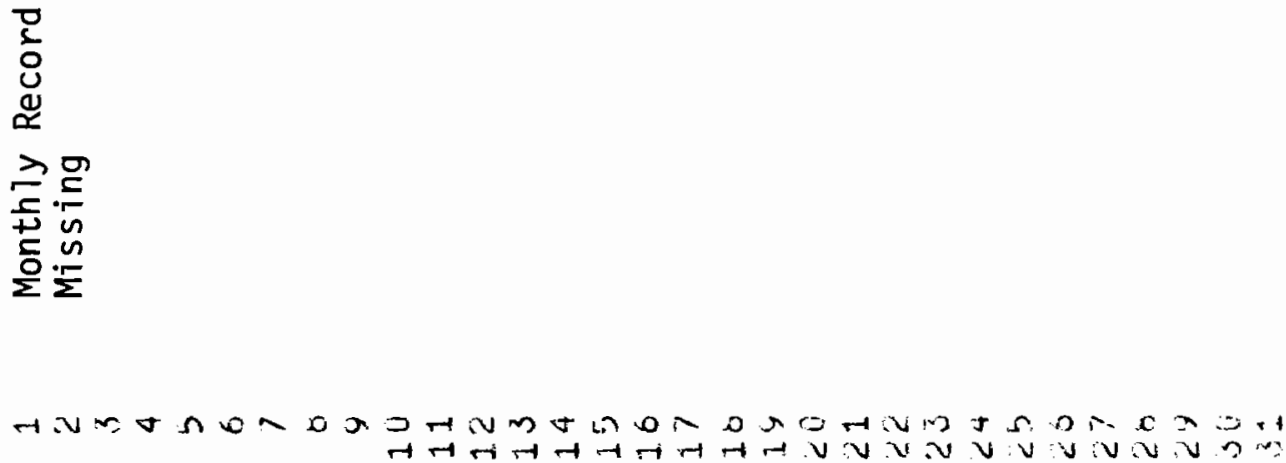




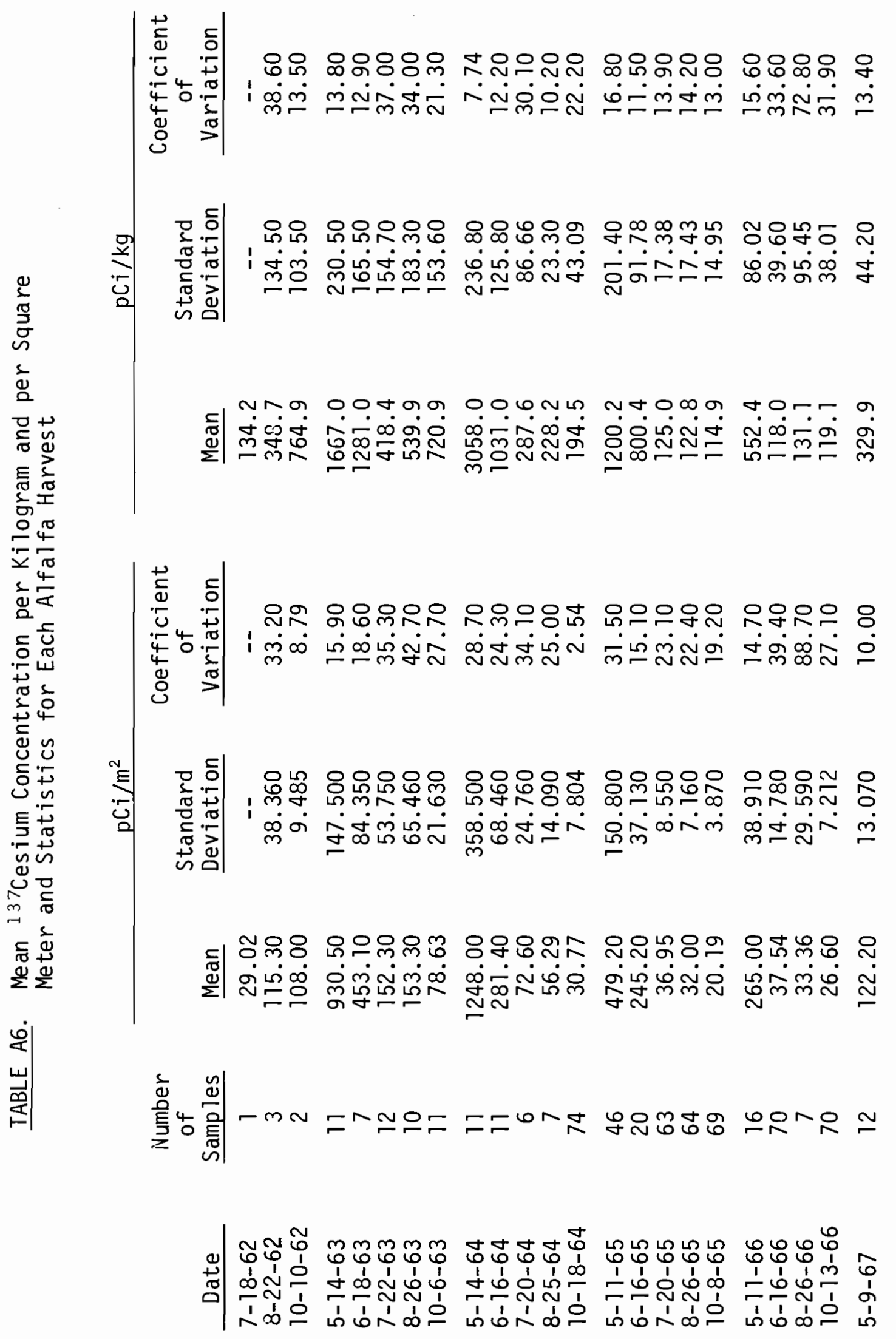




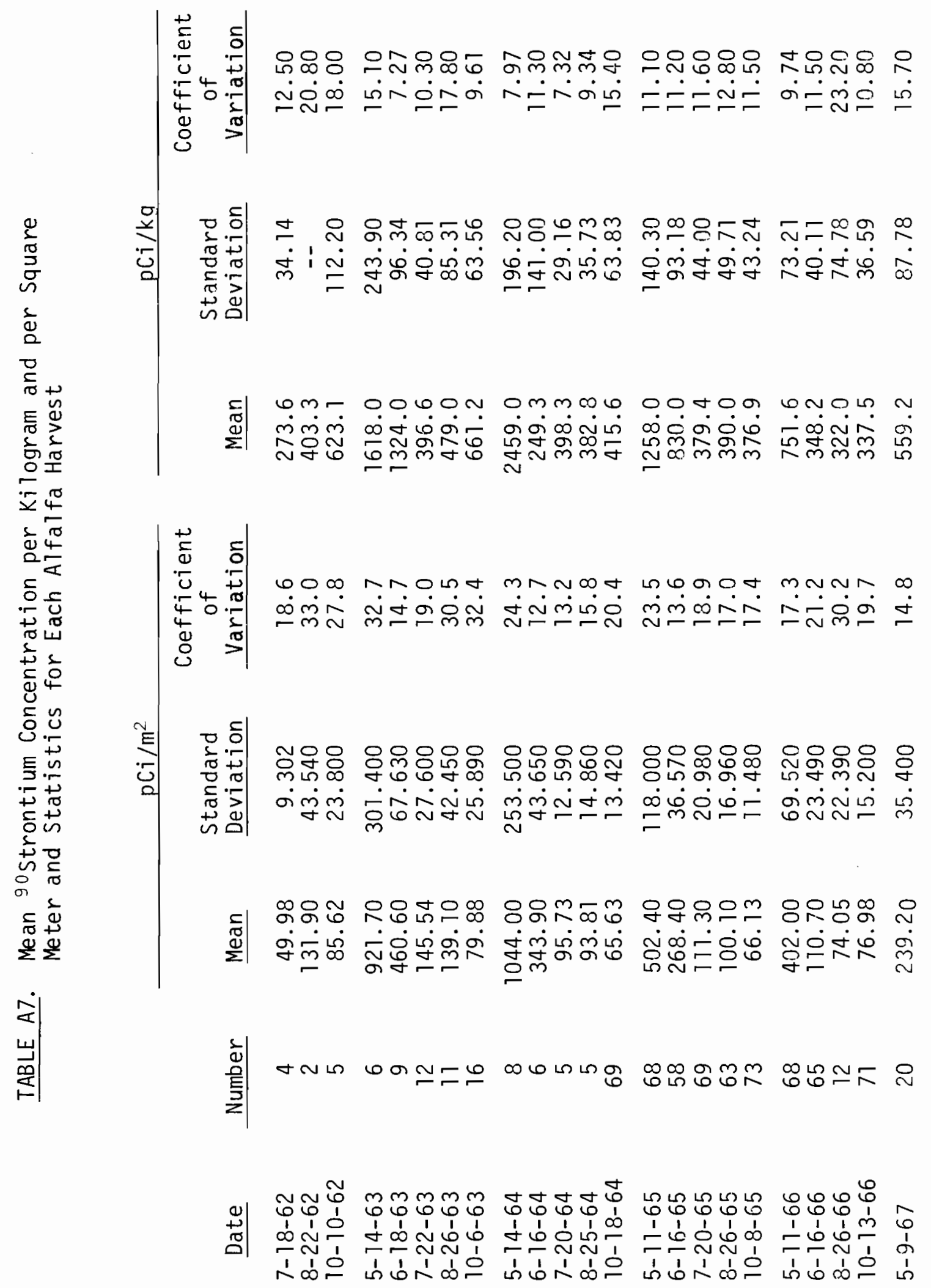




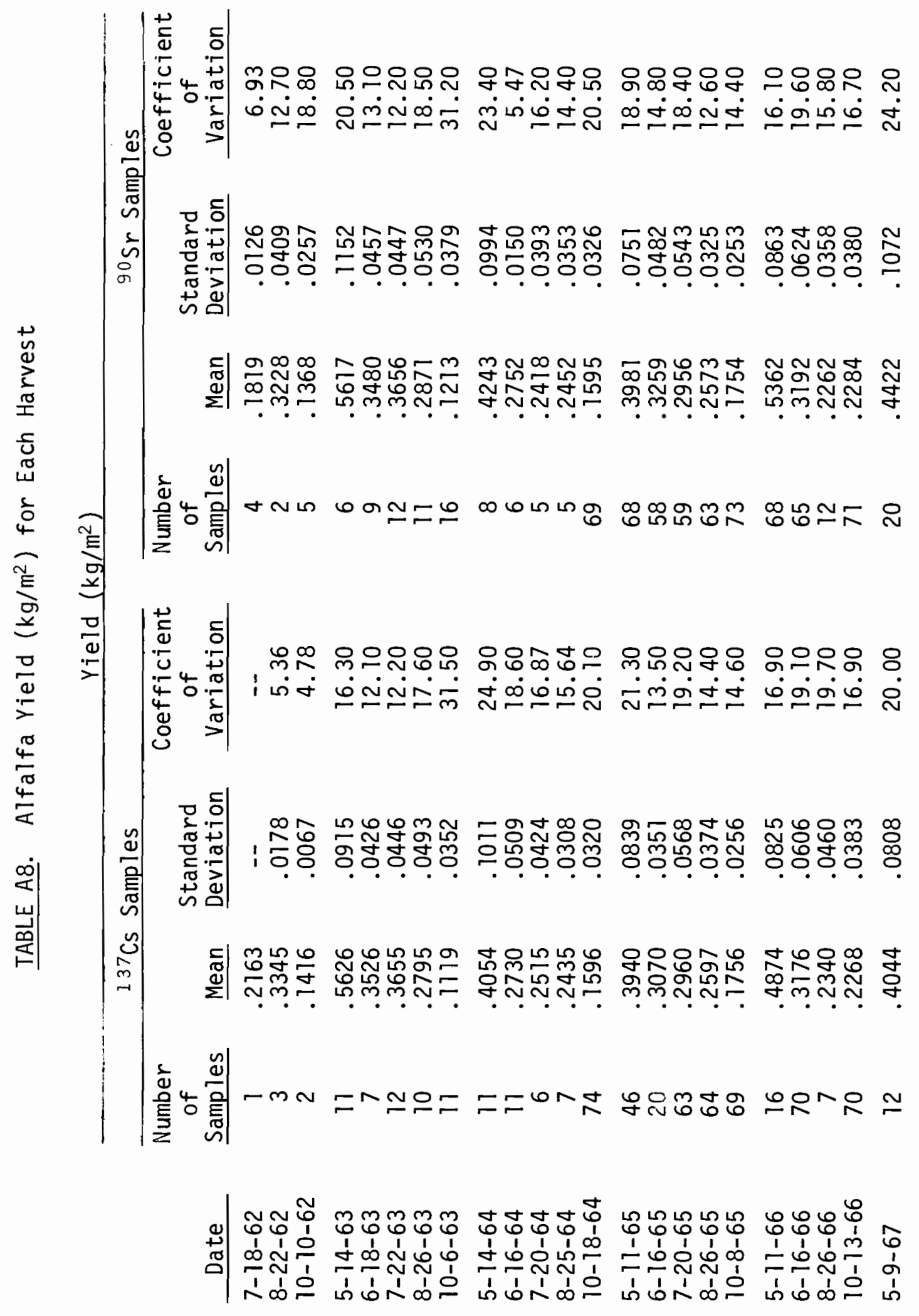


TABLE A9. CESIUM-137 IN IIIDIVIDUAL ALFALFA SAMPLË

\begin{tabular}{|c|c|c|c|c|}
\hline $\begin{array}{l}\text { SAMPLE } \\
\text { NUMEER }\end{array}$ & $\begin{array}{l}\text { FIELD } \\
\text { LCCATION }\end{array}$ & $\mathrm{FCl} / \mathrm{M} 2$ & $P C I / K G$ & $K G / M Z$ \\
\hline & SAN & ES CCLLECTED & $2 / 14 / 03$ & \\
\hline 1 & $N F$ & $1036.2 ?$ & 1560.010 & .5271 \\
\hline 2 & UF & 952.25 & 1822.00 & .3226 \\
\hline 3 & $S F$ & 884.50 & 1881.00 & .4703 \\
\hline 4 & $P_{1}$ & 843.09 & 1755.00 & .4594 \\
\hline$b$ & $\mathrm{~F} 2$ & $\operatorname{cs} 3.63$ & $175, .00$ & .3421 \\
\hline$t$ & 23 & 1047.31 & 1773.00 & .5707 \\
\hline 7 & $p 4$ & 935.09 & 1729.00 & .5408 \\
\hline$\dot{\phi}$ & 22 & $61+.11$ & $122 y .00$ & .4947 \\
\hline$y$ & $2 \varepsilon$ & 843.48 & 1373.00 & .0121 \\
\hline 10 & 29 & $1<02.30$ & $152 \leq .00$ & .7899 \\
\hline 11 & 30 & 422.20 & $147 \div .00$ & 6236 \\
\hline
\end{tabular}

SAMPLES COLLFCTED 6/13/03

$\begin{array}{lllll}1 & N F & 527.03 & 1281.00 & .4114 \\ 2 & C F & 511.22 & 1253.00 & .4032 \\ j & S F & 402.71 & 1203.00 & .3548 \\ 4 & F 1 & 555.45 & 1120.00 & .3510 \\ 5 & F 2 & 457.20 & 1407.00 & .3249 \\ 6 & F 3 & 521.09 & 1103.00 & .2811 \\ 7 & +4 & 556.04 & 1576.00 & .3528\end{array}$

SAMPLES CCLLECTEL $7 / 22.63$

\begin{tabular}{|c|c|c|c|c|}
\hline 1 & $N F$ & 105.17 & 354.30 & .2463 \\
\hline 2 & LF & 206.75 & 448.90 & .4006 \\
\hline 3 & $S F$ & $15 \%, 21$ & 450.50 & .3429 \\
\hline 4 & $P_{1}$ & 146.22 & 405.10 & .3509 \\
\hline$b$ & $P \bar{C}$ & 176.48 & 410,00 & .4004 \\
\hline 0 & $\mathrm{~F} 3$ & 122.71 & $3 \mathrm{sin} .30$ & .3227 \\
\hline 7 & $F_{4}$ & 143.28 & 400.10 & .3528 \\
\hline$\varepsilon$ & 96 & 109.83 & 297.00 & .5576 \\
\hline 9 & 97 & 293.84 & 860.30 & .3330 \\
\hline 16 & 99 & 118.90 & $3 \pm 3.10$ & .3740 \\
\hline 11 & 100 & 136.49 & 356.30 & .3031 \\
\hline 12 & \pm 02 & 110.05 & 205.301 & .3593 \\
\hline
\end{tabular}

SAMFLES COLLECTED $8 / 26 / 63$

$\begin{array}{lllll}1 & M F & 252.36 & 919.80 & .2744 \\ 6 & C F & 202.26 & 681.10 & .2470 \\ 3 & P 1 & 264.66 & 703.40 & .3703 \\ 4 & P 2 & 150.30 & 537.50 & .2790 \\ 5 & P 3 & 104.70 & 487.70 & .2147\end{array}$


TABLE A9. (Continued 2)

CESIUM-137 IN aLFALFA (CONT,

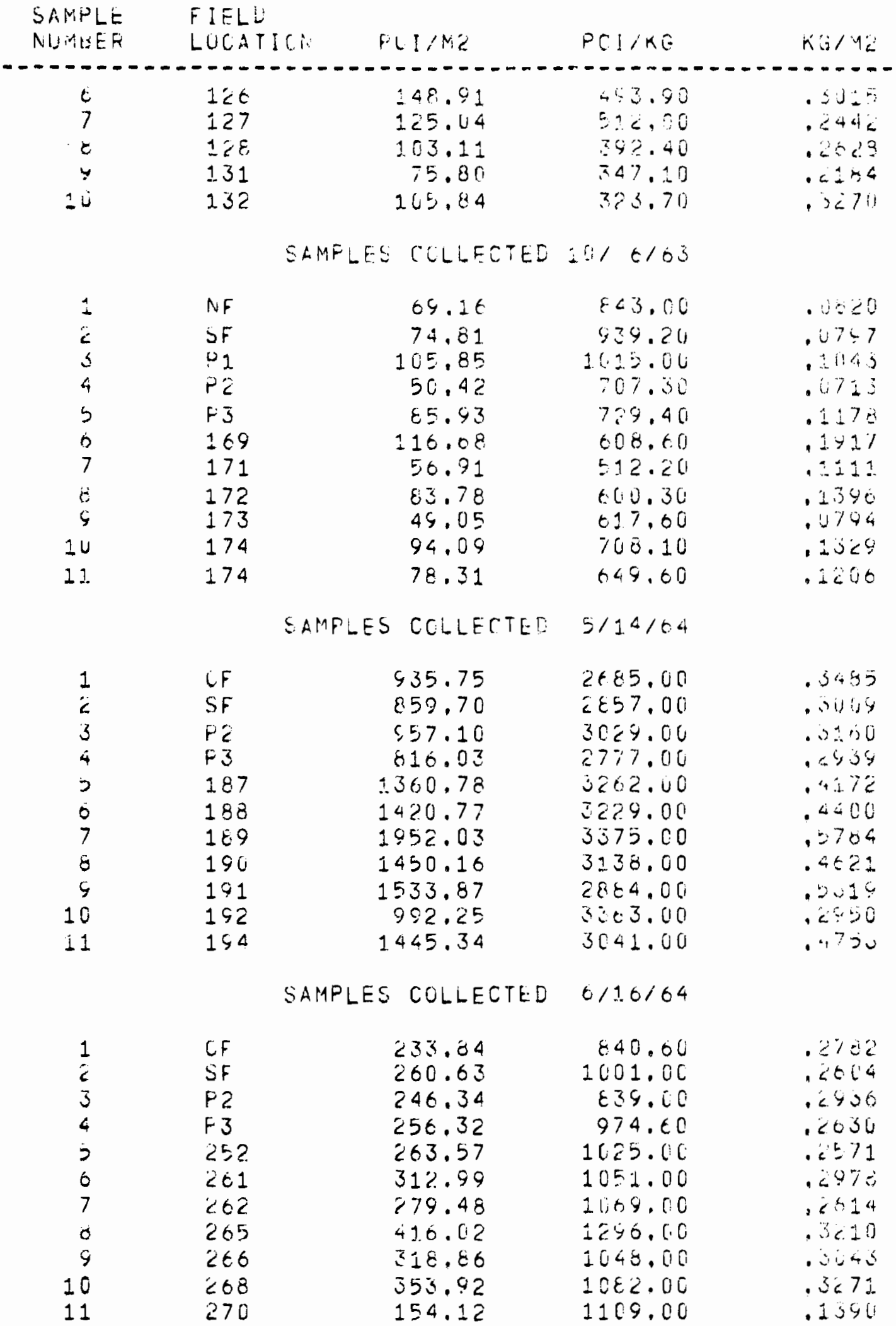


TABLE A9. (Continued 3)

CESIUM-137 IN ALFALFA (CONF,)

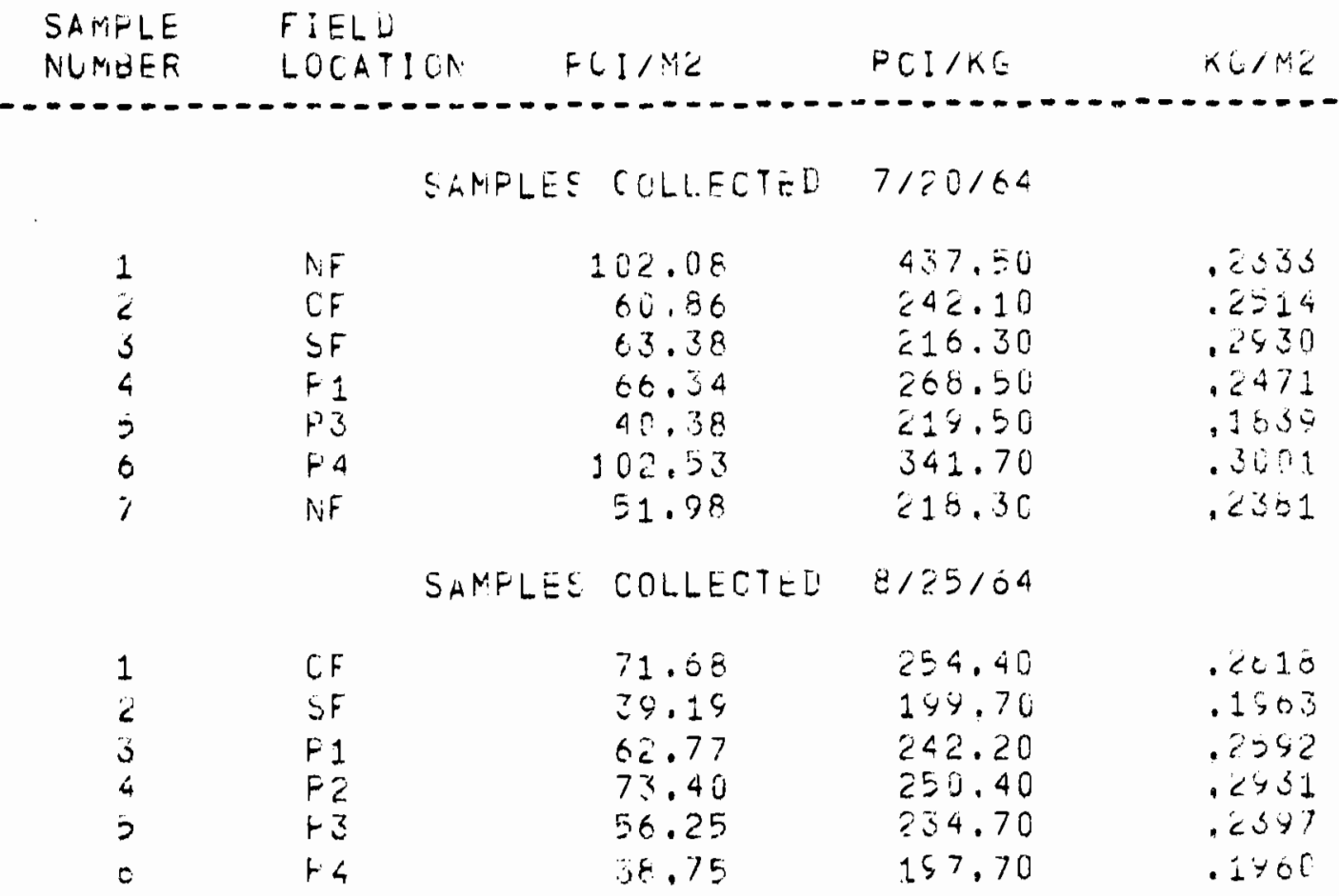

SAMPLES COLLECTED $10 / 13 / 64$

\begin{tabular}{|c|c|c|c|c|}
\hline 1 & $\mathrm{IE}$ & 30.09 & 2013.90 & .1490 \\
\hline $\bar{z}$ & $C F$ & $3 \pm .29$ & 234.00 & .1337 \\
\hline 3 & SF & 20.32 & 211.40 & $.1<45$ \\
\hline 6 & $F 1$ & 30.01 & 202.70 & .1075 \\
\hline 5 & $F^{\prime}$ & 29.44 & 230.00 & .1247 \\
\hline 0 & P3 & 34.46 & 227.20 & .1517 \\
\hline 7 & $F 4$ & $3[.17$ & $21 \mathrm{z} .50$ & $.14 \% 0$ \\
\hline 8 & 581 & 28.92 & 181.80 & .1591 \\
\hline 9 & 582 & 25,41 & 181.40 & $.14 i 6$ \\
\hline 10 & 583 & 30.01 & 243.40 & $.2<33$ \\
\hline 11 & 584 & 25.93 & 155.30 & .10 .70 \\
\hline 12 & 585 & 33.63 & 212.40 & .2583 \\
\hline 13 & 586 & 31.50 & 199.70 & .1578 \\
\hline 14 & 507 & 47.19 & 265.50 &, $\pm 77 \%$ \\
\hline 15 & 588 & 36.57 & 289.30 & .1664 \\
\hline 16 & 589 & $9.9 \mathrm{G}$ & 81.10 & .1232 \\
\hline 17 & 591 & 14.15 & 928.60 & .1100 \\
\hline is & 552 & 30.58 & 170.10 & .1798 \\
\hline 19 & 593 & 44.22 & 210.20 & .2104 \\
\hline 20 & 594 & 41.37 & 192.70 & .8147 \\
\hline 21 & 545 & 24.88 & 187.60 & .1326 \\
\hline 22 & 596 & 55.22 & $z \in 6.40$ & .2073 \\
\hline 83 & 357 & 40.37 & 190,50 & . 1119 \\
\hline 24 & 598 & $3(1.04$ & $1 \in 1.10$ & .1865 \\
\hline 25 & $=99$ & 30.74 & 191.10 & .1609 \\
\hline
\end{tabular}


TABLE A9. (Continued 4)

CESIUH-1Z; I1, LLFALFA (CONT, )

\begin{tabular}{|c|c|c|c|c|}
\hline $\begin{array}{l}\text { SAMPLE } \\
\text { NUMBER }\end{array}$ & $\begin{array}{l}\text { FIELL } \\
\text { LOCATION }\end{array}$ & $P C 1 / M 2$ & $P C I / K G$ & $K G / N 2$ \\
\hline$\cdots$ & $\ldots-\infty$ & $--\infty$ & $---\infty-\cdots$ &.------- \\
\hline 20 & 000 & 26.38 & 183.50 & .1448 \\
\hline 27 & 602 & 43.63 & 348.10 & .1253 \\
\hline ن & 603 & 25.44 & 227.70 & .1117 \\
\hline 2.9 & 604 & 20.03 & 153.40 & .1340 \\
\hline 30 & 005 & 20.30 & 182.50 & .1112 \\
\hline 31 & 006 & 23.35 & 210.60 & .1109 \\
\hline $3 \ddot{c}$ & 607 & 34.33 & 213.90 & 1005 \\
\hline 33 & 008 & 24.74 & 204.60 & $.1<04$ \\
\hline 34 & 609 & ¿5.36 & 178.40 & $.1=09$ \\
\hline 35 & 610 & 51.61 & 398.80 & .1294 \\
\hline 36 & 011 & 27.78 & 207.20 & .1341 \\
\hline 37 & 612 & 36.79 & 189.30 & .1943 \\
\hline 36 & $6 \pm 4$ & 23.39 & 176.00 & .1329 \\
\hline 34 & 015 & 24,49 & 201.90 & $.1<13$ \\
\hline 40 & 616 & 23.67 & 156.60 & .1512 \\
\hline 41 & 617 & 32.46 & 179.40 & .1810 \\
\hline 42 & 618 & 33.31 & 171.70 & .1940 \\
\hline 43 & 619 & 33.67 & 186.80 & .1002 \\
\hline 44 & 620 & 27.22 & 186.40 & .1400 \\
\hline 45 & 621 & 25.10 & 188.70 & .1330 \\
\hline 46 & 622 & 22.64 & $1 \in 4.50$ & .1377 \\
\hline 47 & 623 & 27.44 & 173.40 & .1502 \\
\hline 48 & 624 & 21.17 & $1 \in 7.00$ & $.126 t$ \\
\hline 49 & 625 & 47.94 & 214.60 & .2234 \\
\hline 50 & 026 & 32.51 & 193.50 & .1600 \\
\hline 51 & 627 & 30.67 & 199.40 & .1536 \\
\hline 52 & 628 & 25.84 & 167.50 & .1543 \\
\hline $\begin{array}{l}53 \\
54\end{array}$ & $\begin{array}{l}029 \\
030\end{array}$ & $\begin{array}{l}27.18 \\
28.77\end{array}$ & $\begin{array}{l}187.50 \\
155.30\end{array}$ & $\begin{array}{r}.1450 \\
.1853\end{array}$ \\
\hline 25 & 631 & 37.70 & $18 \Omega .00$ & .2071 \\
\hline $\begin{array}{l}56 \\
57\end{array}$ & $\begin{array}{l}632 \\
633\end{array}$ & $\begin{array}{l}27.44 \\
32.11\end{array}$ & 167.60 & $\begin{array}{r}1037 \\
.1799\end{array}$ \\
\hline 58 & 034 & 35.81 & $\begin{array}{l}178.50 \\
175.50\end{array}$ & .2040 \\
\hline $\begin{array}{l}59 \\
60\end{array}$ & $\begin{array}{l}635 \\
636\end{array}$ & $\begin{array}{l}39.75 \\
33.99\end{array}$ & $\begin{array}{l}164.70 \\
171.70\end{array}$ & $\begin{array}{l}.2414 \\
.1479\end{array}$ \\
\hline 61 & 637 & 29.90 & 152.80 & .1957 \\
\hline 62 & 038 & 28.48 & 189.00 & .1507 \\
\hline 03 & 639 & 30.60 & 172.20 & .1777 \\
\hline 04 & 640 & 36.90 & 180.20 & .2159 \\
\hline 65 & 641 & 28.52 & 144.60 & .1972 \\
\hline ot & 642 & 23.82 & 171.10 & .1392 \\
\hline 67 & 643 & 21.98 & 185.60 & .1184 \\
\hline 00 & 644 & 35.00 & 205.90 & .1845 \\
\hline 69 & 645 & 31,20 & 173.10 & $.180 \%$ \\
\hline 70 & 646 & 26.96 & 182.40 &, 1470 \\
\hline 71 & 647 & 34.89 & 195.50 & .1784 \\
\hline 72 & 648 & 29.06 & 178.80 & .1625 \\
\hline
\end{tabular}

A. 32 
TABLE A9. (Continued 5)

CESIUPI-137 IN ALFALFA (COT: T,

\begin{tabular}{|c|c|c|c|c|}
\hline $\begin{array}{l}\text { SAMFLE } \\
\text { NUMEER }\end{array}$ & $\begin{array}{l}\text { FIELD } \\
\text { LOCATI UN }\end{array}$ & $P C 1 / M 2$ & $P C I / K G$ & $K G / M z$ \\
\hline$\ldots-\cdots$ & $--\infty-\infty$ & $\ldots . .$. & 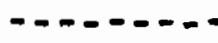 & $-\infty \ldots$ \\
\hline 73 & 649 & 23.73 & 221.70 & .1070 \\
\hline 74 & 650 & 33.14 & 192.70 & .1720 \\
\hline & SAY & $S$ COLLECTED & $5 / 11 / 65$ & \\
\hline 1 & $P: F$ & 546.62 & 1355.60 & .4034 \\
\hline 2 & $C F$ & 443.78 & 1341.00 & .3309 \\
\hline 3 & $C F$ & 342.16 & 1034.00 &, 3309 \\
\hline 4 & $S F$ & 539.53 & 1342.00 & .4017 \\
\hline 5 & $F_{1}$ & 588.96 & 1463.00 & .4026 \\
\hline 6 & $F 2$ & 633,20 & 1443.00 & .4358 \\
\hline 7 & $F \bar{S}$ & 503.52 & 806.70 & .6226 \\
\hline c & $F_{1}$ & 689.58 & 1384.06 & .4982 \\
\hline 9 & +2 & 486.90 & 1240.00 & .341 \\
\hline 10 & Fi3 & 352.66 & 235,60 & .4200 \\
\hline $1 i$ & $F 4$ & 708.91 & 1602,00 & .4425 \\
\hline 12 & Pb & 323.29 & 933,40 & .3464 \\
\hline 13 & $F 6$ & $533.5 \%$ & 1441,00 & .3703 \\
\hline 14 & PG & $450: 26$ & 1216.00 & .3703 \\
\hline 15 & 811 & 748.57 & 1410.00 & .5309 \\
\hline 16 & 820 & 912.92 & $154 \mathrm{~d}, 00$ &, 5857 \\
\hline 1.7 & 821 & 186.81 & 895.10 & .2087 \\
\hline 18 & 823 & 395.03 & .1137 .00 & .0474 \\
\hline 1. & 825 & 535.04 & $1 \widehat{c} 72,00$ & .4206 \\
\hline 0 & 832 & $=03.5 t$ & 1249.00 & .4032 \\
\hline 21 & 836 & 546.45 & 1393.00 & .3923 \\
\hline $2 \bar{C}$ & E3s & 620.74 & 1346.00 & $.461 \mathrm{C}$ \\
\hline 23 & 840 & 609.00 & 1340,00 & .4545 \\
\hline 24 & 841 & 600.66 & $131 c .00$ & .4578 \\
\hline 25 & 844 & 262.18 & 965.30 & .2716 \\
\hline$\angle 6$ & $\varepsilon 48$ & 318.42 & 1040.00 & $.30<2$ \\
\hline 27 & 849 & 317.77 & 973.00 & .3264 \\
\hline 20 & 850 & 349.37 & 1090.00 & .3205 \\
\hline 29 & 852 & 431.64 & 950.00 & .4544 \\
\hline 30 & 855 & 254.50 & 1029,06 & .2473 \\
\hline 31 & 856 & 396.37 & 975.50 & .4062 \\
\hline 32 & 861 & 485.01 & $109 y .00$ & .4413 \\
\hline 33 & 862 & 346.17 & 1.202 .00 & CE $\operatorname{cou}$ \\
\hline 34 & 064 & 548.86 & 1469,00 & $.373 \mathrm{t}$ \\
\hline 35 & 866 & 492.83 & 1176.00 & .4191 \\
\hline 36 & 867 & 452.81 & 1257,00 & .3602 \\
\hline 37 & 869 & 450.81 & 1152,00 & .3413 \\
\hline 36 & 873 & 324.42 & 961.90 & .3375 \\
\hline 39 & 875 & 322.03 & 973.10 & .3309 \\
\hline 40 & 876 & 6.47 .27 & 1334,00 & .4852 \\
\hline 41 & 879 & 373.89 & 1244.00 & .3006 \\
\hline $4 \hat{2}$ & 883 & 514.78 & 1206,00 & $4 \angle E E$ \\
\hline
\end{tabular}


TABLE A9. (Continued 8)

CESIUN-137 IN ALFALFA (COMT,)

SAMPLE FIELD

NUMBER LOCATION PLI/MZ FCI/KG KG/MZ

SAMPLES COLLECTER $8 / 26 / 65$

\begin{tabular}{|c|c|c|c|c|}
\hline 1 & lif & 33.80 & 137.80 & .2453 \\
\hline 2 & $C F$ & 43.52 & 141.10 & .3086 \\
\hline 3 & $S F$ & 36.21 & 133.80 &.$<707$ \\
\hline 4 & $F_{1}$ & 39.50 & 131.20 & .3010 \\
\hline 5 & $F \bar{z}$ & 44.04 & 151.30 & .2911 \\
\hline 0 & PS & 39.26 & 200.10 & .3820 \\
\hline 7 & PS & 34.23 & 121.90 & .2008 \\
\hline$\ddot{E}$ & $P 1$ & $46.8 \%$ & 154.50 & .3034 \\
\hline 4 & $P 2$ & 37.91 & 126.90 & .2988 \\
\hline 10 & F3 & 34.63 & 155.50 & .2227 \\
\hline 11 & H4 & 28.69 & $12 \angle .90$ & .233 \\
\hline 12 & P5 & 36.23 & 134.40 & .2690 \\
\hline 13 & P6 & 53.29 & 166.50 & .3200 \\
\hline 14 & 1661 & 19.75 & 86.10 & .2294 \\
\hline 15 & 1663 & 29.15 & 113.70 &, 2504 \\
\hline 16 & 1607 & 32.06 & $11 \hat{0} .30$ & .2893 \\
\hline 17 & 2669 & 28.37 & 104.40 & .2717 \\
\hline 18 & 1670 & 31.93 & 106.20 & .3007 \\
\hline 19 & 1672 & 23.03 & 78.20 & .2945 \\
\hline 20 & 1677 & 23.70 & 99.00 & .2380 \\
\hline 21 & 1678 & 29.65 & 117.90 & .2473 \\
\hline 22 & 1679 & 27.82 & 112.20 & .2479 \\
\hline 23 & 1680 & 24.07 & 103.70 & .2321 \\
\hline 24 & 1681 & 28.47 & 111.30 & .2350 \\
\hline 25 & 1682 & 19.78 & 90.90 & .2000 \\
\hline 20 & 1683 & 29.75 & 109.00 & .2729 \\
\hline$<7$ & 1685 & 26.59 & 131.00 & .2030 \\
\hline 28 & 1686 & 25.17 & 210.90 & .2204 \\
\hline 29 & 2087 & 28.00 & 109.70 & .2607 \\
\hline 30 & 1680 & 25.00 & 104.70 & .2397 \\
\hline 31 & 1689 & 24.05 & 113.60 & .2170 \\
\hline 32 & 1691 & 27.10 & 123.60 & .2192 \\
\hline 33 & 1692 & 23.60 & 98.90 & .2386 \\
\hline 34 & 1690 & 36.35 & 130,90 & .2617 \\
\hline 35 & 1694 & 34.93 & 125.90 & .2775 \\
\hline je & 1690 & 27.74 & 102.10 & .2717 \\
\hline 37 & 1697 & 26.11 & 130.60 & .1911 \\
\hline 38 & 1688 & 28.51 & 128.70 & .2215 \\
\hline s\$ & 1699 & 27.09 & .207 .40 & .2522 \\
\hline 40 & 1.700 & 29.76 & 123,20 & .2476 \\
\hline 41 & 1701 & 29.29 & 122.40 & .2595 \\
\hline $4 a$ & 1.702 & 34.51 & 130.70 & .2641 \\
\hline 43 & 1705 & 29.62 & 118.50 & .2500 \\
\hline 44 & 2.704 & 36.017 & 136.00 & .2647 \\
\hline
\end{tabular}


TABLE A9. (Continued 9)

CESIUM-137 IN ALFALFA (CON9,)

\begin{tabular}{|c|c|c|c|c|}
\hline $\begin{array}{l}\text { SAMPLE } \\
\text { NUMEER }\end{array}$ & $\begin{array}{l}\text { FIELD } \\
\text { LOCAT1ON }\end{array}$ & $P C I / M 2$ & $P C I / K G$ & $K G / M Z$ \\
\hline$-m---.=$ & $--m-m--$ & $\cdots----\infty$ & $----\infty$ & $---\infty--$ \\
\hline $4 b$ & 1706 & 35.21 & 117.40 & .3000 \\
\hline 40 & 1707 & 34.68 & 132. บ & .2628 \\
\hline 47 & I7L & 26.11 & $14 \pm .60$ & .1905 \\
\hline 48 & $\therefore 769$ & 30.13 & 117.90 & .2556 \\
\hline 49 & 1711 & 39.58 & 135.70 & .2095 \\
\hline 50 & $171 \%$ & 29.50 & 129.20 & .2283 \\
\hline 51 & 1713 & 28.79 & 121.20 & .2372 \\
\hline 52 & 1714 & 38.03 & 152.80 & .2341 \\
\hline 53 & 1715 & 39.20 & 146.50 & .2662 \\
\hline 54 & 1710 & 41.19 & 134.90 & .3053 \\
\hline 55 & 1717 & 34.56 & 125.60 & .2752 \\
\hline 56 & 1718 & 37.11 & 122,10 & .3039 \\
\hline 57 & 1719 & 27.23 & 121.90 & .2234 \\
\hline 56 & $172 \mathrm{c}$ & 24,35 & 106.40 & .2260 \\
\hline 59 & $17 \hat{c}$ & 51.78 & 122.70 & .26112 \\
\hline 00 & 1724 & 28.75 & $115: 30$ & .2581 \\
\hline 01 & 1725 & 47.96 & 150.20 & .3193 \\
\hline E\% & 1720 & 22.41 & 1.03 .30 & .2110 \\
\hline 03 & 1727 & 22.01 & 114.30 & .1978 \\
\hline 64 & 1726 & 47.11 & 143.70 & .3278 \\
\hline
\end{tabular}

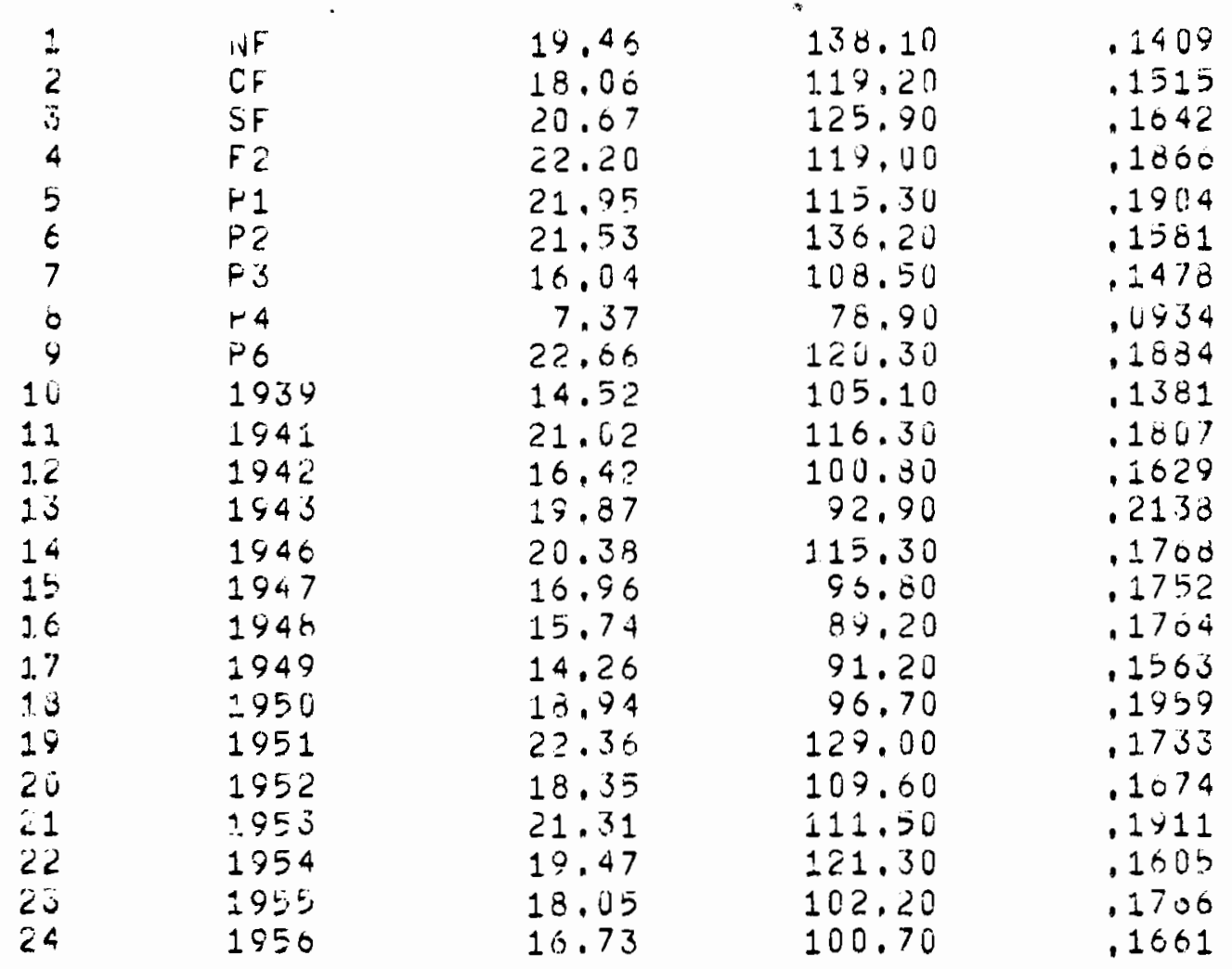


TABLE A9. (Continued 10)

CESIUM-137 IN ALFALFA (CONT,)

\begin{tabular}{|c|c|c|c|c|}
\hline $\begin{array}{l}\text { SAMFLE } \\
\text { NUNEEF }\end{array}$ & $\begin{array}{l}\text { FIELD } \\
\text { LDCAT ION }\end{array}$ & $P 0 I / M 2$ & $P C I / K G$ & $K 0 / 12$ \\
\hline$---m-1$ & $-\infty-\infty-\infty$ & $----\cdots$ & $m--\cdots-\cdots$ & $---\infty$ \\
\hline 25 & 1957 & 21.71 & 111.30 & .1969 \\
\hline 26 & 1950 & $24.0 ?$ & 113.40 & .2171 \\
\hline 27 & $195 \%$ & $13 .+0$ & 102.20 & .1000 \\
\hline 26 & 1961 & 16.27 & 93.80 & .1047 \\
\hline 29 & 1902 & 16.32 & 132,20 & .1234 \\
\hline 30 & 1964 & 16.22 & 95.80 & .1684 \\
\hline 31 & 1965 & $14.3=$ & 93.20 & .1531 \\
\hline 32 & 1967 & $13.1 \mathrm{j}$ & 108.80 & .1504 \\
\hline 33 & 1963 & 17.03 & 111.40 & .1528 \\
\hline .34 & 1971 & 21.70 & 118.00 & .1847 \\
\hline 35 & 1972 & 15,48 & 98.00 & .1280 \\
\hline 30 & 1973 & 22.34 & 117.70 & .1898 \\
\hline 57 & 1974 & 13.50 & 110.10 & .1097 \\
\hline 38 & 1975 & 21.03 & 113.90 & .1769 \\
\hline 35 & 1976 & 24.45 & 130.20 & .1795 \\
\hline 40 & 1977 & 16.05 & 103.60 & .1549 \\
\hline 41 & 1978 & 19.70 & 122.00 & .1022 \\
\hline 42 & 1979 & 20.18 & 126.10 &, $160 \bar{U}$ \\
\hline 43 & 1980 & 20.37 & 124.40 & .1 .637 \\
\hline 44 & 1961 & 23.83 & $12 \mathrm{i} .80$ & .1 .457 \\
\hline 45 & 1982 & 26.51 & 142.20 & .1865 \\
\hline 46 & 1983 & 17.75 & 115.80 & .1533 \\
\hline 47 & 1984 & 23.63 & 154.10 & .1533 \\
\hline $4 n$ & 1985 & 17.09 & 121.30 & .1617 \\
\hline 49 & 1980 & 18.32 & $11 \equiv .20$ & .1564 \\
\hline 50 & 1987 & 25.52 & 140.20 & .1620 \\
\hline 3.1 & 1908 & 24.44 & 120.40 & $.203 j$ \\
\hline 52 & 1989 & 14.75 & 78.90 & .1869 \\
\hline 33 & 1997 & 22.64 & 116.30 & .1947 \\
\hline 54 & 1491 & 29.48 & $11 \supset .60$ & .2550 \\
\hline 5 & isy2 & 25.29 & 112.40 & . 2250 \\
\hline in & 1993 & 27.68 & 137.10 & .2019 \\
\hline 27 & 1994 & 23.15 & 118.50 & .1952 \\
\hline 50 & 1990 & 24.70 & 110.00 & .2060 \\
\hline $5 y$ & 1990 & 23.86 & 125,60 & .1885 \\
\hline ou & 1997 & 17.10 & 90.50 & .1790 \\
\hline $0 I$ & $199 ;$ & 24.43 & 125.70 & $: 1920$ \\
\hline 62 & 2000 & 20.52 & 119.40 & .1719 \\
\hline 6.3 & $<001$ & 25.00 & 123.90 & $.2 \cup 13$ \\
\hline 64 & 2002 & 16.84 & 117,60 & .1432 \\
\hline 63 & 2003 & 24.87 & 139.70 & .1782 \\
\hline en & 2005 & 24.52 & 110.40 & .2231 \\
\hline 67 & 2006 & 17.75 & 131.00 & .1355 \\
\hline $0 n$ & 2007 & 19.52 & 114,40 & .1707 \\
\hline 69 & 2008 & 23.71 & 109.10 & .2173 \\
\hline
\end{tabular}


TABLE A9. (Continued 11)

CESIUM-137 IN ALFALFA (CONT,)

\begin{tabular}{|c|c|c|c|c|}
\hline $\begin{array}{l}\text { SAIAFLE } \\
\text { INUMEER }\end{array}$ & $\begin{array}{l}\text { FIELD } \\
\text { LCCAT!ON }\end{array}$ & $P C I / M 2$ & $P C ! / K G$ & $K G / M 2$ \\
\hline & SAP & SS COLLECTED & $2 / 11 / 66$ & \\
\hline 1 & NF & 249.25 & 352.00 & .4209 \\
\hline$<$ & CF & 337.91 & 643.00 & .5255 \\
\hline 3 & $F_{1}$ & 224.21 & 575.40 & .3897 \\
\hline 4 & $F^{2}$ & $? 21.65$ & 563.50 & .3599 \\
\hline 2 & is & 20.3 .02 & 520.70 & .3899 \\
\hline 3 & +1 & 233.41 & 664.30 & .4492 \\
\hline 7 & $P \hat{z}$ & 285.01 & 037.20 & .4489 \\
\hline$\dot{0}$ & $\mathrm{P3}$ & 257.98 & 006.60 & .4253 \\
\hline$\exists$ & $P 4$ & 278.00 & 001.90 & .4632 \\
\hline 11) & 15 & $298.2 ?$ & 597.40 & .4992 \\
\hline 11 & $P 6$ & 319.81 & 605.30 & $.5<19$ \\
\hline ic & 2324 & 277.47 & 453.40 & $: 0120$ \\
\hline 13 & $\ddot{3} 340$ & 232.36 & 559.40 & .4154 \\
\hline 14 & 2351 & 245.66 & 413.70 & .5938 \\
\hline 15 & 2360 & 222.74 & 362.90 & .6138 \\
\hline 10 & 2385 & 286.84 & 475.30 & .5035 \\
\hline
\end{tabular}

$$
\text { EAMFLED COLEECTES, 0/10/5S }
$$

\begin{tabular}{|c|c|c|c|c|}
\hline 1 & NF & 33.81 & 107.10 & .3344 \\
\hline 2 & $C F$ & 32.67 & 101.50 & .3218 \\
\hline 3 & $S F$ & 33.81 & 90.70 & .3425 \\
\hline 4 & $F \perp$ & 29.69 & 112.70 & .2535 \\
\hline 3 & $\mathrm{~F} 2$ & 28.44 & 93,00 & .3050 \\
\hline 6 & PS & 30.24 & 92.50 & $.4 \geq 42$ \\
\hline 7 & $P_{1}$ & $24.9 j$ & 101.70 & .2449 \\
\hline y & $F 2$ & 31,60 & 100.30 & .2973 \\
\hline 9 & P3 & 36.07 & 108.40 & .3330 \\
\hline 10 & +4 & 22.83 & 80.00 & .2536 \\
\hline 11 & PS & 34.30 & 109.00 & .3147 \\
\hline 12 & 16 & 21.16 & 91.90 & .2302 \\
\hline 13 & 2000 & 30.87 & 113.70 & .2715 \\
\hline 24 & 2601 & 30.12 & 110.30 & .2540 \\
\hline $\begin{array}{l}15 \\
16\end{array}$ & $\begin{array}{l}2003 \\
2604\end{array}$ & $\begin{array}{l}31.2 \% \\
23\end{array}$ & 110.60 & $\begin{array}{r}.2532 \\
.1945\end{array}$ \\
\hline 17 & 2605 & 38.12 & 114.40 & .3332 \\
\hline 28 & 2606 & 22.53 & 128.30 & .1755 \\
\hline 19 & $260 \%$ & 32.58 & $\$ 17.00$ & .2784 \\
\hline 20 & 2608 & 43.43 & 142.80 & .3045 \\
\hline a 1 & 2609 & 18.14 & 95.10 & .1887 \\
\hline 22 & $2611 !$ & 25.29 & 123.90 & .2042 \\
\hline 23 & 2612 & 27.80 & 107.70 & .2531 \\
\hline 24 & 261.3 & 29.05 & 120.30 & .2416 \\
\hline 25 & 2014 & 35.15 & 110.00 & .2979 \\
\hline
\end{tabular}


TABLE A9. (Continued 12)

CESIU:1-137 IN ALFALFA (CONT,)

\begin{tabular}{|c|c|c|c|c|}
\hline $\begin{array}{l}\text { SAMFLE } \\
\text { NUMEER }\end{array}$ & $\begin{array}{l}\text { FIELU } \\
\text { LOCATION }\end{array}$ & $P C ! / M 2$ & $P C I / K G$ & $K \bar{G} / M Z$ \\
\hline$-m-\ldots m$ & $-----n$ & $---\infty-\infty$ & $--\infty-\infty-\infty$ & $\ldots--\infty$ \\
\hline 26 & 2617 & 34.66 & 114.00 & .3025 \\
\hline 27 & 201 i & 33.14 & $\$ 06.70$ & .3100 \\
\hline 28 & 2019 & 37.24 & 125.70 & .2962 \\
\hline 29 & 2626 & $3 y .51$ & 125.30 & .3153 \\
\hline su & 2621 & 33.36 & 121.90 & .2735 \\
\hline 31 & 2622 & $35.6 ?$ & 132.80 & .2033 \\
\hline $3 k$ & $\angle 623$ & 37.60 & 123.90 & .5056 \\
\hline 23 & 2624 & 30.33 & 127.10 & .2814 \\
\hline 34 & 2525 & 40.07 & 123.40 & .3296 \\
\hline 35 & 2627 & 39.03 & 121.00 & .3210 \\
\hline 36 & 6525 & 27.91 & 96.70 & .2380 \\
\hline 37 & 2023 & 34.43 & 102.00 & .3375 \\
\hline 39 & 263. & 31.37 & 89.00 & .5523 \\
\hline 39 & 2031 & 41.85 & 102,00 & .4103 \\
\hline 611 & 2033 & 31.01 & 94.50 & .3343 \\
\hline $4 \pm$ & $\begin{array}{l}2634 \\
2635\end{array}$ & $\begin{array}{l}36.74 \\
21.37\end{array}$ & $\begin{array}{r}100.20 \\
90.20\end{array}$ & $\begin{array}{r}.3667 \\
.2436\end{array}$ \\
\hline 4.3 & $203 \%$ & $29.2 ?$ & 103.40 & .2826 \\
\hline 44 & 2638 & 29.05 & $\$ 5.40$ & 3045 \\
\hline 45 & 2039 & 32.20 & 93.20 & .3455 \\
\hline $4 c$ & 2640 & 43.50 & 101.20 & 4298 \\
\hline 47 & 2641 & 32.53 & 96.13 & .5385 \\
\hline 48 & 2642 & 34.98 & 108.70 & 3218 \\
\hline 49 & 2644 & 36.02 & 115.70 & .3113 \\
\hline 50 & 2045 & 40.98 & 113,50 & .3011 \\
\hline 51 & 2647 & 45.89 & 111.00 & .4112 \\
\hline .22 & 2648 & 43.13 & 119.90 & .3001 \\
\hline 53 & 2649 & 49.12 & 123.70 & 3971 \\
\hline 54 & 2550 & 31.95 & 106.00 & .3014 \\
\hline 55 & 2651 & 32.42 & 93.80 & .3456 \\
\hline 56 & 2652 & 42.69 & 114.30 & .3735 \\
\hline $5 ?$ & 2054 & 42,41 & 118.00 & .3294 \\
\hline ع ל כi & 2653 & 32.63 & 100,60 & .3244 \\
\hline 5 & $<656$ & 44.60 & 109.80 & .4062 \\
\hline ou & 2657 & 40.40 & 110.60 & .3553 \\
\hline 61 & $265 y$ & 43.63 & 113.90 & .3931 \\
\hline+2 & 2060 & 50.44 & 110.20 & .4755 \\
\hline 0.3 & 2001 & 32.57 & 112.30 & .2855 \\
\hline 64 & 266.3 & 127.29 & 353.30 & .3236 \\
\hline 65 & 2664 & 42.77 & 123.20 & .3472 \\
\hline 0.6 & 2665 & 52,28 & 140.00 & .3581 \\
\hline 67 & 2665 & 33.33 & 117.90 & .2327 \\
\hline $6 y$ & $26 \in 7$ & 58.87 & \pm 23.90 & .4752 \\
\hline 63 & 26.60 & 68.31 & 212.10 & .3221 \\
\hline 74 & 2509 & 75.45 & 214.90 & .3581 \\
\hline
\end{tabular}


TABLE A9. (Continued 13)

CESIUIT-137 IN ALFALFA (CONT,)

SAMPLE FIELU

NUMEEF LOCATION PCI/M2 PCI/KG KG/M2

SAMOLES COLLECTED O/26/5O

$\begin{array}{ll}1 & N F \\ 2 & S F \\ 3 & F 1 \\ 4 & F 2 \\ 5 & P 3 \\ 6 & P 4 \\ 7 & P 5\end{array}$

13.90

60.50

.2313

14.04

$76.5 \mathrm{~J}$

46.28

$177: 00$

37.46

135,80

, 1911

95.03

324.70

.2614

.2793

15.16

6.7 .10

.2067

12.36

$76 . \overline{30}$

.2254

.1021

SAMPLES COLLECTED 10/13/66

\begin{tabular}{|c|c|c|c|c|}
\hline 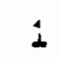 & $i ! F$ & 28.10 & 120.40 & $.22: 23$ \\
\hline 2 & $C F$ & 24.98 & 133.30 & .1374 \\
\hline 3 & $S F$ & $27.7 \%$ & 119.90 & .2317 \\
\hline 4 & $F 1$ & 27.60 & 121,90 & .2271 \\
\hline 5 & $F 2$ & 22.47 & 90.10 & .2303 \\
\hline 6 & PS & 48.98 & 273.00 & .1774 \\
\hline 7 & +1 & 21.93 & 111.00 & .3 .931 \\
\hline$e$ & $F_{2}$ & 24.50 & 144.00 & .2708 \\
\hline 9 & 53 & 22.09 & 111.60 & .2053 \\
\hline 10 & F 4 & 20,23 & 107.40 & .1884 \\
\hline 11 & P5 & 25.05 & 116.80 & .2144 \\
\hline 12 & F6 & 24.70 & 102.40 & .2412 \\
\hline 13 & 3320 & 29.33 & 137.80 & a \\
\hline 14 & 3321 & 34.41 & 146.40 & 20300 \\
\hline 15 & 3322 & 26.87 & 124,40 & .2150 \\
\hline 10 & 3324 & 25.00 & 115.00 & .2174 \\
\hline 17 & 3326 & 21.91 & 93.60 & .2341 \\
\hline 10 & 3329 & 24.31 & 96.10 & .2230 \\
\hline 19 & 3334 & 29.62. & 111.70 & .2052 \\
\hline 20 & 3335 & 29.91 & 111.20 &, 2090 \\
\hline CI & 3330 & 32.78 & 107.40 & .2790 \\
\hline 22 & 3338 & 29.18 & 90.90 & .3210 \\
\hline 23 & 3340 & 33.06 & 107.00 & .3067 \\
\hline 24 & 3342 & 19.15 & 116.00 & .1642 \\
\hline 25 & 3344 & 22.38 & 111.80 & .2002 \\
\hline 20 & 3345 & 21.99 & 80.10 & .2268 \\
\hline 27 & 3346 & 18.66 & 87.40 & .2135 \\
\hline$\angle C$ & 3347 & 28.16 & 105.30 & .2074 \\
\hline$<9$ & 3345 & 21.34 & 101.90 & .21194 \\
\hline نu & 3349 & 30.32 & 100.10 & .2845 \\
\hline 31 & 3350 & 23.32 & 107.50 & .2035 \\
\hline $3 i c$ & 3351 & 39.28 & 109.30 & .3594 \\
\hline 33 & $33 E ?$ & 23.63 & 106.30 & .2223 \\
\hline 34 & 3353 & 28.19 & 107.00 & \\
\hline
\end{tabular}


TABLE A9. (Continued 14)

CESIUH-137 IN ALFALFA (CONT,)

\begin{tabular}{|c|c|c|c|c|}
\hline $\begin{array}{l}\text { SAMFLE } \\
\text { NUMBER }\end{array}$ & $\begin{array}{l}\text { FIELU } \\
\text { LOCATION }\end{array}$ & $P=1 / 12$ & $P C I / K G$ & $k 3 / M_{2}$ \\
\hline 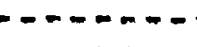 & $\ldots \ldots . .$. & ----- & ----- & $---\infty$ \\
\hline 05 & 3356 & 23.35 & 103,30 & $.245 i$ \\
\hline 30 & 3355 & 24.51 & 118.50 & .2075 \\
\hline 37 & 3357 & 25.73 & 119.30 & .2156 \\
\hline 38 & $3 j 60$ & 2.1 .69 & 137.70 & .1275 \\
\hline $3 \%$ & 3361 & 28.57 & 120,60 & .2257 \\
\hline 413 & 3.36 .5 & 26.76 & \pm 43.90 & .1000 \\
\hline $4 I$ & $336:$ & 21.29 & 105.90 & $: 1955$ \\
\hline 42 & 356 & $24.5 \%$ & 110.10 &.$<150$ \\
\hline-3 & 3367 & 25.45 & 102,20 &, 2238 \\
\hline 44 & 3309 & 69.30 & 309.30 & .1377 \\
\hline 45 & 3570 & 10.81 & 90.30 & .2193 \\
\hline $4 \dot{n}$ & 3371 & 33.92 & 51.80 & .2574 \\
\hline 47 & 3372 & 26.01 & 125.20 & .2077 \\
\hline 48 & 3374 & $\therefore 4.23$ & 96,30 & .1997 \\
\hline$A S$ & 3.376 & 19.29 & $=3.20$ & .1074 \\
\hline$\partial$ & 3377 & 25.94 & 1.2 .50 & .2306 \\
\hline 51 & 3378 & 22.40 & 113.50 & .1973 \\
\hline 52 & 3379 & 16.07 & 102.00 & .1267 \\
\hline 23 & 3380 & 25.81 & 109.10 & .2366 \\
\hline 54 & $338 \%$ & 25.05 & 110.80 & .2144 \\
\hline 20 & 3284 & 23.68 & 107.90 & .2192 \\
\hline 50 & $33 \varepsilon 6$ & 26.90 & 116.50 & .2270 \\
\hline b7 & 3390 & 26.57 & 110.10 & .2595 \\
\hline 58 & 3391 & 30,35 & $=0+.50$ & .2904 \\
\hline 59 & 5393 & 30.45 & 114.90 & .2024 \\
\hline ơ & 5396 & 25.14 & 112,90 &.$\angle \$ 70$ \\
\hline 61 & 3401 & 25.06 & $10 y .00$ & .2287 \\
\hline 62 & 3402 & 24,15 & 110.10 & .2193 \\
\hline 63 & 3404 & 29.09 & $11: 30$ & .2591 \\
\hline 64 & 3408 & 19.47 & 97.40 & .2598 \\
\hline 65 & 3405 & $=0.17$ & 11.0 .00 & .1033 \\
\hline 06 & 3411 & 27.18 & 107.90 & .2219 \\
\hline 67 & 3413 & 25.62 & 123.10 & $.2 J 31$ \\
\hline 08 & $3<15$ & 29.74 & 112.60 & .2573 \\
\hline 64 & 3410 & 25.13 & 122.00 & .2054 \\
\hline 70 & $\vec{s} 4 \dot{y}$ & 25.88 & $13 i .4 i$ & .1970 \\
\hline
\end{tabular}


TABLE A10. STRONTIUM-90 IN INDIVIDUAL ALFALFA SAMNLES

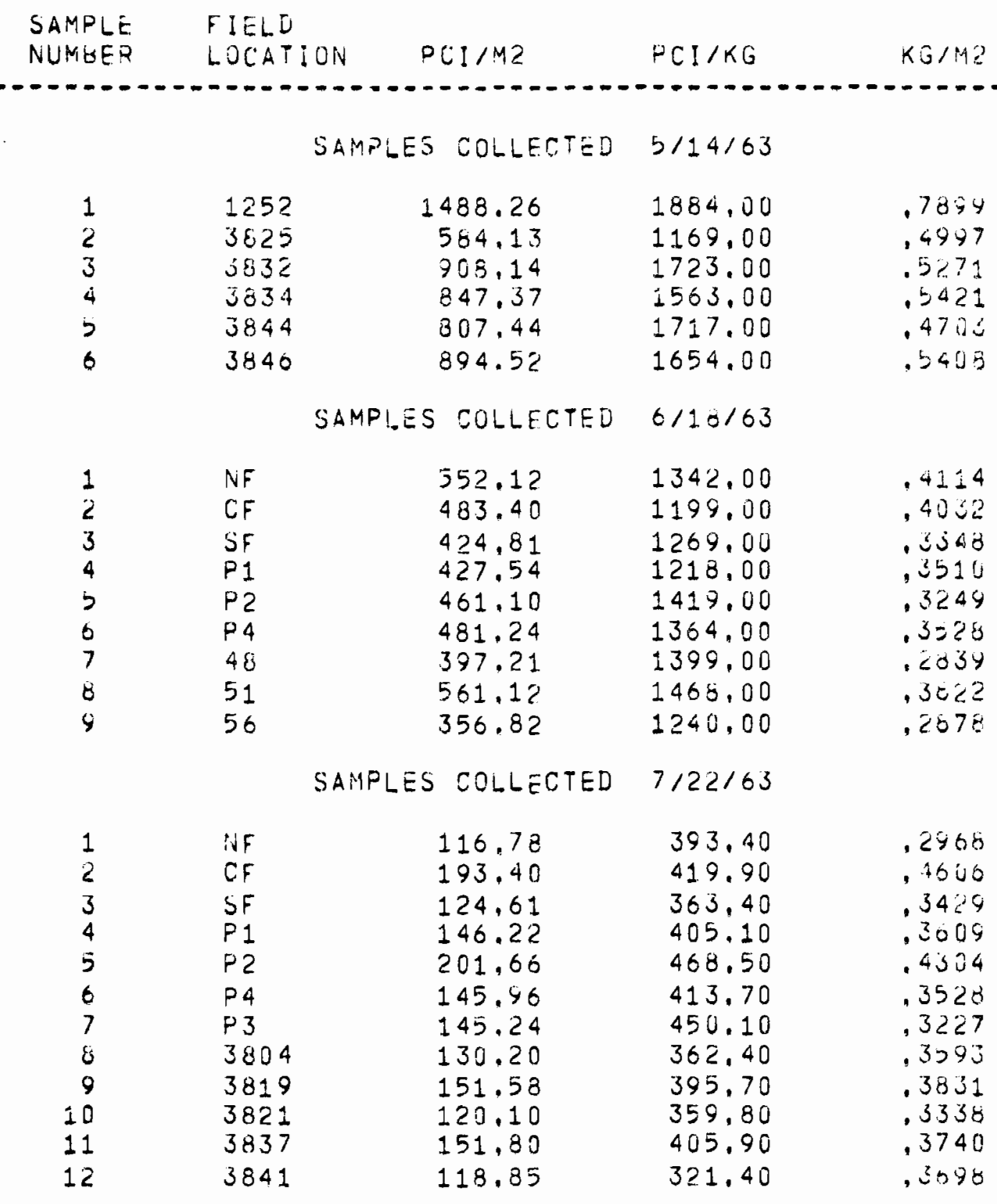

SAMPLES COLLECTED $8 / 26 / 63$

$\begin{array}{lllll}1 & N F & 142.42 & 519.10 & .2744 \\ 2 & C F & 176.22 & 593.40 & .2970 \\ 3 & P 1 & 183.96 & 515.50 & .3763 \\ 4 & F 2 & 128.40 & 459.20 & .2796 \\ 5 & P 3 & 104.36 & 486.10 & .2147 \\ 6 & P 4 & 213.48 & 589.50 & .3621 \\ 7 & 3811 & 116.05 & 475.20 & .2442 \\ 8 & 3824 & 83.58 & 318.10 & .2028\end{array}$


TABLE A10. (Continued 2)

$$
\text { STRONTIUIA-90 IN ALFALFA (CONT,) }
$$

\begin{tabular}{ccccr} 
SAMPLE & FIELD & & & \\
NUMEER & LOCATION & PCI/M2 & $P C I / K G$ & KEIM2 \\
\hline 9 & 3827 & 122.39 & 374.30 & .3270 \\
10 & 3840 & 159.47 & 528.90 & .315 \\
11 & 3843 & 89.56 & 410.10 & .2184
\end{tabular}

SAMPLES COLLECTED $10 / 6 / 63$

$\begin{array}{rlrll}1 & N F & 55.16 & 672.30 & .0820 \\ 2 & S F & 57.79 & 725.50 & .0797 \\ 3 & P 1 & 95.30 & 817.90 & .1043 \\ 4 & P 2 & 49.58 & 695.60 & .0713 \\ 5 & P 3 & 72.80 & 618.00 & .1178 \\ 6 & 169 & 141.54 & 738.30 & .1917 \\ 7 & 170 & 92.54 & 534.90 & .1300 \\ 8 & 172 & 93.08 & 702.70 & .1396 \\ 9 & 175 & 53.99 & 677.50 & .0871 \\ 11 & 177 & 92.97 & 574.10 & .1619 \\ 11 & 3792 & 80.06 & 602.50 & .1329 \\ 12 & 3813 & 48.16 & 606.40 & .0794 \\ 13 & 3313 & 125.54 & 654.80 & .1917 \\ 14 & 3828 & 76.02 & 630.60 & .1206 \\ 15 & 3829 & 70.20 & 631.80 & .1111 \\ 16 & 3839 & 83.21 & 596.20 & .1376\end{array}$

SAMPLES COLLECTED 5/14/64

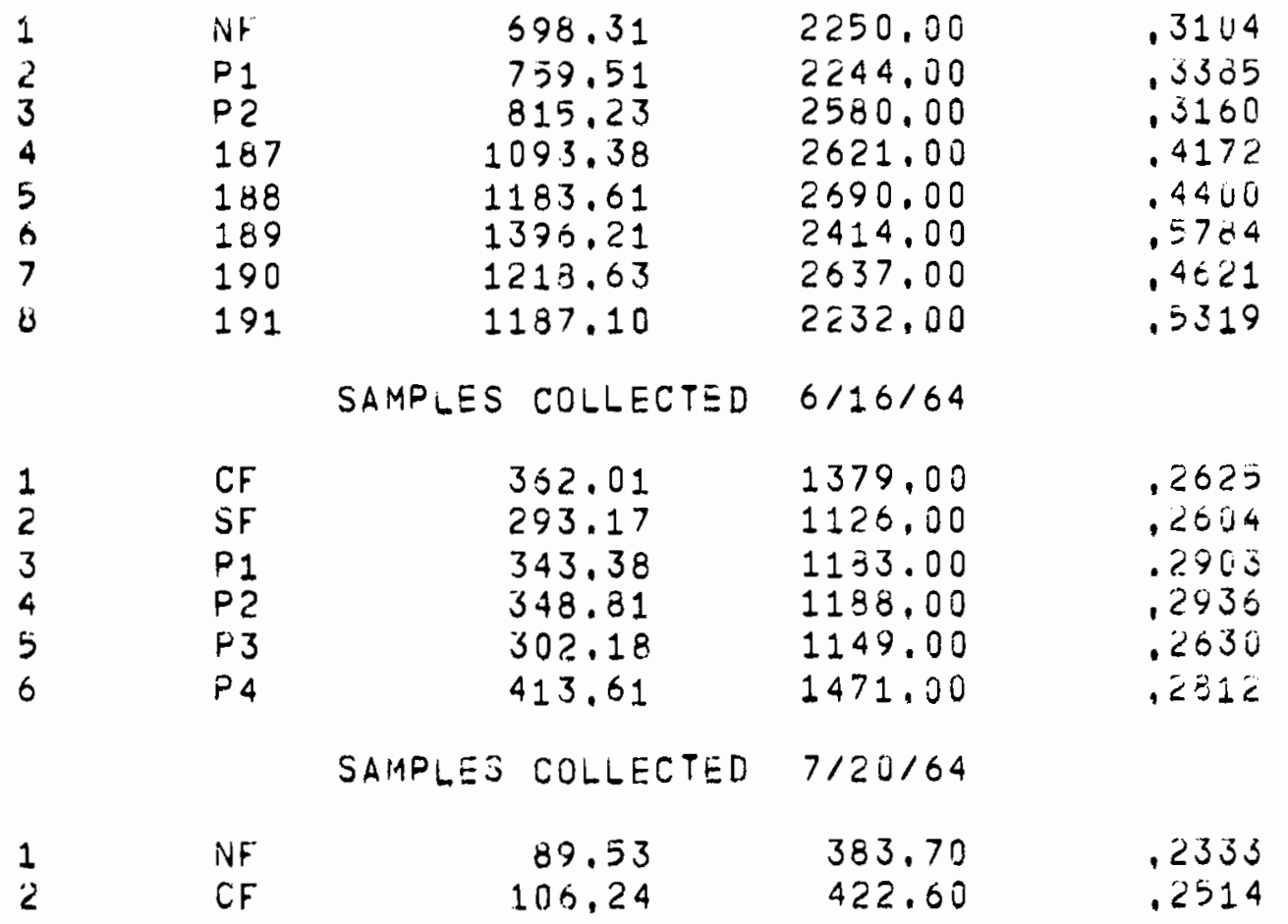


TABLE A10. (Continued 3)

$$
\text { STRONTIUM-90 IN ALFALFA (CONT, ) }
$$

\begin{tabular}{ccccc} 
SAMPLE & FIELD & & & \\
NUMEER & LUCATION & PCI/M2 & PCI/KG & $K G / M 2$ \\
\hline 3 & SF & 103.64 & 353.70 & .2930 \\
4 & $P 1$ & 102.79 & 416.00 & .2471 \\
5 & $P 3$ & 76.43 & 415.50 & .1839
\end{tabular}

SAMPLES COLLECTED $8 / 25 / 64$

$\begin{array}{lrrrl}1 & N F & 106.01 & 445.20 & .2381 \\ 2 & P 1 & 95.12 & 367.00 & .2592 \\ 3 & P 2 & 106.76 & 364.20 & .2931 \\ 4 & P 3 & 90.93 & 379.40 & .2397 \\ 5 & P 4 & 70.22 & 358.20 & .1960\end{array}$

SAMPLES COLLECTED $10 / 18 / 64$

\begin{tabular}{|c|c|c|c|}
\hline NF & 54.14 & 363.30 & .1490 \\
\hline UF & 93.97 & 702.80 & .1337 \\
\hline$P 2$ & 03.71 & 510.70 & .1247 \\
\hline$P_{4}$ & 57.38 & 474.60 & .1420 \\
\hline 581 & 73.97 & 465,00 & .1591 \\
\hline 582 & 65,22 & 465.70 & .1400 \\
\hline 583 & 57.88 & 469.40 & .1233 \\
\hline 584 & 64.35 & 385.40 & .1670 \\
\hline 585 & 69.28 & 437.50 & .1283 \\
\hline 587 & 68.48 & 385,30 & .1777 \\
\hline 588 & 54.22 & 428.90 & .1264 \\
\hline 589 & 40.48 & 328.60 & .1232 \\
\hline 59.1 & 39.23 & 356.50 & .1100 \\
\hline 593 & 86.72 & 412.20 & .2104 \\
\hline 594 & 82.61 & 384,80 & .2147 \\
\hline 595 & 55.92 & 421.60 & .1326 \\
\hline 596 & 87.84 & 423.80 & .2073 \\
\hline 597 & 35.00 & 401,10 & .2119 \\
\hline 598 & 78.98 & 423.60 & .1865 \\
\hline 599 & 70.41 & 437.70 & .1609 \\
\hline 600 & 60.44 & 417,30 & .1448 \\
\hline 602 & 74.21 & 592.10 & .1253 \\
\hline 603 & 53.29 & 477.10 & .1117 \\
\hline 604 & 61.80 & 473.20 & .1306 \\
\hline 605 & 42.94 & 386.10 & .1112 \\
\hline 606 & 47.02 & 424.10 & .1109 \\
\hline 607 & 72.35 & 450,80 & .1605 \\
\hline 608 & 52.88 & 437.30 & .1209 \\
\hline 609 & 62.55 & 393.50 & 1589 \\
\hline 610 & 51.14 & 395.20 & .1294 \\
\hline 611 & 55.52 & 414,10 & .1341 \\
\hline 612 & 80.55 & 414.50 & .1943 \\
\hline 614 & 52.79 & 377,30 & .1329 \\
\hline
\end{tabular}


TABLE A10. (Continued 4)

STRONTIUM-90 IN ALFALFA (CONT, )

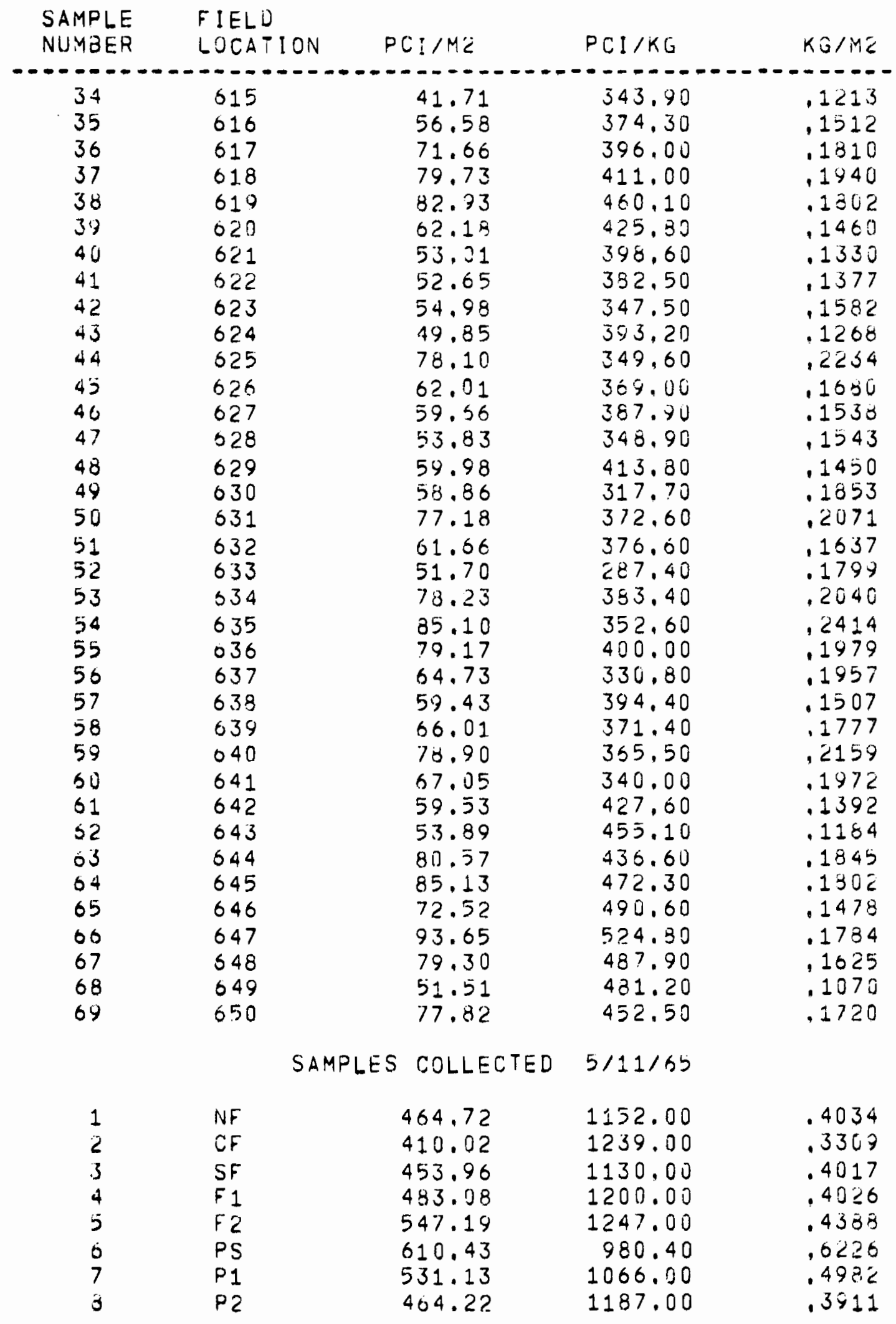


TABLE A10. (Continued 5)

STRONTIUM-90 IN ALFALFA (CONT, )

\begin{tabular}{|c|c|c|c|c|}
\hline $\begin{array}{l}\text { SAMPLE } \\
\text { NUMBER }\end{array}$ & $\begin{array}{l}\text { FIELD } \\
\text { LOCATION }\end{array}$ & $P C 1 / 112$ & $\mathrm{PCI} / \mathrm{KG}$ & $K G / M Z$ \\
\hline--- & --- & $\cdots$ & - & ------ \\
\hline 9 & $P 4$ & 578.37 & 1307.00 & .4425 \\
\hline 10 & P5 & 362.98 & 1048.00 & .3454 \\
\hline 11 & P6 & 423.97 & 1145.00 & .3703 \\
\hline 12 & 811 & 695.48 & 1310.00 & $.337 y$ \\
\hline 13 & 820 & 947.71 & 1007.00 & .5097 \\
\hline 14 & 821 & 200.12 & 958.90 & .2087 \\
\hline 15 & 823 & 515.24 & 1483.30 & .3474 \\
\hline 16 & 824 & 441.99 & 1125.00 & $.3 y 29$ \\
\hline 17 & 825 & 543.03 & 1291.00 & .4206 \\
\hline 18 & 826 & 486.67 & 1200.00 & .4050 \\
\hline 19 & 827 & 475.20 & 1104.00 & .4304 \\
\hline 20 & 828 & 495.24 & 1264.00 & .3913 \\
\hline 21 & 830 & 637.34 & 1430.20 & .4430 \\
\hline 22 & 832 & 468.48 & 1162.00 & .4032 \\
\hline $\begin{array}{l}23 \\
24\end{array}$ & $\begin{array}{l}834 \\
835\end{array}$ & $\begin{array}{l}610.70 \\
587.34\end{array}$ & $\begin{array}{l}1348.00 \\
1351.00\end{array}$ & $\begin{array}{r}.4530 \\
.4347\end{array}$ \\
\hline 25 & 836 & 503.30 & 1283.00 & .3423 \\
\hline 26 & 837 & 488.79 & 1394.00 & .3508 \\
\hline 27 & 838 & 360.12 & 1129.00 & .3190 \\
\hline 28 & 839 & 584.77 & 1268.00 & .4612 \\
\hline 29 & 840 & 624.45 & 1374.00 & .4545 \\
\hline 30 & 841 & 544.35 & 1189.00 & .4573 \\
\hline 31 & $84 \overline{3}$ & 380.15 & 1093.00 & .3533 \\
\hline 32 & 844 & 394.10 & 1451.00 & .2716 \\
\hline 33 & 845 & 459.54 & 1183.00 & .3885 \\
\hline 34 & 848 & 411.58 & 1341.00 & .3062 \\
\hline 35 & 849 & 427.24 & 1309.00 & .3204 \\
\hline 36 & 850 & 373.73 & 1166.00 & .3205 \\
\hline 37 & 851 & 492.63 & 1141.00 & .4318 \\
\hline 38 & 852 & 557.49 & 1227.00 & .4544 \\
\hline 39 & 853 & 608.42 & 1510.00 & $4 \cup 29$ \\
\hline 40 & 854 & 448.86 & 1237.00 & .3629 \\
\hline 41 & 855 & 243.52 & 934.60 & .2473 \\
\hline 42 & 856 & 547.50 & 1348.00 & .4052 \\
\hline 43 & 858 & 574.98 & 1223.00 & .4632 \\
\hline 44 & 860 & 3013.62 & 1055.00 & $.28 y 0$ \\
\hline 45 & 861 & 586.51 & 1329.00 & .4413 \\
\hline 46 & $86 \overline{2}$ & 410.45 & 1453.00 & .2500 \\
\hline 47 & 863 & 540.51 & 1394.00 & .3877 \\
\hline 48 & 864 & 474.88 & 1271.00 & .3736 \\
\hline 49 & 865 & 363.88 & 1204.00 & .3022 \\
\hline 50 & 866 & 452.18 & 1079.00 & $.41 \ni 1$ \\
\hline 51 & 867 & 489.19 & 1358.00 & .3602 \\
\hline 52 & 869 & 483.29 & 1235.00 & .3913 \\
\hline 53 & 870 & 577.08 & 1232.00 & .4673 \\
\hline 54 & 871 & 500.06 & 1247.00 & .4015 \\
\hline 55 & 873 & 443.51 & 1315.00 & .3373 \\
\hline
\end{tabular}


TABLE A10. (Continued 6)

STRONTIUM-90 IN ALFALFA (CONT,)

\begin{tabular}{|c|c|c|c|c|}
\hline $\begin{array}{l}\text { SAMPLE } \\
\text { NUMBER }\end{array}$ & $\begin{array}{l}\text { FIELD } \\
\text { LOCATION }\end{array}$ & $P C I / M 2$ & $P C I / K G$ & $K r_{2} / M_{2}$ \\
\hline$\ldots$ & - - - - - & ----- & $-\cdots$ & $---\infty$ \\
\hline 50 & 875 & $437.8 ?$ & 1323.00 & .33199 \\
\hline 57 & 876 & 627.86 & 1294.00 & .4852 \\
\hline 53 & 877 & 543.36 & 1131.00 & .4804 \\
\hline 59 & 878 & 632.06 & 1397.00 & .4524 \\
\hline 60 & 879 & 364.57 & 1213.00 & .3000 \\
\hline 61 & 880 & 544.40 & 1243.00 & .4380 \\
\hline 62 & 882 & 572.33 & 1269.01 & .4510 \\
\hline 65 & 383 & 531.35 & 1246.30 & .4258 \\
\hline 54 & 884 & 633.29 & 1575.00 & .4021 \\
\hline 65 & 885 & 851.75 & 1577.00 & .5401 \\
\hline 56 & 886 & 387.13 & 1259.00 & .3075 \\
\hline 57 & 887 & 502.56 & 1444.00 & .3480 \\
\hline 68 & 889 & 427.31 & 1170.00 & .3069 \\
\hline & SAM & S COLLECTED & $6 / 16 / 65$ & \\
\hline 1 & $N F$ & 251.12 & 986.70 & .2345 \\
\hline 2 & CF & 205.50 & 790.00 & .2601 \\
\hline 3 & SF & 284.29 & 1000.00 & .2843 \\
\hline 4 & $F_{1}$ & 271.12 & 932.90 & .2900 \\
\hline 5 & $F_{2}$ & 334.92 & 1165.00 & .2874 \\
\hline 6 & $F 2$ & 248.97 & 866,30 & .2874 \\
\hline 7 & $P 1$ & 286.89 & 964.90 & .2973 \\
\hline $\bar{z}$ & $P 2$ & 321.44 & 917.30 & .3504 \\
\hline 9 & $P 3$ & 243.65 & 865.80 & .2014 \\
\hline 10 & $P 4$ & 283,28 & 992.70 & .2854 \\
\hline 11 & PG & 376.15 & 1010.00 & .3724 \\
\hline 12 & 1102 & 221.54 & 865,60 & .2559 \\
\hline 13 & 1103 & 281.26 & 797.20 & .3528 \\
\hline 14 & 1104 & 325.51 & 750.60 & .4337 \\
\hline 15 & 1105 & 248.61 & 750.70 & .3312 \\
\hline 10 & 1106 & 236.16 & 762.10 &, $3 \cup y 5$ \\
\hline 17 & 1108 & 230.09 & 883.70 & .2504 \\
\hline 18 & 1110 & 209.04 & $718 \cdot 10$ & .2911 \\
\hline 19 & 1111 & 295.07 & 823.20 & .3594 \\
\hline 20 & 1112 & 231.83 & 784.10 & .5722 \\
\hline 21 & 1113 & 282.11 & 720.90 & .3913 \\
\hline 22 & 1113 & 339.71 & 868.10 & .3913 \\
\hline 23 & 1114 & 236.47 & 789.00 & .2947 \\
\hline 24 & 1115 & 298.46 & 904.50 & .3300 \\
\hline 25 & 1117 & 266.46 & 830.10 & .3210 \\
\hline 26 & 1120 & 298.40 & 817.50 & .3650 \\
\hline 27 & 1122 & 221.47 & 831.90 & .2002 \\
\hline 28 & 1123 & 291.59 & 885.50 & .3294 \\
\hline 29 & 1124 & 313.23 & 886.30 & .3534 \\
\hline 30 & 1126 & 260.78 & 875.70 & .2975 \\
\hline 31 & 1127 & 263.76 & 846.60 & .3110 \\
\hline
\end{tabular}


TABLE A10. (Continued 7)

STRUNTIUM-90 I IN ALFALFA (CONT.)

\begin{tabular}{|c|c|c|c|c|}
\hline $\begin{array}{l}\text { SAMPLE } \\
\text { NUMBER }\end{array}$ & $\begin{array}{l}\text { FIELD } \\
\text { LOCATION }\end{array}$ & $P C I / M 2$ & $P C I / K G$ & $K G / Y C$ \\
\hline$-----n$ & $\ldots-\ldots$ & -..- & 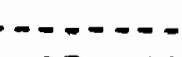 & ----- \\
\hline 32 & 1128 & 197.18 & 871.40 & .2263 \\
\hline 33 & 1130 & 207.85 & 913.70 & .2275 \\
\hline 34 & 1131 & 269.35 & 882.50 & .3052 \\
\hline 35 & 1133 & 256.25 & 824.40 & .3106 \\
\hline 36 & 1134 & 234.31 & 776.20 & .3017 \\
\hline 37 & 1136 & 259.27 & 848.80 & .5055 \\
\hline 38 & 1136 & 264.31 & 866.00 & .3049 \\
\hline 39 & 1139 & 296.15 & 801.90 & .3430 \\
\hline 40 & 1141 & 291.11 & 785.70 & .3735 \\
\hline 41 & 1143 & 254.37 & 360.80 & .2928 \\
\hline 42 & 1145 & 269.61 & 746.20 & .3015 \\
\hline 43 & 1147 & 249.74 & 725.30 & .3443 \\
\hline 44 & 1148 & 235.11 & 727.00 & .3234 \\
\hline 45 & 11.49 & 207.07 & 751.70 & .2753 \\
\hline $\begin{array}{l}40 \\
47\end{array}$ & $\begin{array}{l}1150 \\
1151\end{array}$ & $\begin{array}{l}226.56 \\
272.51\end{array}$ & $\begin{array}{l}759.30 \\
710.70\end{array}$ & $\begin{array}{l}.2733 \\
.3834\end{array}$ \\
\hline 48 & 1152 & 301.86 & 714.40 & .4225 \\
\hline 49 & 1153 & 245.03 & 746.10 & .3294 \\
\hline לu & 1155 & 257.27 & 048.90 & .3462 \\
\hline 51 & 1156 & 249.40 & .752 .00 & .3316 \\
\hline 52 & 1157 & 286.44 & 730.40 & .3922 \\
\hline 53 & $\$ 161$ & 301.21 & 750.00 & .4010 \\
\hline 54 & 1161 & 304.98 & 759.40 & .4316 \\
\hline 35 & 1163 & 289.97 & 844.20 & .3435 \\
\hline 56 & 1164 & 272.52 & 741.50 & .357 \\
\hline 57 & 1168 & 251.95 & 833.00 & .3075 \\
\hline 58 & 1169 & 298.18 & 816,90 & .3650 \\
\hline
\end{tabular}

SAMPLES COLLECTED 7/20/OO5

$\begin{array}{rlrll}1 & N F & 37.51 & 310.70 & .2317 \\ 2 & S F & 03.12 & 299.70 & .3107 \\ 3 & P S & 140.07 & 360.30 & .3802 \\ 4 & P 1 & 103.17 & 303.20 & .3403 \\ 5 & P 2 & 121.17 & 343.80 & .3525 \\ 6 & P 3 & 101.23 & 329.10 & .3076 \\ 7 & P 3 & 110.98 & 360.80 & .3076 \\ 8 & P 4 & 109.54 & 357.20 & .3057 \\ 9 & P 5 & 118.59 & 357.20 & .3320 \\ 10 & P 6 & 106.76 & 294.90 & .3020 \\ 11 & 1380 & 121.64 & 407.00 & .2959 \\ 12 & 1382 & 82.39 & 374.40 & .2201 \\ 13 & 1383 & 105.38 & 389.00 & .2704 \\ 14 & 1384 & 93.08 & 312.30 & .2980 \\ 15 & 1385 & 92.89 & 381.30 & .2430 \\ 16 & 1380 & 75.60 & 360.40 & .2098 \\ 17 & 1387 & 107.89 & 419.20 & .2374\end{array}$


TABLE A10. (Continued 8)

STHONTIUM-9O IN AL.FALFA (CONT.)

\begin{tabular}{|c|c|c|c|c|}
\hline $\begin{array}{l}\text { SAMPLE } \\
\text { NUMBER }\end{array}$ & $\begin{array}{l}\text { FIELJ } \\
\text { LOCATION }\end{array}$ & $P C I / M Z$ & $P C I / K B$ & $\mathrm{Ku} / \mathrm{ML}$ \\
\hline..- &..-- & $-\ldots$ & $=--$ & ----- \\
\hline 18 & 1389 & 69.12 & 483.60 & $: 1429$ \\
\hline 19 & 1390 & 85.91 & 393.80 & .2181 \\
\hline 20 & 1390 & 95.40 & 4.37 .30 & .2151 \\
\hline 21 & 1391 & 124.74 & 386.00 & .3232 \\
\hline 22 & 1392 & 141.75 & 371.20 & .3819 \\
\hline 23 & 1393 & 120.41 & 385.30 & bاל \\
\hline 24 & 1394 & 105.09 & 395.10 & .2050 \\
\hline 25 & 1395 & 148.23 & 462.80 & .3203 \\
\hline 20 & 1396 & 96.52 & 360.30 & .2079 \\
\hline 27 & 1396 & 100.01 & 373.30 & .2679 \\
\hline 28 & 1397 & 84.40 & 379.80 & .2222 \\
\hline 20 & 1398 & 116.16 & 358.80 & .3233 \\
\hline jo & 1400 & 101.31 & 413.40 & .2451 \\
\hline 31 & 1403 & 138.45 & 435.50 & .3172 \\
\hline 32 & 1404 & 111.39 & 356.70 & .3123 \\
\hline 33 & 1405 & 71.59 & 418.60 & .1710 \\
\hline 34 & 1406 & 95.26 & 345.10 & .2760 \\
\hline 55 & 1407 & 131.32 & 477.60 & .2750 \\
\hline 36 & 1408 & 84.75 & 420.30 & .2016 \\
\hline 37 & 1409 & 114.84 & 331.00 & .3470 \\
\hline 38 & 1411 & 110.91 & 370.80 & $.29 \ni 1$ \\
\hline 39 & 1412 & 94.78 & 388.10 & .2442 \\
\hline 40 & 1413 & 97.79 & 420.80 & .2364 \\
\hline 41 & $14: 4$ & 107.26 & 392.50 & .2733 \\
\hline 42 & 1415 & 108.36 & 339.60 & .3181 \\
\hline 43 & 1416 & $126.8 ?$ & 452.20 & .2305 \\
\hline 44 & 1417 & 113.75 & 363.30 & .3131 \\
\hline 45 & 1418 & 137.10 & 405.50 & .3381 \\
\hline 46 & 1419 & 125.22 & 332.50 & .3706 \\
\hline $4 \%$ & 1421 & 125.52 & 360.90 & .3478 \\
\hline 48 & 1422 & 93.08 & 310.20 & .3001 \\
\hline 49 & 1423 & 133.49 & 429.80 & .3106 \\
\hline 50 & 1424 & 104.83 & 398.60 & .2630 \\
\hline 51 & 1425 & 98.83 & 350.90 & .2817 \\
\hline 52 & 1426 & 116.41 & 388.70 & .2995 \\
\hline 53 & 1423 & 93.38 & 338.00 & .2763 \\
\hline 54 & 1429 & 120.11 & 357.40 & .3551 \\
\hline 55 & 1431 & 114.78 & 377.10 & .3044 \\
\hline 56 & 1433 & 125.73 & 331.10 & $.37 ; 7$ \\
\hline 57 & 1434 & 160.98 & 433.50 & .3714 \\
\hline 58 & 1436 & 105.71 & 333.90 & .3125 \\
\hline 57 & 1438 & 134.16 & 330.90 & .4054 \\
\hline 60 & 1439 & 101.37 & 370.60 & .2735 \\
\hline 61 & 1441 & 143.88 & 372.80 & .3859 \\
\hline 62 & 1442 & 100.02 & 369.40 & .2708 \\
\hline 63 & 1443 & 128.20 & 374.00 & .3428 \\
\hline 64 & 1444 & 114.36 & 402.60 & .2840 \\
\hline
\end{tabular}


TABLE A10. (Continued 9)

STRONTIUM-9O IN ALFALFA (CONT.)

\begin{tabular}{ccccc} 
SAMPLE & FIELU & & & \\
NUMOER & LOCATION & PCI/M2 & PCI/KG & KG/M2 \\
\hline 60 & 1445 & 146.61 & 412.20 & .3557 \\
60 & 1446 & 131.43 & 406.70 & .3232 \\
57 & 1447 & 112.50 & 455.30 & .2471 \\
68 & 1440 & 170.60 & 479.80 &, 3555 \\
60 & 1449 & 71.84 & 370.60 & .1908
\end{tabular}

SAMPLES COLLECTED $8 / 26 / 55$

\begin{tabular}{|c|c|c|c|c|}
\hline$\frac{1}{3}$ & $\begin{array}{l}C F \\
S F \\
P S\end{array}$ & $\begin{array}{l}76.45 \\
82.39 \\
95.98\end{array}$ & $\begin{array}{l}312.70 \\
304.40 \\
341.80\end{array}$ & $\begin{array}{l}.3084 \\
.2707 \\
.2008\end{array}$ \\
\hline $\begin{array}{l}4 \\
5 \\
6\end{array}$ & $\begin{array}{l}F 1 \\
P 2 \\
P 3\end{array}$ & $\begin{array}{r}100.95 \\
114.27 \\
81.48\end{array}$ & $\begin{array}{l}332.70 \\
382.50 \\
365.70\end{array}$ & $\begin{array}{l}.3034 \\
.2988 \\
.2227\end{array}$ \\
\hline $\begin{array}{l}7 \\
3 \\
0\end{array}$ & $\begin{array}{l}P 6 \\
1661 \\
166=\end{array}$ & $\begin{array}{r}100.61 \\
79.34\end{array}$ & $\begin{array}{l}33.10 \\
341.30 \\
475.10\end{array}$ & $\begin{array}{l}.5200 \\
.2294 \\
.2530\end{array}$ \\
\hline $\begin{array}{l}10 \\
11 \\
12\end{array}$ & $\begin{array}{l}1663 \\
1664 \\
1665\end{array}$ & $\begin{array}{r}35.64 \\
37.37 \\
100.26\end{array}$ & $\begin{array}{l}334.03 \\
351.90 \\
360.70\end{array}$ & $\begin{array}{l}.2504 \\
.2485 \\
.2779\end{array}$ \\
\hline $\begin{array}{l}13 \\
14\end{array}$ & $\begin{array}{l}1667 \\
1668\end{array}$ & $\begin{array}{l}125.13 \\
110.04\end{array}$ & $\begin{array}{r}432.50 \\
-399.70\end{array}$ & $\begin{array}{l}.2893 \\
.2733\end{array}$ \\
\hline 15 & 1669 & 107.47 & 395.50 & .2717 \\
\hline $\begin{array}{l}16 \\
17\end{array}$ & $\begin{array}{l}1670 \\
1672\end{array}$ & $\begin{array}{l}119.49 \\
11.0 .98\end{array}$ & $\begin{array}{l}397.40 \\
376.90\end{array}$ & $\begin{array}{l}.3007 \\
.2345\end{array}$ \\
\hline 18 & 1673 & 93.01 & 377.50 & .2464 \\
\hline $\begin{array}{l}19 \\
20\end{array}$ & $\begin{array}{l}1676 \\
1677\end{array}$ & $\begin{array}{r}97.28 \\
101.86\end{array}$ & $\begin{array}{l}410.4 \mathrm{~J} \\
428.00\end{array}$ & $\begin{array}{l}.2370 \\
.2330\end{array}$ \\
\hline 21 & 1678 & 109.05 & 440.90 & $.247^{\circ}$ \\
\hline 22 & 1679 & 99.82 & 402.60 & .2475 \\
\hline 23 & 1680 & 92.35 & 397.30 & .2321 \\
\hline 24 & 1681 & 74.71 & 370.20 & d 2550. \\
\hline 25 & 1682 & 78.01 & 390.10 & .2000 \\
\hline 25 & 1683 & 108.87 & 398.90 & .2729 \\
\hline 27 & 1685 & 34.29 & 412.30 & .2030 \\
\hline 23 & 1686 & 90.92 & $412 \cdot 50$ & .2204 \\
\hline 29 & 1687 & 106.90 & 410.00 & .2047 \\
\hline 30 & 1688 & 80.37 & 337.40 & .2397 \\
\hline 31 & 1689 & 80.25 & 369.90 & .2170 \\
\hline 32 & 1691 & 88.70 & 404.50 & .2192 \\
\hline 33 & 1692 & 75.85 & 317.90 & .2386 \\
\hline 34 & 1693 & 92.16 & 352.20 & .2617 \\
\hline 35 & 1694 & 93.42 & 336.70 & .2775 \\
\hline 36 & 1696 & 93.75 & 345,00 & .2717 \\
\hline 37 & 1697 & 32.91 & 433.80 & .1911 \\
\hline 30 & 1698 & 93.80 & 423.50 & .2215 \\
\hline 39 & 1699 & 84.20 & 333.30 & .2522 \\
\hline
\end{tabular}


TABLE A10. (Continued 10)

STRONTIUM-90 IN ALFALFA (CONT, )

\begin{tabular}{|c|c|c|c|c|}
\hline $\begin{array}{l}\text { SAMPLE } \\
\text { NUMEER }\end{array}$ & $\begin{array}{l}\text { FIELD } \\
\text { LOCATION }\end{array}$ & $P C I / M 2$ & $P C I / K G$ & $K G / M 2$ \\
\hline 政 & $=--$ & --- & - &..---- \\
\hline $\begin{array}{l}4 u \\
41\end{array}$ & $\begin{array}{l}1700 \\
1701\end{array}$ & $\begin{array}{r}103.29 \\
90.03\end{array}$ & $\begin{array}{l}417.20 \\
376.20\end{array}$ & $\begin{array}{r}.2476 \\
2393\end{array}$ \\
\hline 42 & 1702 & 105.50 & 399.50 & $\begin{array}{l}.2070 \\
.2641\end{array}$ \\
\hline $4 \overline{3}$ & 1703 & 87.86 & 351.50 & .2500 \\
\hline 44 & 1704 & 94.30 & 356.30 & .2647 \\
\hline 45 & 1706 & 96.29 & 321.00 & .3000 \\
\hline 46 & 1707 & 109.25 & 415.80 & .2623 \\
\hline 47 & 1708 & 81.10 & 408.50 & .1785 \\
\hline 48 & 1709 & 101.70 & 397.90 & .2556 \\
\hline 49 & 1711 & 93.38 & 322.50 & .2895 \\
\hline 50 & 1712 & 88.38 & 389.30 & .2283 \\
\hline 51 & 1713 & 76.78 & 321.00 & $: 2392$ \\
\hline 52 & $\begin{array}{l}1714 \\
1715\end{array}$ & $\begin{array}{r}99.33 \\
101.35\end{array}$ & $\begin{array}{l}392.80 \\
390.70\end{array}$ & $\begin{array}{r}.2541 \\
2603\end{array}$ \\
\hline 53 & 1715 & 101.35 & 380.70 & $\begin{array}{l}.2002 \\
3053\end{array}$ \\
\hline $\begin{array}{l}54 \\
55\end{array}$ & $\begin{array}{l}1716 \\
1717\end{array}$ & $\begin{array}{l}111.53 \\
115.11\end{array}$ & $\begin{array}{l}365.60 \\
418.30\end{array}$ & .23752 \\
\hline 50 & 1718 & 125.54 & 413.10 & .5039 \\
\hline 57 & 1719 & 105.05 & 470.20 & .2234 \\
\hline 58 & 1722 & 114.99 & 508,70 & .2260 \\
\hline 59 & 1723 & 118.44 & 455.10 & .2602 \\
\hline 60 & 1724 & 134.80 & 522.30 & .2501 \\
\hline 6.1 & 1725 & 147.53 & 462.00 & .3193 \\
\hline \multirow{3}{*}{$\begin{array}{l}62 \\
63\end{array}$} & 1727 & 89.18 & 450.80 & .1978 \\
\hline & 1728 & 165.75 & 505.60 & .3278 \\
\hline & \multicolumn{3}{|c|}{ SAMPLES } & \\
\hline 1 & NF & 47.82 & 339.40 & .1409 \\
\hline 2 & $C F$ & 51.38 & 342.40 & .1515 \\
\hline 3 & $S F$ & 47.18 & 287.30 & .1042 \\
\hline 4 & $F_{1}$ & 52.17 & 350,40 & .1489 \\
\hline 5 & $\mathrm{~F} 2$ & 55.36 & 350.30 & .1860 \\
\hline 6 & $P S$ & 60.08 & 321.40 & .1869 \\
\hline 7 & $P_{1}$ & 65.96 & 345,90 & 1904 \\
\hline 8 & $P \overline{3}$ & 52.06 & 352,20 & .1478 \\
\hline 9 & $P_{4}$ & 23.26 & 249.00 & .0934 \\
\hline 10 & P5 & 59.91 & 352.00 & .1702 \\
\hline 11 & P6 & 59.07 & 366.70 & .1004 \\
\hline 12 & 1939 & 49.66 & 359.50 & .1381 \\
\hline 13 & 1941 & 72.66 & 402,10 & .1807 \\
\hline 14 & 1942 & 68.46 & 420.30 & .1629 \\
\hline 15 & 1943 & 31.00 & 378.80 & .2138 \\
\hline 16 & 1946 & 66.38 & 375.50 & .1768 \\
\hline 17 & 1947 & 68.50 & 391.50 & .1752 \\
\hline 18 & 1948 & 33.02 & 470,60 & .1764 \\
\hline 17 & 1949 & 66.06 & 422.60 & .1563 \\
\hline 20 & 1950 & 71.15 & 363.20 & .1959 \\
\hline
\end{tabular}


TABLE A10. (Continued 11)

STRONTIUM-9U IN ALFALFA (CONT.)

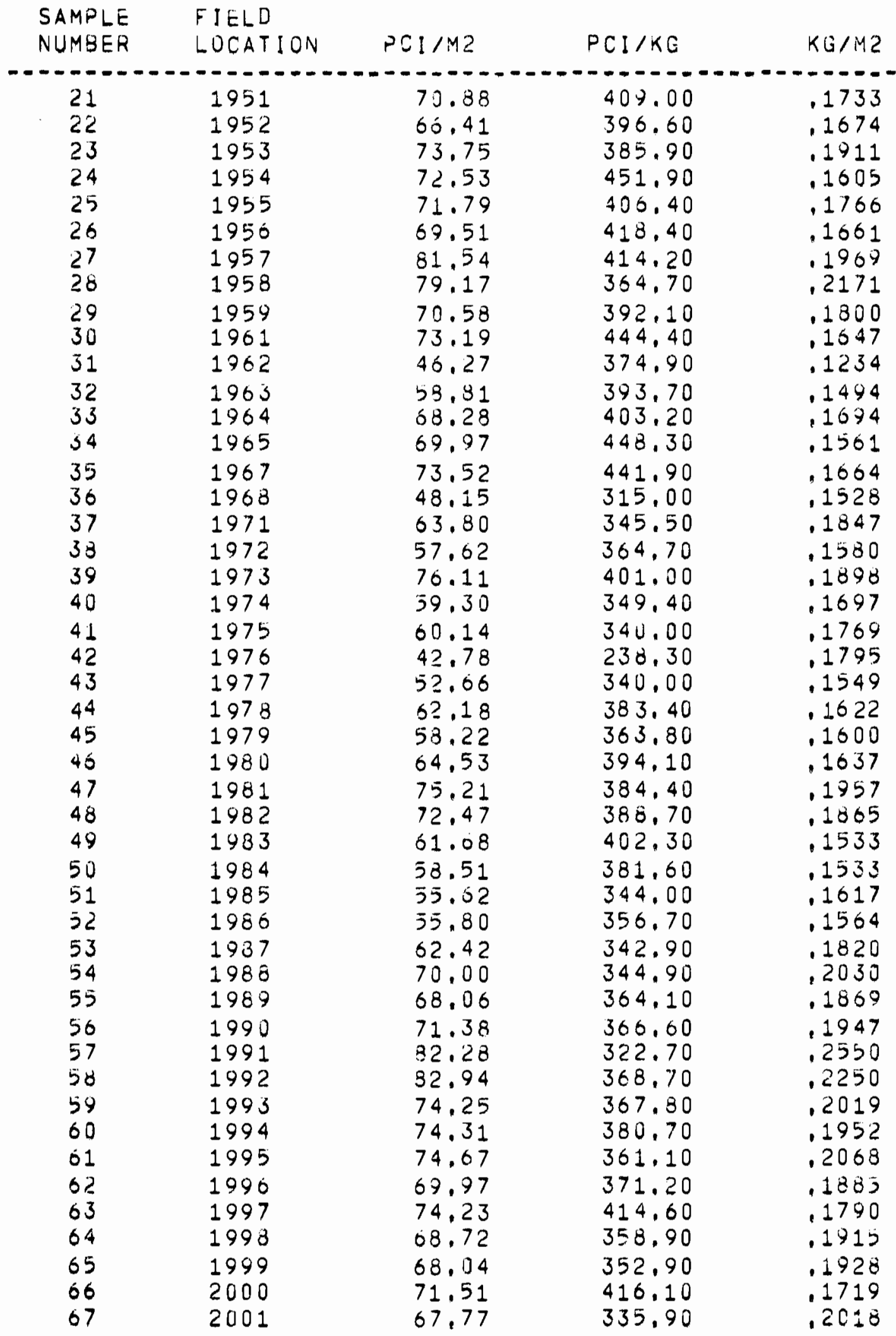


TABLE A10. (Continued 12)

STRONTIUM-9J IN ALFALFA (CONT,)

\begin{tabular}{|c|c|c|c|c|}
\hline $\begin{array}{l}\text { SAMPLE } \\
\text { NUMBER }\end{array}$ & $\begin{array}{l}\text { FIELD } \\
\text { LOCATION }\end{array}$ & $P C I / M Z$ & $P C I / K G$ & $K G / M Z$ \\
\hline$------\infty$ & $0-----$ & $--\ldots---$ & $--m--\infty$ & $-\infty$ \\
\hline 68 & 2002 & 60.23 & 420.70 & .1432 \\
\hline 69 & 2003 & 74.49 & 418.00 &. \pm 782 \\
\hline 70 & 2005 & 86.97 & 389.90 & .2231 \\
\hline 71 & 2006 & 60.10 & 443,50 & .1355 \\
\hline 72 & 2007 & 77.39 & 457.00 & .1707 \\
\hline 73 & 2008 & 74.42 & 434.50 & .2173 \\
\hline & SAl & ES COLLECTED & $5 / 11 / 66$ & \\
\hline 1 & NF & 350.61 & 777.60 & .4509 \\
\hline 2 & $C F$ & 451.10 & 858,40 & .5255 \\
\hline 3 & $S F$ & 311.35 & 047.10 & 4811 \\
\hline 4 & $F_{1}$ & 246.77 & 633.30 & .3887 \\
\hline 5 & $F 2$ & 292.54 & 750.30 & .3899 \\
\hline 6 & PS & 269.96 & 692,40 & .3399 \\
\hline 7 & P 1 & 359.53 & 801.00 & .4489 \\
\hline 8 & $P 2$ & 352.13 & 784.50 & .4489 \\
\hline 9 & $P 3$ & 308.63 & 725.70 & .4253 \\
\hline 10 & $P 4$ & 319.52 & 689.80 & .4632 \\
\hline 11 & Pó & 396.14 & 750.40 & .5279 \\
\hline 12 & 2320 & 398.48 & 756.20 & .5270 \\
\hline 13 & 2321 & 492.02 & 852.80 & .5764 \\
\hline 14 & 2322 & 500.69 & 769,00 & .6511 \\
\hline 15 & 2.323 & 368.60 & 660.80 & .5578 \\
\hline 16 & 2325 & 380.53 & 722.30 & .2268 \\
\hline 17 & 2320 & 422.58 & 710.30 & .5883 \\
\hline 18 & 2323 & 376.26 & 725.40 & .5187 \\
\hline 19 & 2329 & 334.29 & 665.90 & .5029 \\
\hline 20 & 2331 & 442.25 & 681.50 & .6439 \\
\hline 21 & 2332 & 393.96 & 668.30 & .5395 \\
\hline 22 & 2333 & 517.72 & 727.90 & .7113 \\
\hline 23 & 2334 & 471.05 & 698.70 & .0742 \\
\hline 24 & 2335 & 452.31 & 743.00 & .0088 \\
\hline 25 & 2337 & 321.26 & 740.60 & .4338 \\
\hline 26 & $? 338$ & 460.93 & 754.80 & .0107 \\
\hline 27 & 2339 & 488.61 & 753.90 & .0401 \\
\hline 28 & 2341 & 414.46 & 622.50 & .0658 \\
\hline 29 & 2342 & 578.75 & 870.50 & .6048 \\
\hline 30 & 2343 & 428.12 & 825.00 & .5189 \\
\hline 31 & 2344 & 417.39 & 789.40 & .5267 \\
\hline 32 & 2345 & 480.73 & 814.00 & .5906 \\
\hline 33 & 2346 & 410.57 & 838.10 & .4039 \\
\hline 34 & 2347 & 530.59 & 864.30 & .5139 \\
\hline 35 & 2343 & 374.12 & 900.70 & .4154 \\
\hline 36 & 2349 & 445.11 & 789.00 & .5041 \\
\hline 37 & 2350 & 404.68 & 882.30 & .4587 \\
\hline 38 & 2351 & 495.24 & 834.00 & .5938 \\
\hline
\end{tabular}

A. 54 
TABLE A10. (Continued 13)

STRONTIUM-9O IN ALFALFA (CONT, )

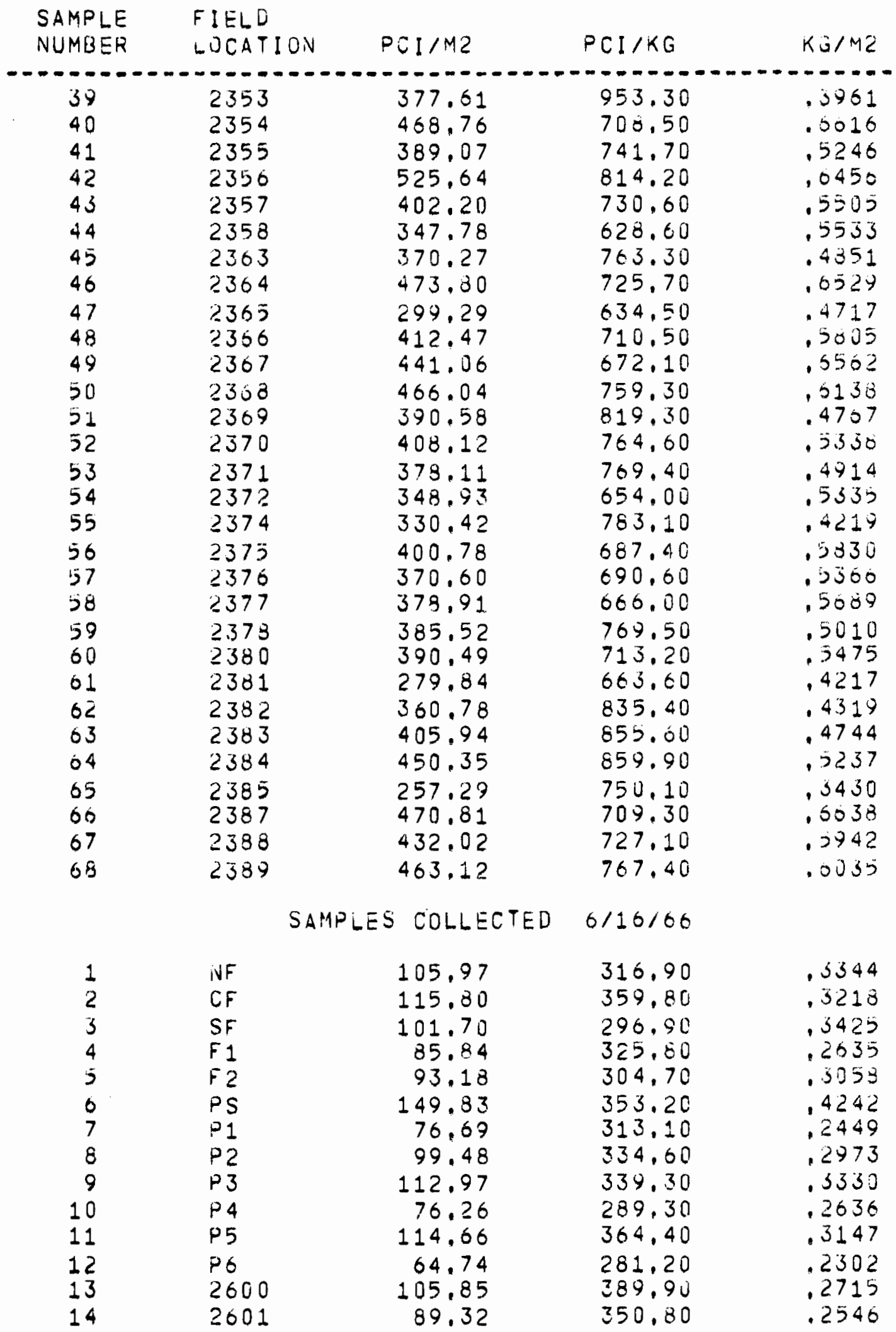


TABLE A10. (Continued 14)

STRONTIUM-90 IN ALFALFA (CONT.)

\begin{tabular}{|c|c|c|c|c|}
\hline $\begin{array}{l}\text { SAMPLE } \\
\text { NUMEER }\end{array}$ & $\begin{array}{l}\text { FIELD } \\
\text { LOCATION }\end{array}$ & $P C ! / M 2$ & $\mathrm{PCl} / \mathrm{KG}$ & $K G / M 2$ \\
\hline 15 & 2603 & 106.77 & 405,60 & .2632 \\
\hline 16 & 2604 & 72.75 & 374.10 & .1745 \\
\hline 17 & 2605 & 133,01 & 397.20 & .3332 \\
\hline 13 & 2606 & 64,16 & 365.70 & .1755 \\
\hline 19 & 2507 & 98.81 & 354.90 & .2784 \\
\hline 20 & 2603 & 111.87 & 367.40 & .3045 \\
\hline 21 & 2609 & 64.79 & 343.30 & .1887 \\
\hline 22 & 2610 & 76.88 & 376.60 & $.2 J 42$ \\
\hline 23 & 2612 & 97.28 & 376.90 & .2581 \\
\hline 24 & 2613 & 92.63 & 383.40 & .2410 \\
\hline 25 & 2614 & 117.68 & 395.00 & .2979 \\
\hline 26 & 2617 & 119.41 & 394.80 & .3225 \\
\hline 27 & 2618 & 116.04 & 373.60 & .3100 \\
\hline 28 & 2019 & 114.26 & 385.70 & .2962 \\
\hline 29 & 2620 & 109.99 & 348.90 & .3153 \\
\hline 30 & 2621 & 103.41 & 377.90 & .2736 \\
\hline 31 & 2622 & 99.60 & 371.30 & .2683 \\
\hline 32 & 2623 & 150.83 & 493.60 & .3056 \\
\hline 33 & 2624 & 102.35 & 363.70 & .2814 \\
\hline 34 & 2626 & 144.01 & 436.90 & .3296 \\
\hline 35 & 2628 & 94.34 & 326.90 & .2886 \\
\hline 36 & 2629 & 104.22 & 308.80 & .3375 \\
\hline 37 & 2630 & 115.29 & 327.10 & .3525 \\
\hline 38 & 2631 & 148,46 & 361.80 & .4103 \\
\hline 39 & 2633 & 108.95 & 325.70 & .3345 \\
\hline 40 & 2634 & 117.56 & 320.60 & .3667 \\
\hline 41 & 2637 & 94.22 & 333.40 & .2826 \\
\hline 42 & 2638 & 96.22 & 316,00 & .3045 \\
\hline 43 & 2640 & 126.37 & 294.00 & .4298 \\
\hline 44 & 2641 & 107.53 & 317,70 &., 3335 \\
\hline 45 & 2643 & 137.24 & 369.20 & .5717 \\
\hline 46 & 2644 & 103.01 & 330.90 & .3113 \\
\hline 47 & 2645 & 133.13 & 368.70 & .3611 \\
\hline 49 & 2647 & 121.96 & 296.60 & .4112 \\
\hline 49 & 2648 & 113.62 & 315.50 & .3601 \\
\hline 50 & 2649 & 161.57 & 406.90 & .3971 \\
\hline 51 & 2651 & 115.34 & 333.70 & .3406 \\
\hline 52 & 2652 & 124.56 & 333.50 & .3735 \\
\hline 53 & 2654 & 117.23 & 326.20 & .3594 \\
\hline 54 & 2656 & 117.54 & 289.40 & .4062 \\
\hline 55 & 2657 & 105.70 & 289,40 & .3553 \\
\hline 56 & 2659 & 104.31 & 272.30 & .3831 \\
\hline 57 & 2660 & 167.99 & 354.80 & .4735 \\
\hline 58 & 2661 & 86.66 & 300,40 & .2005 \\
\hline 59 & 2663 & 107.19 & 331.20 & .3236 \\
\hline 60 & 2664 & 134.29 & 386.80 & .0472 \\
\hline 61 & 2665 & 123.72 & 345.50 & .3581 \\
\hline
\end{tabular}


TABLE A10. (Continued 15)

STRONTIUM-90 IN ALFALFA (CONT.)

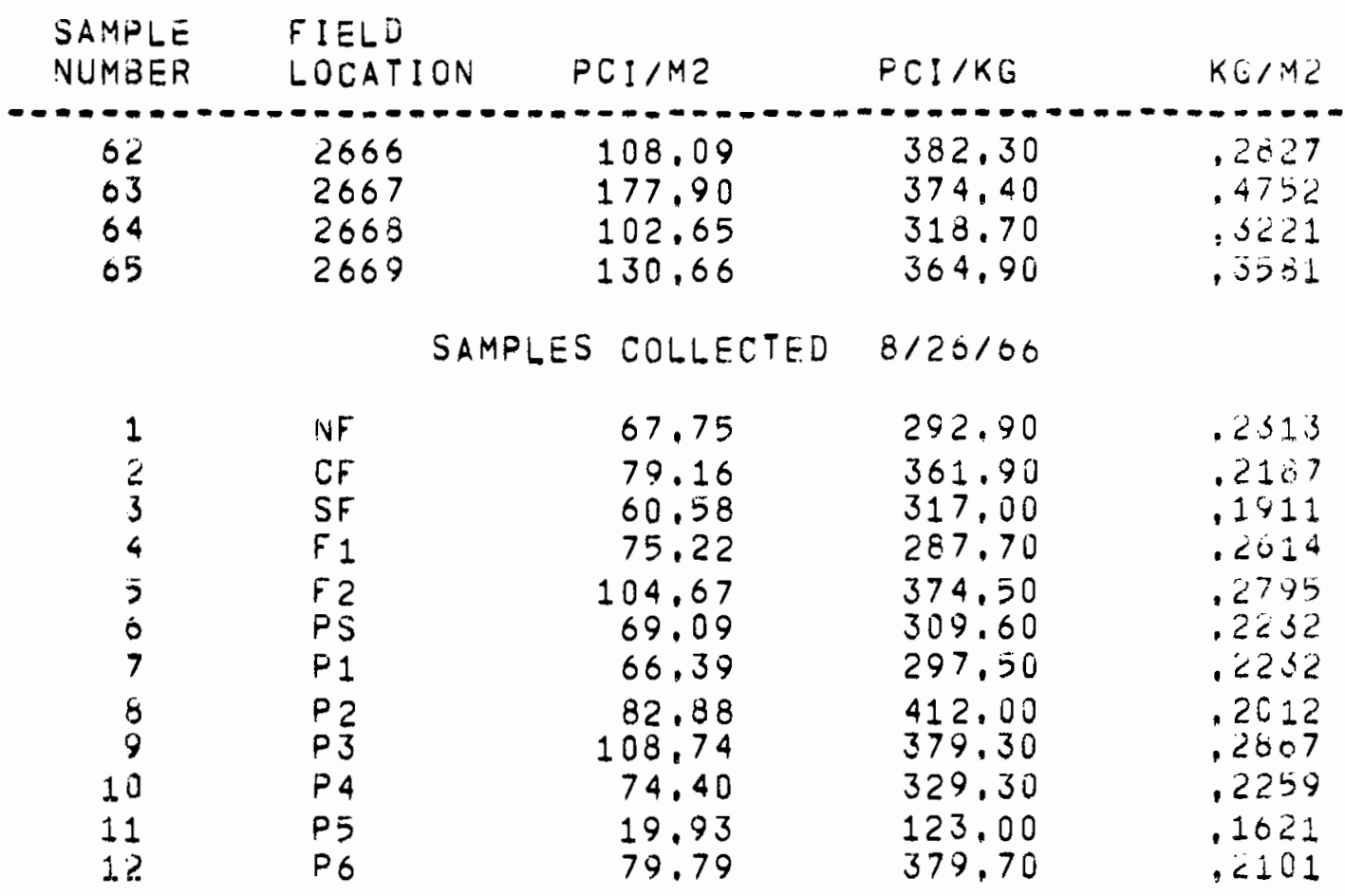

SAMPLES COLLECTED $10 / 13 / 66$

$\begin{array}{rlrll}1 & N F & 66.28 & 298.10 & .2223 \\ 2 & C F & 55.77 & 297.50 & .1874 \\ 3 & S F & 72.70 & 313.80 & .2517 \\ 4 & F 1 & 59.98 & 264.10 & .2271 \\ 5 & F 2 & 67.12 & 284.00 & .2363 \\ 6 & P S & 59.02 & 329.00 & .1794 \\ 7 & F 1 & 57.30 & 290.30 & .1981 \\ 8 & P 2 & 53.18 & 311.40 & .1700 \\ 9 & P 3 & 66.10 & 325.10 & .2033 \\ 10 & P 4 & 58.83 & 312.30 & .1084 \\ 11 & P 5 & 66.58 & 310.50 & .2144 \\ 12 & P 6 & 69.45 & 287.90 & .2412 \\ 13 & 3320 & 72.10 & 332.50 & .2168 \\ 14 & 3321 & 83.78 & 356.50 & .2320 \\ 15 & 3322 & 70.91 & 328.30 & .2160 \\ 16 & 3324 & 83.45 & 383.80 & .2174 \\ 17 & 3326 & 78.50 & 335.40 & .2341 \\ 18 & 3329 & 88.36 & 349.30 & .2530 \\ 19 & 3331 & 89.90 & 383.90 & .2342 \\ 20 & 3334 & 85.17 & 321.20 & .2652 \\ 21 & 3335 & 112.73 & 419.10 & .2690 \\ 22 & 3336 & 92.31 & 308.10 & .2996 \\ 23 & 3338 & 96.17 & 299.60 & .3210 \\ 24 & 3340 & 110.21 & 359.40 & .3067 \\ 25 & 3342 & 54.71 & 333.20 & .1642\end{array}$


TABLE A10. (Continued 16)

STRONTIUM-90 IN ALFALFA (CONT, )

\begin{tabular}{|c|c|c|c|c|}
\hline $\begin{array}{l}\text { SAMPLE } \\
\text { NUMBER }\end{array}$ & $\begin{array}{l}\text { FIELD } \\
\text { LOCATION }\end{array}$ & $P C ! / M 2$ & $P C I / K G$ & $K G / M 2$ \\
\hline$=$ & $--\infty$ & 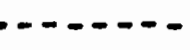 & - & $=--\infty$ \\
\hline 20 & 3344 & 72.86 & 363.90 & .2002 \\
\hline 27 & 3345 & 73.35 & 320.60 & .2288 \\
\hline 28 & 3346 & 80.06 & 375.00 & .2135 \\
\hline 29 & 3347 & 79.80 & 298.40 & .2574 \\
\hline 30 & 3348 & 79.64 & 380.30 & .2094 \\
\hline 31 & 3349 & 95.33 & 339.90 & .2805 \\
\hline 32 & 3350 & 91.69 & 348.00 & .2050 \\
\hline 33 & 3351 & 135.85 & 375.00 & .5594 \\
\hline 34 & 3352 & 39.71 & 403.50 & .2223 \\
\hline 35 & 3353 & 108,89 & 413.30 & .2635 \\
\hline 36 & 3354 & 38.41 & 359.20 & .2461 \\
\hline 37 & 3360 & 62.12 & 394.40 & $.157 b$ \\
\hline 30 & 3361 & 75.17 & 333.10 & .2257 \\
\hline $3 y$ & 3555 & 70.55 & 360.80 & $: 1955$ \\
\hline 40 & 3366 & 72.92 & 339.10 & .2150 \\
\hline 41 & 3367 & 94,36 & 364,60 & .2588 \\
\hline $4 ?$ & 3369 & 66.56 & 354.70 & .1877 \\
\hline 43 & 3370 & 73,11 & 333.30 & .2193 \\
\hline 44 & 3371 & 37.61 & 340.40 &.$\angle 574$ \\
\hline 45 & 3372 & 81.77 & 393.50 & .2077 \\
\hline 46 & 3374 & 74.76 & 374.30 & .1997 \\
\hline 47 & 3376 & 65.03 & 380.40 & .1674 \\
\hline 48 & 3377 & 66.29 & 287.50 & .2306 \\
\hline 49 & 3378 & 52.77 & 318.10 & .1973 \\
\hline 50 & 3379 & 63.42 & 404.80 & .1567 \\
\hline 51 & 3380 & 74.99 & 317.00 & .2366 \\
\hline $5 \ddot{c}$ & 3382 & 57.44 & 314,50 & .2144 \\
\hline 53 & 3384 & 66,91 & 304.70 & .2195 \\
\hline 54 & 3388 & 72,32 & 318.60 & .2270 \\
\hline כi & 3390 & 77.52 & 306.40 & .2595 \\
\hline 50 & 3391 & 34.65 & 291.50 & .2904 \\
\hline 57 & 3393 & 32,66 & 315.00 & .2624 \\
\hline 50 & 3396 & 68.73 & 316.80 & .2170 \\
\hline 59 & 3397 & 91.54 & 336.00 & .2724 \\
\hline $6 \pi$ & 3401 & 90.49 & 395.70 & .2287 \\
\hline 51 & 3402 & 60.01 & 273,60 & .2193 \\
\hline 62 & 3404 & 92.84 & 358.40 & .2591 \\
\hline 63 & 3400 & 73.32 & 360.90 & .1998 \\
\hline 04 & 3409 & 51.68 & 281.90 & .1833 \\
\hline 05 & 3411 & 37.70 & 348,20 & .2219 \\
\hline 66 & 3413 & 75.92 & 364.80 & .2081 \\
\hline 07 & 3414 & 63.63 & 292.00 & .2179 \\
\hline 08 & 3415 & 30.37 & 312.40 & .2573 \\
\hline 67 & 3417 & 79.58 & 356.20 & .2234 \\
\hline 70 & 3418 & 73.87 & 358.70 & .2059 \\
\hline $7:$ & 3419 & 04.51 & 327. & .1970 \\
\hline
\end{tabular}

A. 58 
TABLE A11. CS-137/SR-90 RATIOS IN ALFALFA SAMPLES

\begin{tabular}{|c|c|c|c|c|c|}
\hline $\begin{array}{l}\text { SAMPLE } \\
\text { NUMBER }\end{array}$ & $\begin{array}{l}\text { FIELD } \\
\text { LOC. }\end{array}$ & $\begin{array}{l}137-C S \\
P C ! / M 2\end{array}$ & $\begin{array}{l}90-S R \\
P C I / M 2\end{array}$ & RATIO & KG/M2 \\
\hline & & SAMPLES & COLLECTED & $6 / 18 / 63$ & \\
\hline $\begin{array}{l}1 \\
2 \\
3 \\
4 \\
5 \\
6\end{array}$ & $\begin{array}{l}\text { NF } \\
\text { CF } \\
\text { SE } \\
\text { P1 } \\
\text { P2 } \\
\text { P4 }\end{array}$ & $\begin{array}{l}527,00 \\
511,20 \\
402,70 \\
396,00 \\
457,20 \\
556,00\end{array}$ & $\begin{array}{l}552,10 \\
483,40 \\
424,80 \\
427,50 \\
461,10 \\
481,20\end{array}$ & $\begin{array}{r}.9545 \\
1,0575 \\
, 9480 \\
19263 \\
.9915 \\
1,1554\end{array}$ & $\begin{array}{l}.4114 \\
.4032 \\
.3348 \\
.3510 \\
.3249 \\
.3528\end{array}$ \\
\hline & & SAMPLES & COLLECTED & $7 / 22 / 63$ & \\
\hline $\begin{array}{l}1 \\
2 \\
3 \\
4 \\
5 \\
6\end{array}$ & $\begin{array}{l}\text { NF } \\
C F \\
\text { SF } \\
P 1 \\
P 2 \\
P 3\end{array}$ & $\begin{array}{l}105,20 \\
206,80 \\
157,20 \\
146,20 \\
176,50 \\
122,70\end{array}$ & $\begin{array}{l}116,80 \\
193,40 \\
124,60 \\
146,20 \\
201,70 \\
145,20\end{array}$ & $\begin{array}{r}.9007 \\
1,0693 \\
1,2610 \\
1.0000 \\
.8751 \\
.8450\end{array}$ & $\begin{array}{l}.2968 \\
.4606 \\
.3429 \\
.3609 \\
.4304 \\
.3227\end{array}$ \\
\hline & & SAMPLES & COLLECTED & $8 / 26 / 63$ & \\
\hline $\begin{array}{l}1 \\
2 \\
3 \\
4 \\
5\end{array}$ & $\begin{array}{l}\text { NF } \\
C F \\
P 1 \\
P 2 \\
P 3\end{array}$ & $\begin{array}{l}252,40 \\
202,30 \\
264,70 \\
150,30 \\
104,70\end{array}$ & $\begin{array}{l}142,40 \\
176,20 \\
194,00 \\
128,40 \\
104,40\end{array}$ & $\begin{array}{l}1,7725 \\
1,1481 \\
1,3644 \\
1,1706 \\
1,0029\end{array}$ & $\begin{array}{r}.2744 \\
.2970 \\
.3765 \\
.2790 \\
.2187\end{array}$ \\
\hline & & SAMPLES & COLLECTED & $10 / 6 / 63$ & \\
\hline $\begin{array}{l}1 \\
2 \\
3 \\
4 \\
5 \\
6 \\
7\end{array}$ & $\begin{array}{l}\text { AF } \\
S F \\
P 1 \\
P 2 \\
P 3 \\
169 \\
172\end{array}$ & $\begin{array}{r}69,20 \\
74,80 \\
105,90 \\
50,40 \\
85,90 \\
116,70 \\
83,80\end{array}$ & $\begin{array}{r}55,20 \\
57,80 \\
85,30 \\
49,60 \\
72,80 \\
141,50 \\
98,10\end{array}$ & $\begin{array}{r}1,2536 \\
1,2941 \\
1,2415 \\
1.0161 \\
1,1799 \\
.8247 \\
.8542\end{array}$ & $\begin{array}{l}.0820 \\
.0797 \\
.1043 \\
.0713 \\
.1178 \\
.1917 \\
.1396\end{array}$ \\
\hline & & SAMPLES & COLLECTED & $5 / 14 / 64$ & \\
\hline $\begin{array}{l}1 \\
2 \\
3 \\
4 \\
5 \\
6\end{array}$ & $\begin{array}{l}P 2 \\
187 \\
188 \\
189 \\
190 \\
191\end{array}$ & $\begin{array}{r}957,10 \\
1360,80 \\
1420,80 \\
1952,00 \\
1450,20 \\
1533,90\end{array}$ & $\begin{array}{r}815,20 \\
1093,40 \\
1183,60 \\
1396,20 \\
1218,60 \\
1187,10\end{array}$ & $\begin{array}{l}1.1741 \\
1.2446 \\
1.2004 \\
1.3981 \\
1.1901 \\
1.2921\end{array}$ & $\begin{array}{l}.3160 \\
.4172 \\
.4400 \\
.5784 \\
.4621 \\
.5319\end{array}$ \\
\hline
\end{tabular}


TABLE A11. (Continued 2)

$$
\text { CS-137/SR-90 RATIOS (CONT,) }
$$

\begin{tabular}{|c|c|c|c|c|c|}
\hline $\begin{array}{l}\text { SAMPLE } \\
\text { NUMBER }\end{array}$ & $\begin{array}{l}\text { F IELD } \\
\text { LOC. }\end{array}$ & $\begin{array}{l}137-C S \\
P C I / M 2\end{array}$ & $\begin{array}{l}90-5 R \\
P C \backslash / M 2\end{array}$ & RATIO & $K G / M 2$ \\
\hline & & SAMPLES & COLLECTED & $6 / 16 / 64$ & \\
\hline $\begin{array}{l}1 \\
2 \\
3 \\
4\end{array}$ & $\begin{array}{l}\text { CF } \\
\text { SF } \\
R 2 \\
\text { R3 }\end{array}$ & $\begin{array}{l}233,80 \\
260,60 \\
246.30 \\
256.30\end{array}$ & $\begin{array}{l}362,00 \\
293,20 \\
348,80 \\
302,20\end{array}$ & $\begin{array}{l}.6459 \\
.8888 \\
.7061 \\
.8481\end{array}$ & $\begin{array}{l}.2625 \\
.2604 \\
.2936 \\
.2630\end{array}$ \\
\hline & & SAMPLES & COLLECTED & $7 / 20 / 64$ & \\
\hline $\begin{array}{l}1 \\
2 \\
3 \\
4 \\
5\end{array}$ & $\begin{array}{l}\text { NF } \\
C F \\
\text { SF } \\
R 1 \\
\text { P3 }\end{array}$ & $\begin{array}{r}102,10 \\
60,90 \\
63,40 \\
66,30 \\
40,40\end{array}$ & $\begin{array}{r}89,50 \\
106,20 \\
103,60 \\
102,80 \\
76,40\end{array}$ & $\begin{array}{r}1.1408 \\
.5734 \\
.6120 \\
.6449 \\
.5288\end{array}$ & $\begin{array}{l}.2333 \\
.2514 \\
.2930 \\
.2471 \\
.1839\end{array}$ \\
\hline & & SAMPLES & COLLECTED & $8 / 25 / 64$ & \\
\hline $\begin{array}{l}1 \\
2 \\
3 \\
4\end{array}$ & $\begin{array}{l}P 1 \\
P 2 \\
P 3 \\
P 4\end{array}$ & $\begin{array}{l}62,80 \\
73,40 \\
56,30 \\
38,80\end{array}$ & $\begin{array}{r}95,10 \\
106,80 \\
90,90 \\
70,20\end{array}$ & $\begin{array}{l}.6604 \\
.6873 \\
.6194 \\
.5527\end{array}$ & $\begin{array}{l}.2592 \\
.2931 \\
.2397 \\
.1960\end{array}$ \\
\hline & & SAMPLES & COLLECTED & $10 / 18 / 64$ & \\
\hline $\begin{array}{l}1 \\
2 \\
3 \\
4 \\
5 \\
6 \\
7 \\
8 \\
9 \\
10 \\
11 \\
12 \\
13 \\
14 \\
15 \\
16 \\
17 \\
18 \\
19 \\
20 \\
21 \\
22\end{array}$ & $\begin{array}{l}\text { NF } \\
C F \\
P 2 \\
P A \\
581 \\
582 \\
583 \\
584 \\
585 \\
587 \\
588 \\
589 \\
591 \\
593 \\
594 \\
595 \\
596 \\
597 \\
598 \\
599 \\
600 \\
602\end{array}$ & $\begin{array}{l}30,40 \\
31,30 \\
29,40 \\
30,20 \\
28,90 \\
25,40 \\
30,00 \\
25,90 \\
33,60 \\
47,20 \\
36,60 \\
10,00 \\
14,10 \\
44,20 \\
41,40 \\
24,90 \\
55,20 \\
40,40 \\
30,00 \\
30,70 \\
26,60 \\
43,60\end{array}$ & $\begin{array}{l}54,10 \\
94,00 \\
63,70 \\
67,40 \\
74,00 \\
65,20 \\
57,90 \\
64,30 \\
69,30 \\
68,50 \\
54,20 \\
40,50 \\
39,20 \\
86,70 \\
82,60 \\
55,90 \\
87,80 \\
85,00 \\
79,00 \\
70,40 \\
60,40 \\
74,20\end{array}$ & $\begin{array}{l}.5619 \\
.3330 \\
.4615 \\
.4481 \\
.3905 \\
.3896 \\
.5181 \\
.4028 \\
.4848 \\
.6891 \\
.6753 \\
.2469 \\
.3597 \\
.5098 \\
.5012 \\
.4454 \\
.6287 \\
.4753 \\
.3797 \\
.4361 \\
.4404 \\
.5876\end{array}$ & $\begin{array}{l}.1490 \\
.1337 \\
.1247 \\
.1420 \\
.1591 \\
.1400 \\
.1233 \\
.1670 \\
.1583 \\
.1777 \\
.1264 \\
.1232 \\
.1100 \\
.2104 \\
.2147 \\
.1326 \\
.2073 \\
.2119 \\
.1865 \\
.1609 \\
.1448 \\
.1253\end{array}$ \\
\hline
\end{tabular}


TABLE A11. (Continued 3)

CS-137/SR-90 RATIOS (CONT,)

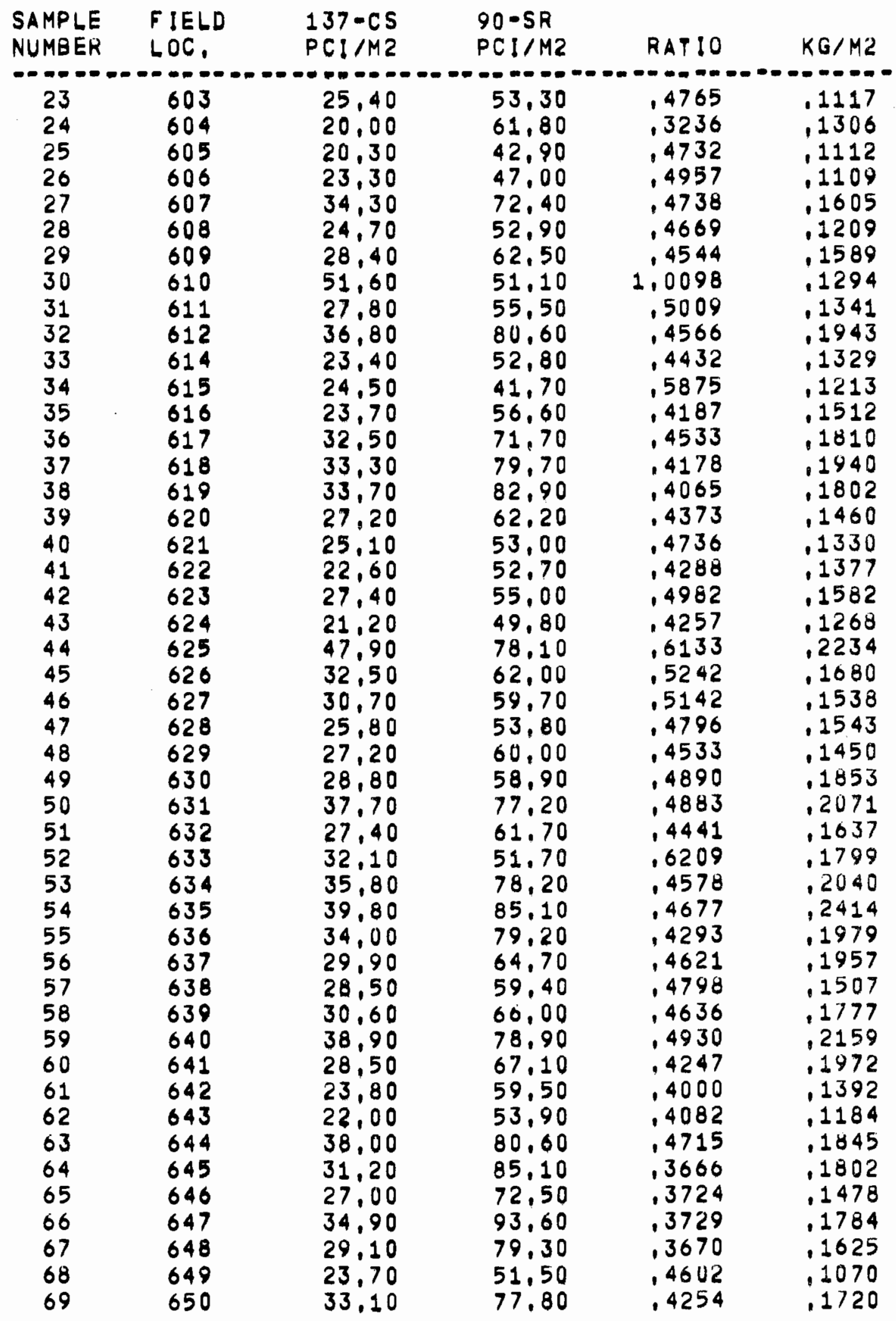


TABLE A11. (Continued 4)

CS-137/SR-90 RATIOS (CONT,)

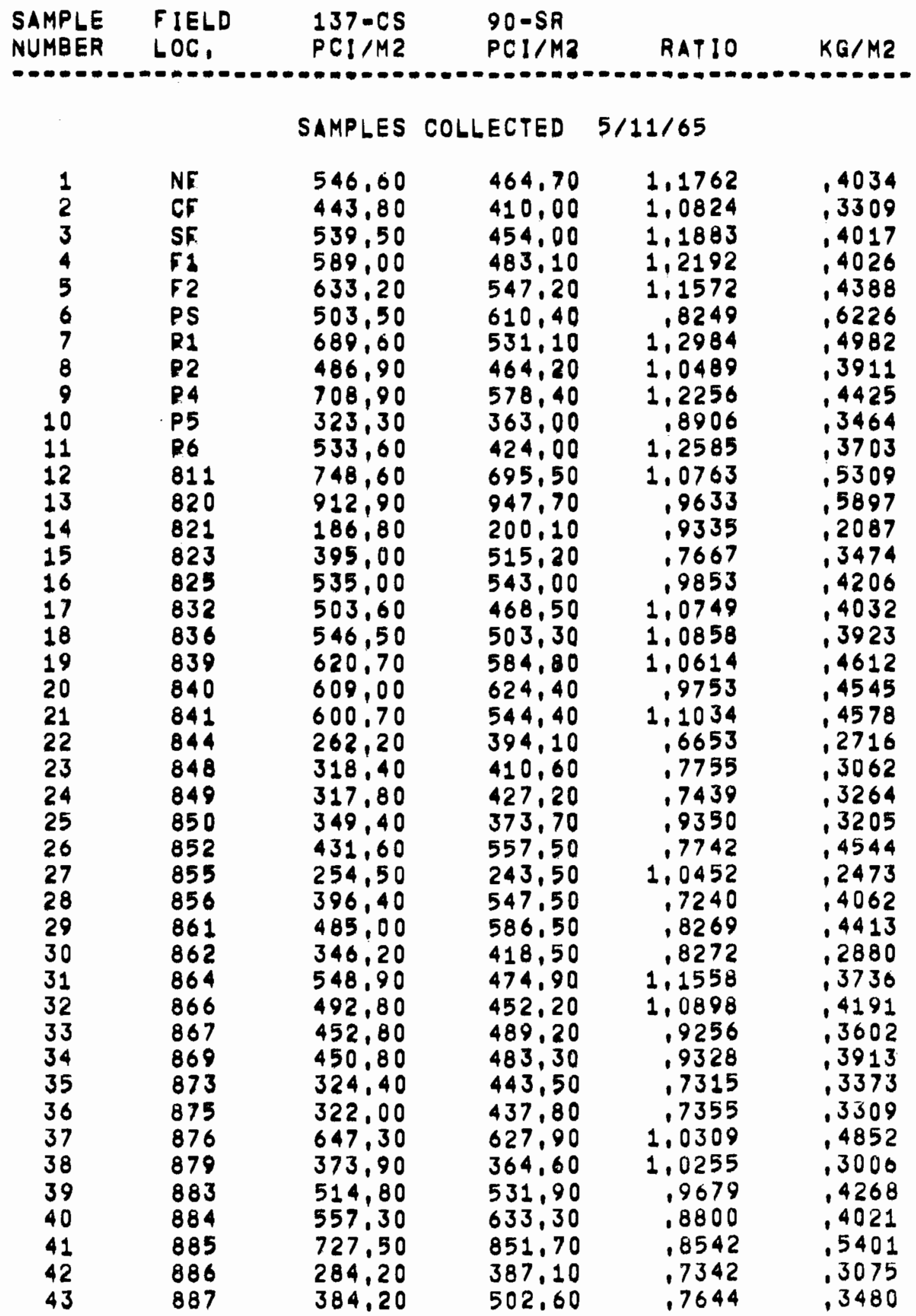


TABLE A11. (Continued 5)

CS-137/SR-90 RATIOS (CONT,)

$\begin{array}{llllll}\text { SAMPLE } & \text { FIILD } & 137-C S & 90-S R & & \\ \text { NUMBER } & \text { LOC, } & \text { PCI/M2 } & \text { PCI/M2 } & \text { RATIO } & \mathrm{KG} / \mathrm{ML}\end{array}$

SAMPLES COLLECTED 6/16/65

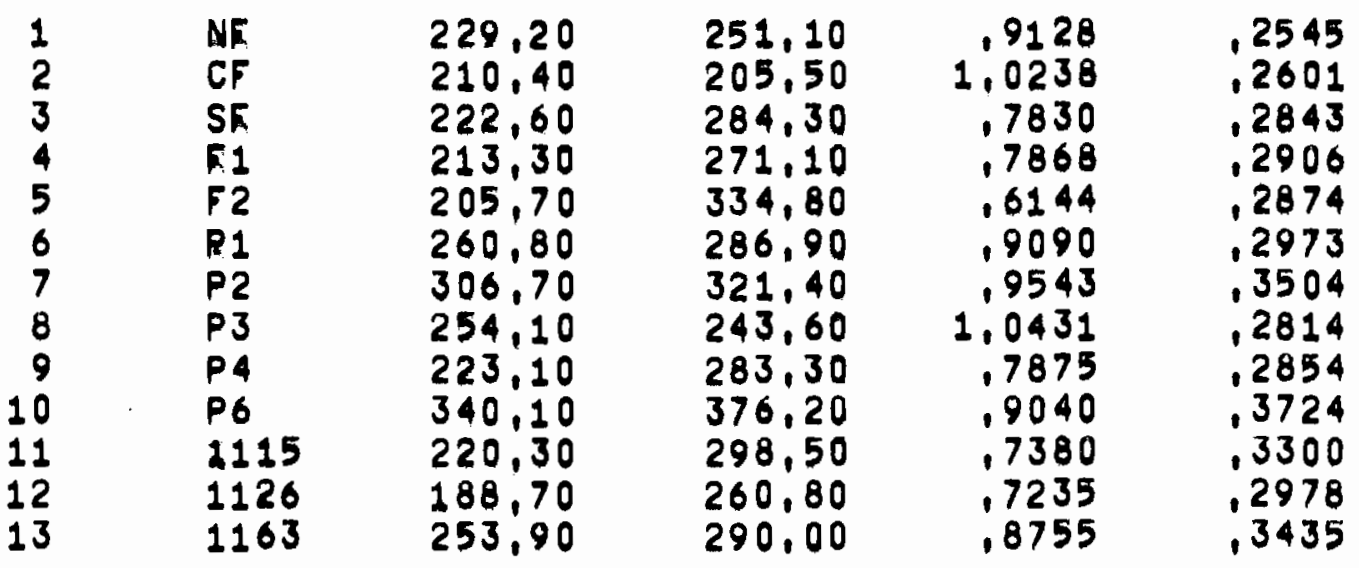

\section{SAMPLES COLLECTED $\quad 7 / 20 / 65$}

\begin{tabular}{|c|c|c|c|c|c|}
\hline $\begin{array}{l}1 \\
2 \\
3 \\
4 \\
5 \\
6 \\
7 \\
8 \\
9 \\
10 \\
11 \\
12 \\
13 \\
14 \\
15 \\
16 \\
17 \\
18 \\
19 \\
20 \\
21 \\
22 \\
23 \\
24 \\
25 \\
26 \\
27 \\
28\end{array}$ & $\begin{array}{l}N F \\
S F \\
P S \\
P 1 \\
P 2 \\
P 3 \\
P 4 \\
P 5 \\
P 6 \\
1380 \\
1382 \\
1383 \\
1384 \\
1385 \\
1386 \\
1387 \\
1389 \\
1390 \\
1391 \\
1392 \\
1393 \\
1394 \\
1396 \\
1397 \\
1398 \\
1400 \\
1403 \\
1404\end{array}$ & $\begin{array}{l}35,70 \\
44,00 \\
45,00 \\
40,80 \\
40,90 \\
32,40 \\
33,50 \\
39,70 \\
42,80 \\
43,80 \\
31,80 \\
41,80 \\
41,90 \\
36,100 \\
26,50 \\
36,50 \\
23,00 \\
32,00 \\
57,70 \\
55,60 \\
52,40 \\
34,00 \\
31,20 \\
29,70 \\
44,50 \\
28,10 \\
42,60 \\
37,10\end{array}$ & $\begin{array}{r}87,50 \\
93,10 \\
140,10 \\
103,20 \\
121,20 \\
101,20 \\
109,50 \\
118,60 \\
106,80 \\
121,60 \\
82,40 \\
105,40 \\
93,10 \\
92,90 \\
75,60 \\
107,90 \\
69,10 \\
85,90 \\
124,70 \\
141,80 \\
120,40 \\
105,10 \\
96,50 \\
84,40 \\
116,20 \\
101,30 \\
138,40 \\
111,40\end{array}$ & $\begin{array}{l}.4080 \\
.4726 \\
.3212 \\
.3953 \\
.3375 \\
.3202 \\
.3059 \\
.3347 \\
.4007 \\
.3602 \\
.3859 \\
.3966 \\
.4501 \\
.3886 \\
.3505 \\
.3568 \\
.3329 \\
.3725 \\
.4627 \\
.3921 \\
.4352 \\
.3235 \\
.3233 \\
.3519 \\
.3830 \\
.2774 \\
.3078 \\
.3330\end{array}$ & $\begin{array}{l}.2817 \\
.3107 \\
.3882 \\
.3403 \\
.3525 \\
.3076 \\
.3067 \\
.3320 \\
.3620 \\
.2989 \\
.2201 \\
.2709 \\
.2980 \\
.2436 \\
.2098 \\
.2574 \\
.1429 \\
.2181 \\
.3232 \\
.3819 \\
.3125 \\
.2660 \\
.2679 \\
.2222 \\
.3238 \\
.2451 \\
.3172 \\
.3123\end{array}$ \\
\hline
\end{tabular}


TABLE A17. (Continued 6)

CS-137/SR-90 RATIOS (CONT,)

\begin{tabular}{|c|c|c|c|c|c|}
\hline $\begin{array}{l}\text { SAMPLE } \\
\text { NUMBER }\end{array}$ & $\begin{array}{l}\text { F IELD } \\
\text { LOC. }\end{array}$ & $\begin{array}{l}137-C S \\
P C I / M 2\end{array}$ & $\begin{array}{l}90-S R \\
P C ! / M 2\end{array}$ & RATIU & $K G / M Z$ \\
\hline-- & -- & $=-\infty \cdot$ & $\ldots$ & 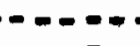 & $\cdots$ \\
\hline 29 & 1412 & 25.90 & 94,80 & .2732 & .2442 \\
\hline 30 & 1413 & 24.20 & 97.80 & .2474 & .2324 \\
\hline 31 & 1414 & 28,10 & 107,30 & .2619 & .2733 \\
\hline 32 & 1416 & 33.00 & 126,80 & .2650 & .2805 \\
\hline 33 & 1417 & 41,00 & 113,80 & .3650 & 3131 \\
\hline 34 & 1421 & 42,90 & 125.50 & .3418 & .3478 \\
\hline 35 & 1424 & 32.00 & 104,80 & 3111 & .2030 \\
\hline 36 & 1434 & 44.20 & 161,00 & .2745 & .3714 \\
\hline 37 & 1444 & 35,80 & 114,40 &, 3129 & .2840 \\
\hline 38 & 1446 & 40.10 & 131,40 &, 3052 & 3232 \\
\hline 39 & 1448 & 44,00 & 170,60 & .2614 & .3556 \\
\hline 40 & 1415 & 34,50 & 108.40 & .3183 & 3191 \\
\hline 41 & 1419 & 48.50 & 125.20 & .3874 & .3766 \\
\hline 42 & 1423 & 29,00 & 133,50 & .2172 & .3106 \\
\hline 43 & 1426 & 31,60 & 93.40 & .3383 & .2763 \\
\hline 44 & 1431 & 39.30 & 114,80 & 3423 & 3044 \\
\hline 45 & 1433 & 44,40 & 125,70 & 3532 & 3797 \\
\hline 46 & 1438 & 55.80 & 134,20 & .4158 & .4054 \\
\hline 47 & 1439 & 29,90 & 101,40 & .2949 & .2735 \\
\hline 48 & 1441 & 47.40 & 143,90 & .3294 & .3859 \\
\hline 49 & 1442 & 30,90 & 100,00 & .3090 & .2708 \\
\hline 50 & 1443 & 41,90 & 128,20 & 3268 & .3428 \\
\hline 51 & 1445 & 40,80 & 146,60 & .2783 & .3557 \\
\hline 52 & 1447 & 23,40 & 112,50 & .2080 &, 2471 \\
\hline 53 & 1449 & 27.40 & 71.80 & .3810 & 1908 \\
\hline
\end{tabular}

SAMPLES COLLECTED $8 / 26 / 65$

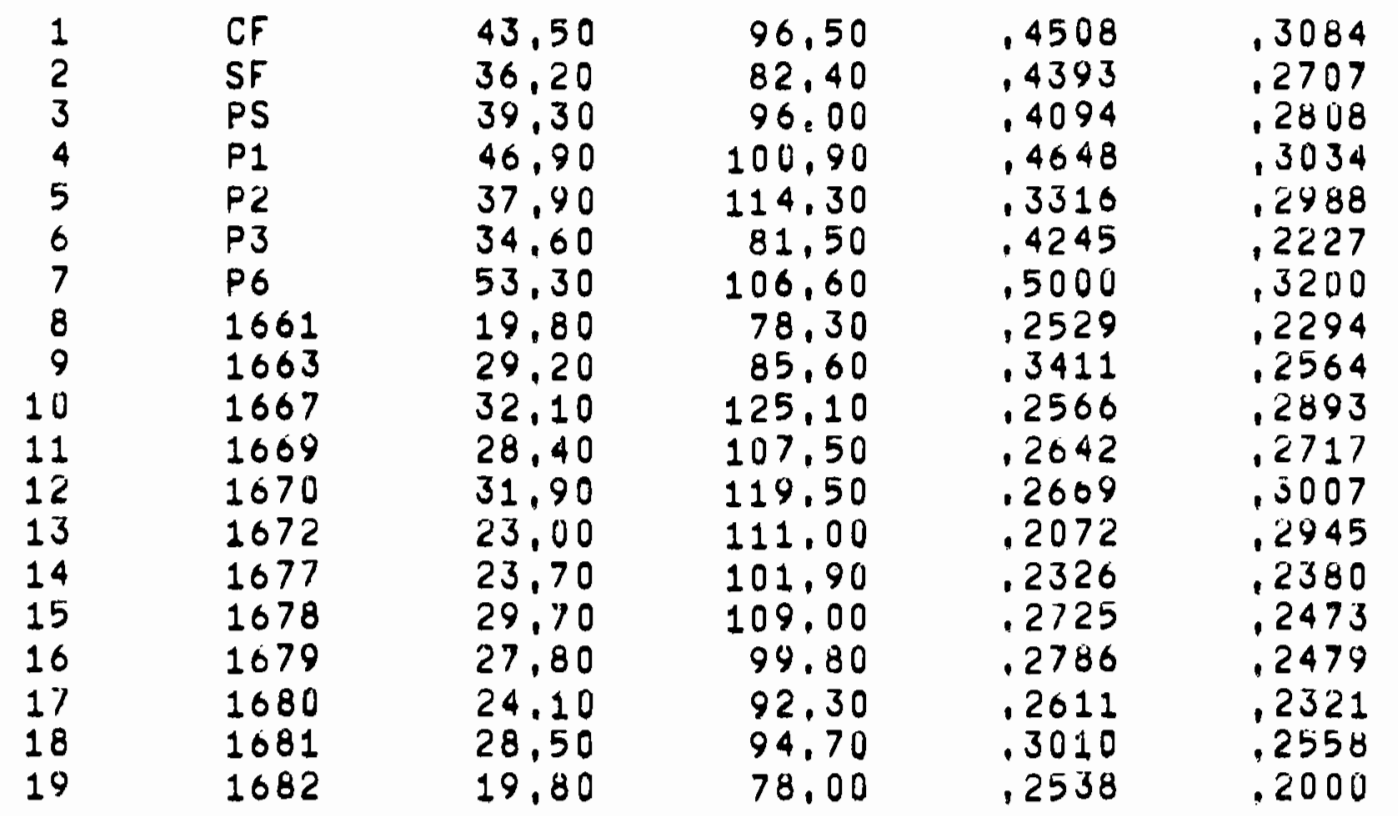


TABLE A11. (Continued 7)

CS-137/SR-90 RATIOS (CONT,)

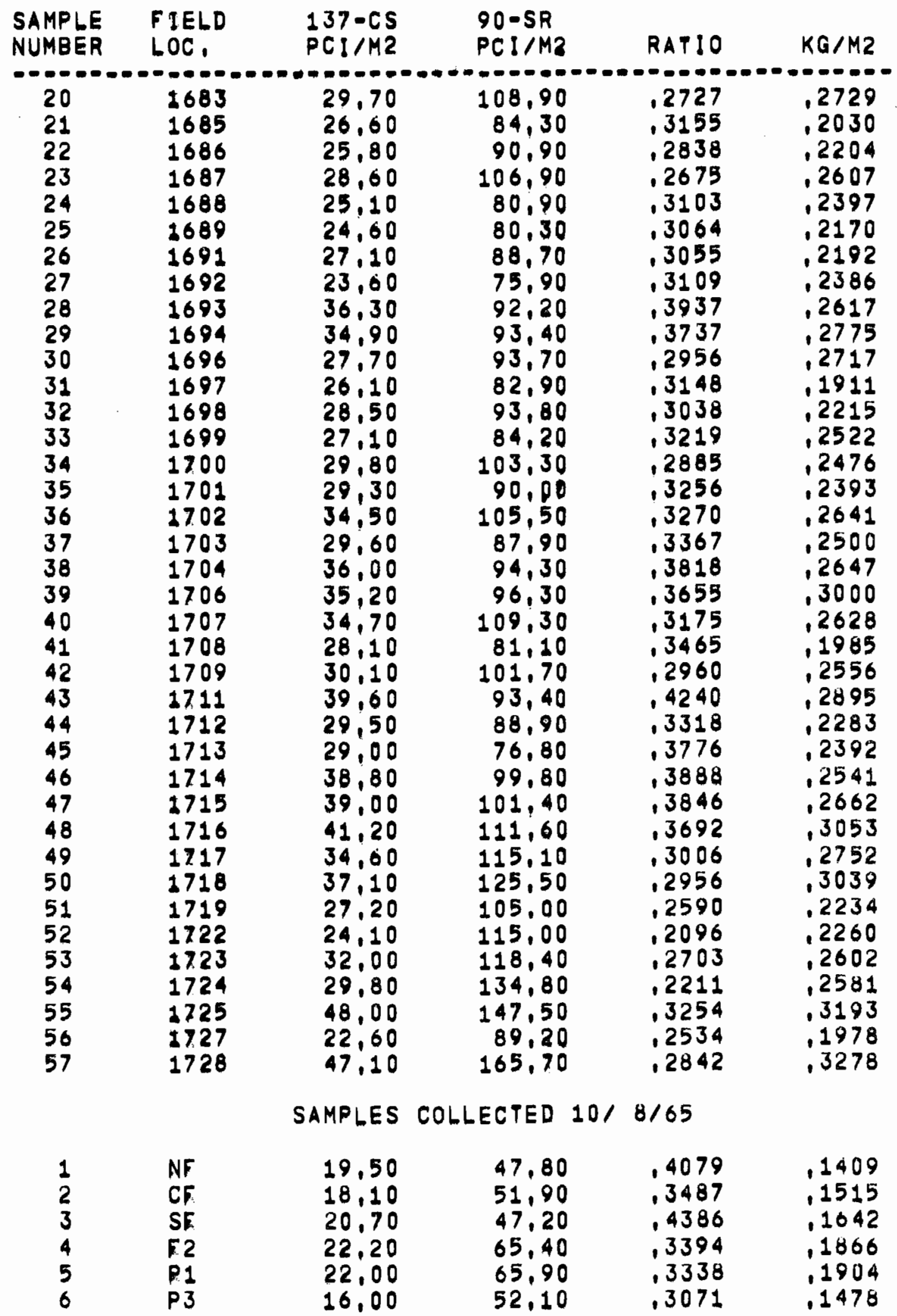


TABLE A11. (Continued 8)

CS-137/SR-9O RATIOS (CONT,)

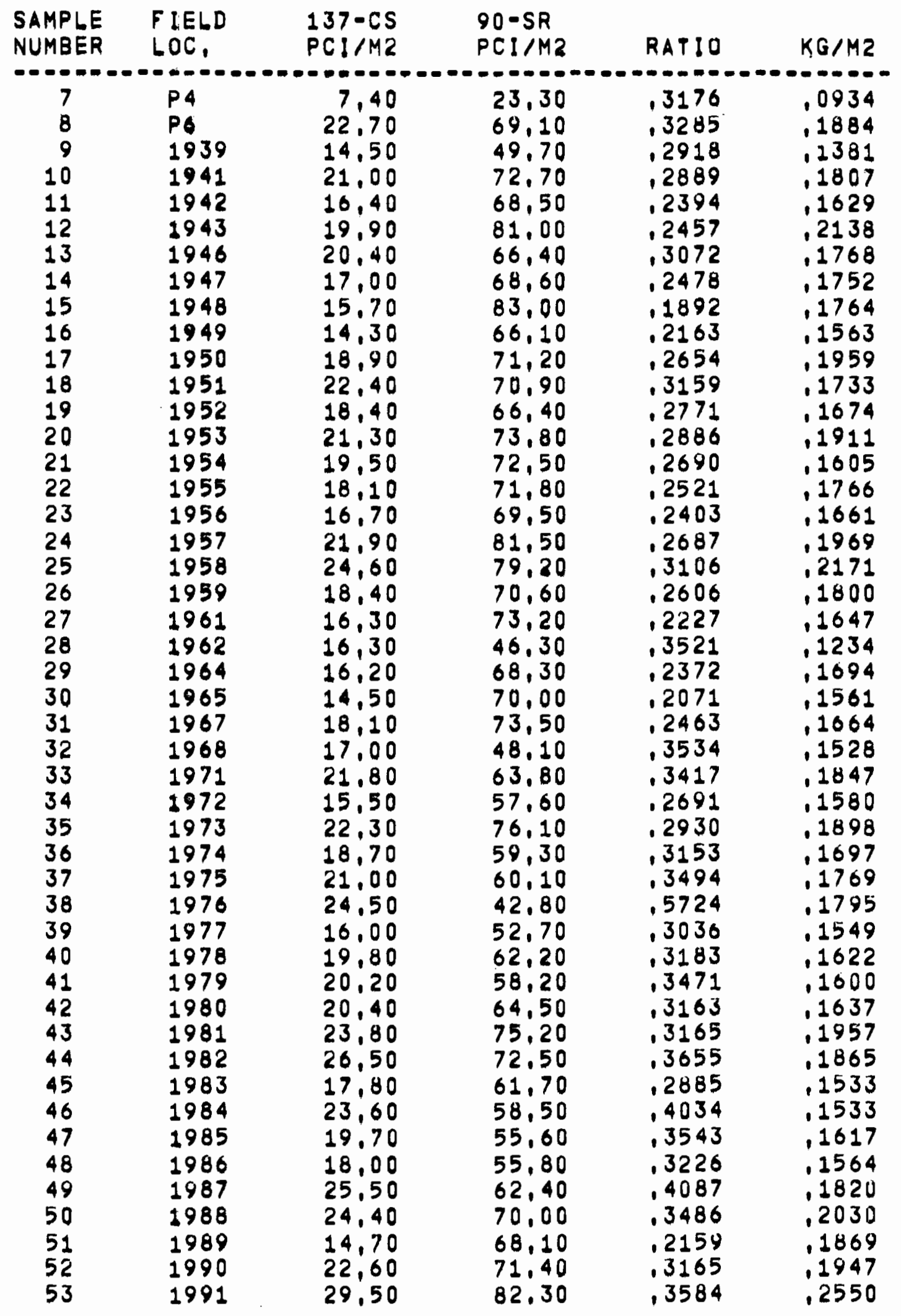


TABLE A11. (Continued 9)

CS.137/SR=90 RATIOS (CONT,)

\begin{tabular}{|c|c|c|c|c|c|}
\hline $\begin{array}{l}\text { SAMPLE } \\
\text { NUMBER }\end{array}$ & $\begin{array}{l}\text { FIELD } \\
\text { LOC. }\end{array}$ & $\begin{array}{l}137-C S \\
P C ! / M 2\end{array}$ & $\begin{array}{l}90-S R \\
P C I / M Z\end{array}$ & RATIO & $K G / M 2$ \\
\hline \multirow[t]{2}{*}{$\begin{array}{l}54 \\
55 \\
56 \\
57 \\
58 \\
59 \\
60 \\
61 \\
62 \\
63 \\
64 \\
65 \\
66 \\
67 \\
68\end{array}$} & $\begin{array}{l}1992 \\
1993 \\
1994 \\
1995 \\
1996 \\
1997 \\
1999 \\
2000 \\
2001 \\
2002 \\
2003 \\
2005 \\
2006 \\
2007 \\
2008\end{array}$ & $\begin{array}{l}25,30 \\
27,70 \\
23,10 \\
24,40 \\
23,90 \\
17,10 \\
24,40 \\
20,50 \\
25,00 \\
16,80 \\
24,90 \\
24,60 \\
17,80 \\
19,50 \\
23,70\end{array}$ & $\begin{array}{l}82,90 \\
74,30 \\
74,30 \\
74,70 \\
70,00 \\
74,20 \\
68,00 \\
71,50 \\
67.80 \\
60,20 \\
74,50 \\
87,00 \\
60,10 \\
78,00 \\
94,40\end{array}$ & $\begin{array}{l}.3052 \\
.3728 \\
.3109 \\
.3266 \\
.3414 \\
.2305 \\
.3588 \\
.2867 \\
.3687 \\
.2791 \\
.3342 \\
.2828 \\
.2962 \\
.2500 \\
.2511\end{array}$ & $\begin{array}{l}.2250 \\
.2019 \\
.1952 \\
.2068 \\
.1885 \\
.1790 \\
.1928 \\
.1719 \\
.2018 \\
.1432 \\
.1782 \\
.2231 \\
.1355 \\
.1707 \\
.2173\end{array}$ \\
\hline & & SAMPLES & COLLECTED & $5 / 11 / 66$ & \\
\hline \multirow[t]{2}{*}{$\begin{array}{r}1 \\
2 \\
3 \\
4 \\
5 \\
6 \\
7 \\
8 \\
9 \\
10 \\
11 \\
12 \\
13 \\
14\end{array}$} & $\begin{array}{l}\text { NF } \\
C F \\
F 1 \\
F 2 \\
P S \\
P 1 \\
P 2 \\
P 3 \\
P 4 \\
P 6 \\
2348 \\
2351 \\
2368 \\
2389\end{array}$ & $\begin{array}{l}249,30 \\
337.90 \\
224.20 \\
221.70 \\
203,00 \\
298,40 \\
286,00 \\
258,00 \\
278,80 \\
319,80 \\
232.40 \\
245,70 \\
222,70 \\
286,80\end{array}$ & $\begin{array}{l}350,60 \\
451,10 \\
246,80 \\
292,50 \\
270,00 \\
359,50 \\
352,10 \\
308,60 \\
319,50 \\
396,10 \\
374,10 \\
495,20 \\
466,00 \\
463,10\end{array}$ & $\begin{array}{l}.7111 \\
.7491 \\
.9084 \\
.7579 \\
.7519 \\
.8300 \\
.8123 \\
.8360 \\
.8726 \\
.8074 \\
.6212 \\
.4962 \\
.4779 \\
.6193\end{array}$ & $\begin{array}{r}.4509 \\
.5255 \\
.3897 \\
.3899 \\
.3899 \\
.4489 \\
.4489 \\
.4253 \\
.4632 \\
.5279 \\
.4154 \\
.5938 \\
.6138 \\
.6035\end{array}$ \\
\hline & & SAMPLES & COLLECTED & $6 / 16 / 66$ & \\
\hline $\begin{array}{r}1 \\
2 \\
3 \\
4 \\
5 \\
6 \\
7 \\
8 \\
9 \\
10 \\
11 \\
12\end{array}$ & $\begin{array}{l}N F \\
C F \\
\text { SF } \\
71 \\
F 2 \\
P S \\
P 1 \\
P 2 \\
P 3 \\
P 4 \\
P 5 \\
P 6\end{array}$ & $\begin{array}{l}35,80 \\
32,70 \\
33,80 \\
29,70 \\
28,40 \\
39,20 \\
24,90 \\
31,70 \\
36,10 \\
22,80 \\
34,30 \\
21,20\end{array}$ & $\begin{array}{r}106,00 \\
115,80 \\
101,70 \\
85,80 \\
93,20 \\
149,80 \\
76,70 \\
99,50 \\
113,00 \\
76,30 \\
114,70 \\
64,70\end{array}$ & $\begin{array}{r}.3377 \\
.2824 \\
.3324 \\
.3462 \\
.3047 \\
.2617 \\
.3246 \\
.3186 \\
.3195 \\
.2988 \\
.2990 \\
.3277\end{array}$ & $\begin{array}{l}.3344 \\
.3218 \\
.3425 \\
.2635 \\
.3058 \\
.4242 \\
.2449 \\
.2973 \\
.3330 \\
.2636 \\
.3147 \\
.2302\end{array}$ \\
\hline
\end{tabular}


TABLE All. (Continued 10)

CS=137/SR-90 RATIOS (CONT,)

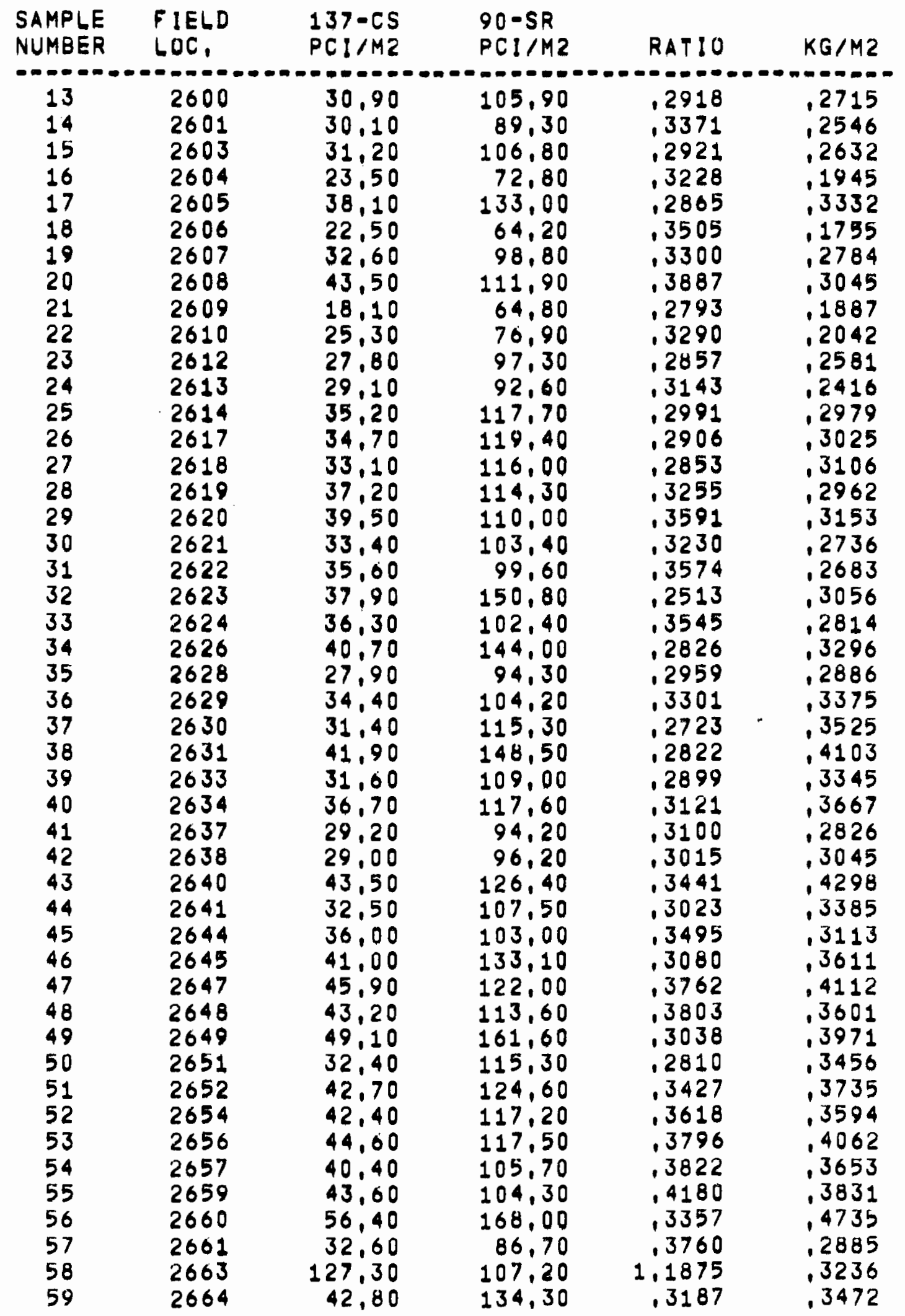


TABLE A11. (Continued 11)

CS-137/SR=9O RATIOS (CONT,)

\begin{tabular}{|c|c|c|c|c|c|}
\hline $\begin{array}{l}\text { SAMPLE } \\
\text { NUMBER }\end{array}$ & $\begin{array}{l}\text { F IELD } \\
\text { LOC, }\end{array}$ & $\begin{array}{l}137-C S \\
P C ! / M 2\end{array}$ & $\begin{array}{l}90-\mathrm{SR} \\
\mathrm{PCl} / \mathrm{M} 2\end{array}$ & RATIO & $K G / M 2$ \\
\hline \multirow[t]{2}{*}{$\begin{array}{l}60 \\
61 \\
62 \\
63 \\
64\end{array}$} & $\begin{array}{l}2665 \\
2666 \\
2667 \\
2668 \\
2669\end{array}$ & $\begin{array}{l}52,30 \\
33,30 \\
58,90 \\
68,30 \\
77,00\end{array}$ & $\begin{array}{l}123,70 \\
108,10 \\
177,90 \\
102,60 \\
130,70\end{array}$ & $\begin{array}{r}.4228 \\
.3080 \\
.3311 \\
.6657 \\
.5891\end{array}$ & $\begin{array}{r}.3581 \\
.2827 \\
.4752 \\
.3221 \\
.3581\end{array}$ \\
\hline & & SAMPLES & COLLECTED & $8 / 26 / 66$ & \\
\hline \multirow[t]{2}{*}{$\begin{array}{l}1 \\
2 \\
3 \\
4 \\
5 \\
6 \\
7\end{array}$} & $\begin{array}{l}\text { NF } \\
\text { SF } \\
\text { F1 } \\
\text { F2 } \\
\text { P3 } \\
\text { R4 } \\
\text { P5 }\end{array}$ & $\begin{array}{l}14,00 \\
14,60 \\
46,30 \\
38,00 \\
93,10 \\
15,20 \\
12,40\end{array}$ & $\begin{array}{r}67,70 \\
60,60 \\
75,20 \\
104,70 \\
108,70 \\
74,40 \\
19,90\end{array}$ & $\begin{array}{l}.2068 \\
.2409 \\
.6157 \\
.3629 \\
.8565 \\
.2043 \\
.6231\end{array}$ & $\begin{array}{l}.2313 \\
.1911 \\
.2614 \\
.2795 \\
.2867 \\
.2259 \\
.1621\end{array}$ \\
\hline & & SAMPLES & COLLECTED & $10 / 13 / 66$ & \\
\hline $\begin{array}{l}1 \\
2 \\
3 \\
4 \\
5 \\
6 \\
7 \\
8 \\
9 \\
10 \\
11 \\
12 \\
13 \\
14 \\
15 \\
16 \\
17 \\
18 \\
19 \\
20 \\
21 \\
22 \\
23 \\
24 \\
25 \\
26 \\
27 \\
28 \\
29\end{array}$ & $\begin{array}{l}\text { NF } \\
C F \\
\text { SF } \\
\text { E1 } \\
F 2 \\
P S \\
P 1 \\
P 2 \\
P 3 \\
P 4 \\
P 5 \\
P 6 \\
3320 \\
3321 \\
3322 \\
3324 \\
3326 \\
3329 \\
3334 \\
3335 \\
3336 \\
3338 \\
3340 \\
3342 \\
3344 \\
3345 \\
3346 \\
3547 \\
3348\end{array}$ & $\begin{array}{l}28,10 \\
25,00 \\
27,80 \\
27,70 \\
22,50 \\
49,00 \\
22,00 \\
24,60 \\
22,70 \\
20,20 \\
25,00 \\
24,70 \\
29,90 \\
34,40 \\
26,90 \\
25,00 \\
21,90 \\
24,30 \\
29,60 \\
29,90 \\
32,80 \\
29,20 \\
33,10 \\
19,00 \\
22,40 \\
22,00 \\
18,70 \\
28,20 \\
21,30\end{array}$ & $\begin{array}{r}60,30 \\
55,80 \\
72,70 \\
60,00 \\
67,10 \\
59,00 \\
57,50 \\
53,20 \\
66,10 \\
58,80 \\
66,60 \\
69,50 \\
72,10 \\
83,80 \\
70,90 \\
83,40 \\
78,50 \\
88,40 \\
85,20 \\
112,70 \\
92,30 \\
96,20 \\
110,20 \\
54,70 \\
72,90 \\
73,40 \\
80,10 \\
79,80 \\
79,60\end{array}$ & $\begin{array}{l}.4238 \\
.4480 \\
.3824 \\
.4617 \\
.3353 \\
.8305 \\
.3826 \\
.4624 \\
.3434 \\
.3435 \\
.3754 \\
.3554 \\
.4147 \\
.4105 \\
.3794 \\
.2998 \\
.2790 \\
.2749 \\
.3474 \\
.2653 \\
.3554 \\
.3035 \\
.3004 \\
.3473 \\
.3073 \\
.2997 \\
.2335 \\
.3534 \\
.2676\end{array}$ & $\begin{array}{l}.2223 \\
.1874 \\
.2317 \\
.2271 \\
.2363 \\
.1794 \\
.1981 \\
.1708 \\
.2033 \\
.1884 \\
.2144 \\
.2412 \\
.2168 \\
.2350 \\
.2160 \\
.2174 \\
.2341 \\
.2530 \\
.2652 \\
.2690 \\
.2996 \\
.3210 \\
.3067 \\
.1642 \\
.2002 \\
.2288 \\
.2135 \\
.2674 \\
.2094\end{array}$ \\
\hline
\end{tabular}


TABLE A11. (Continued 12)

CS-137/SRM9O RATIOS (CONT,)

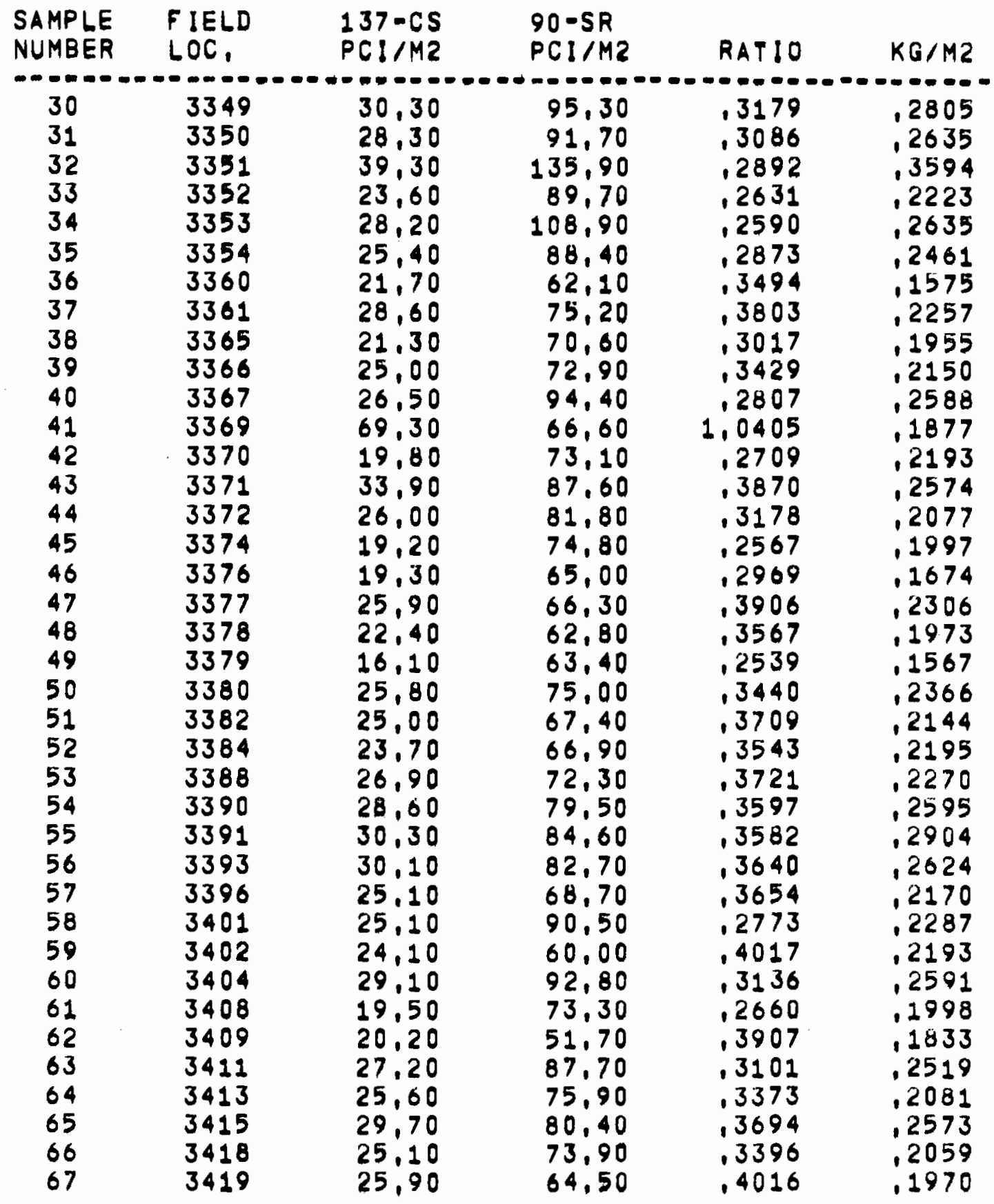




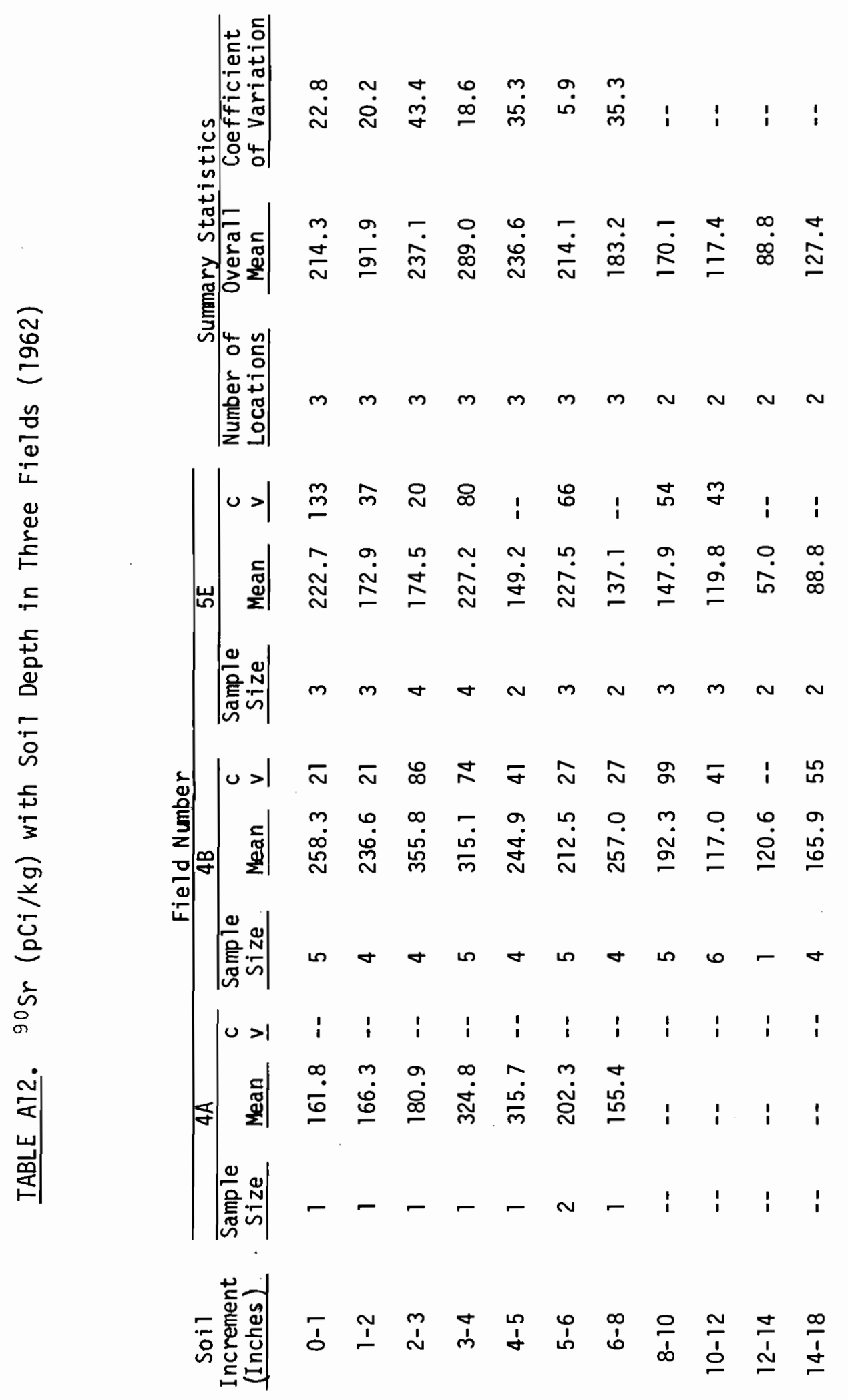




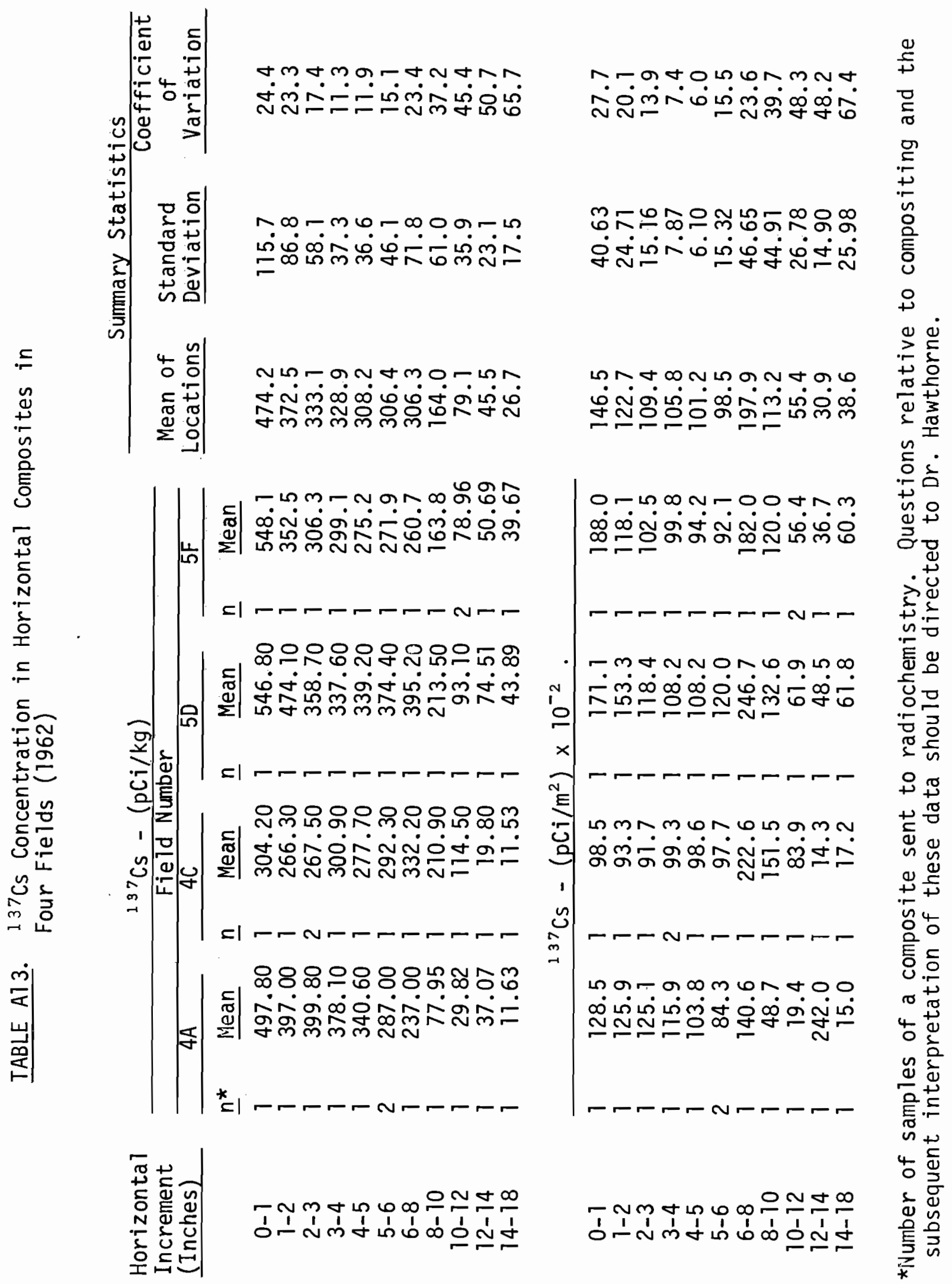




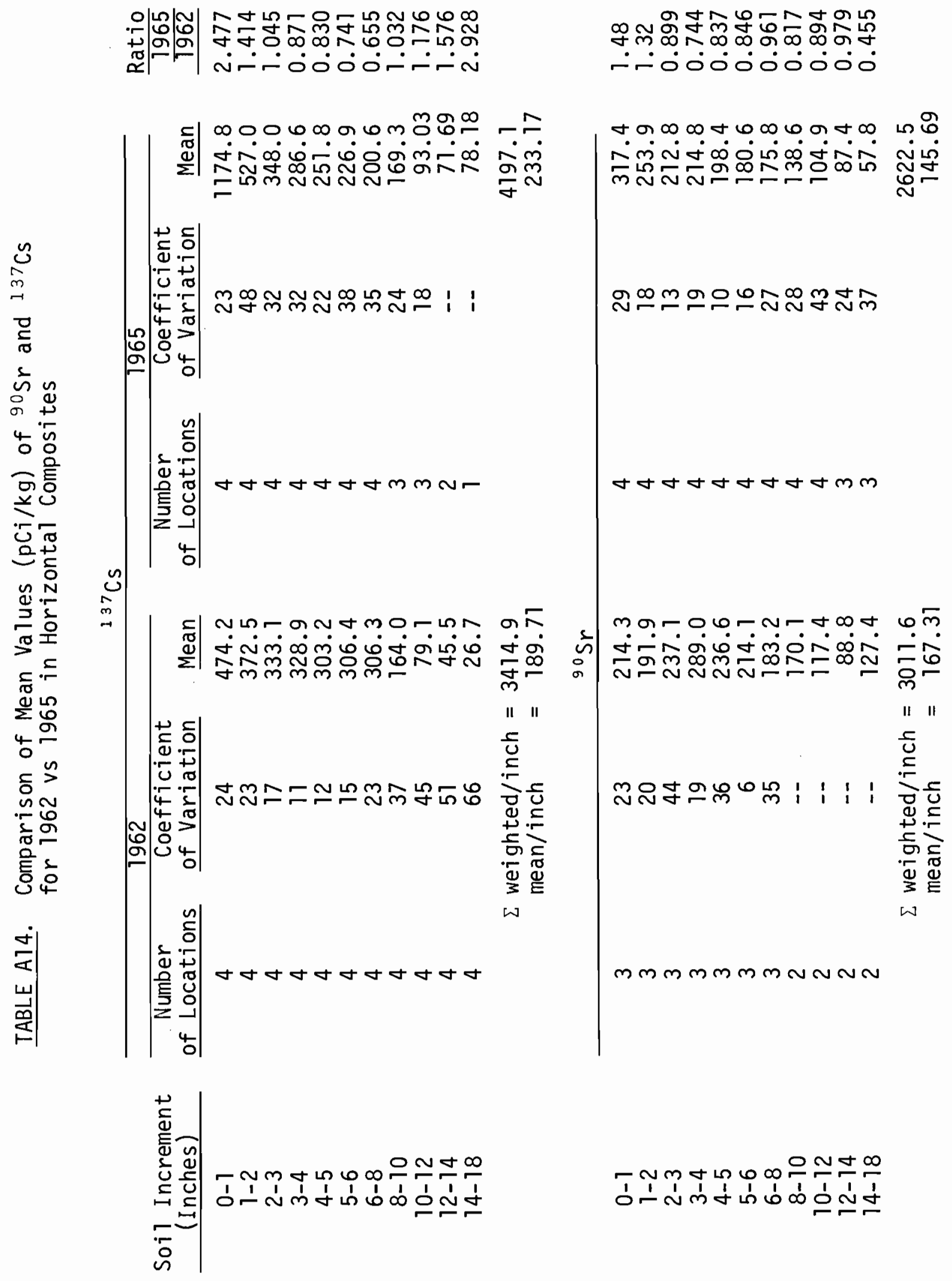




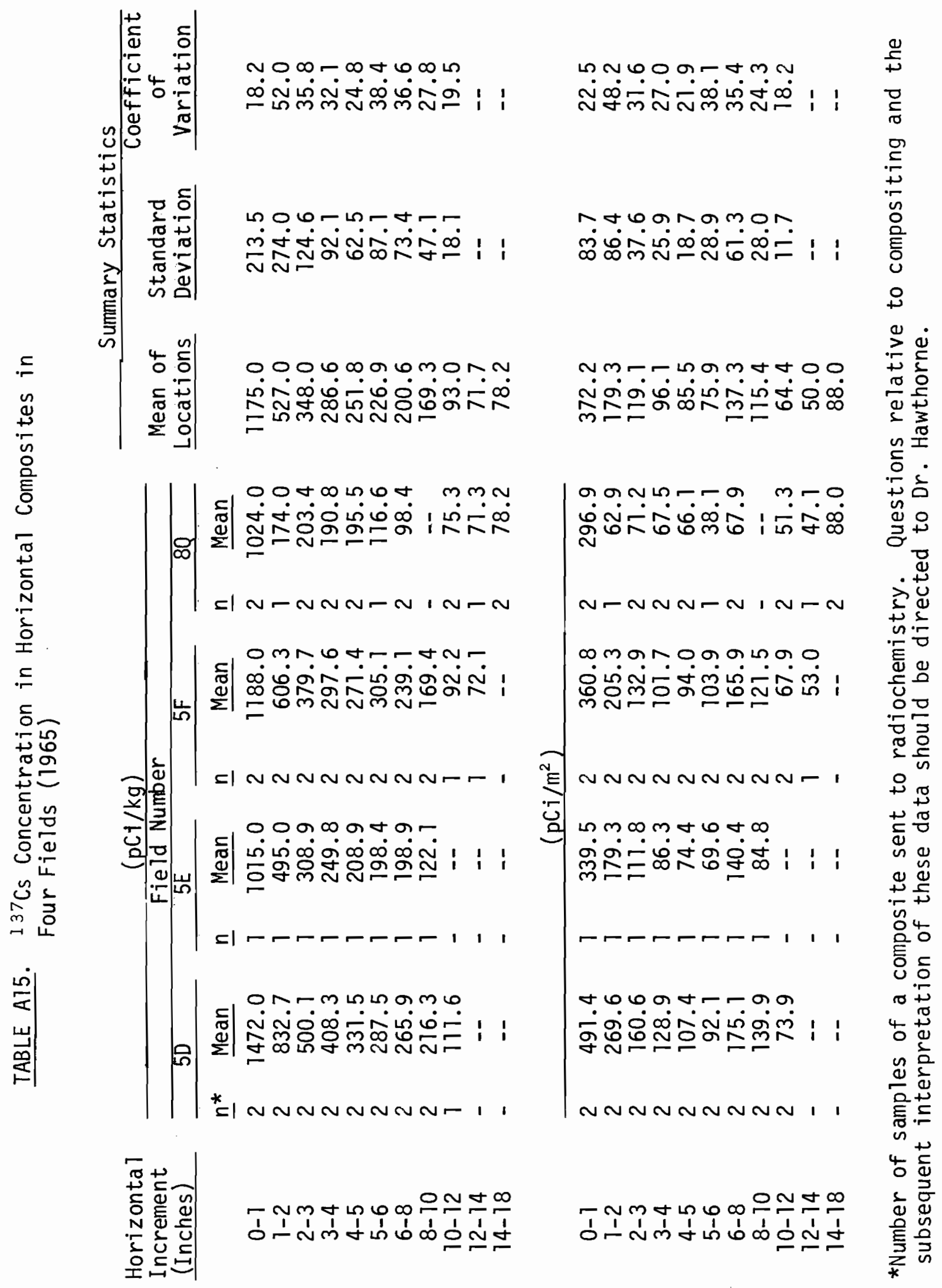




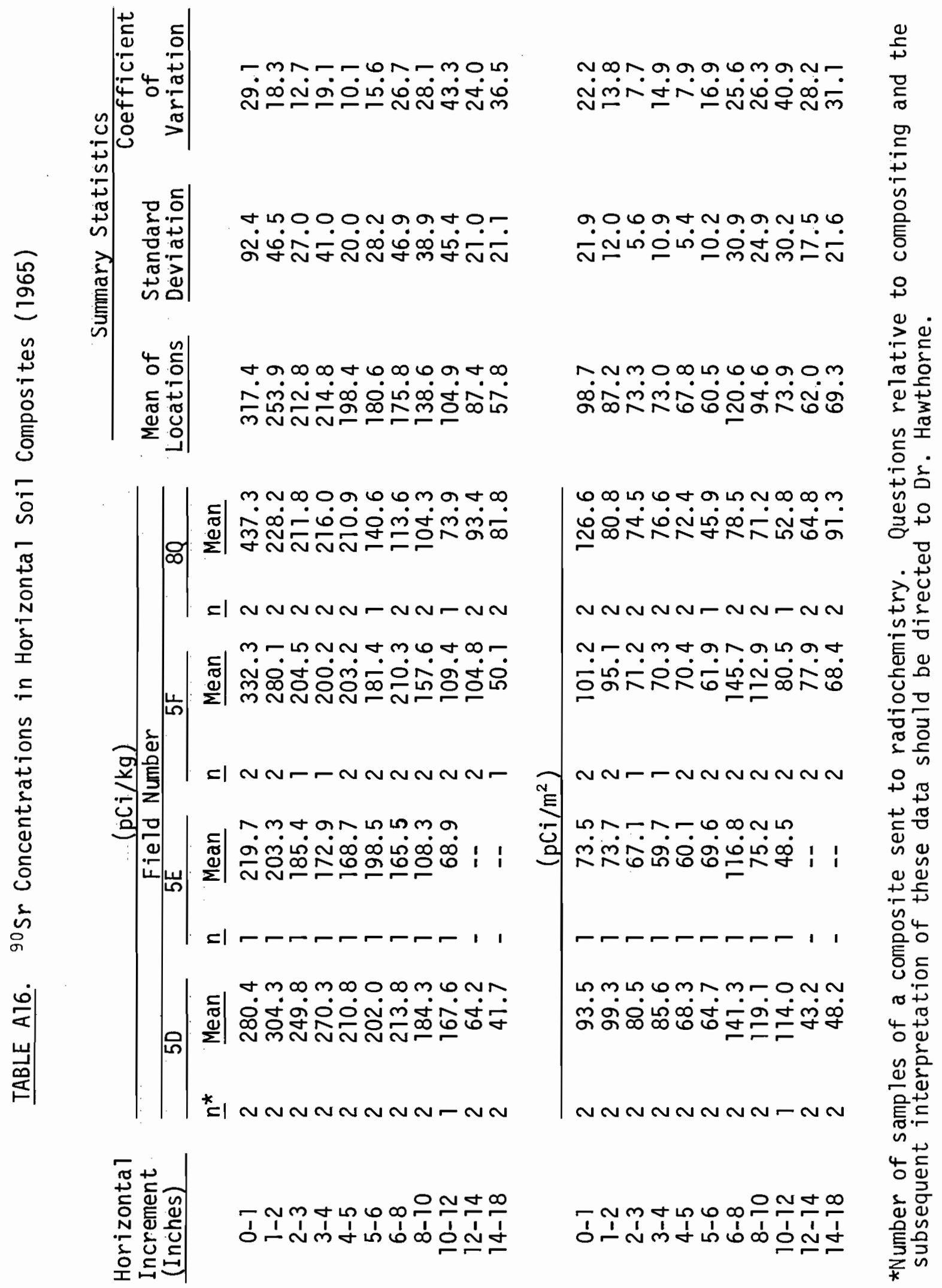




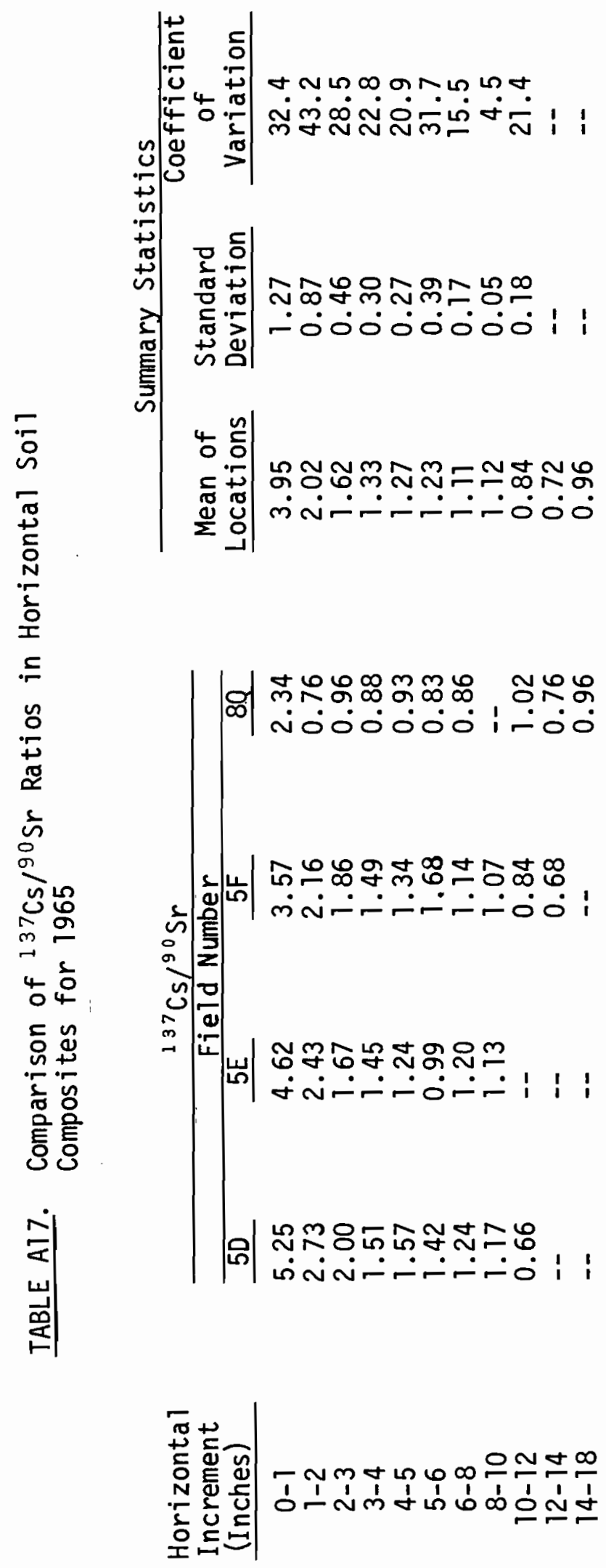




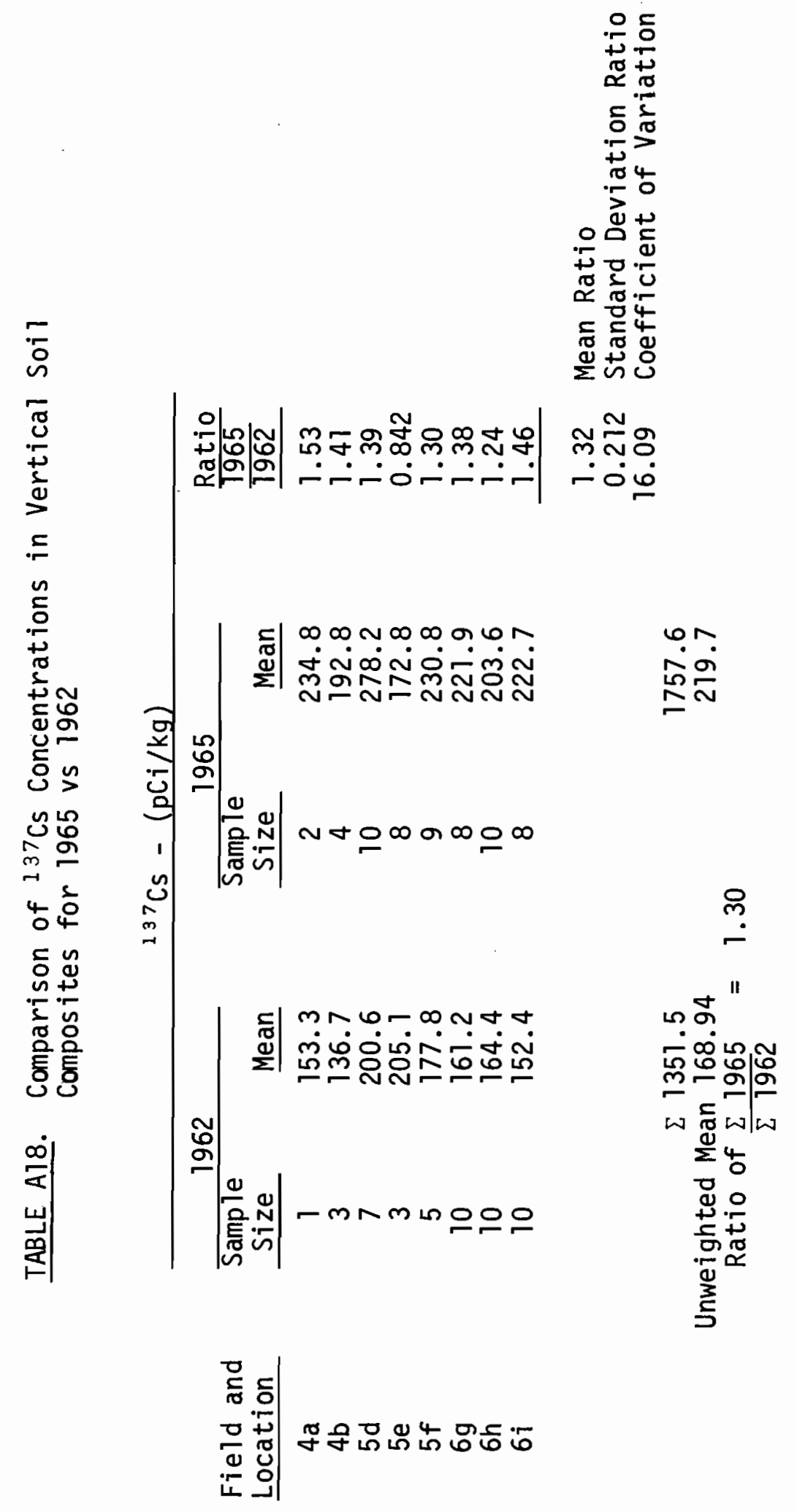




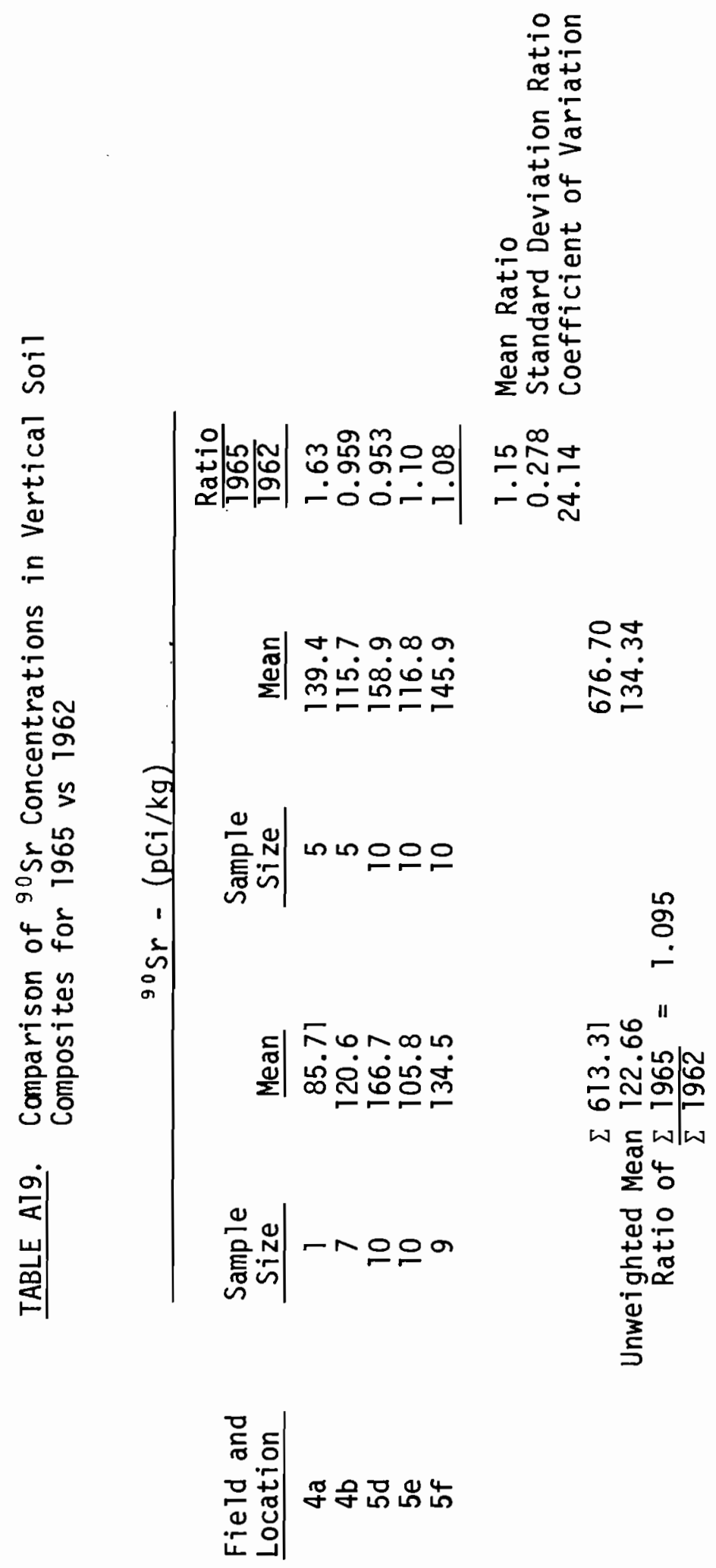



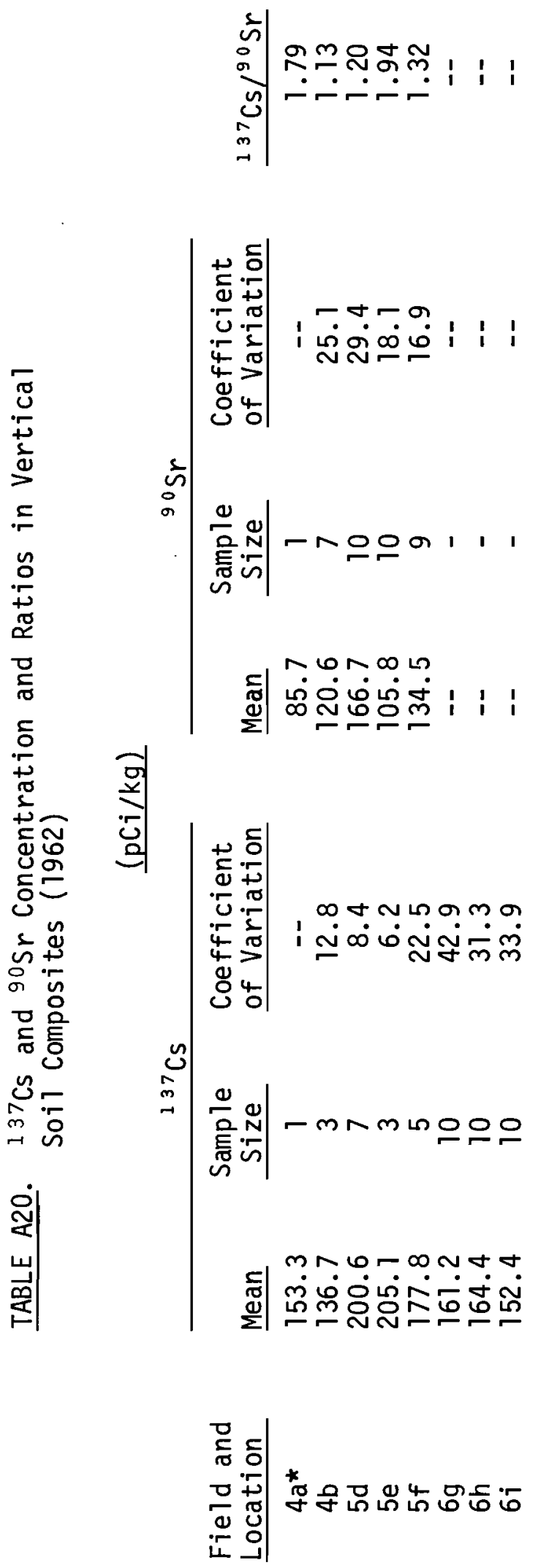

ตำตับำ : 1 i

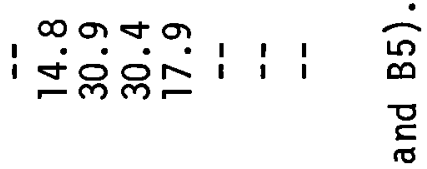

ฮ

ע

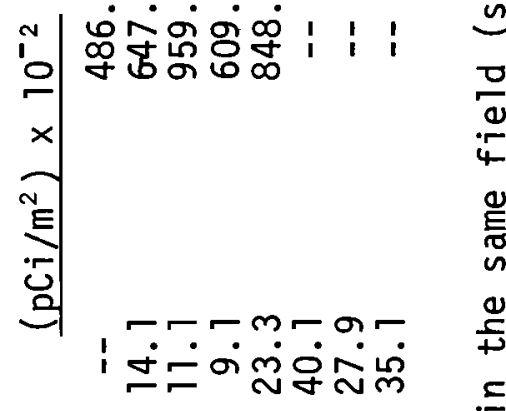

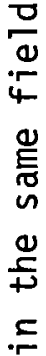

气

-mNmLO응

anooO+wo

iீம்

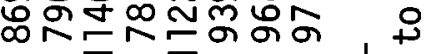

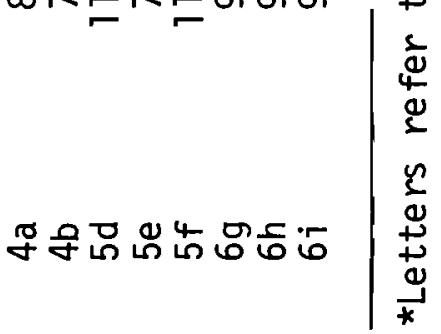




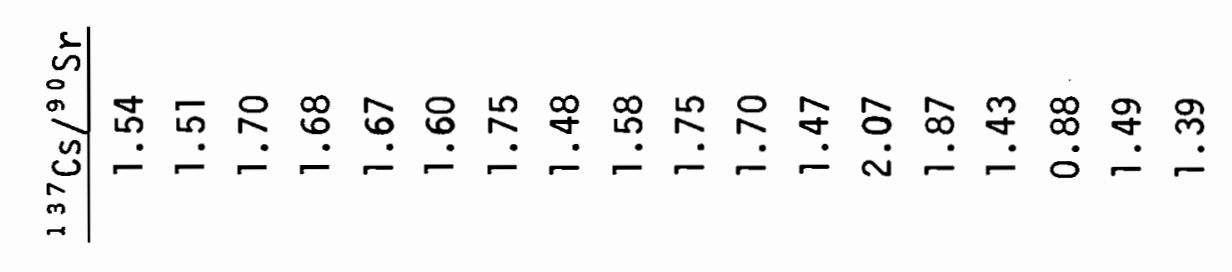

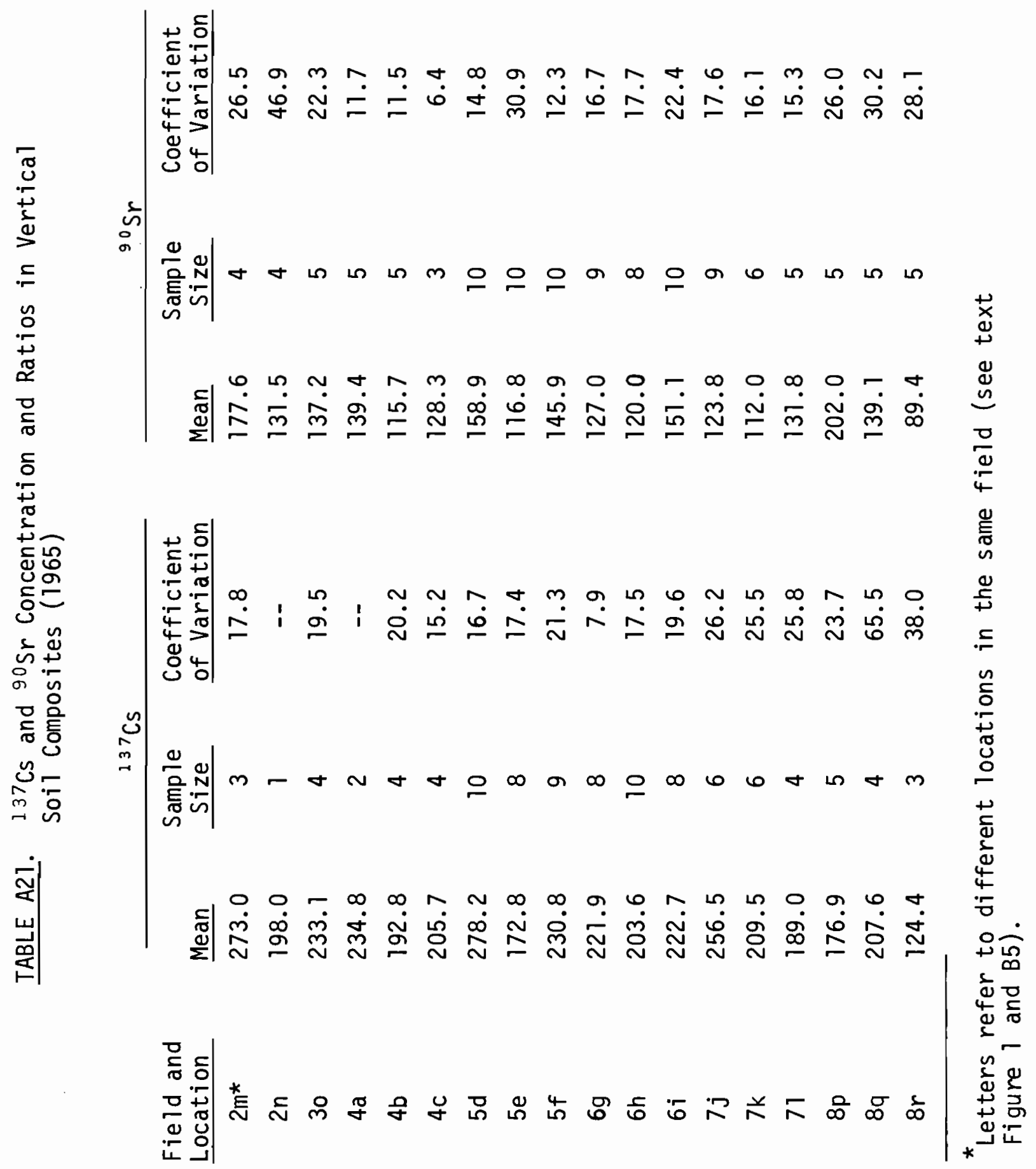




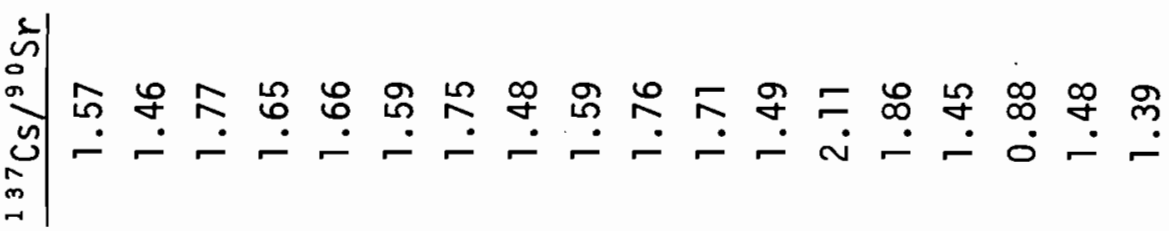

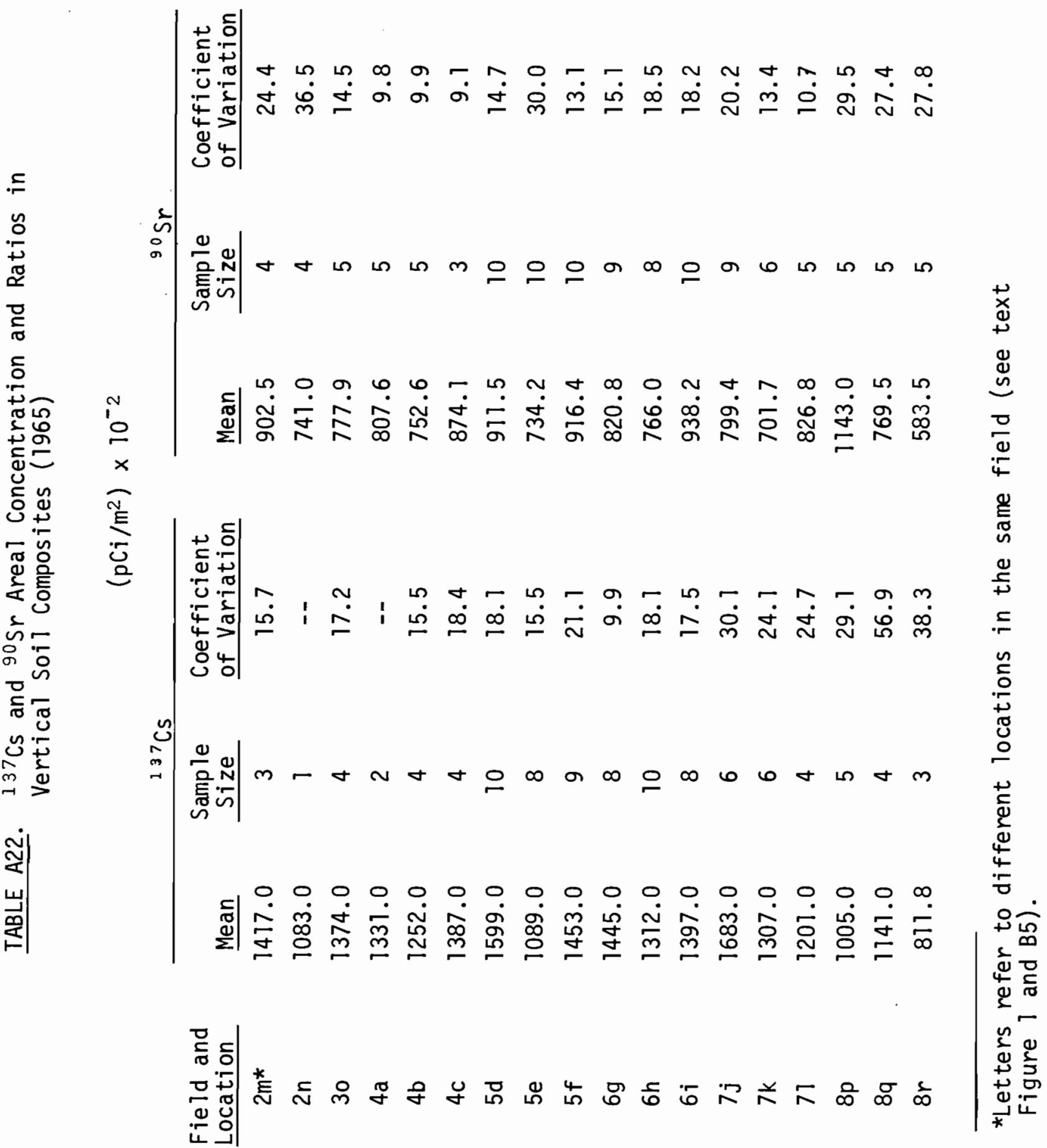




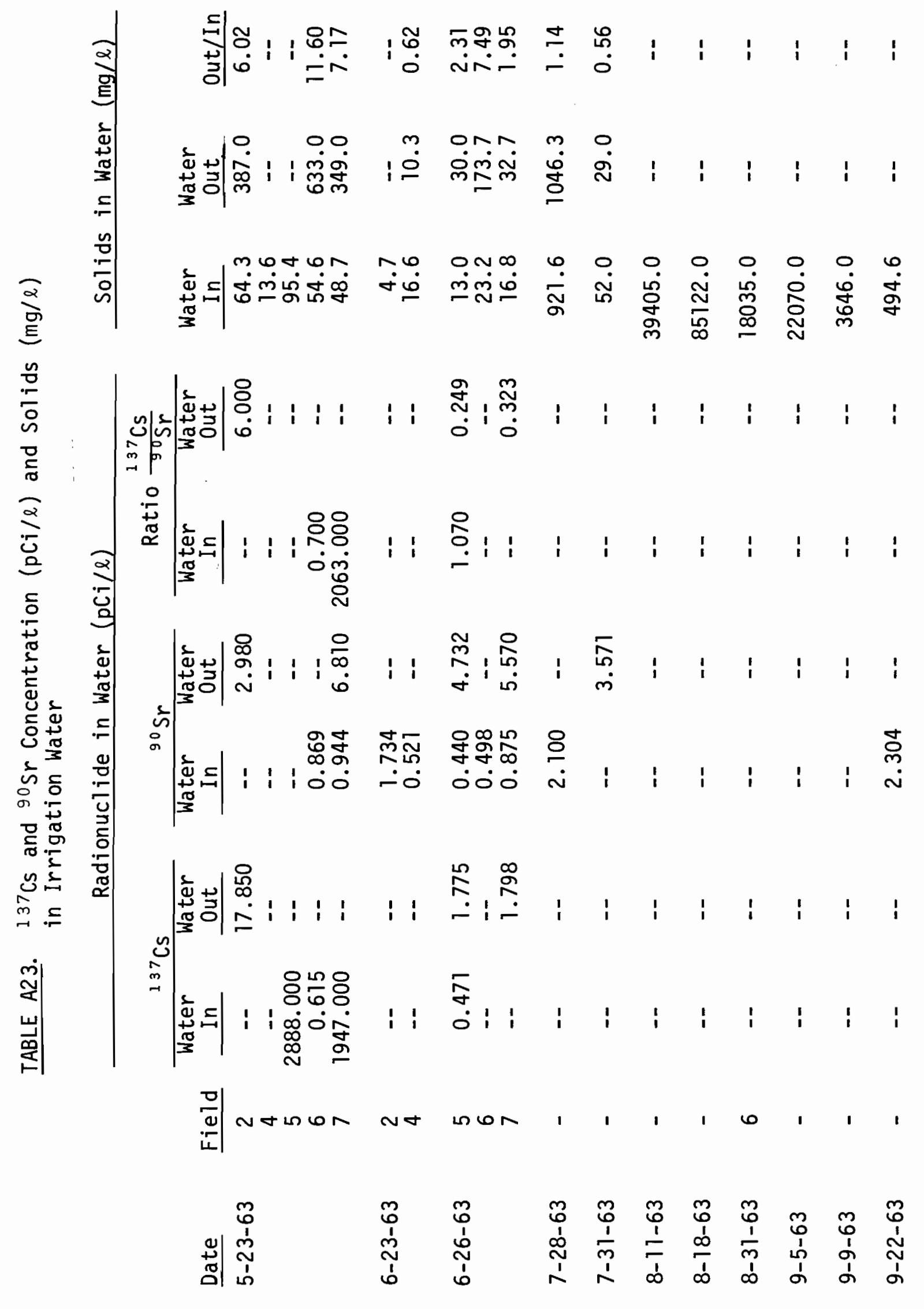




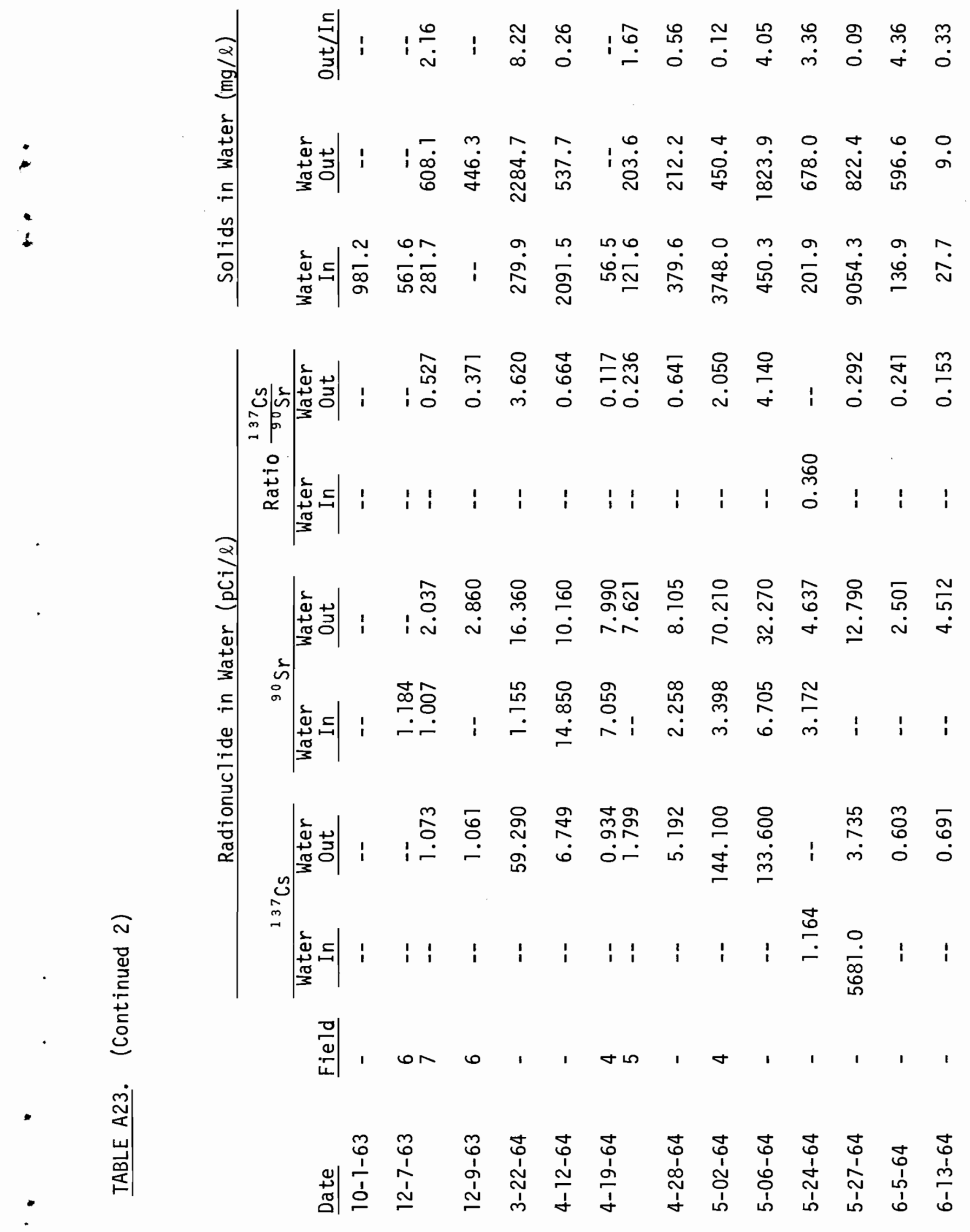




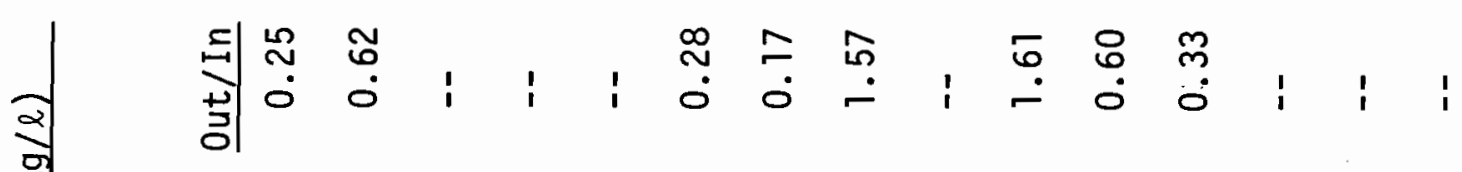

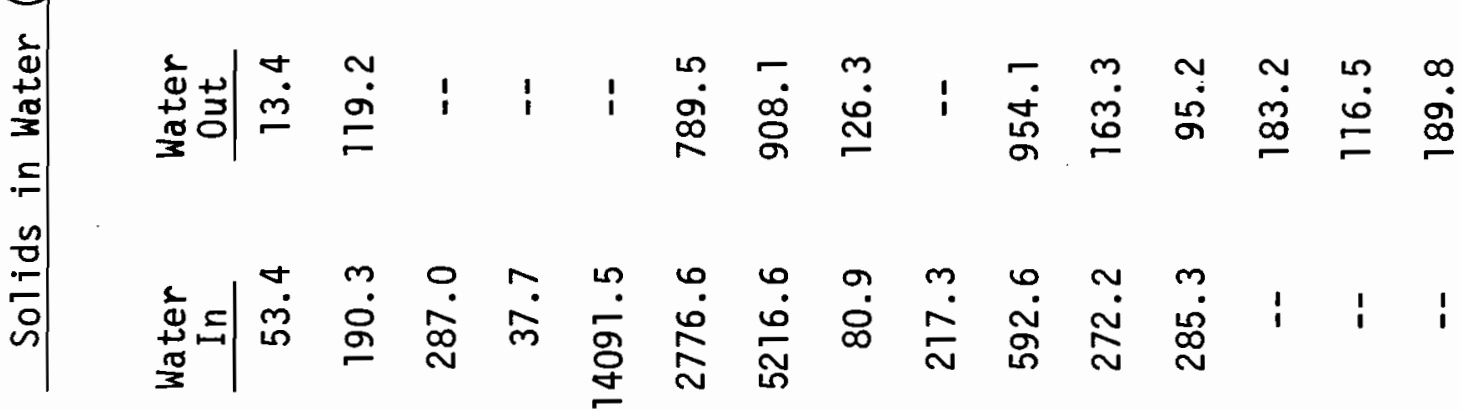

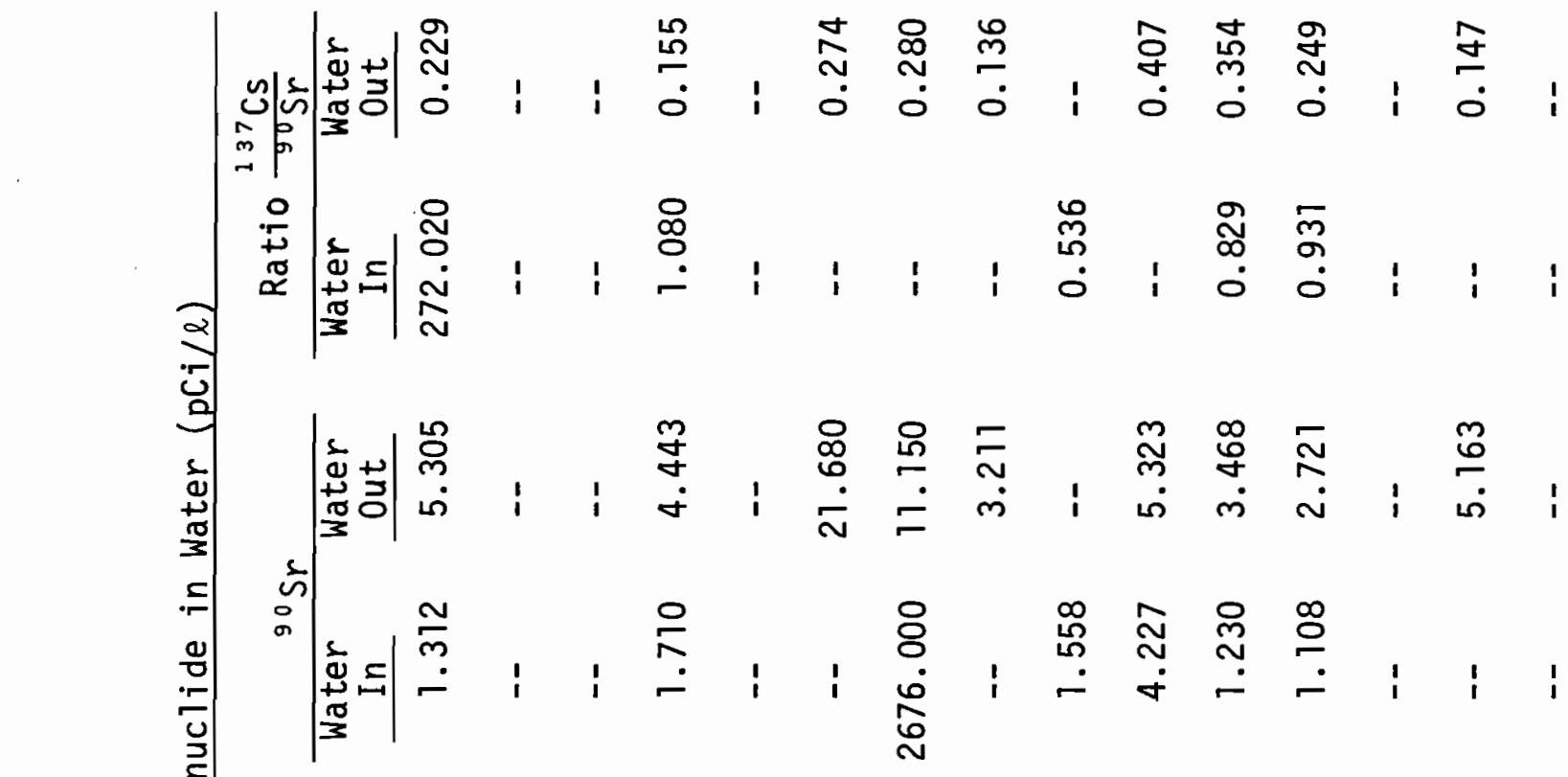

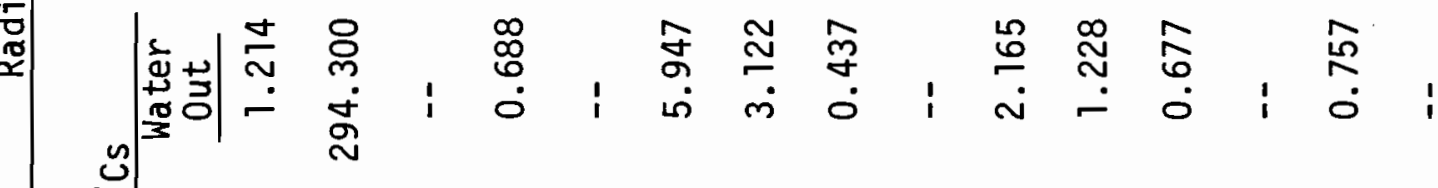

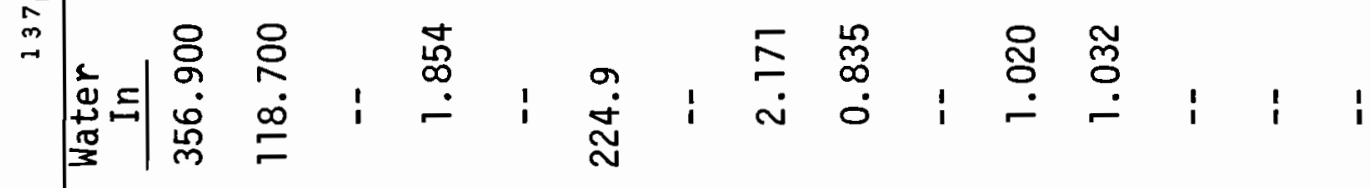
$\begin{array}{llllllllllll}\frac{0}{0} & \frac{0}{4}\end{array}$

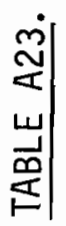

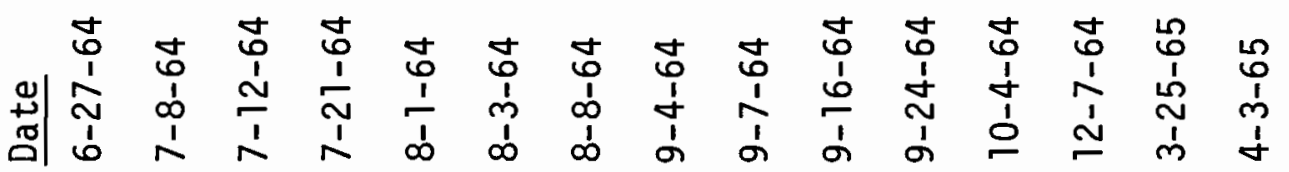




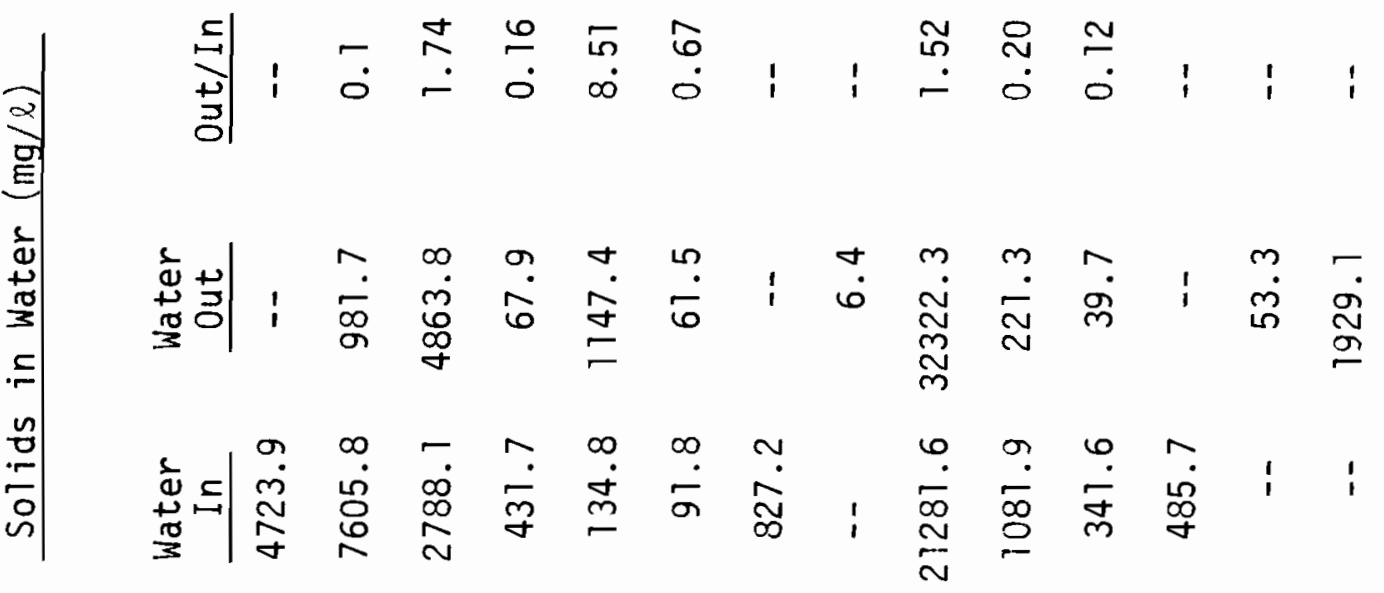

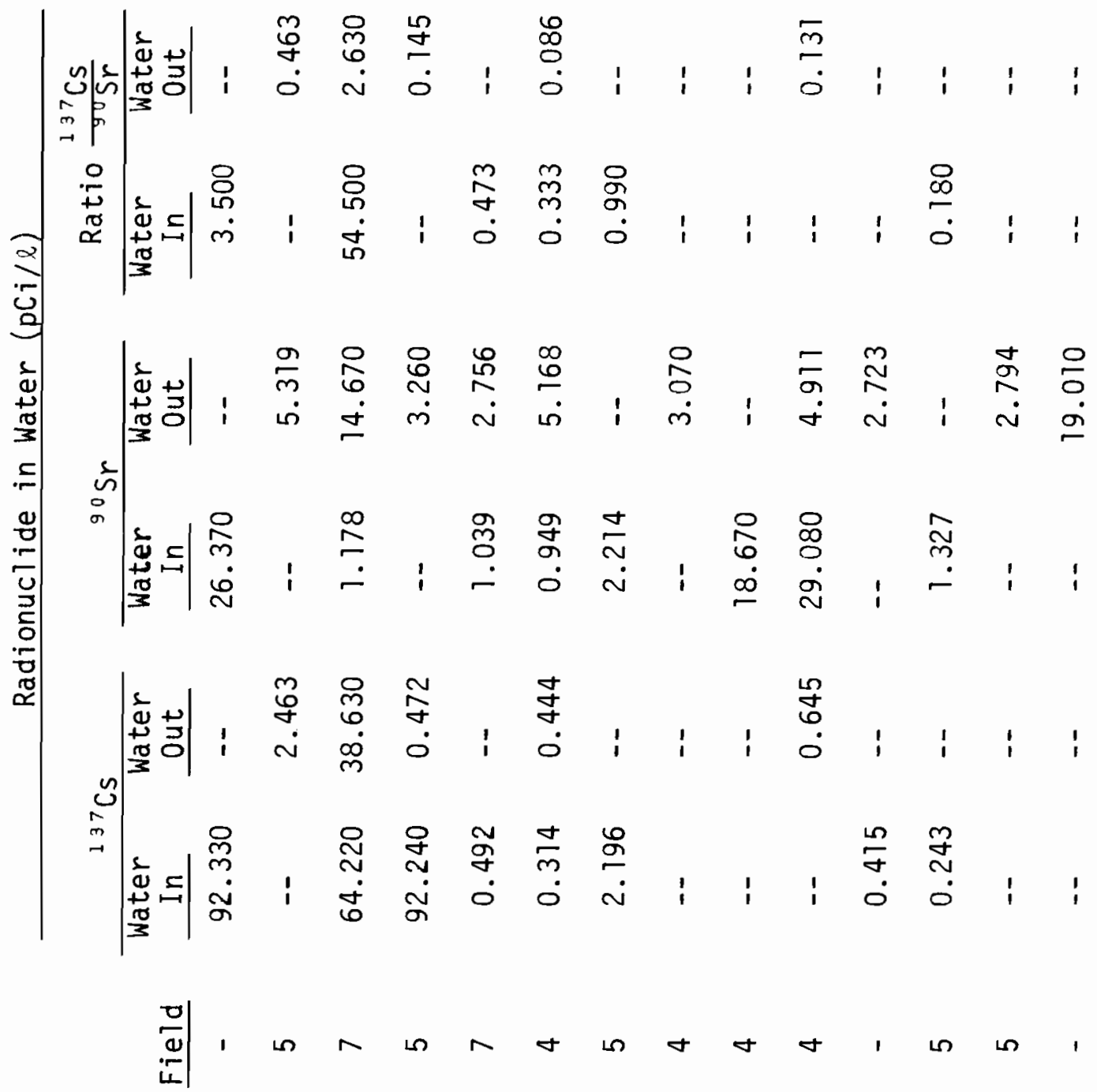




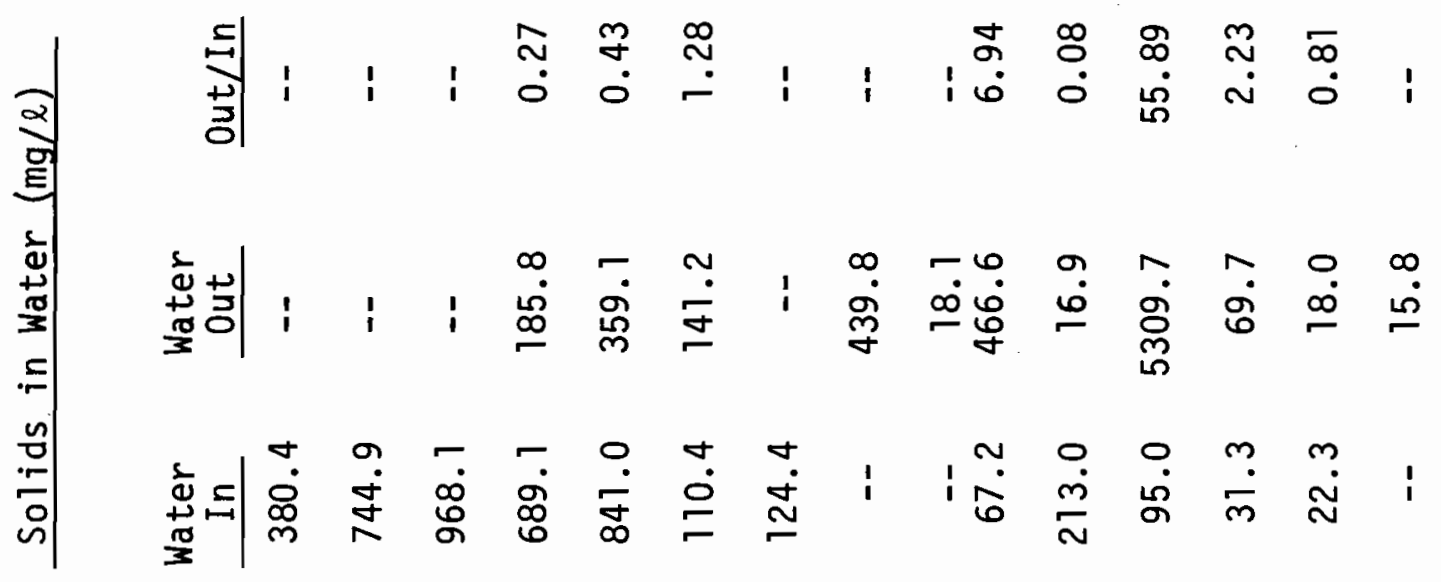

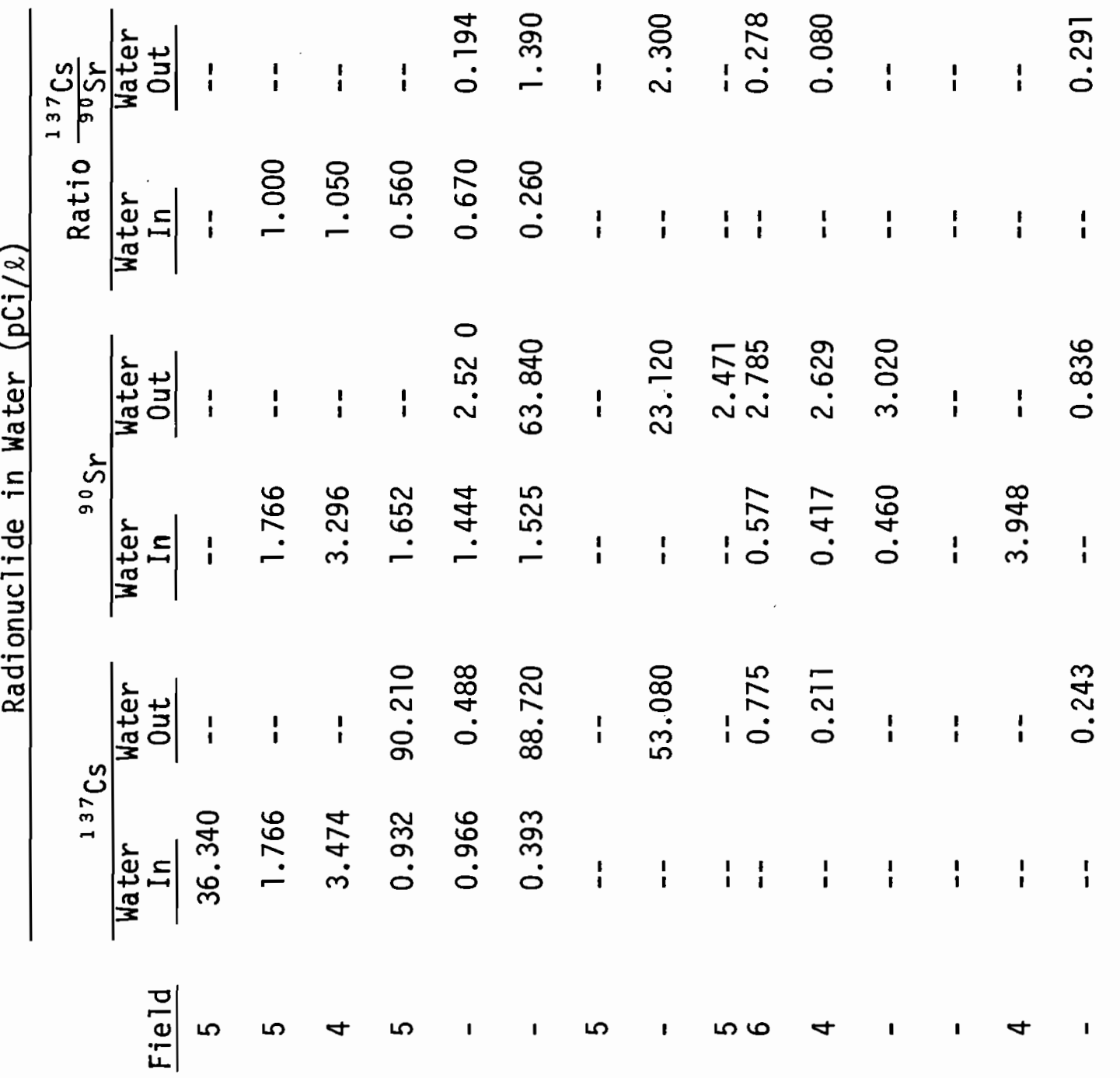

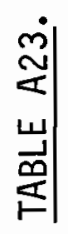

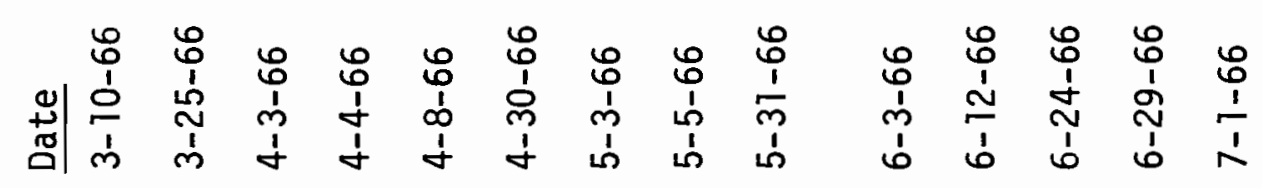




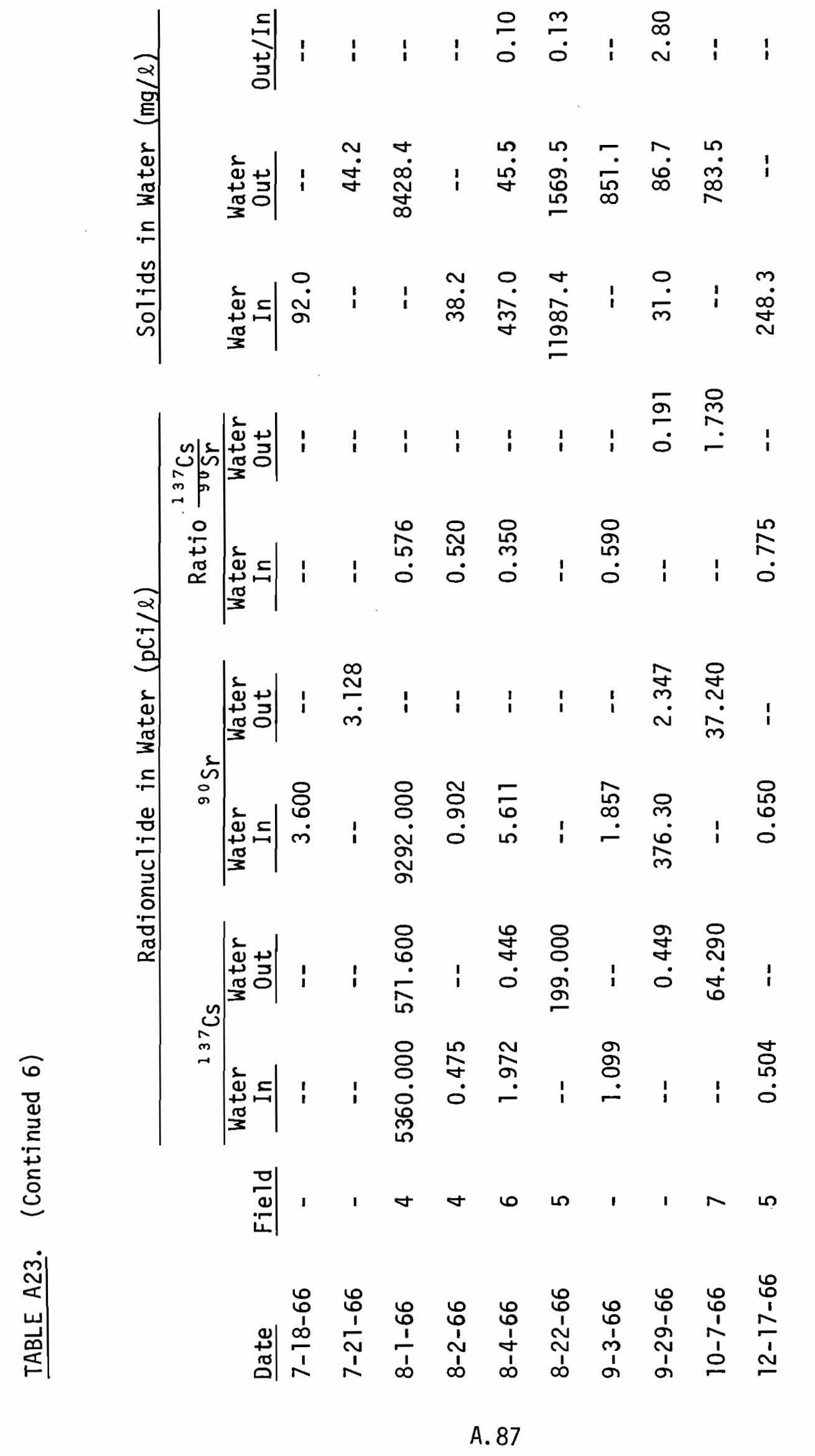




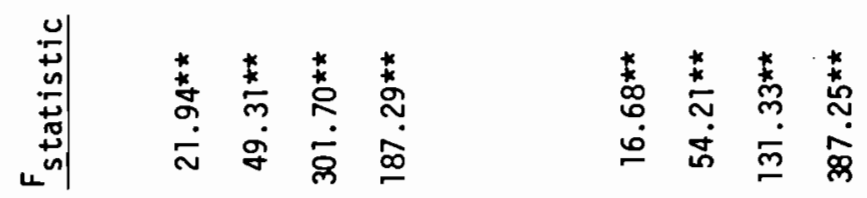
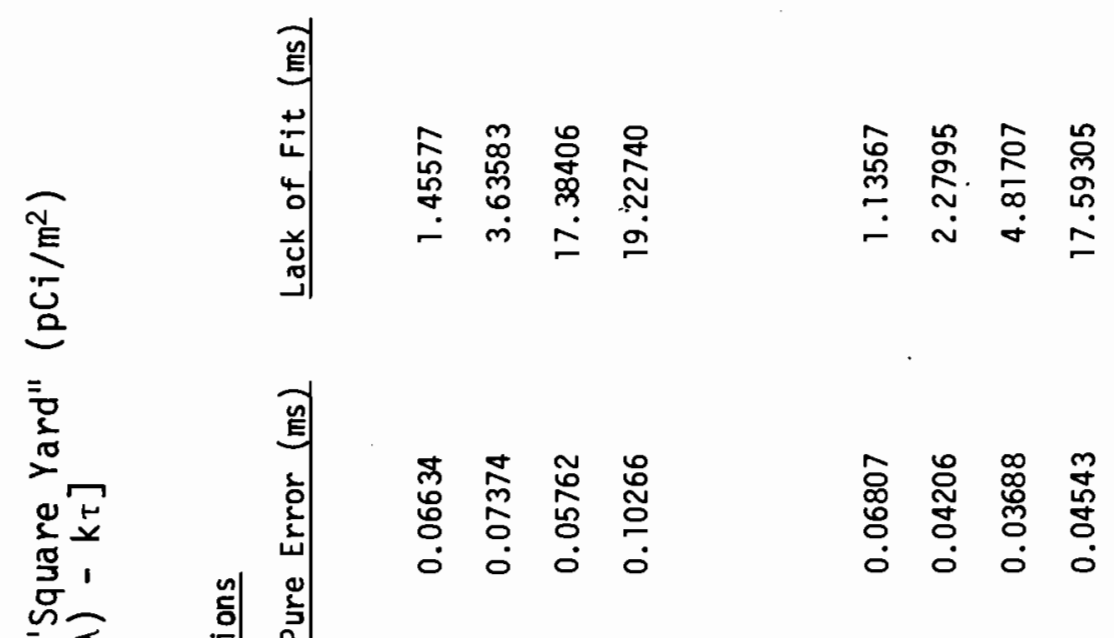

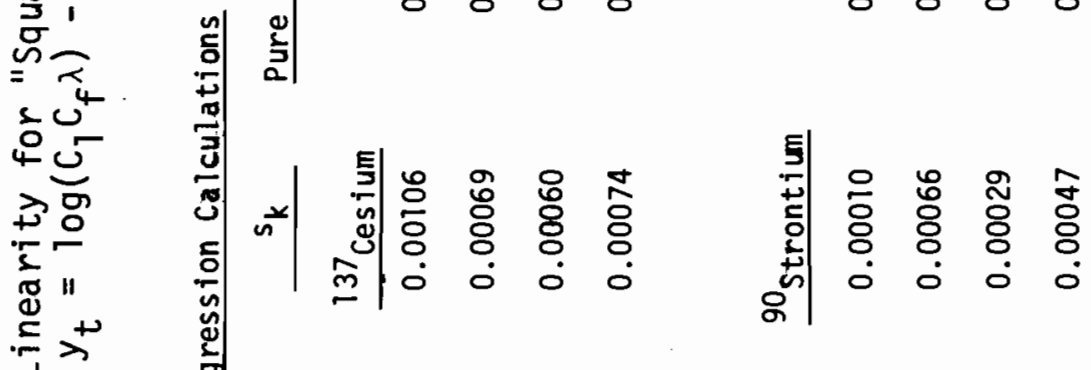

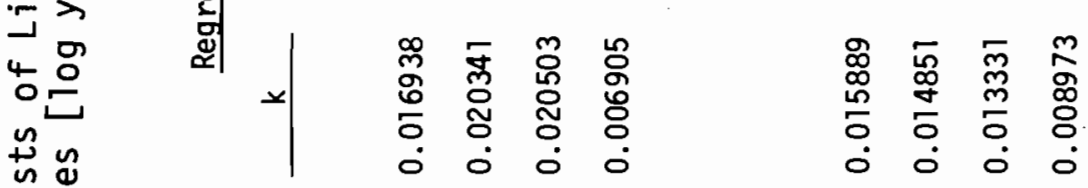

ब.

政高

3

謷

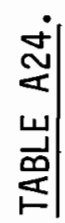

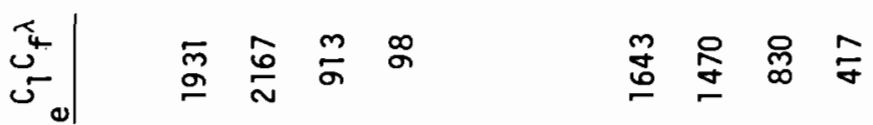

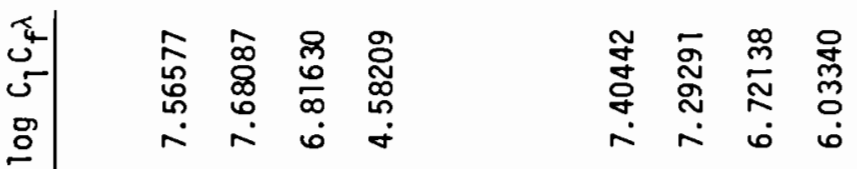

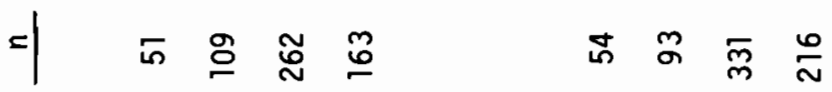

离 


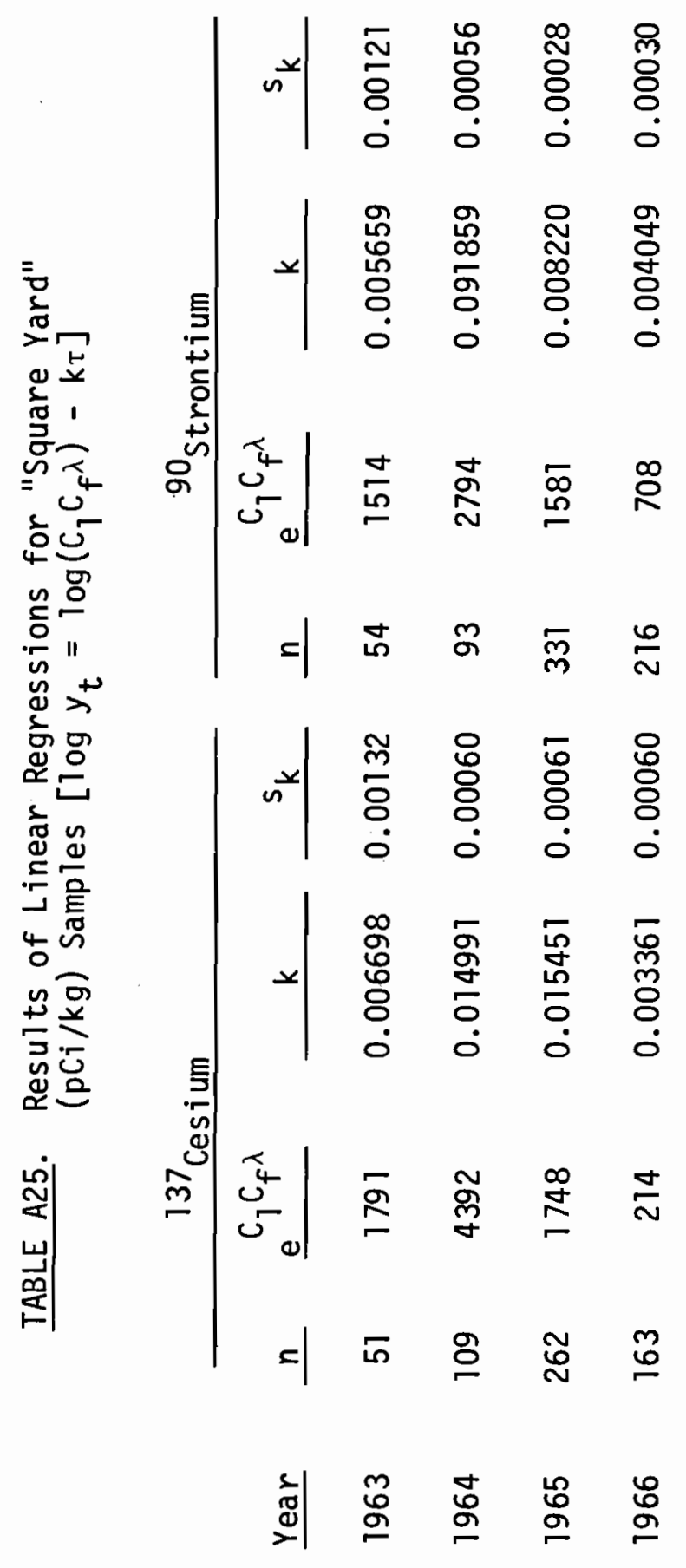




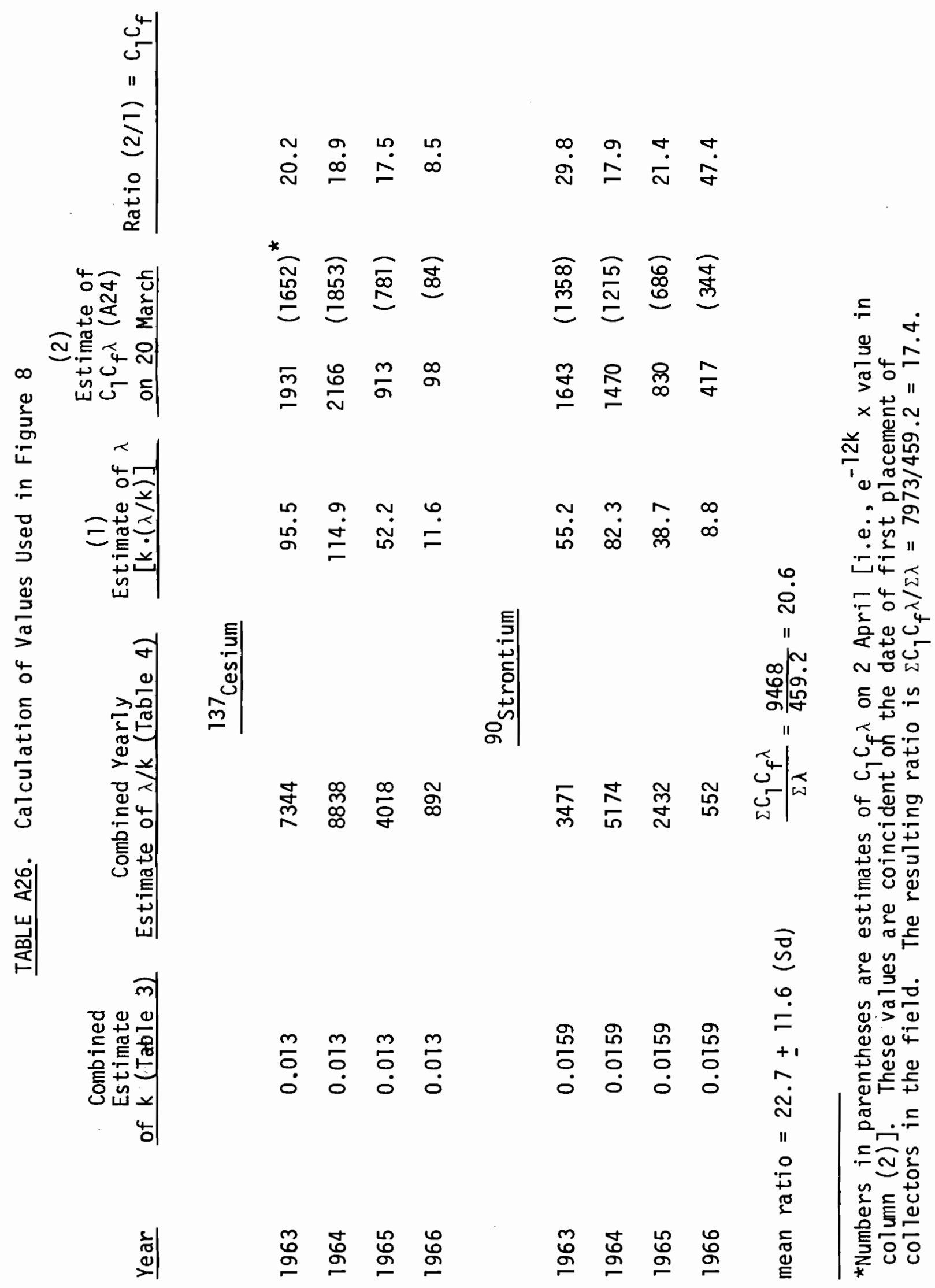


4

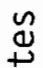

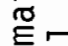

단

놀을

艺胥

¿

$5 \frac{7}{2}$

ब

$\sum$

옫ㄷ

हᄐ ป

인

놑

ᄃ

बे.

豆芒

हิ

s

茖

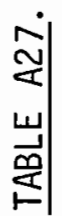

A. 91 

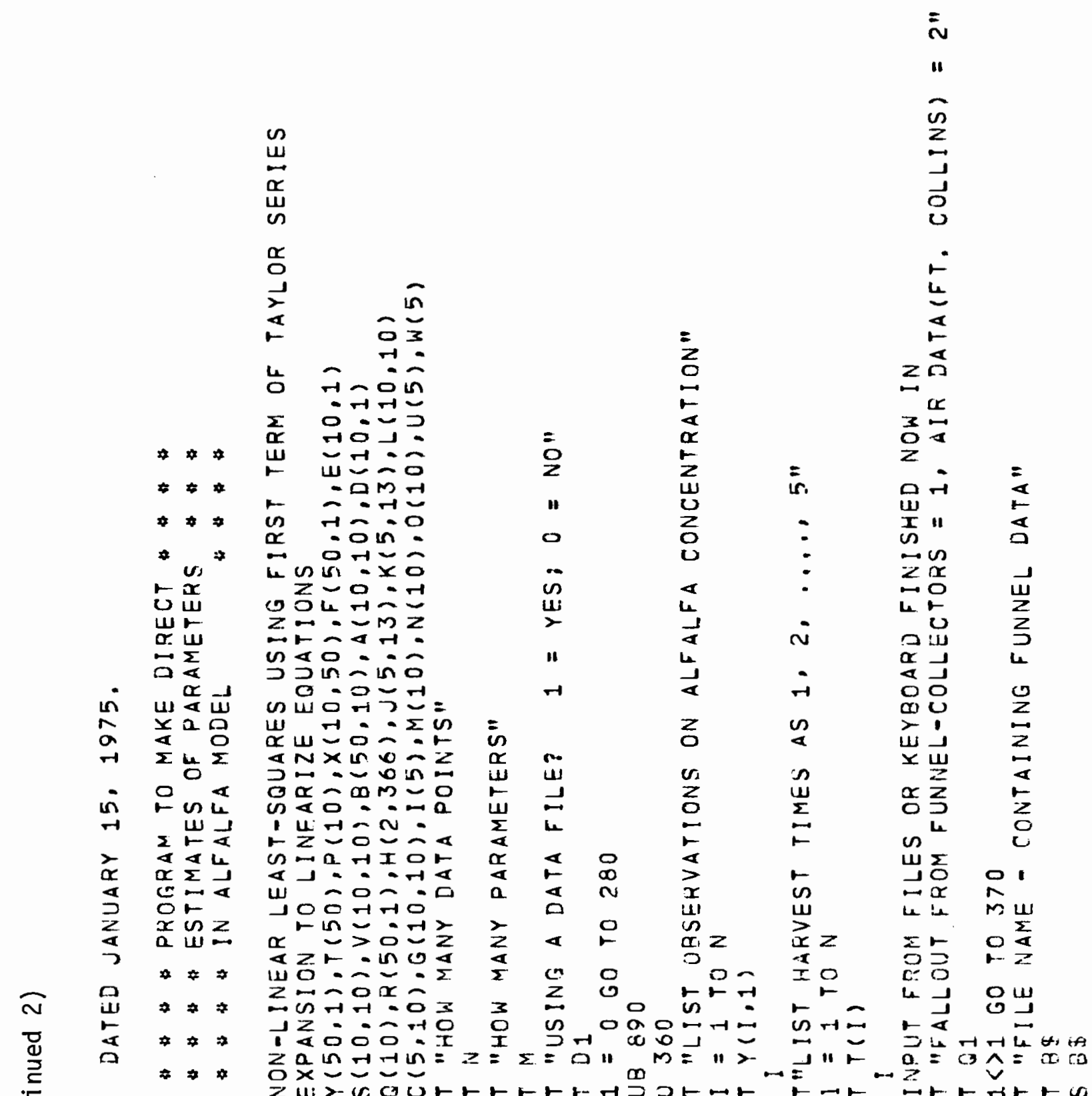

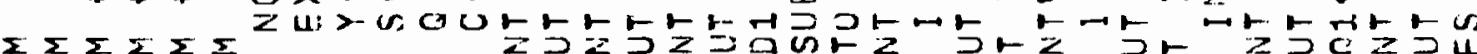

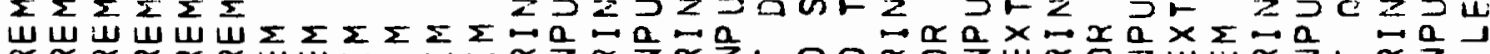

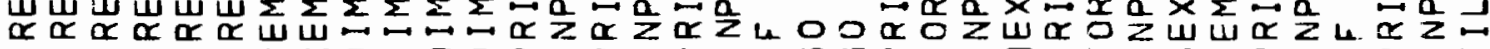

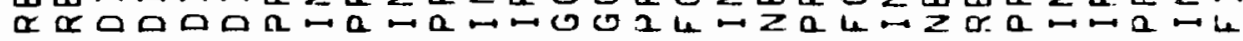

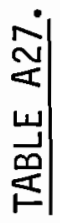

- - - ácó in 


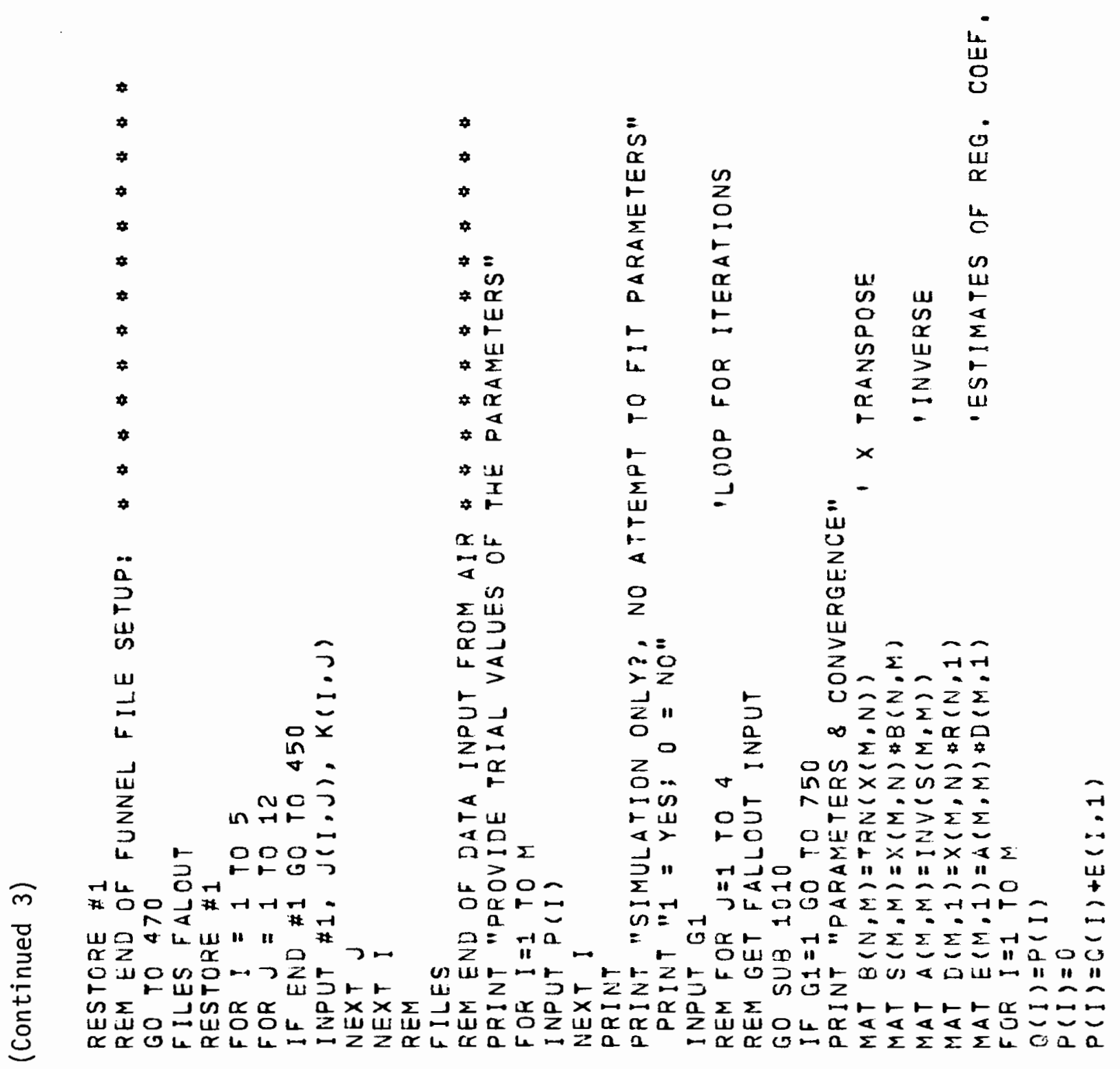

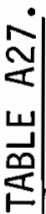

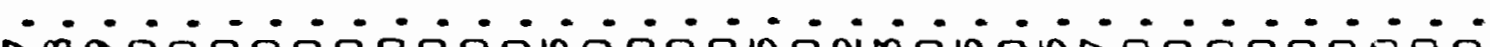
No

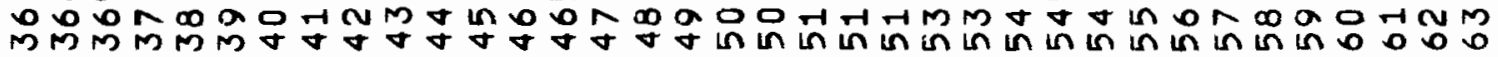




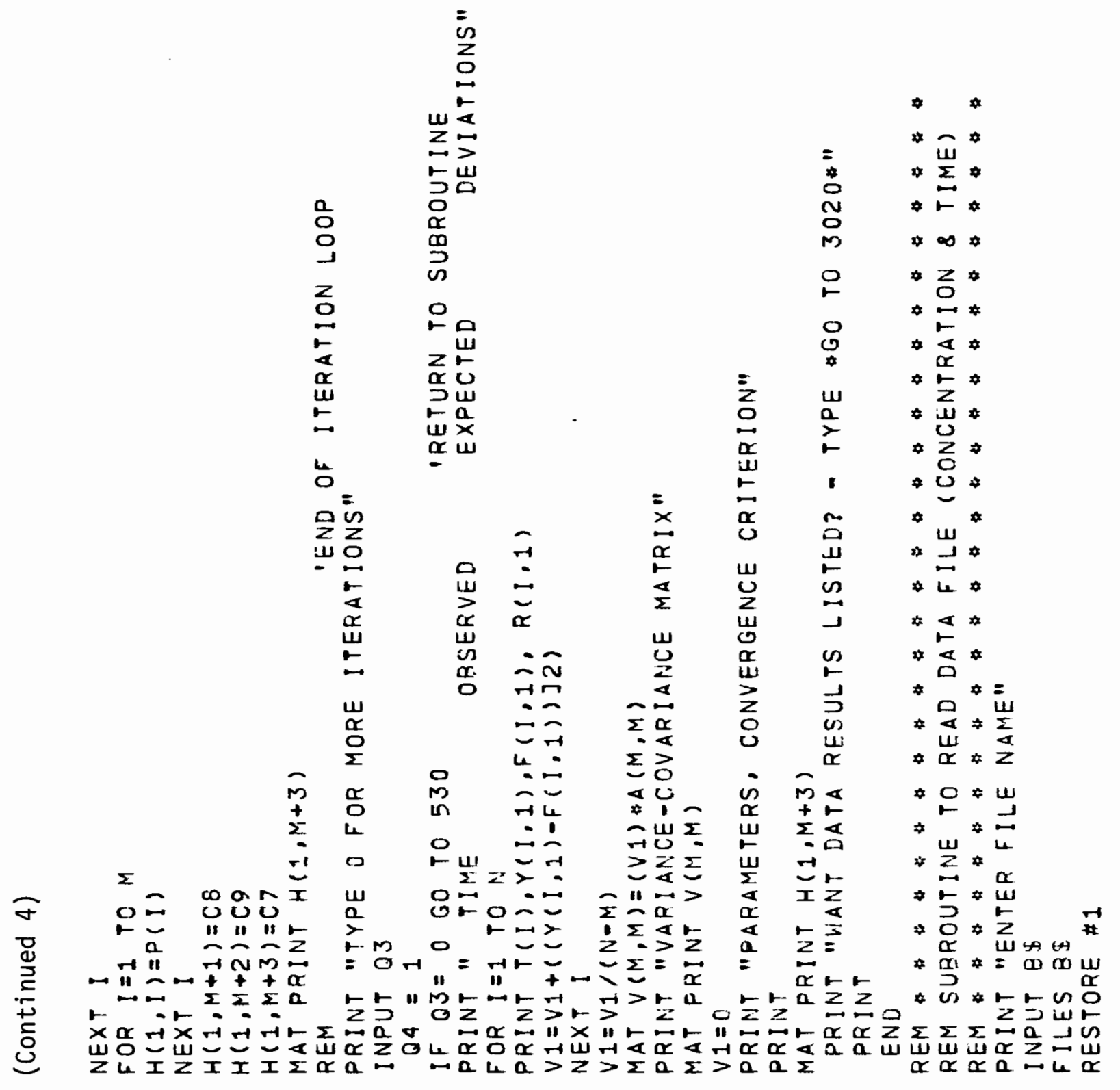

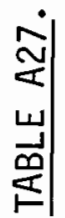

in 


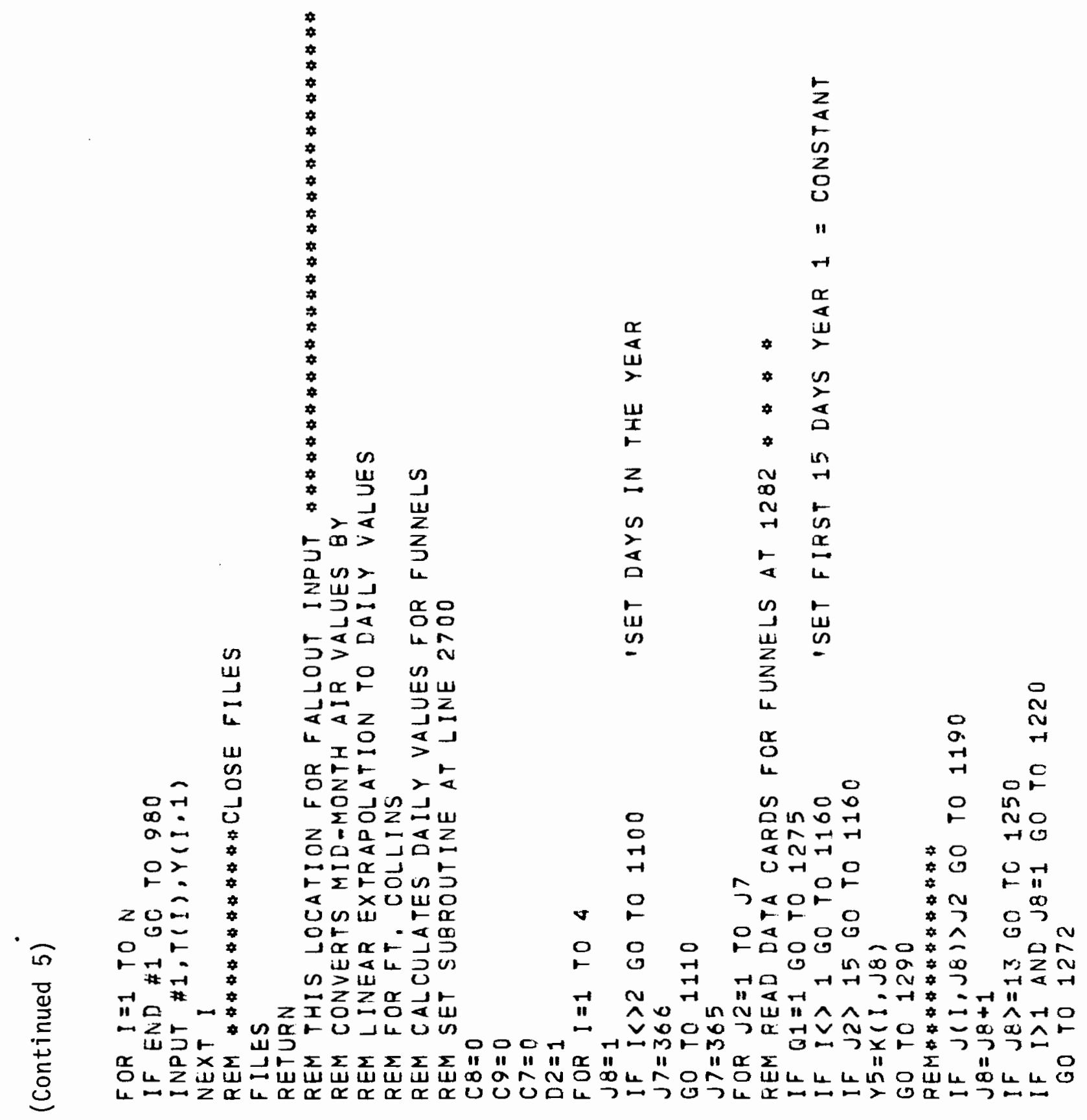

ํㅣㅁ

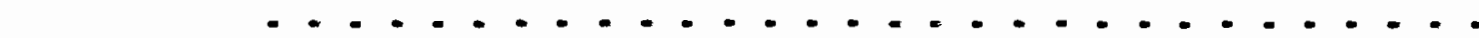
-

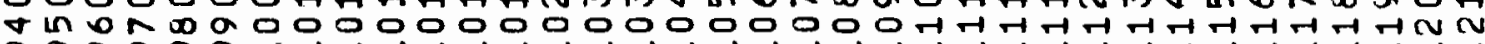
o 0 a o o व 


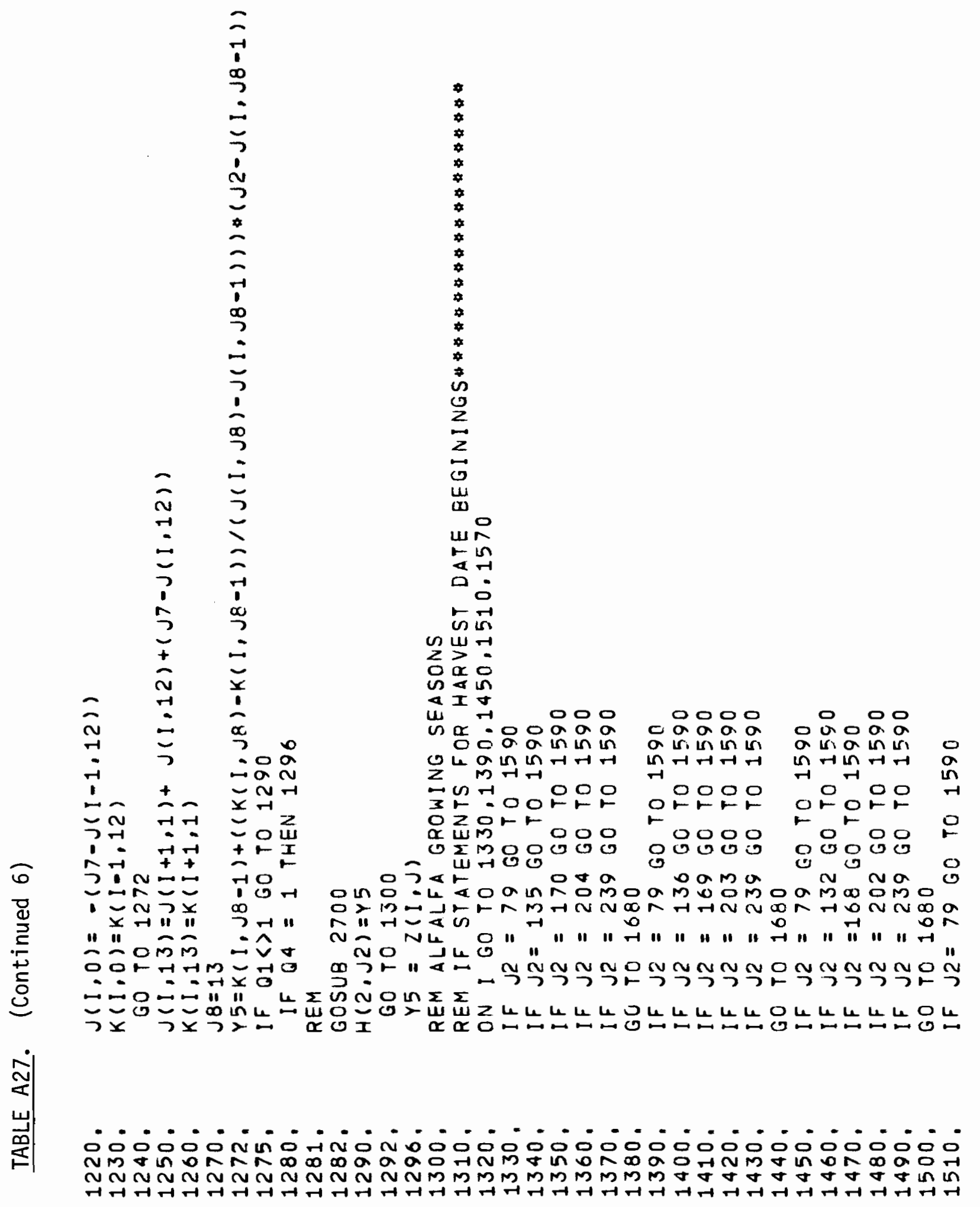




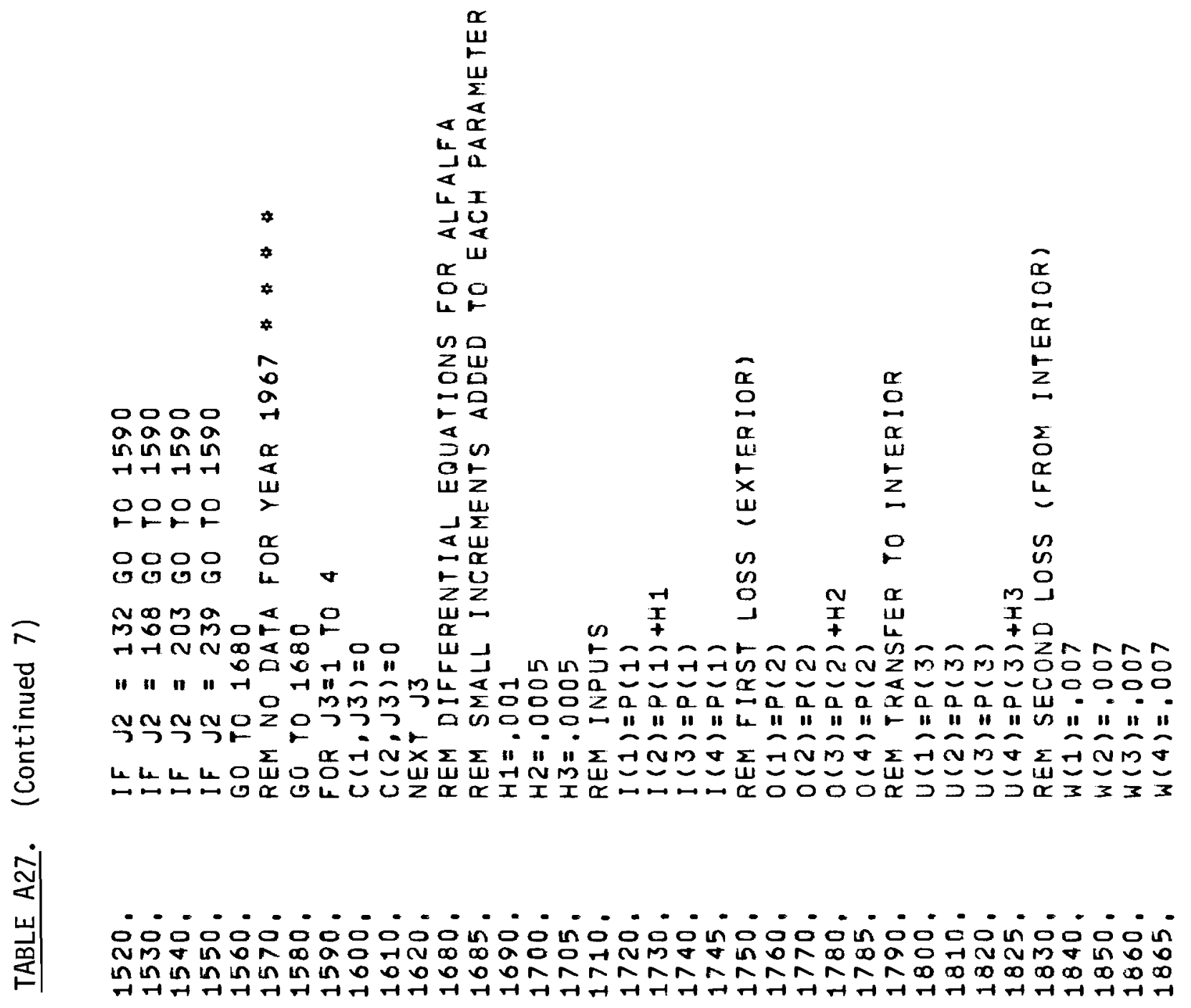




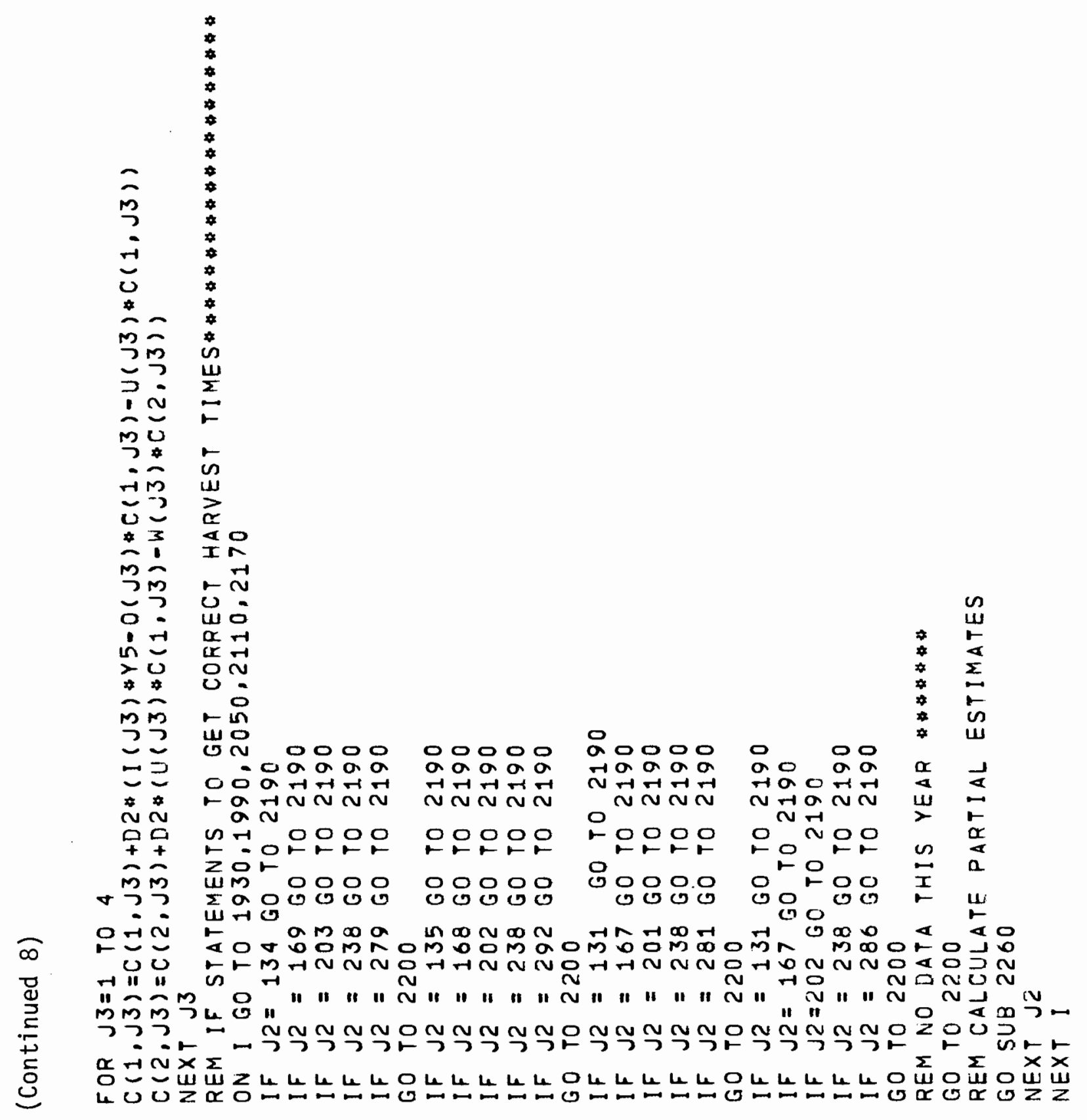

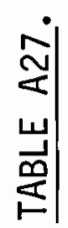

- - - - - - - - - - - - - - - - - - - - - - - - - - - - - - - - -

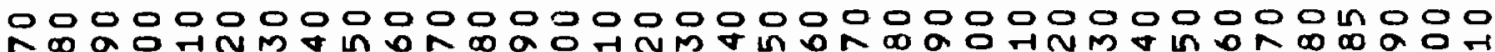

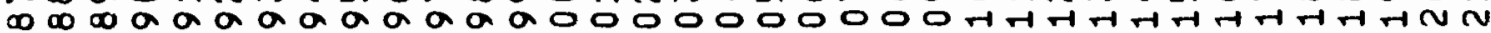
न 


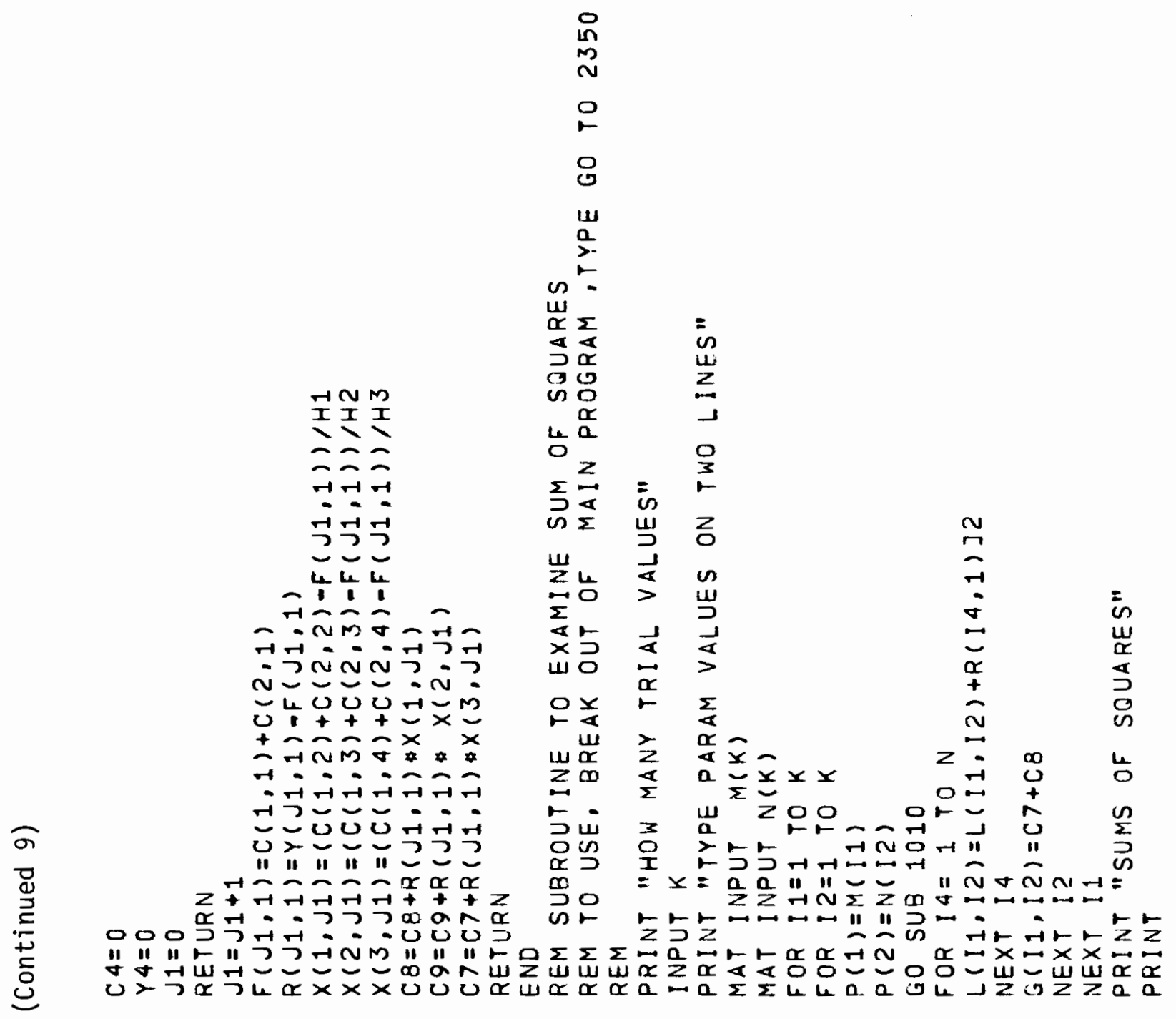

艾

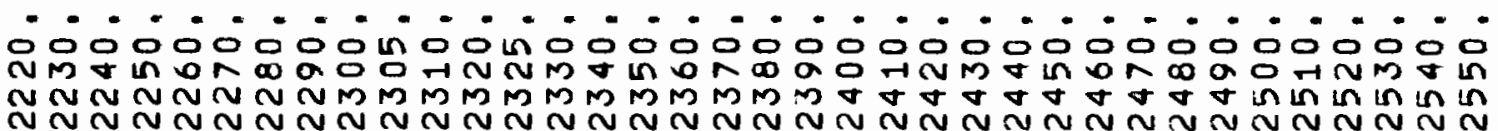




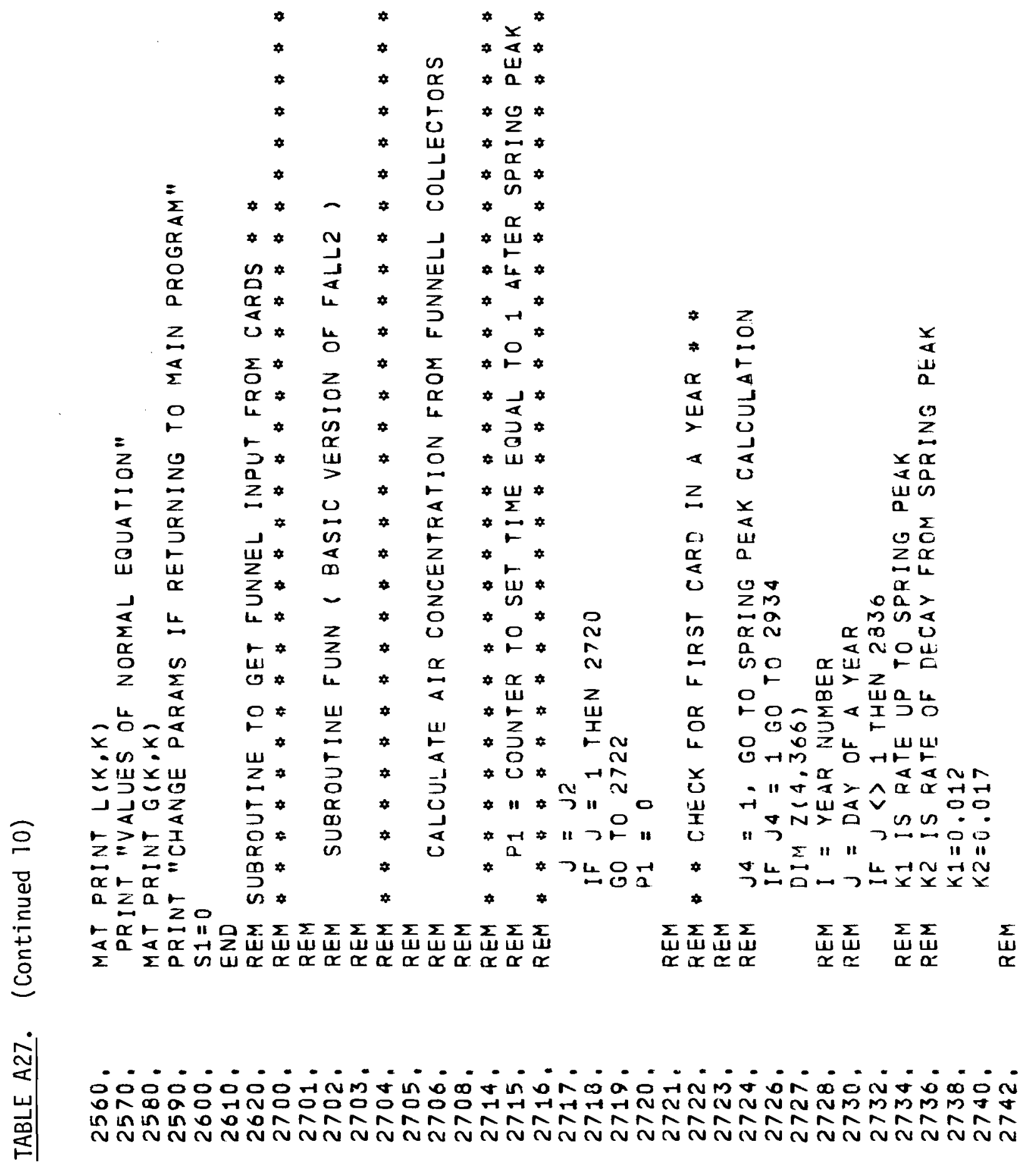




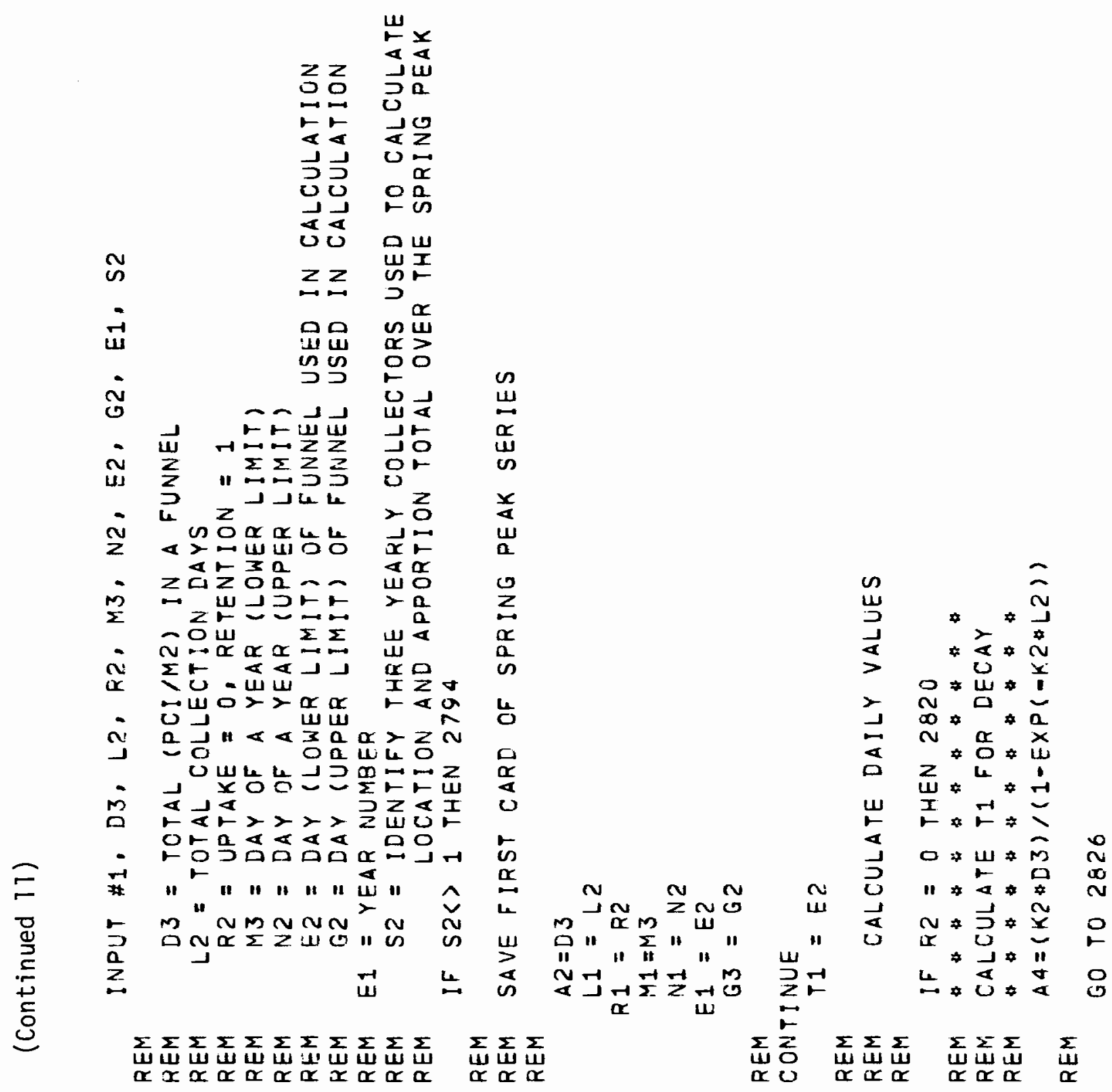

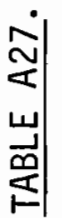

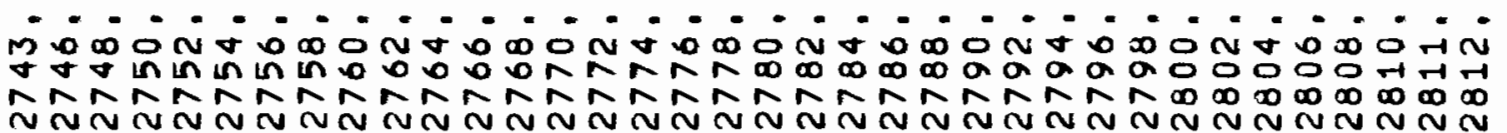




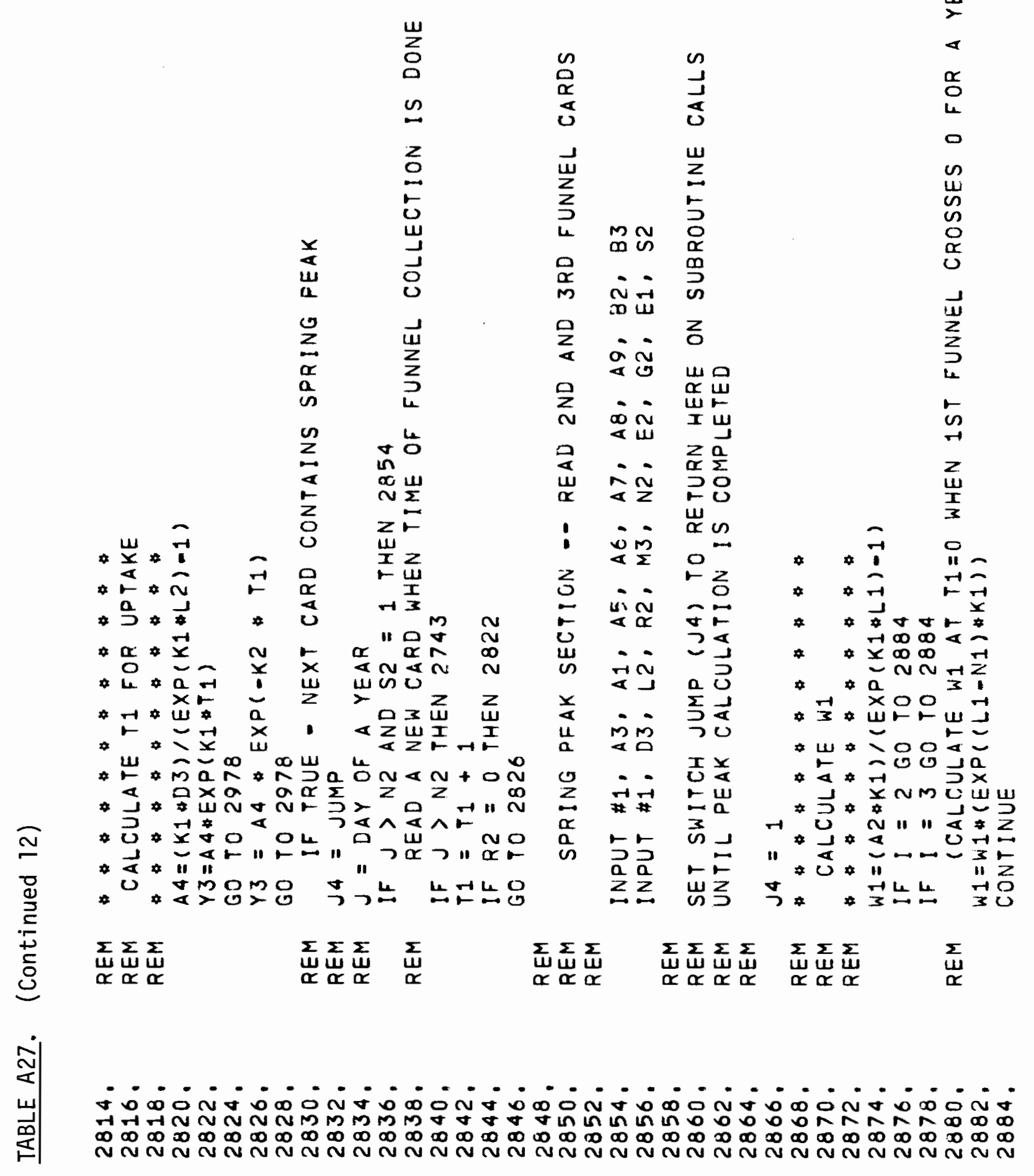




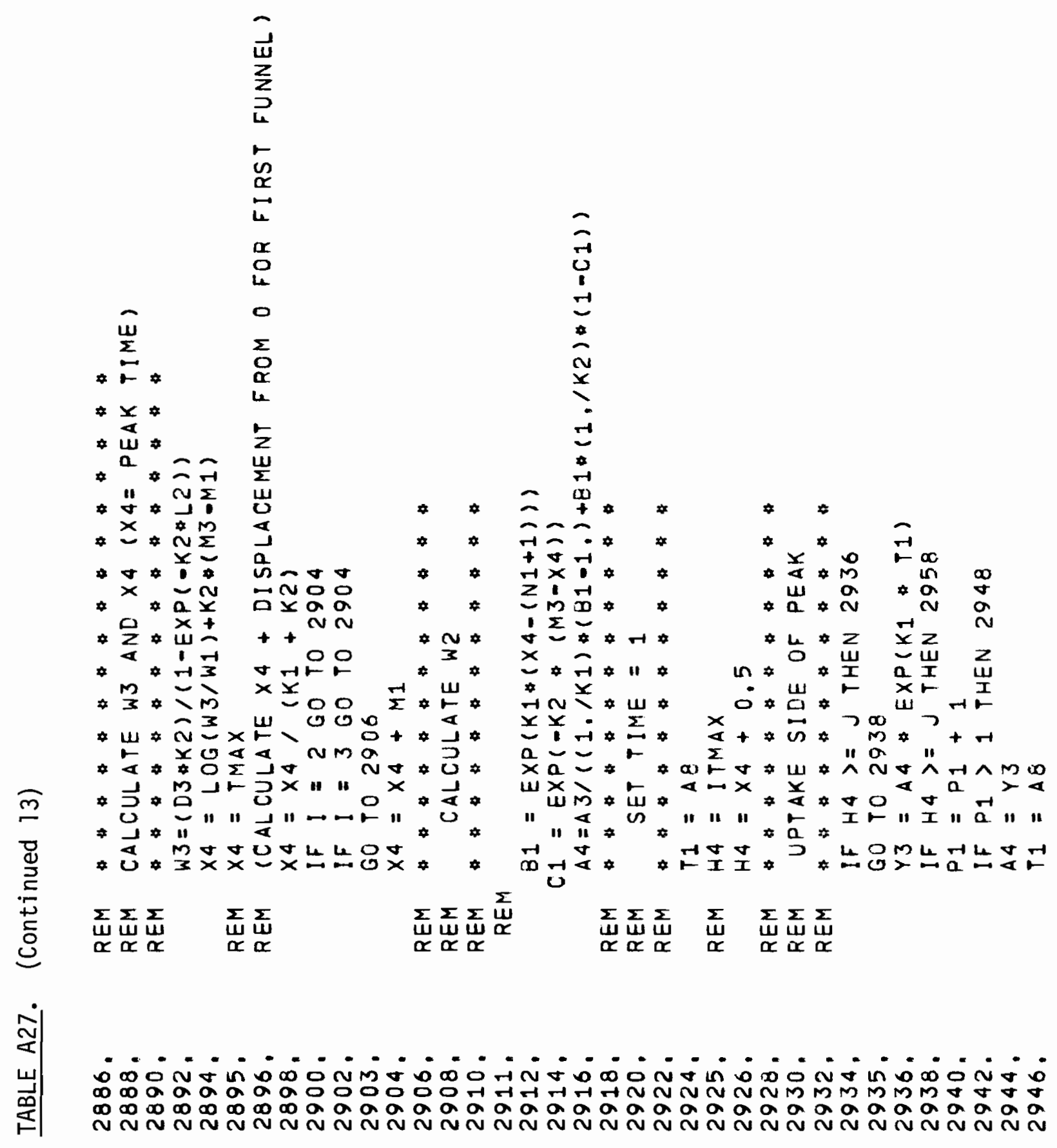




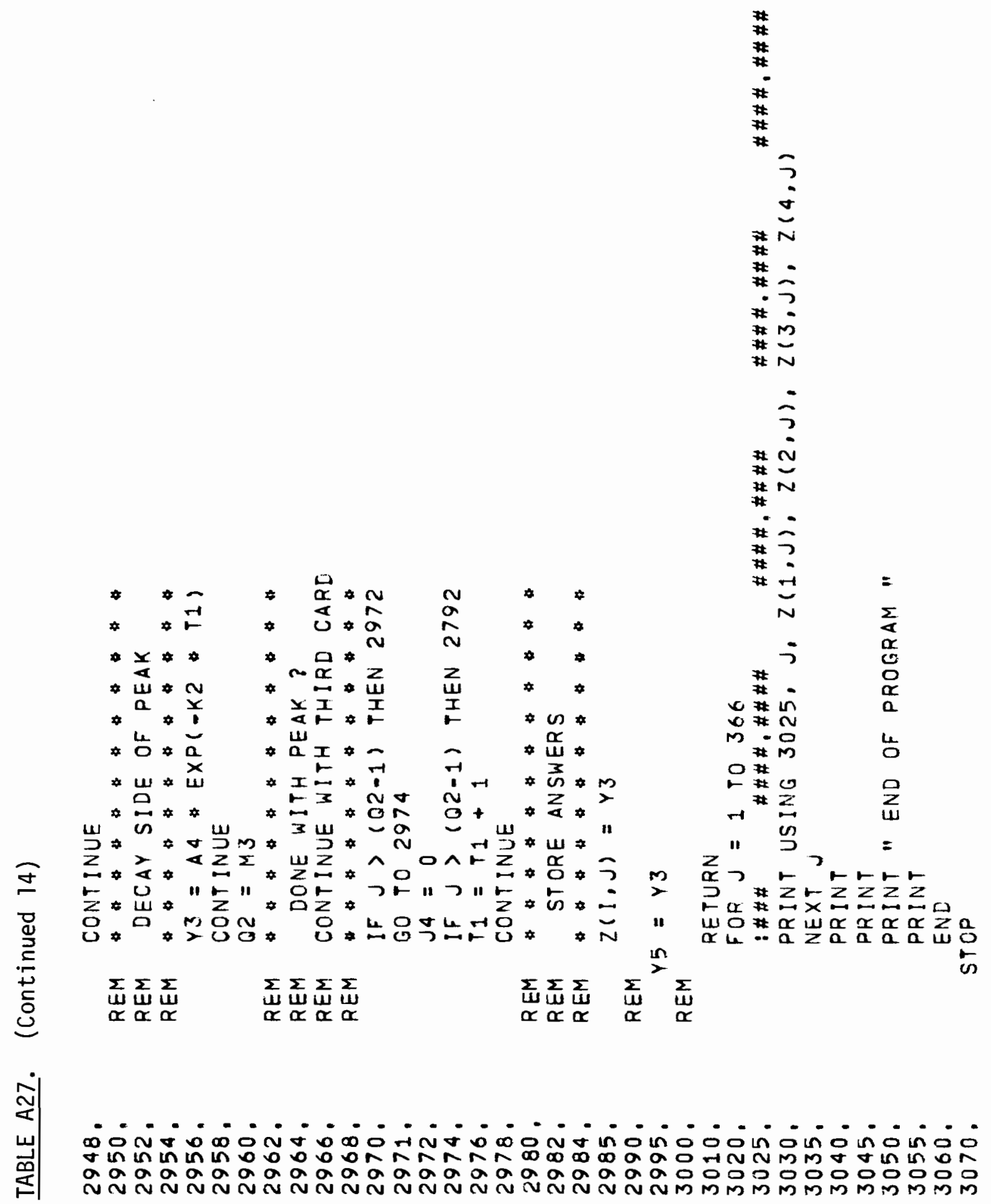



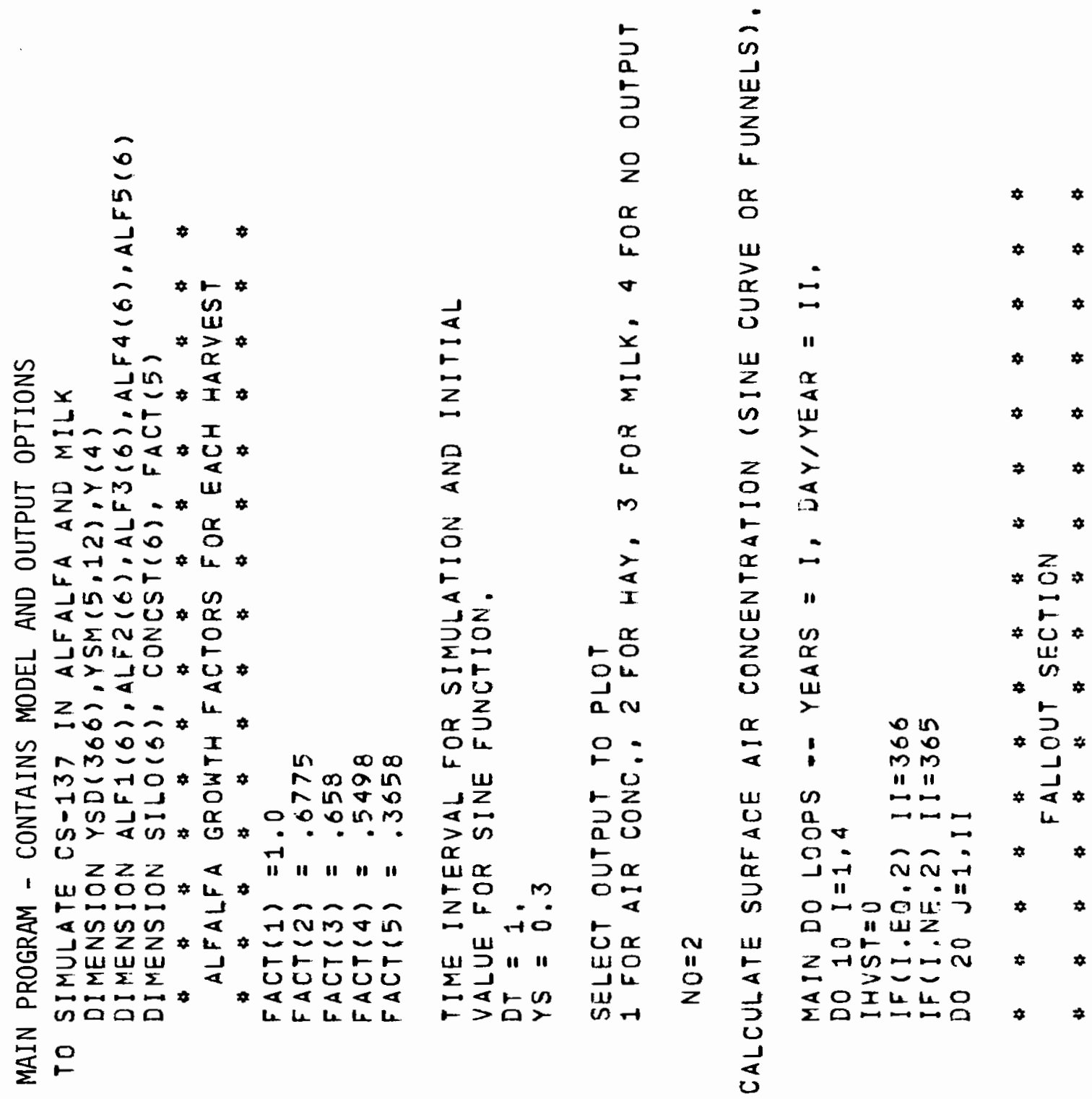


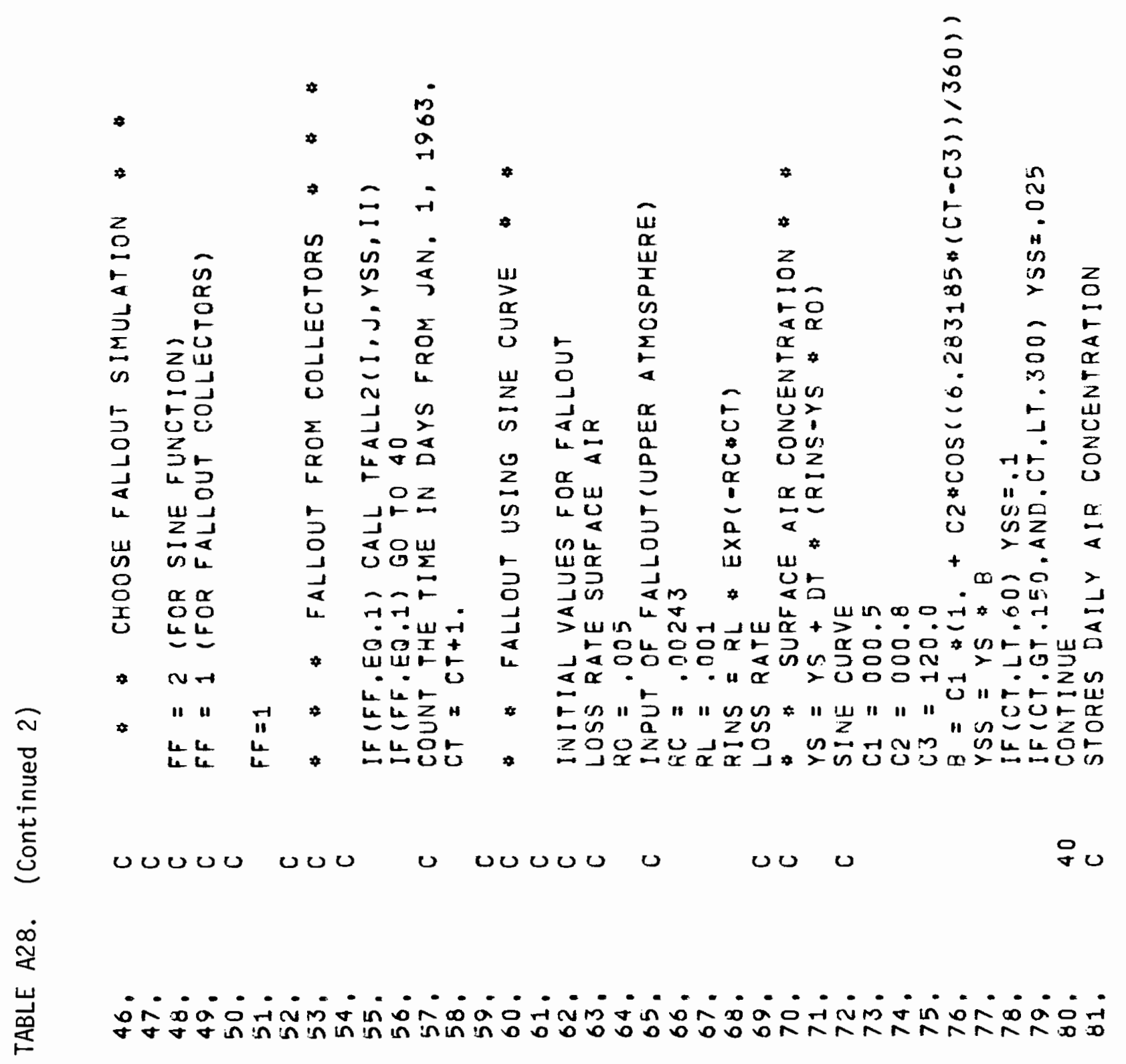




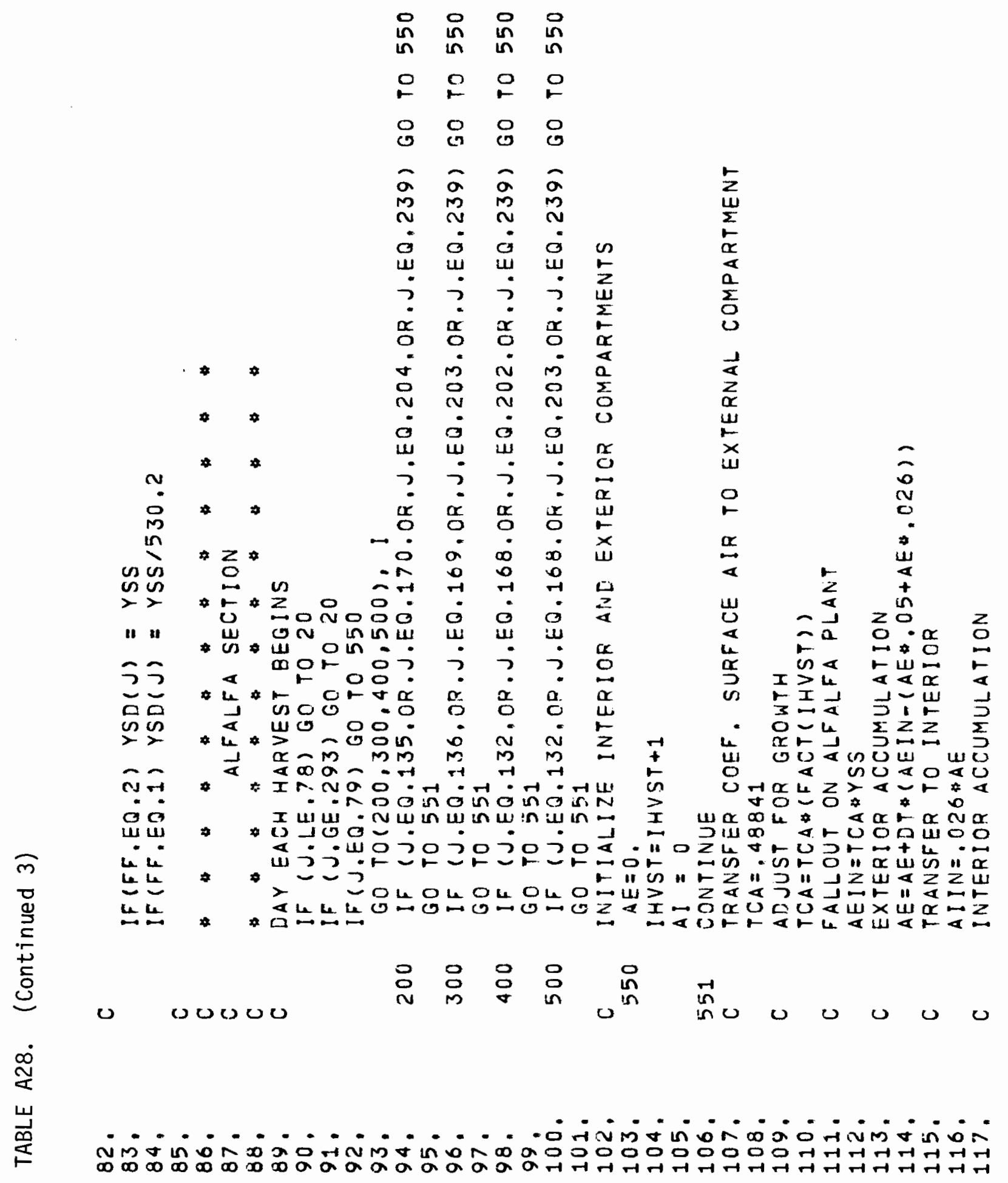




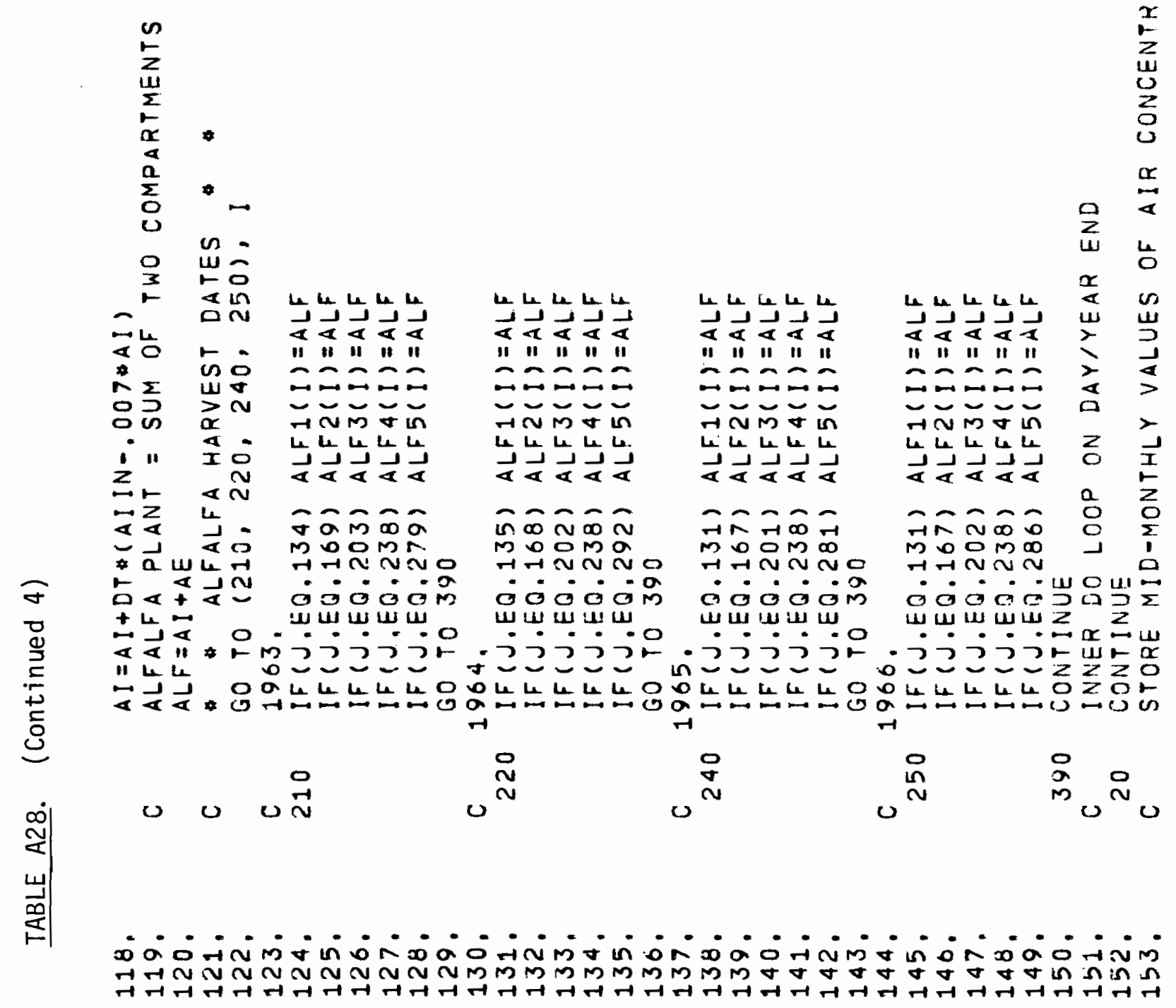




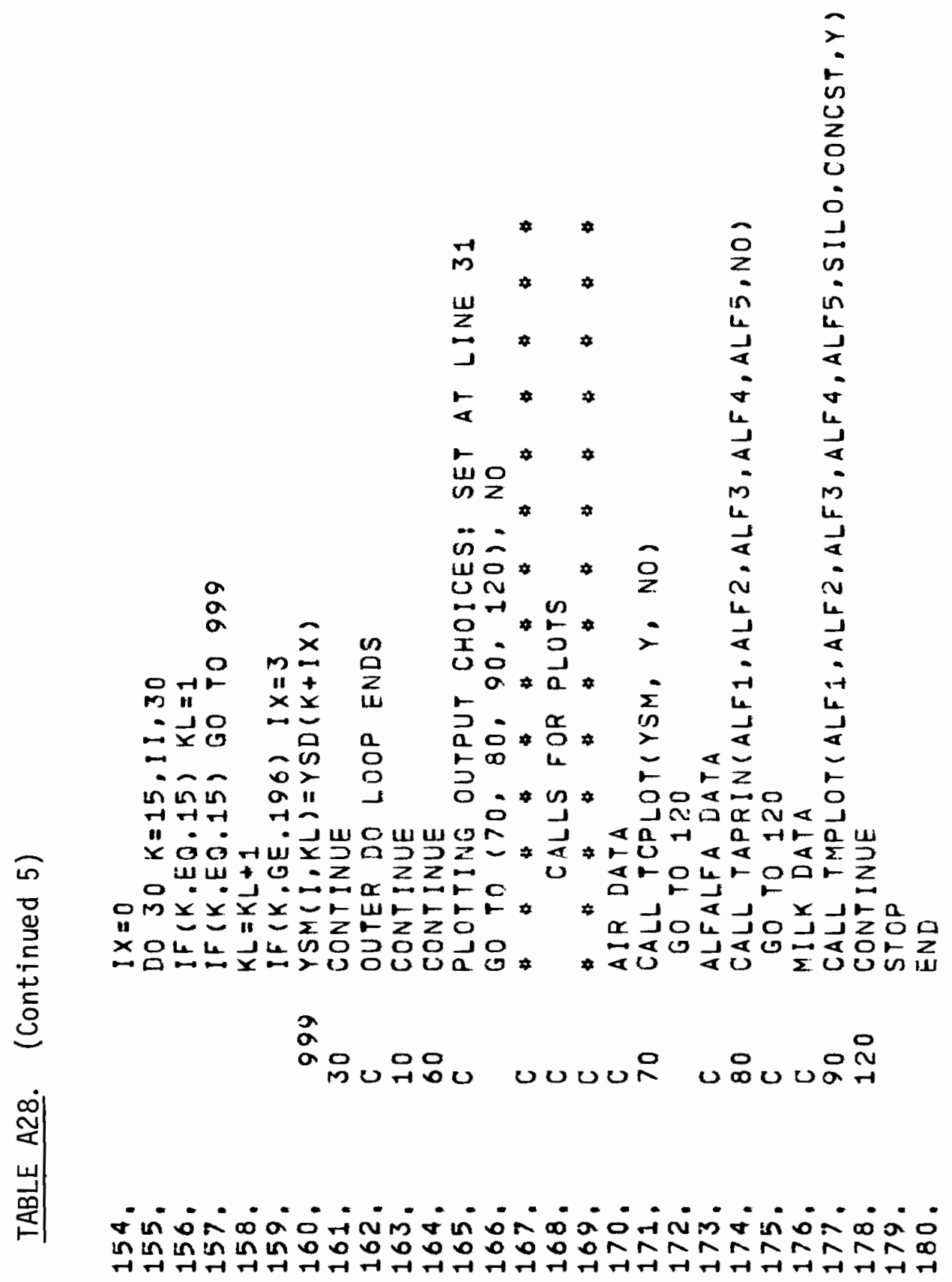



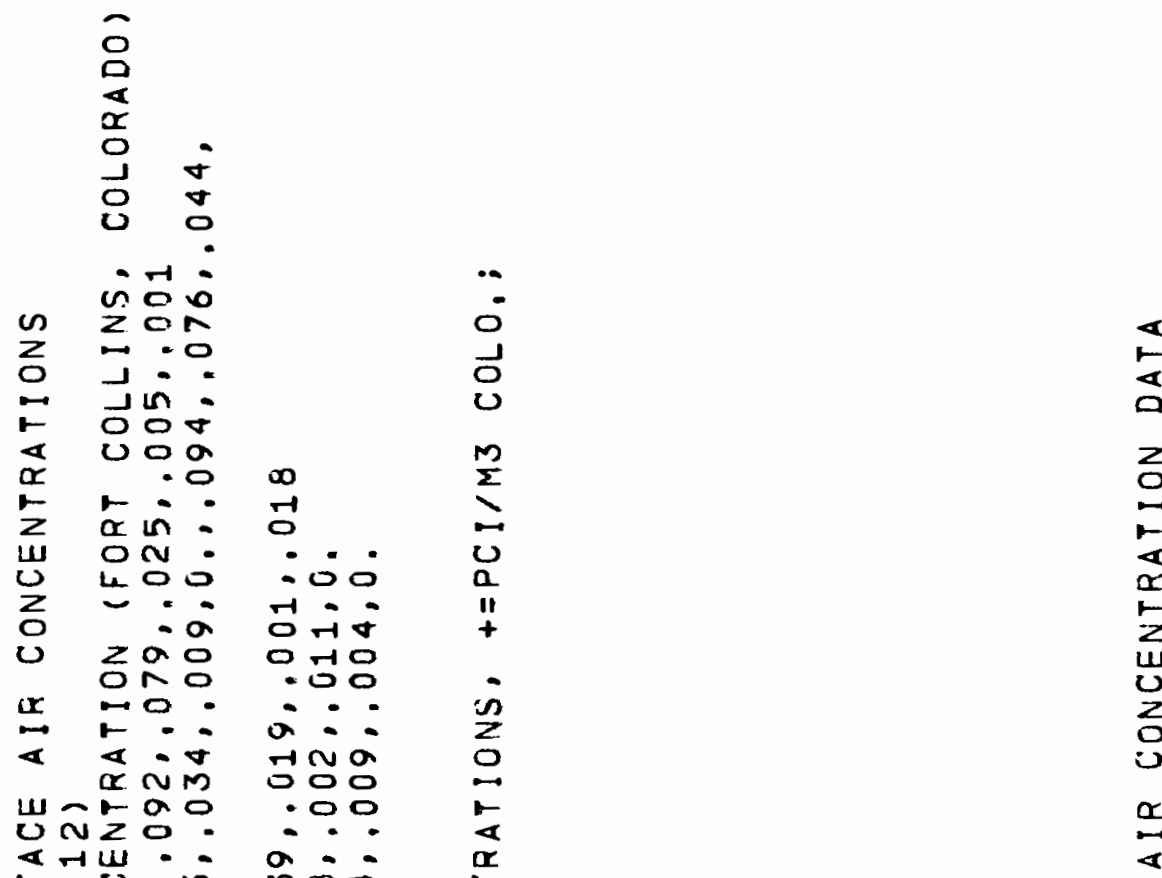

Uत⿱

तथ : $\alpha$ in $z m+$

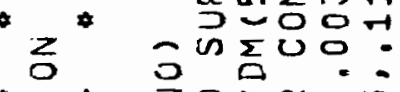

$\sum_{0}>\alpha$ : in

- $\alpha+>4=40 N$

ᄂ

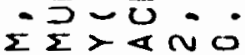

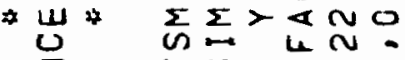

$>\omega=\mathbb{O} O N$

-

वर्त $: \overrightarrow{0}$

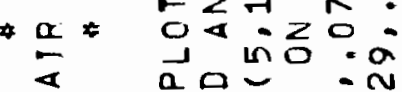

$* 4$

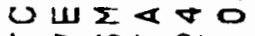

00

00

$a:-$

$m$ o d

ONM

$-00$

i: :-

m M

- 00

a.?

न : :

$0.0 \%$

- i.

- in $0 \mathrm{~m}$

$\rightarrow 000$

न०OO

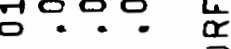

in iो

anol 0

न100

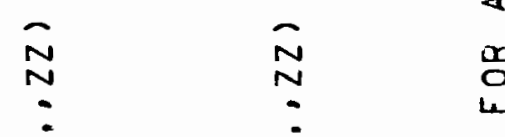

$a$
4
0
2
0
0
$\alpha$
2
2
2
0
0

$\frac{1}{4}$

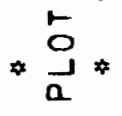

$\overrightarrow{x>a n:}$

$\therefore:-i 0 x$

ifiog

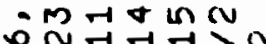

w山 $z^{0}-0$

०NनHA

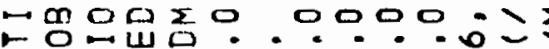

Mnia is

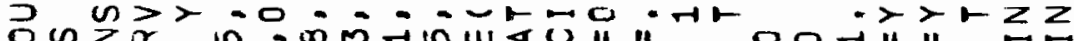

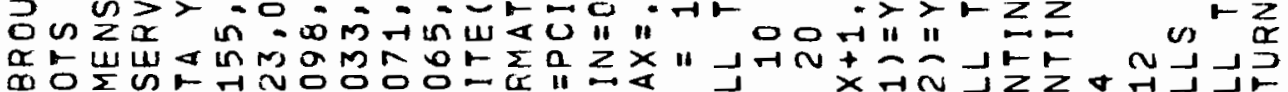

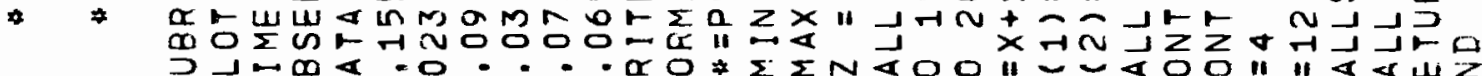

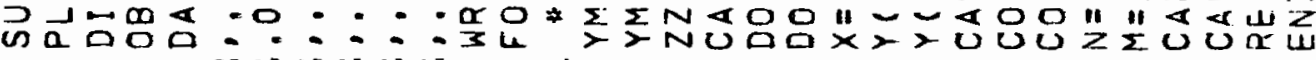

$\infty$
$y$
$x$
$\frac{1}{\infty}$
$\frac{1}{-1}$

$\ln _{0}^{0}$

in

in

क्वं

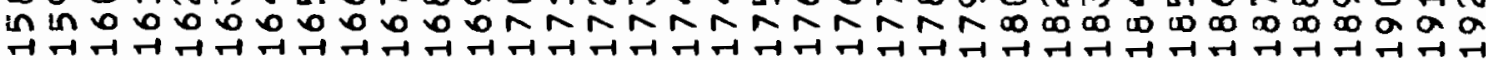




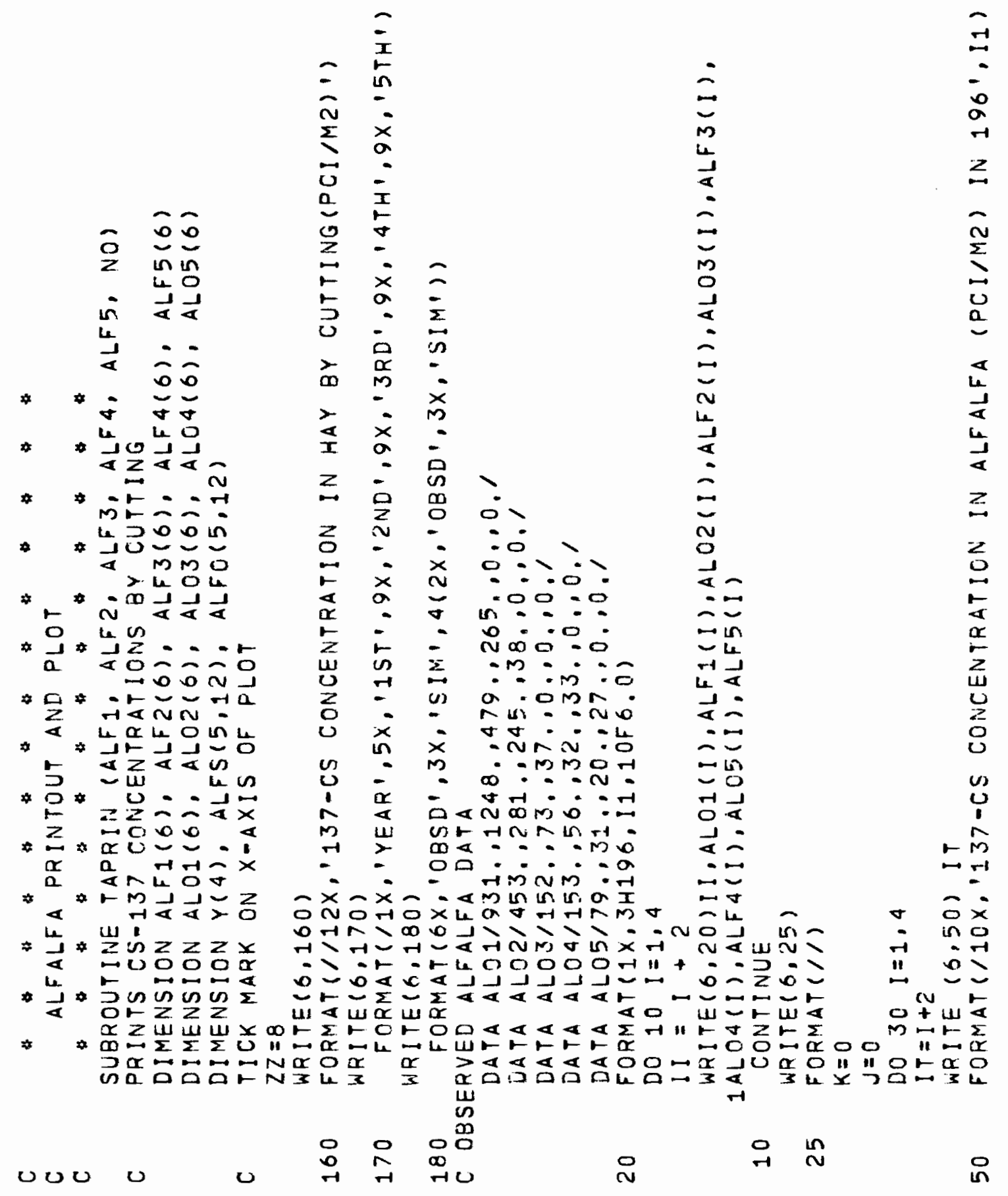

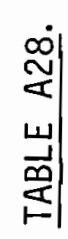

in in $\ldots \ldots \ldots$. . . . . . . . . . . . . . .

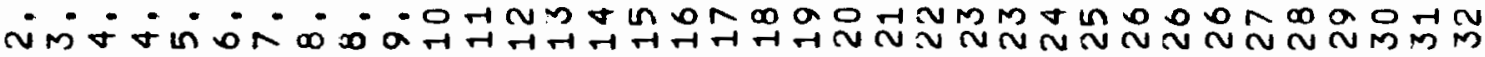




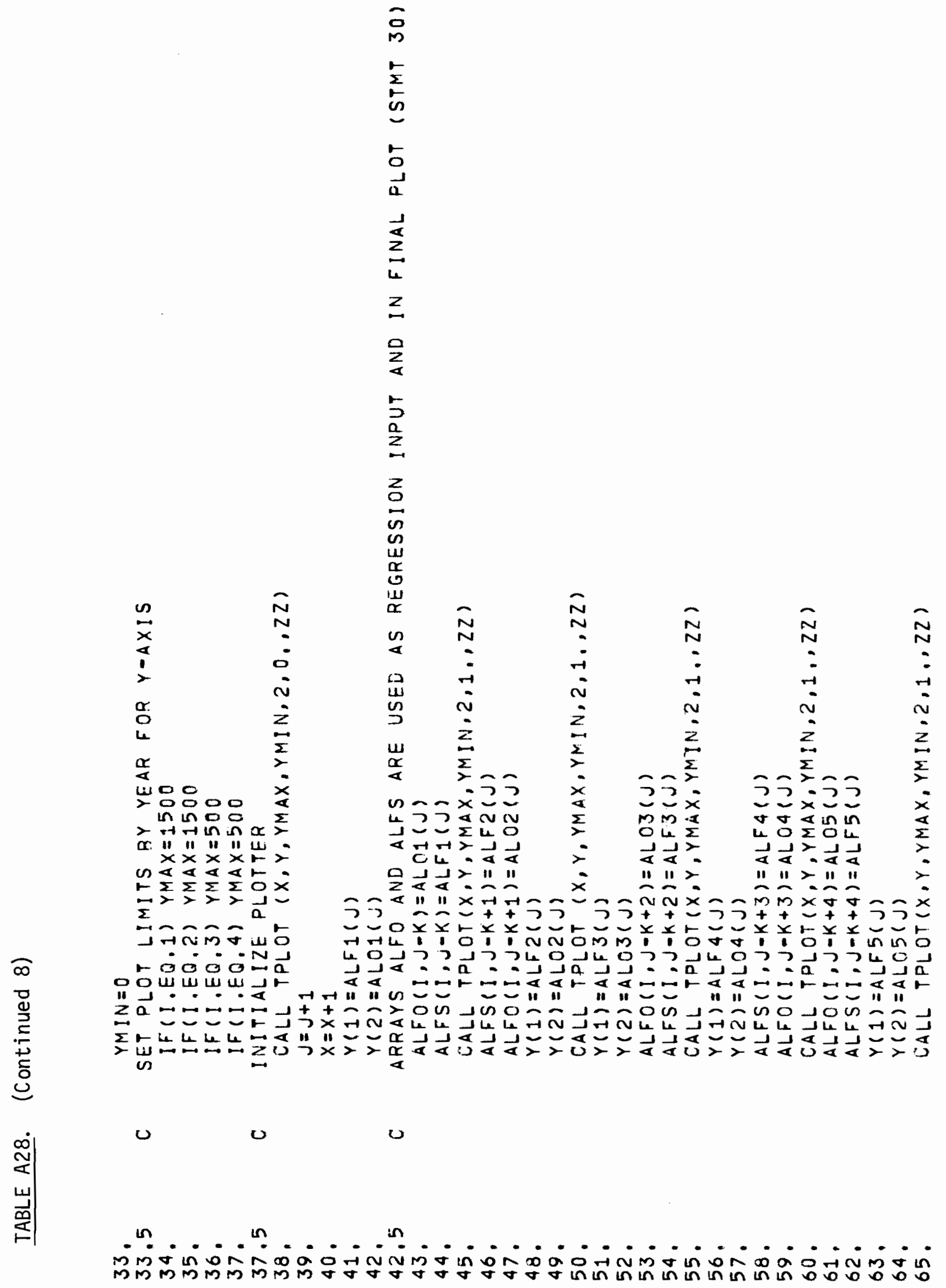




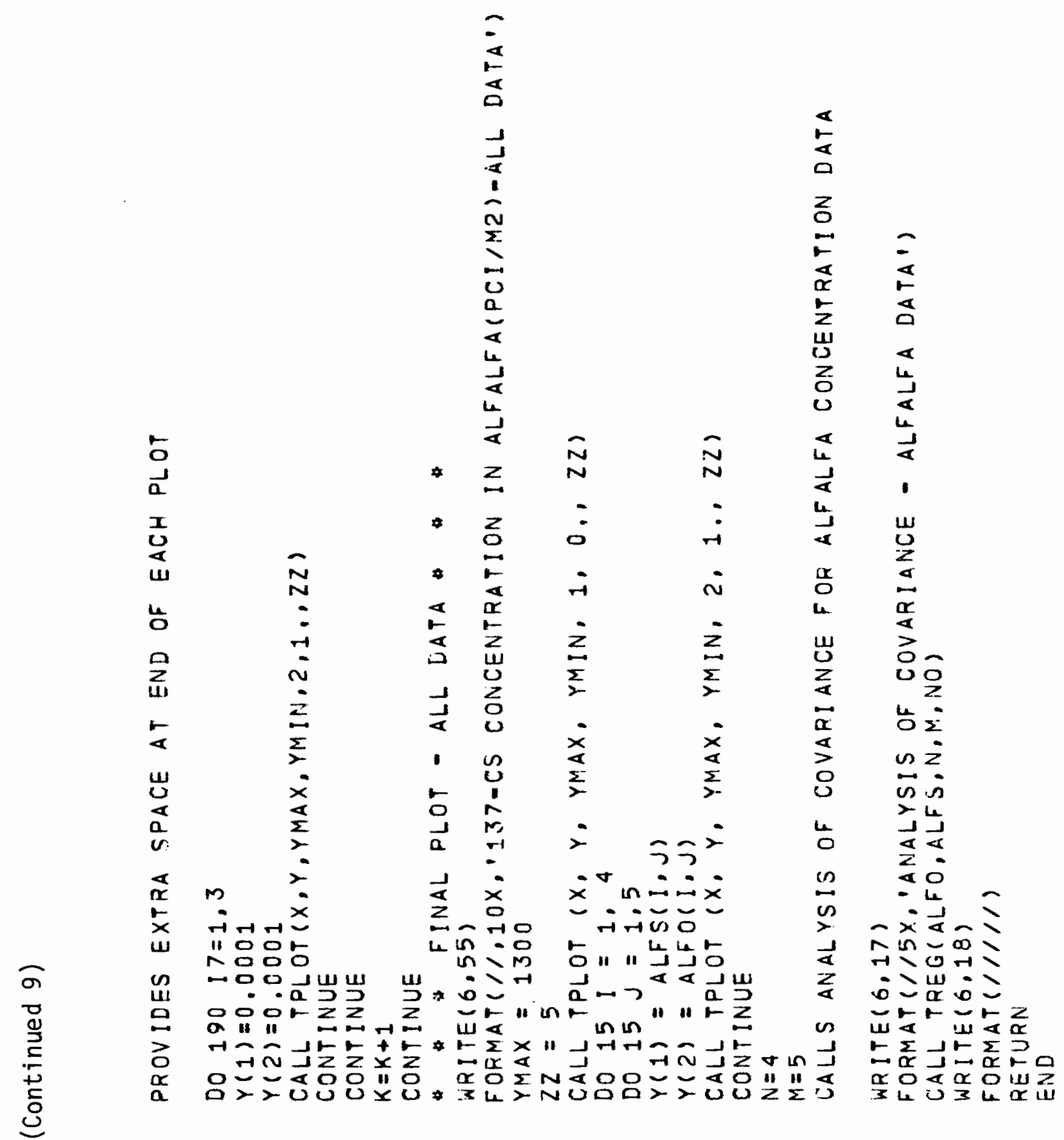

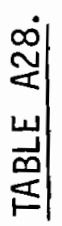

$\underset{\substack{0 \\ \hdashline}}{0}$

○ $\ln _{4}$

$00 \stackrel{\rightarrow}{n} \stackrel{\infty}{-1}$

no...... ğ in ON ó 
* 00 in

*

- in -

2

in

$\because \quad$ n -0

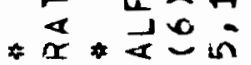

a $\quad 0$

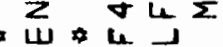

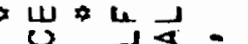

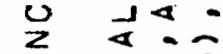

*

4

* $x \neq I m$

$\Rightarrow \quad 4=$

$\neq \vec{\Sigma} \sim \vec{\sim} \dot{0}$

( $)=$

*t aci

o $-0 u$

* $\vec{a} *$ in 2

- $\frac{1}{4} 10$

* $\sum_{a}+2$

-

* cos 00

는

* $45 \frac{1}{4}$

马

* 0 * $2 z z$ $m 00$

$n$
0
-1

in

$\ln$
0
0
0

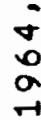

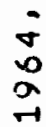

mo

m

a

$\mathbb{r}$
0

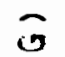

$\underset{x}{0} \quad \stackrel{0}{x}$

ㄴ.

$\underline{\mathbf{a}}$

w

a

0
$\vdots$
0
$a$
$a$
$z$

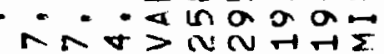

แกヘ。 J" 11 "

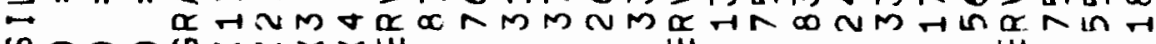

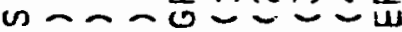

$\begin{array}{ll}3 & 0 \\ 0 & 0 \\ 0 & 0\end{array}$

*

$002 z$

" U1" $11 " 11 "$ " 11 " oxiv

- $\quad \frac{1}{0} \Sigma \frac{1}{\Sigma} \frac{1}{2}$

a

HNm

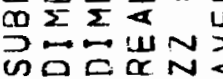

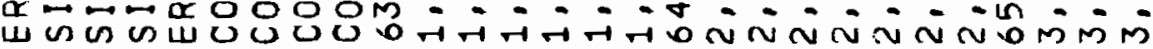

$\infty$
$\frac{1}{c}$
$\frac{1}{0}$
$\frac{1}{r}$

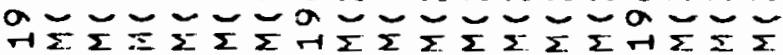

UU

0

ט

U

v

0

\section{in}

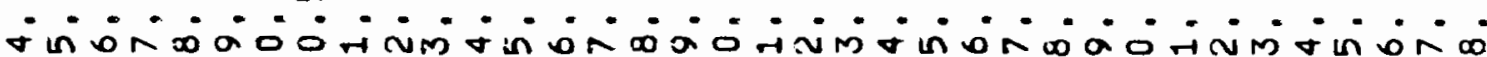

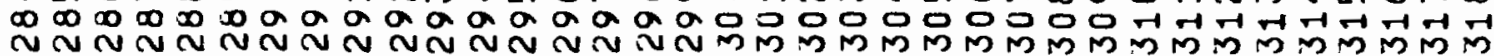




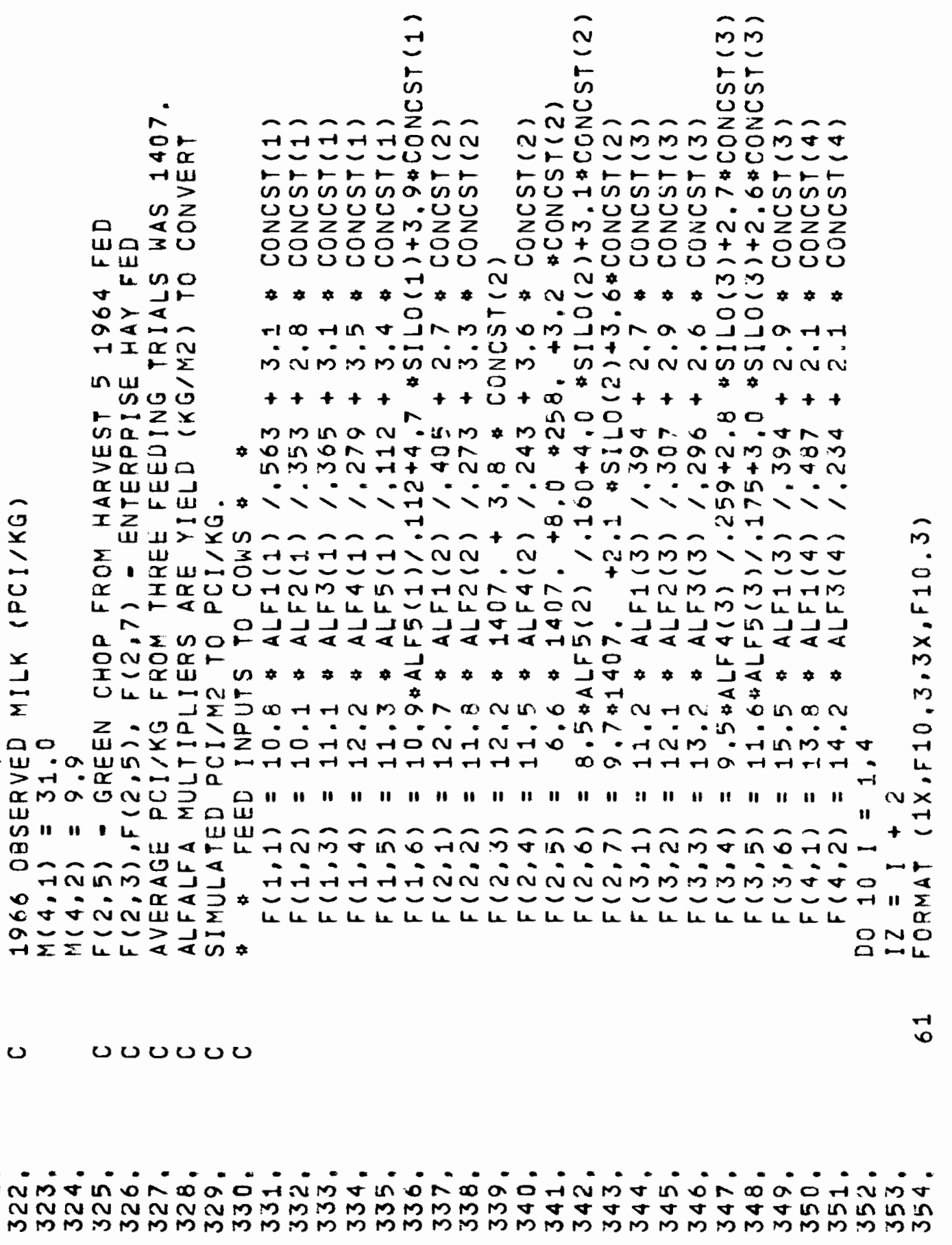

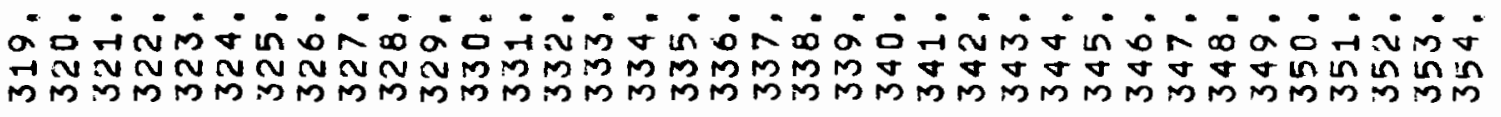




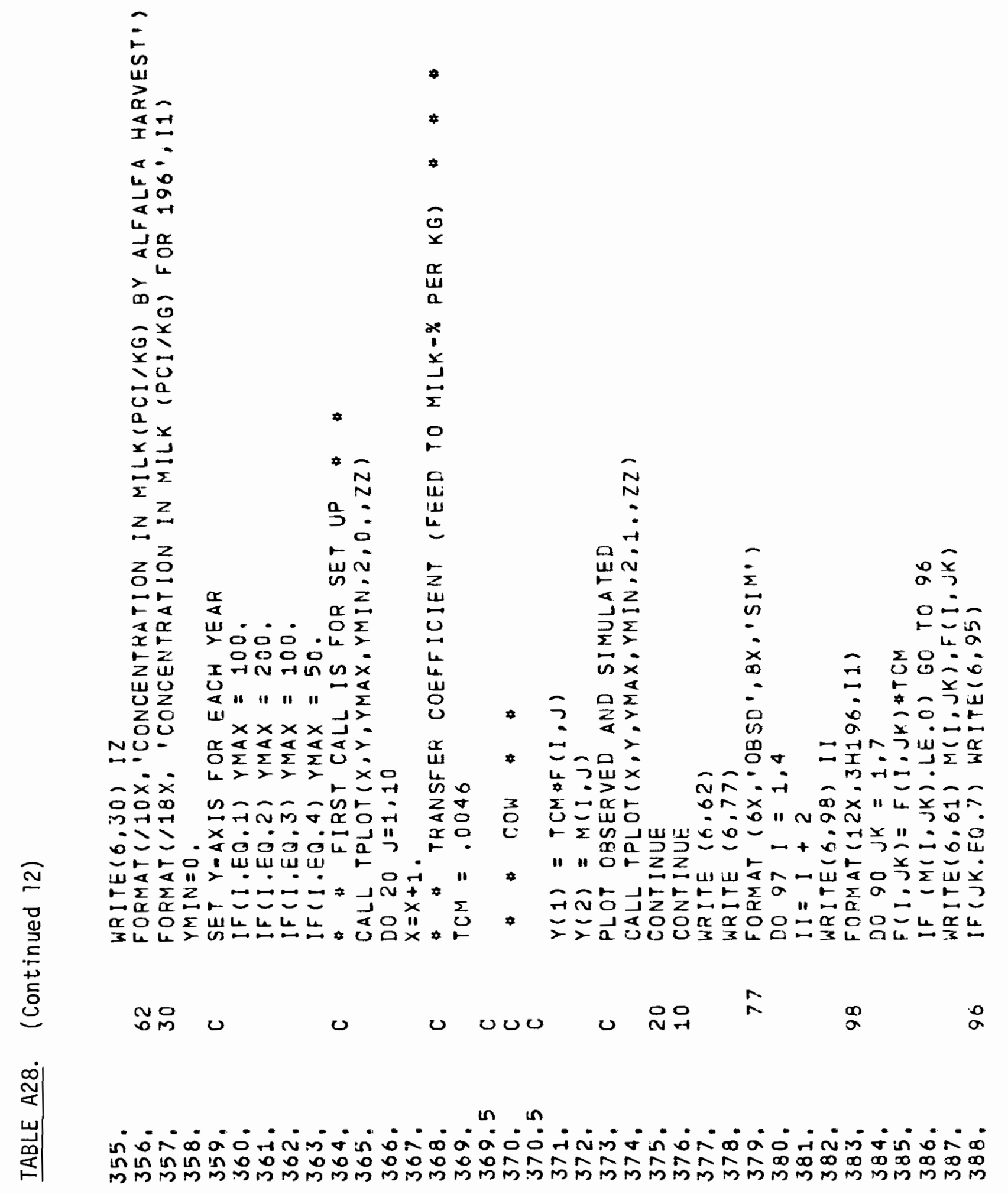




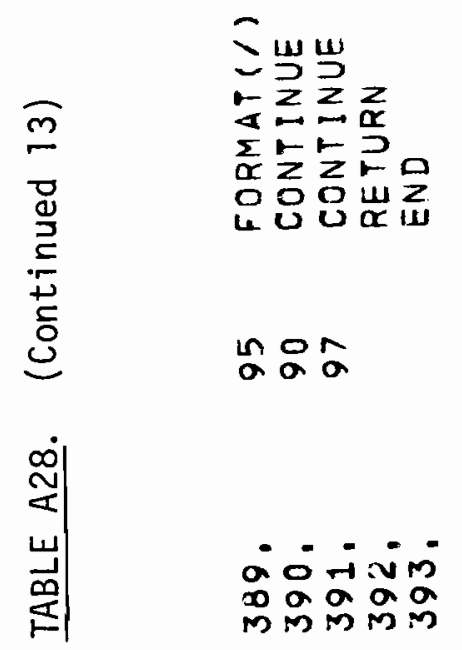

A. 117 


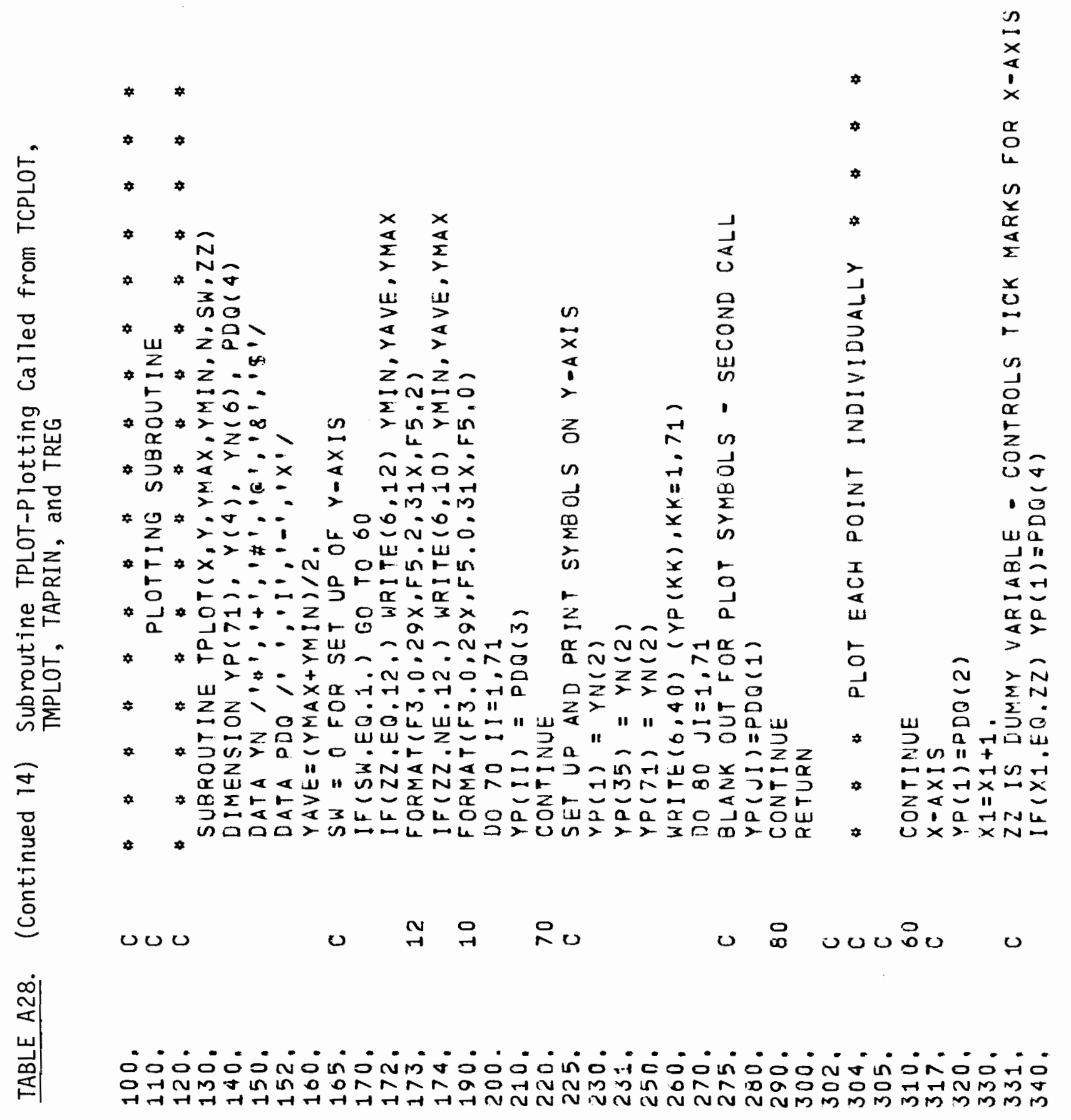




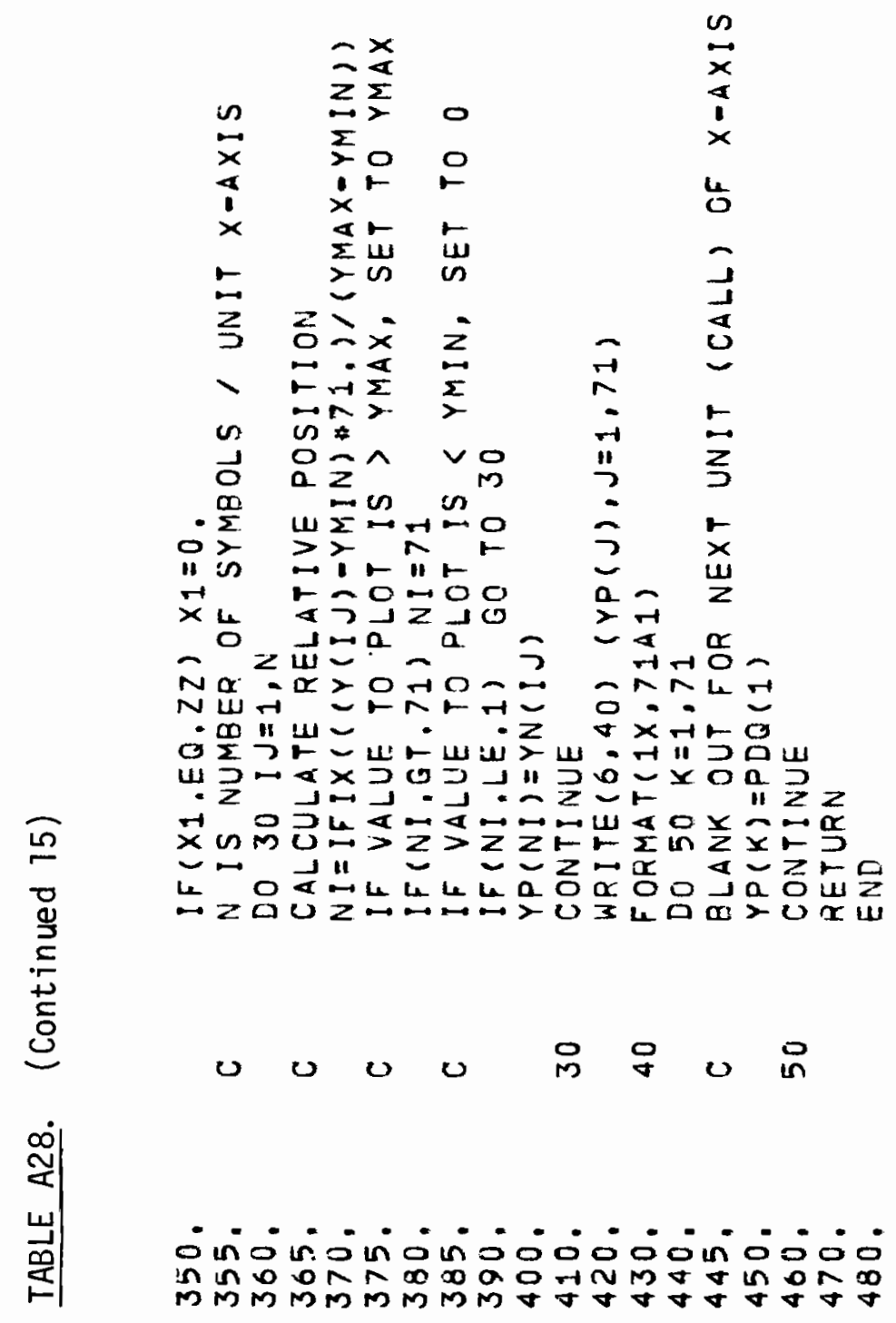




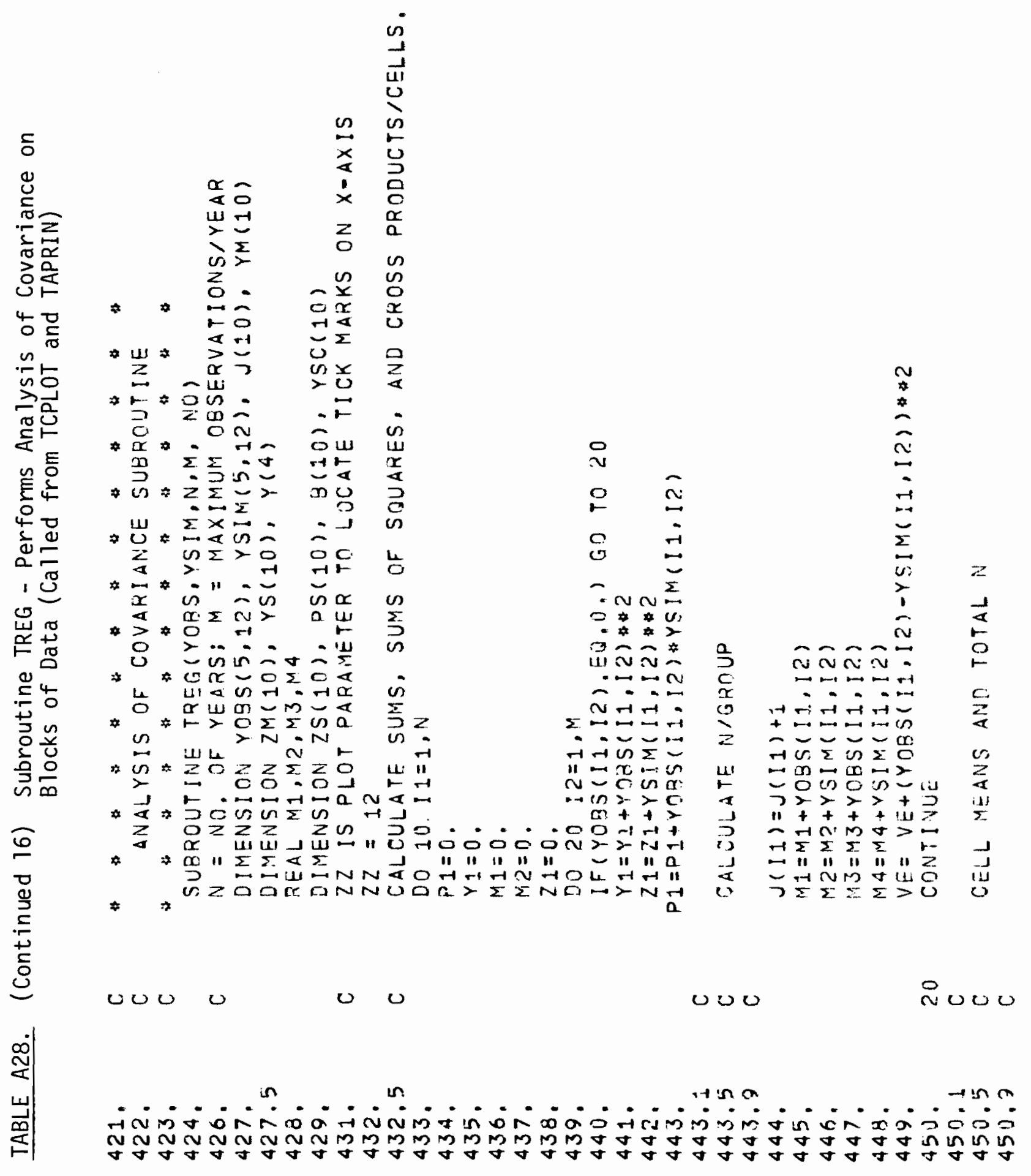




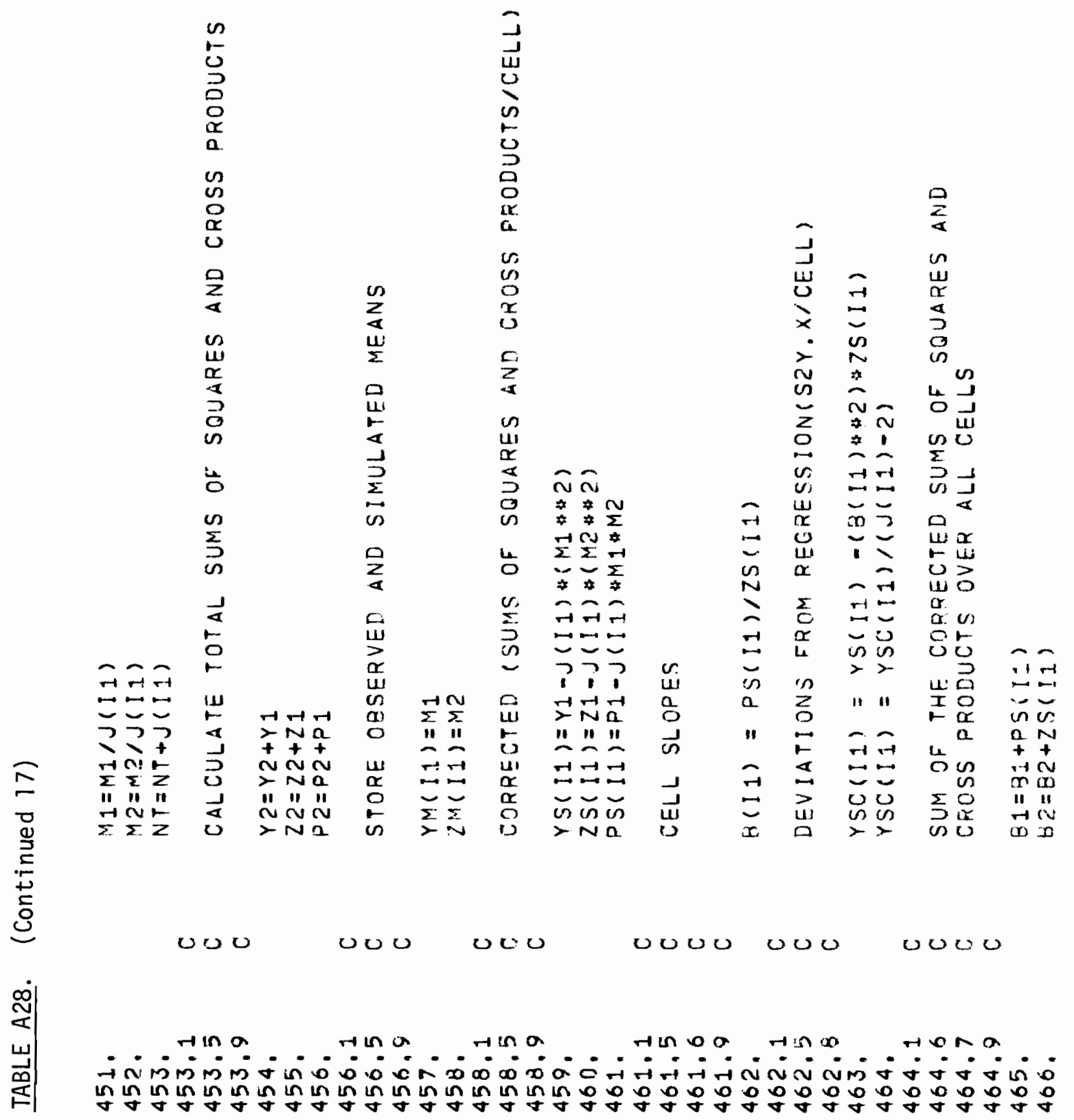




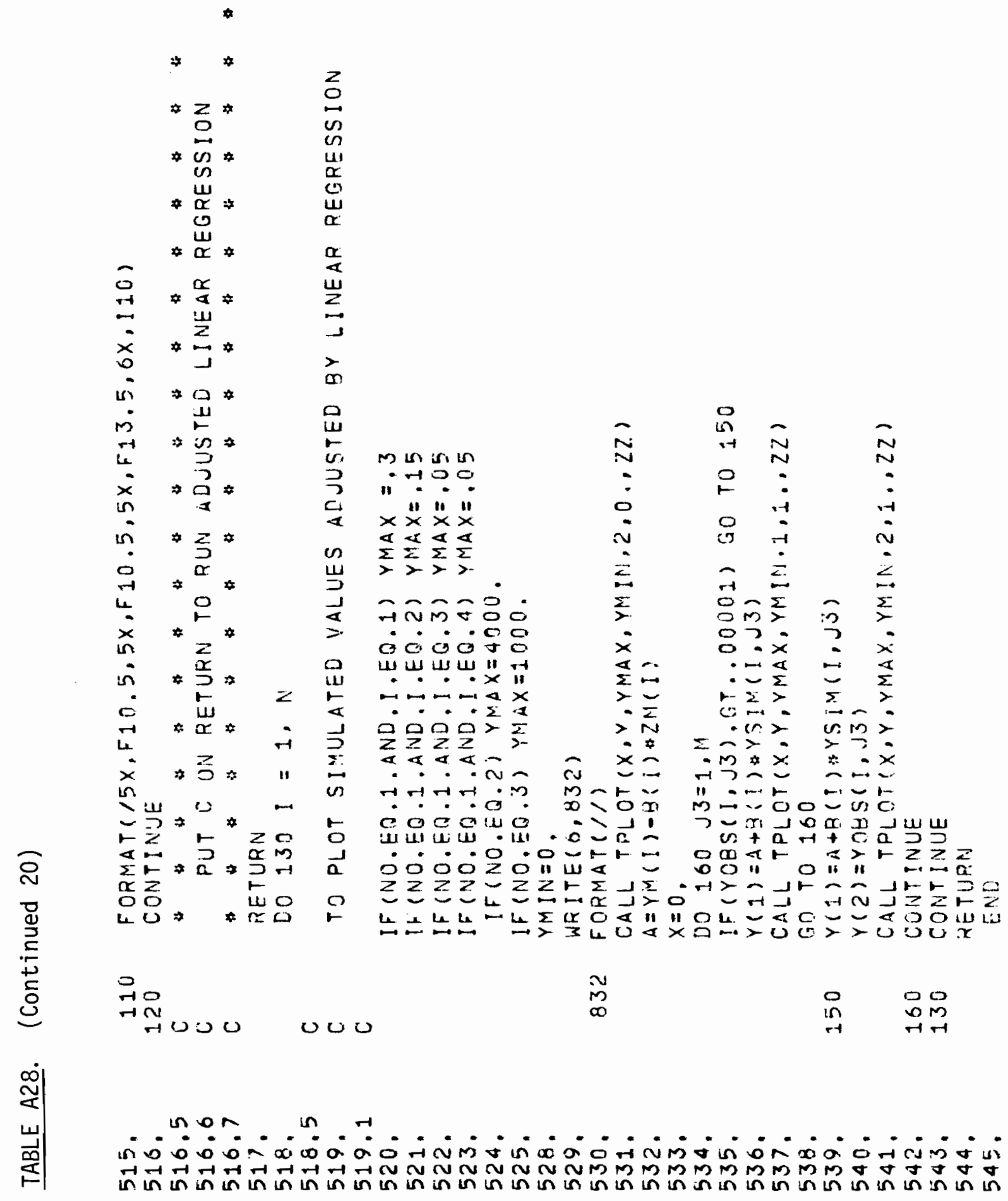




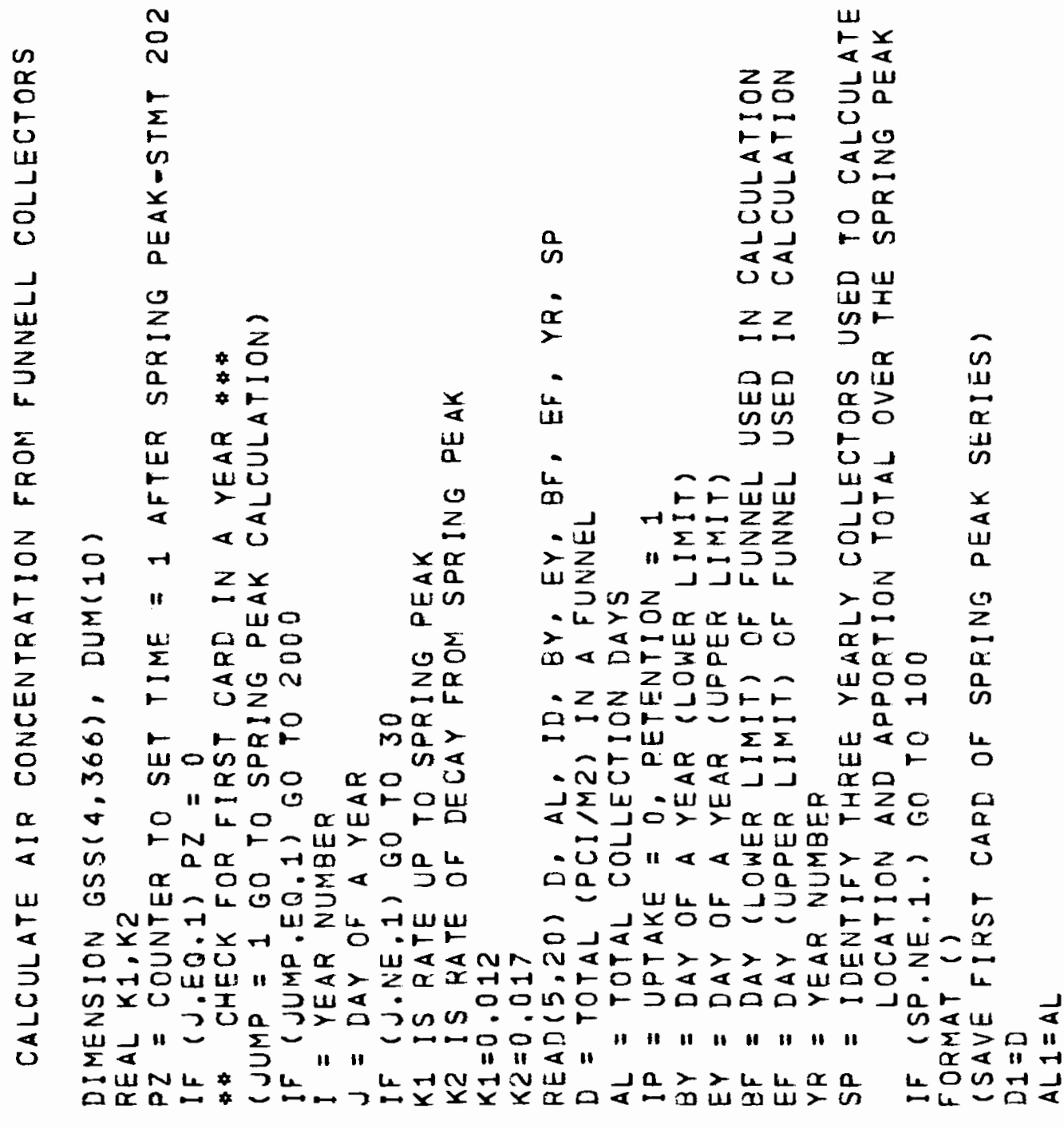

ט

$\circlearrowleft$

000000

ㅁov0000000

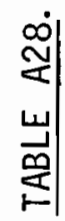

-

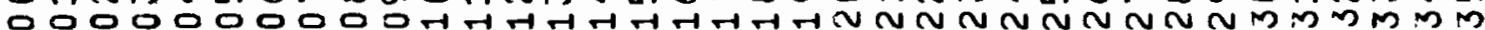

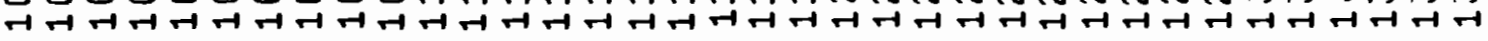




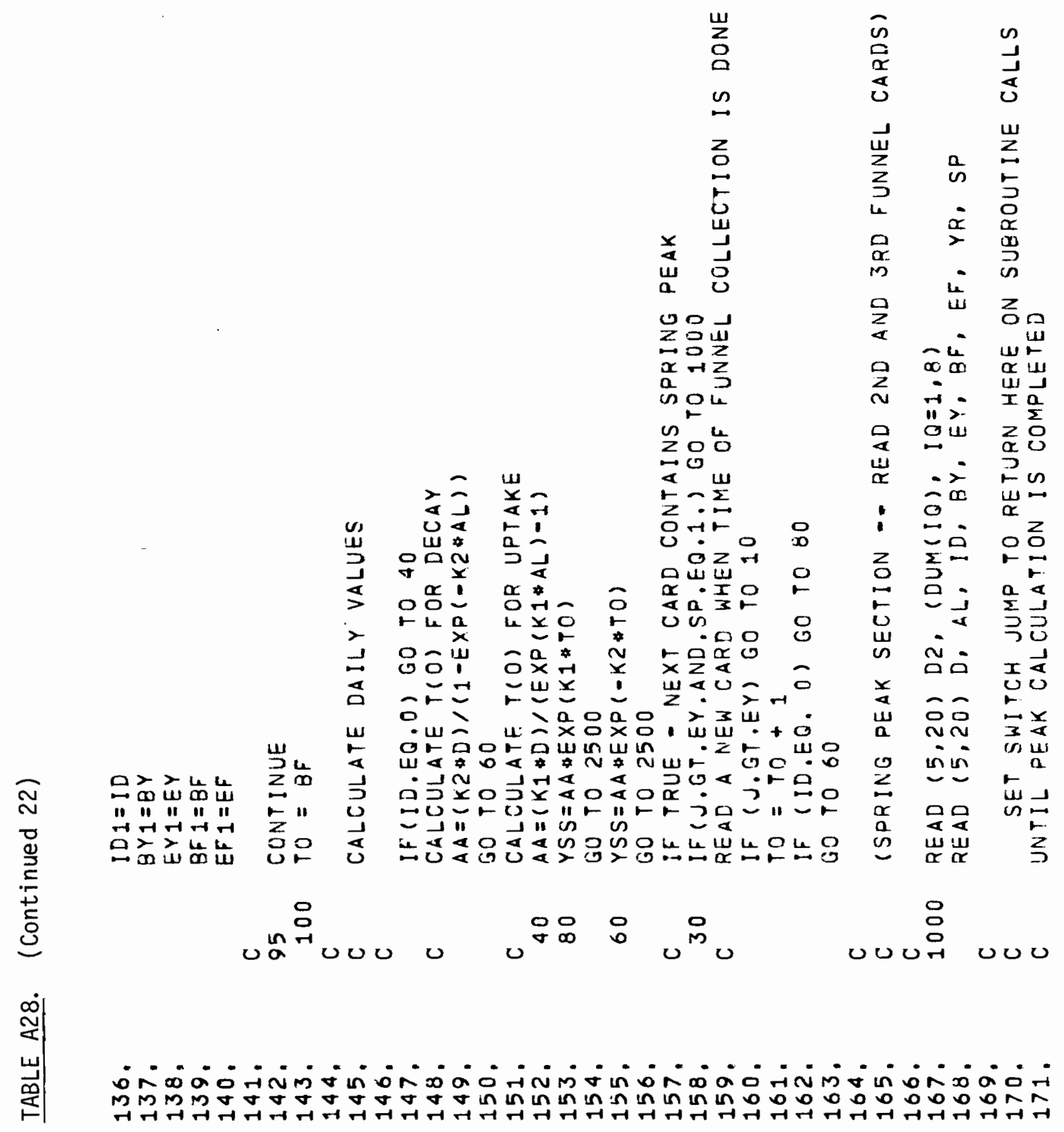




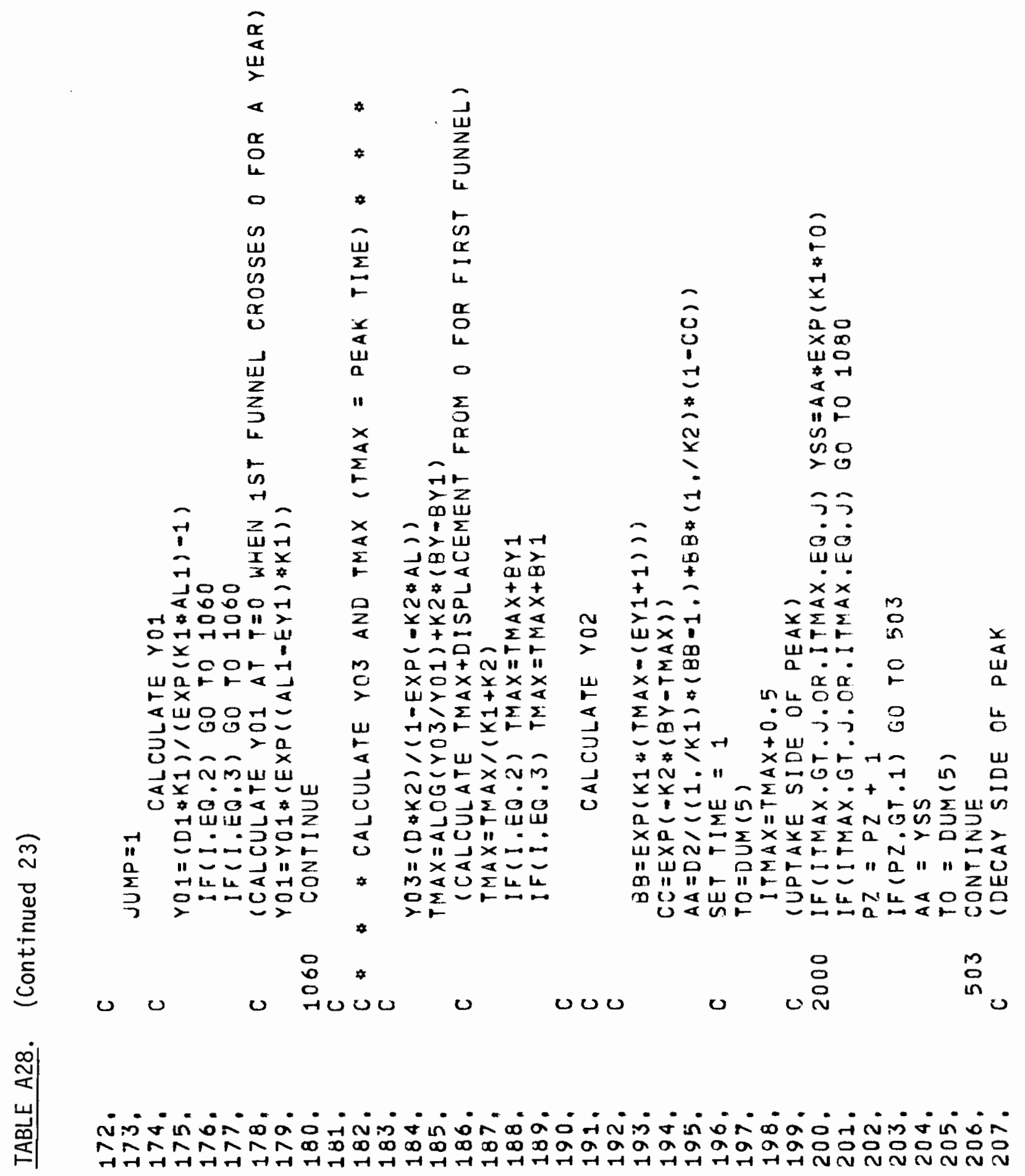




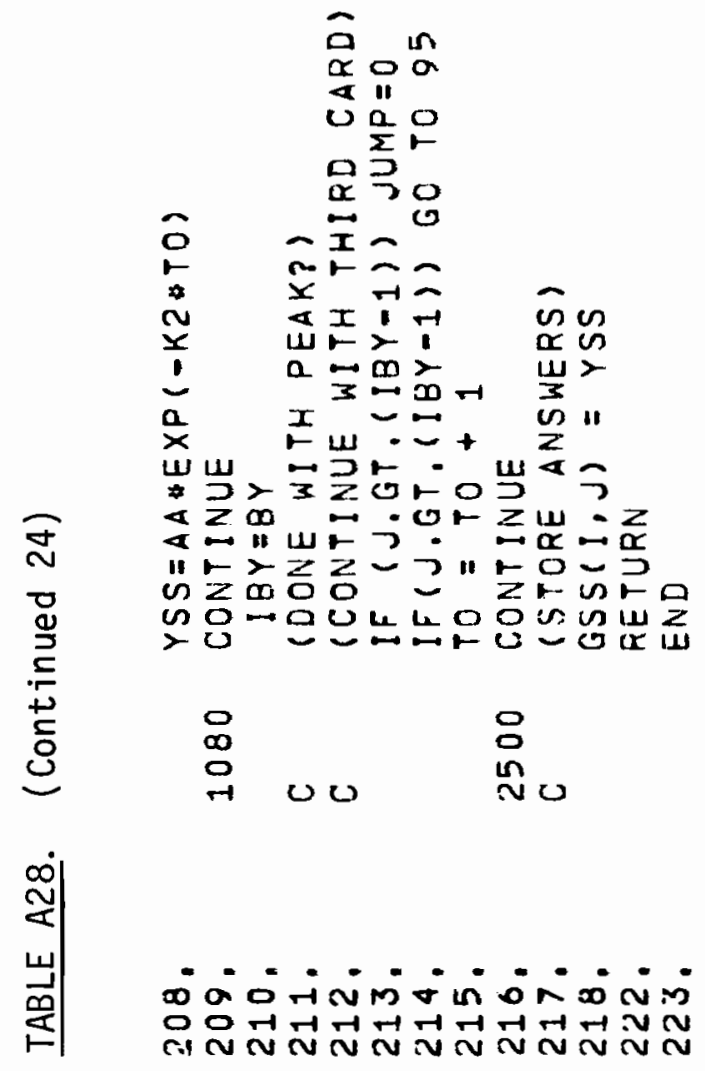

A. 128 
TABLE A28. (Continued 25) Data File TEMP - Contains ${ }^{137} \mathrm{Cs}$ Data from Funne1s (See subroutine TFALL for Column Labels)

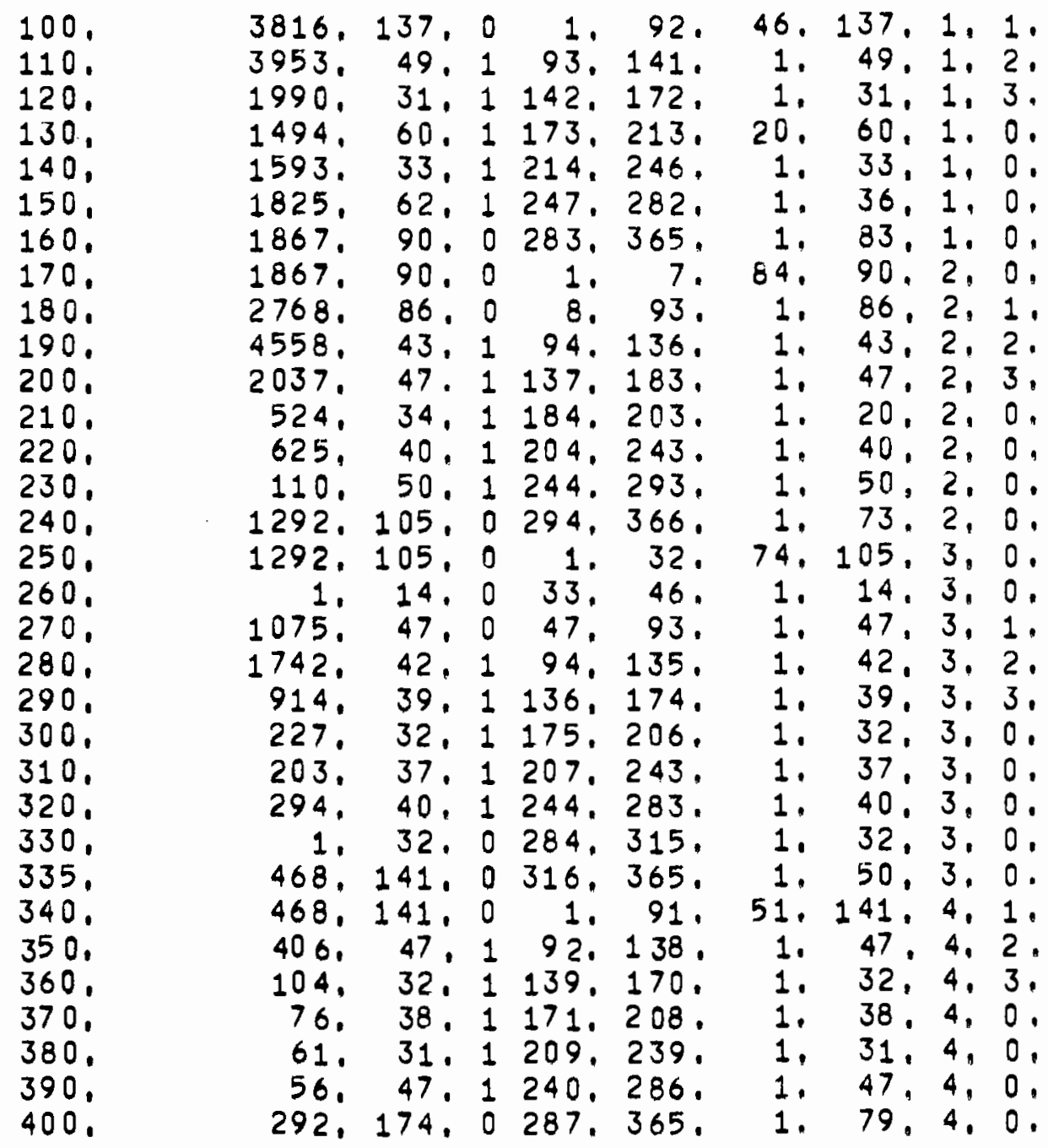




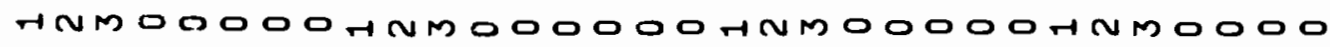

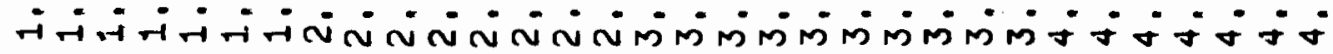

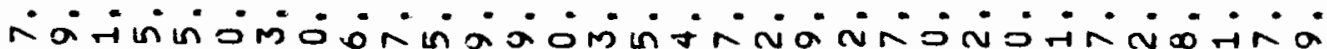

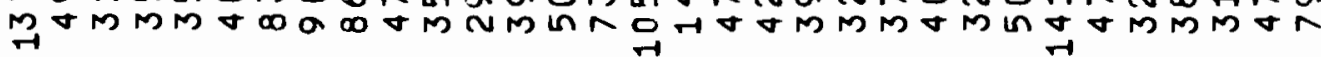

$\overline{\mathrm{O}}$

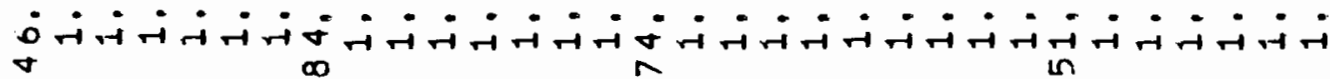

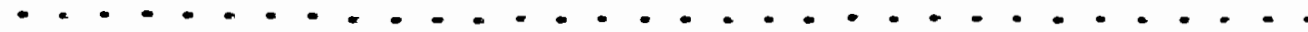

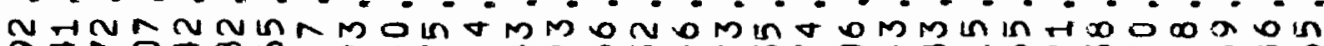

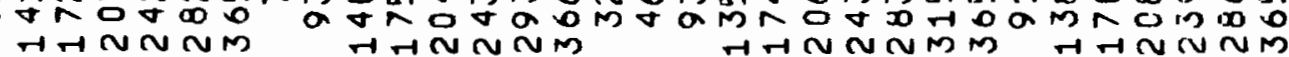

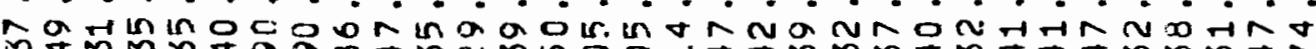

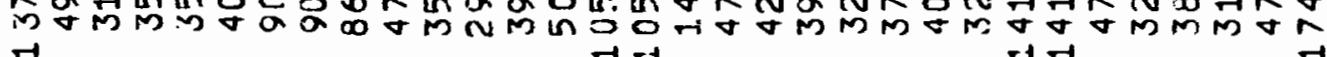

N

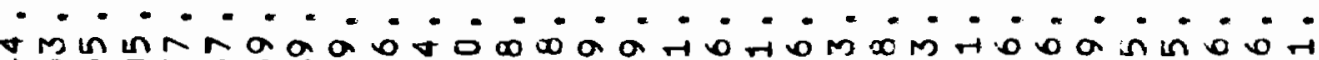

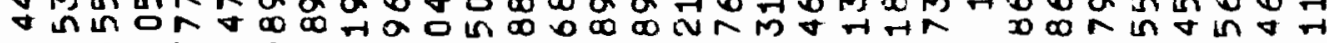

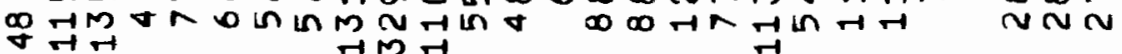

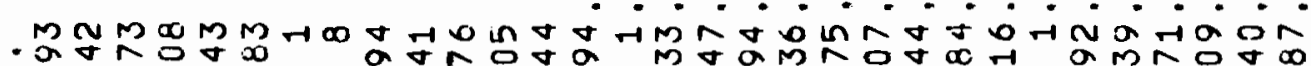

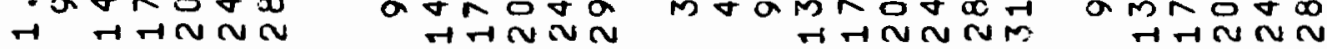

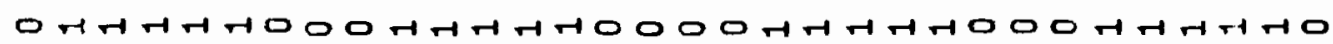




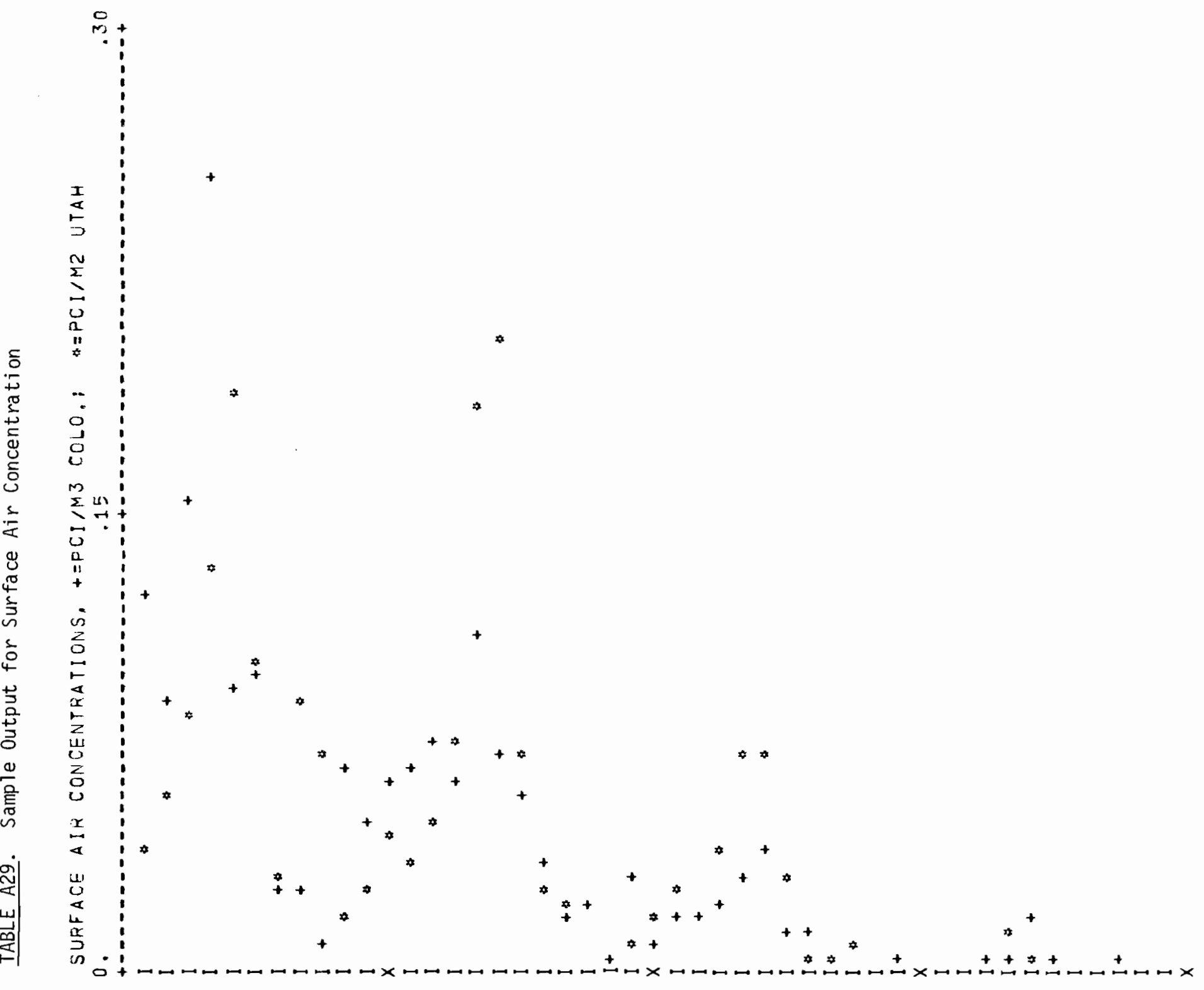

A. 131 


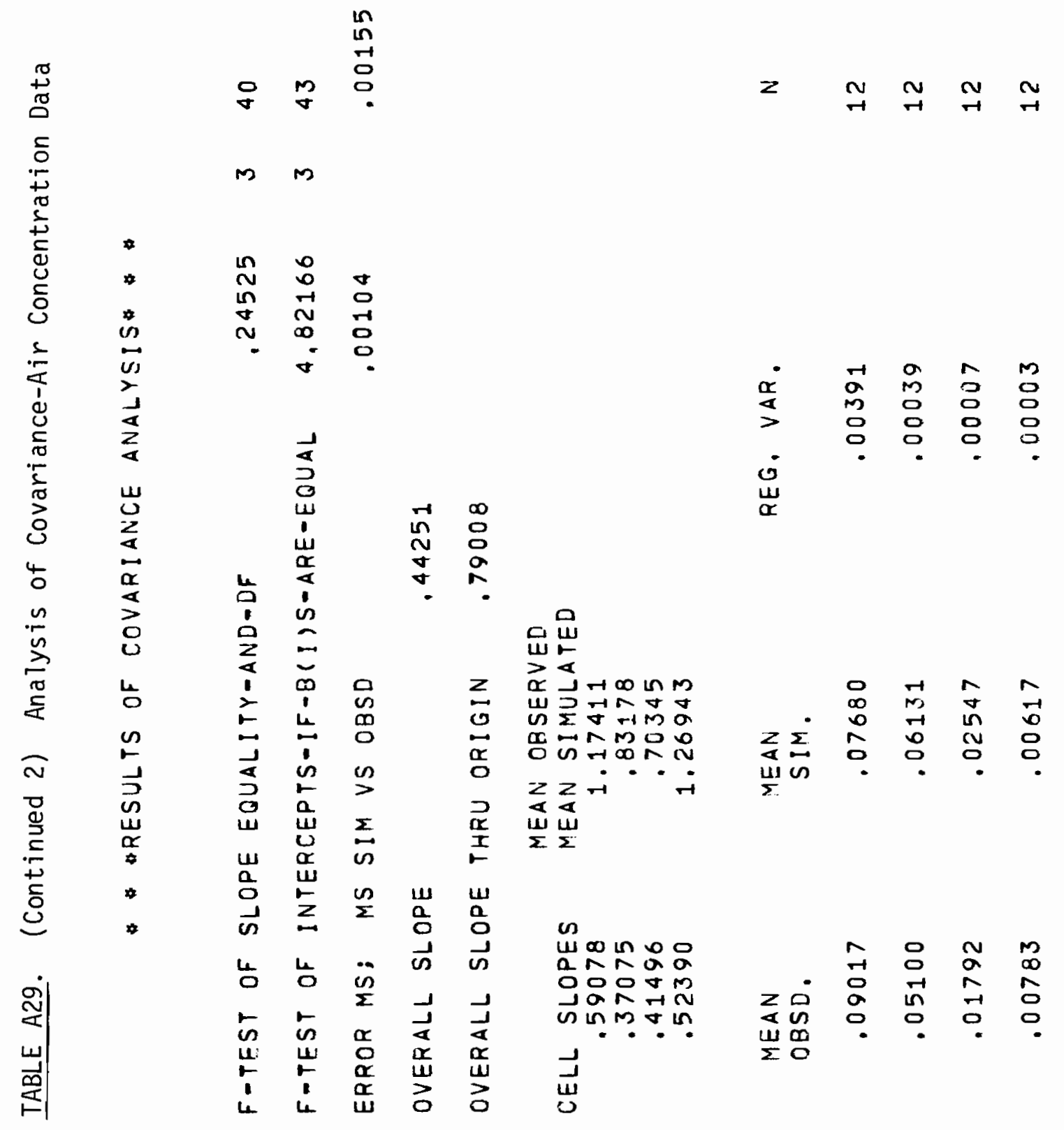




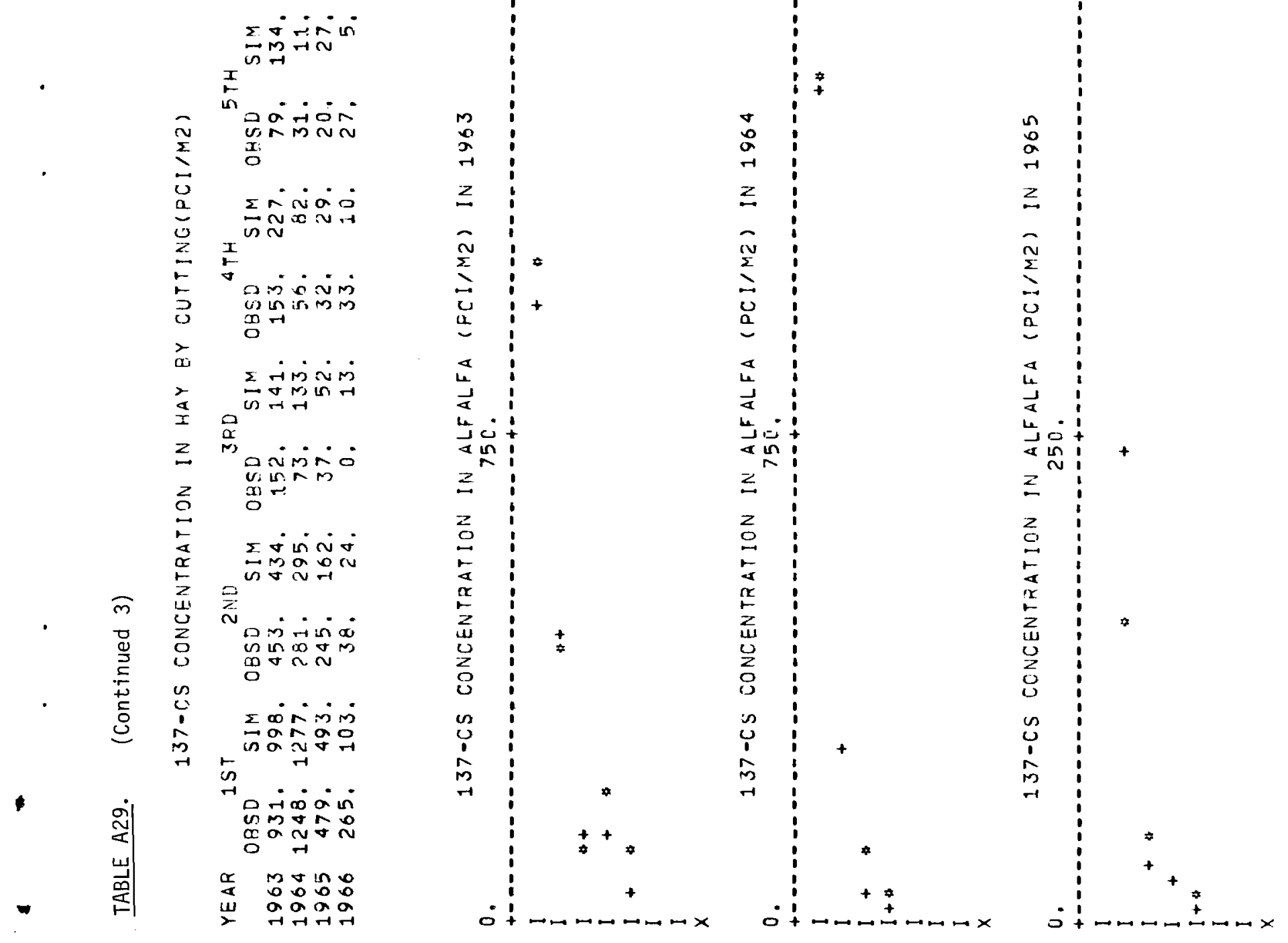



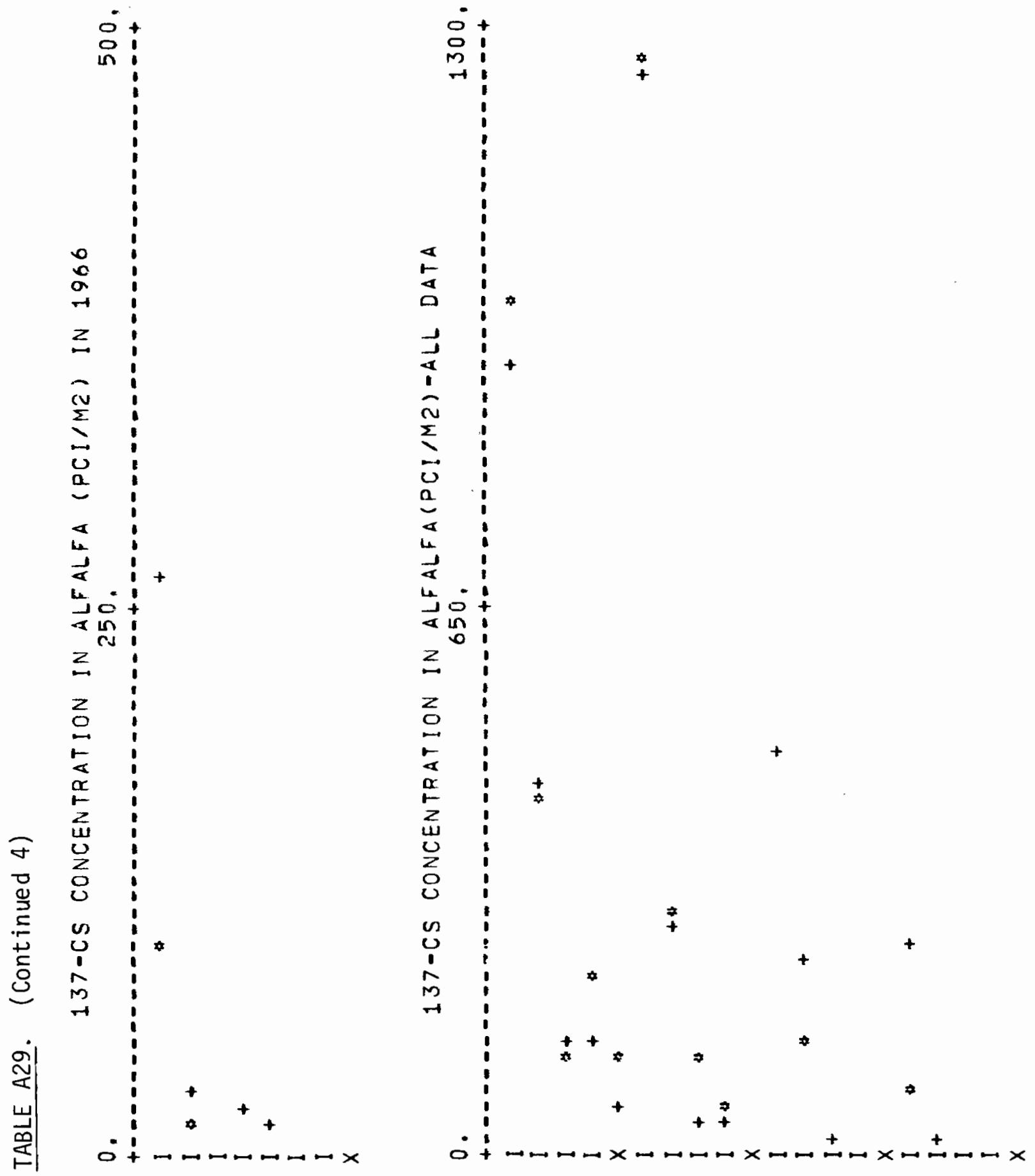

A. 134 


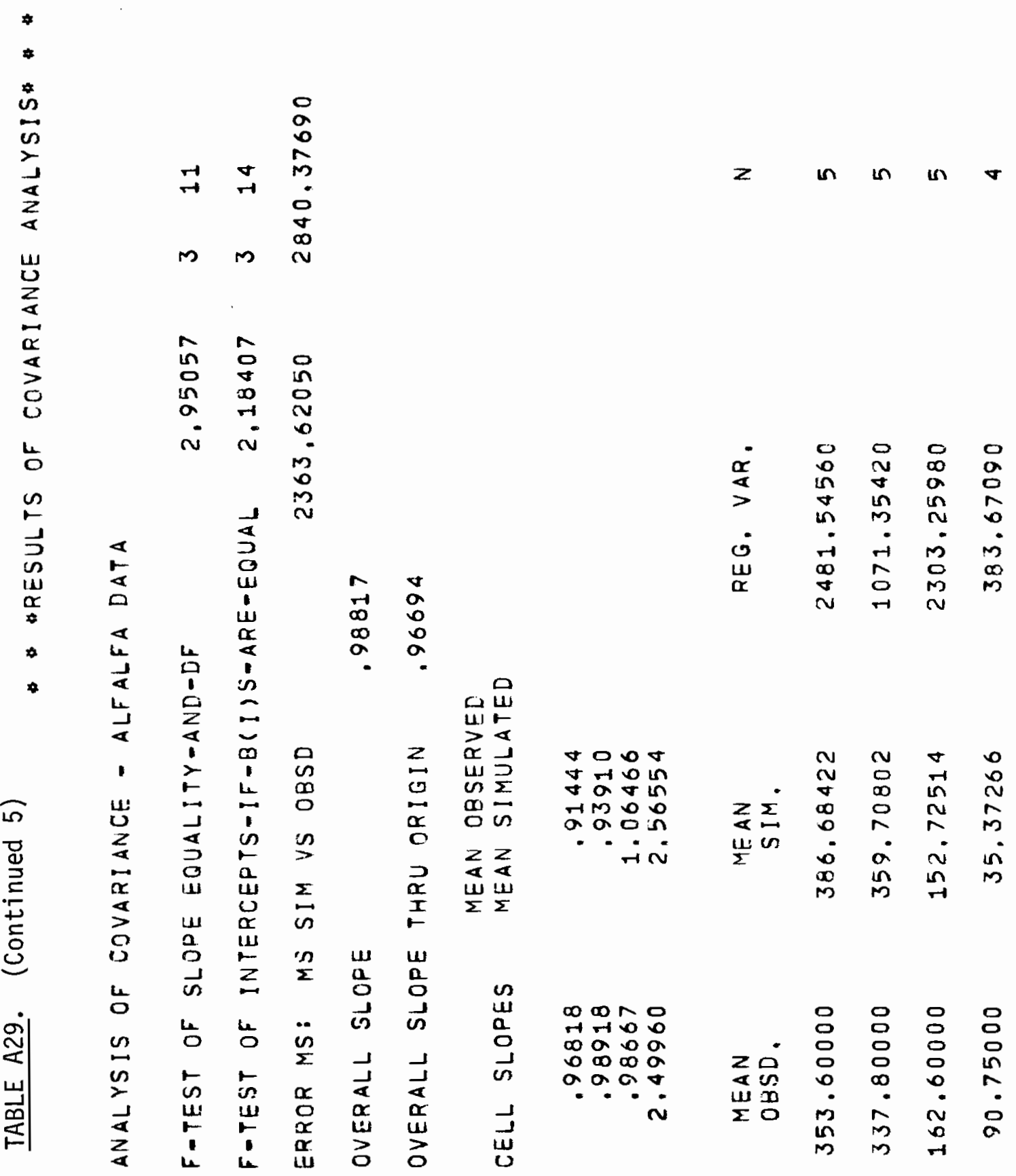



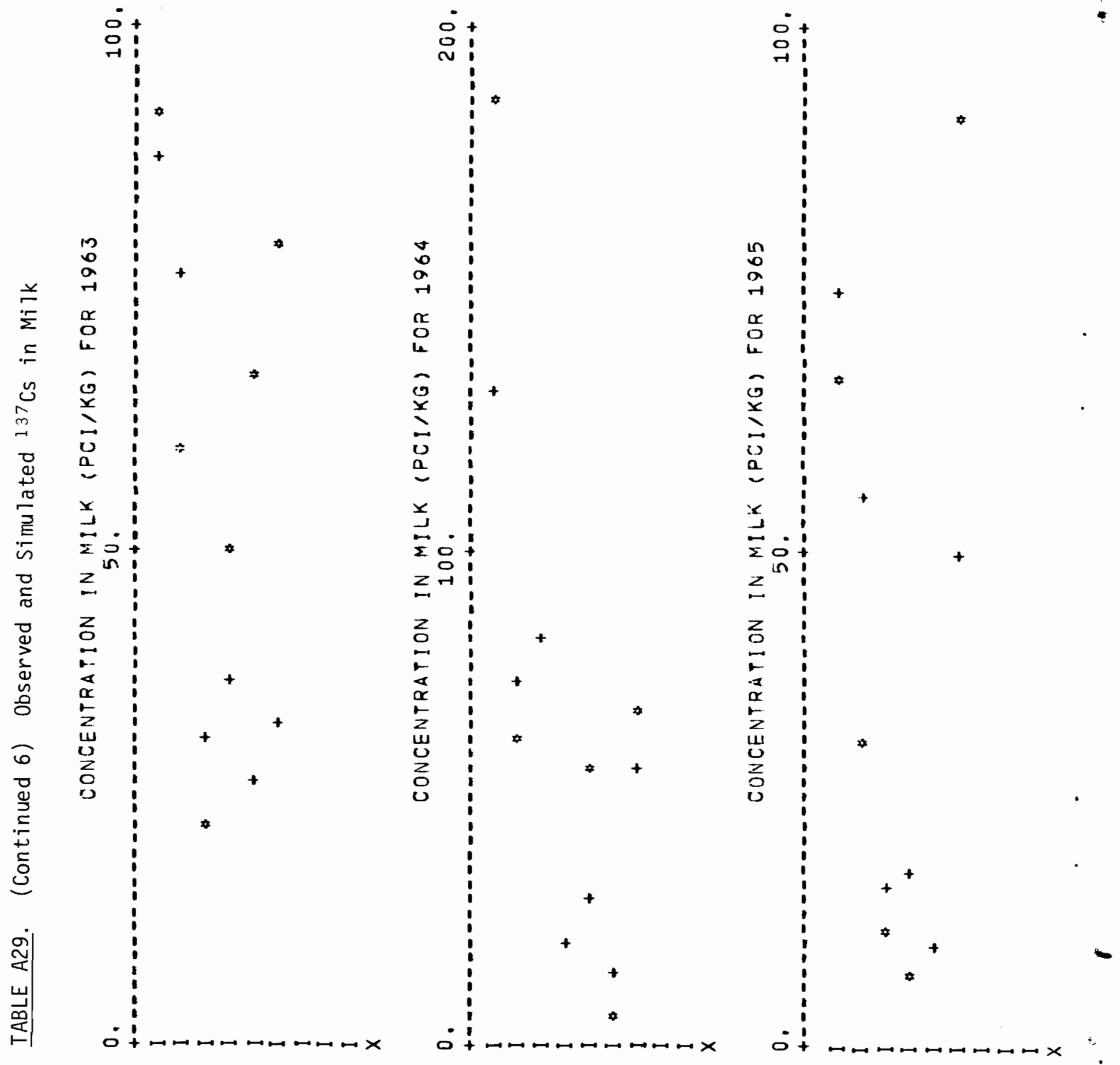
0 in $\forall 0$ in $0 \infty+0 \infty$ in a $\infty 0 \infty \infty$ - imini in $\rightarrow$ in $\vec{\nabla}$ o d 0 mon - in $\infty N M M N$

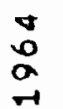

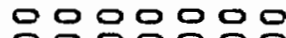

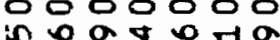
onmomio manm tin a m $\rightarrow$ a

$n$
0
0

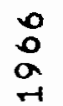

000000 000000 romino.

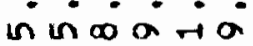
$\sin \ln ^{4}$ $\circ 0$ 응. $\rightarrow-$ mo 


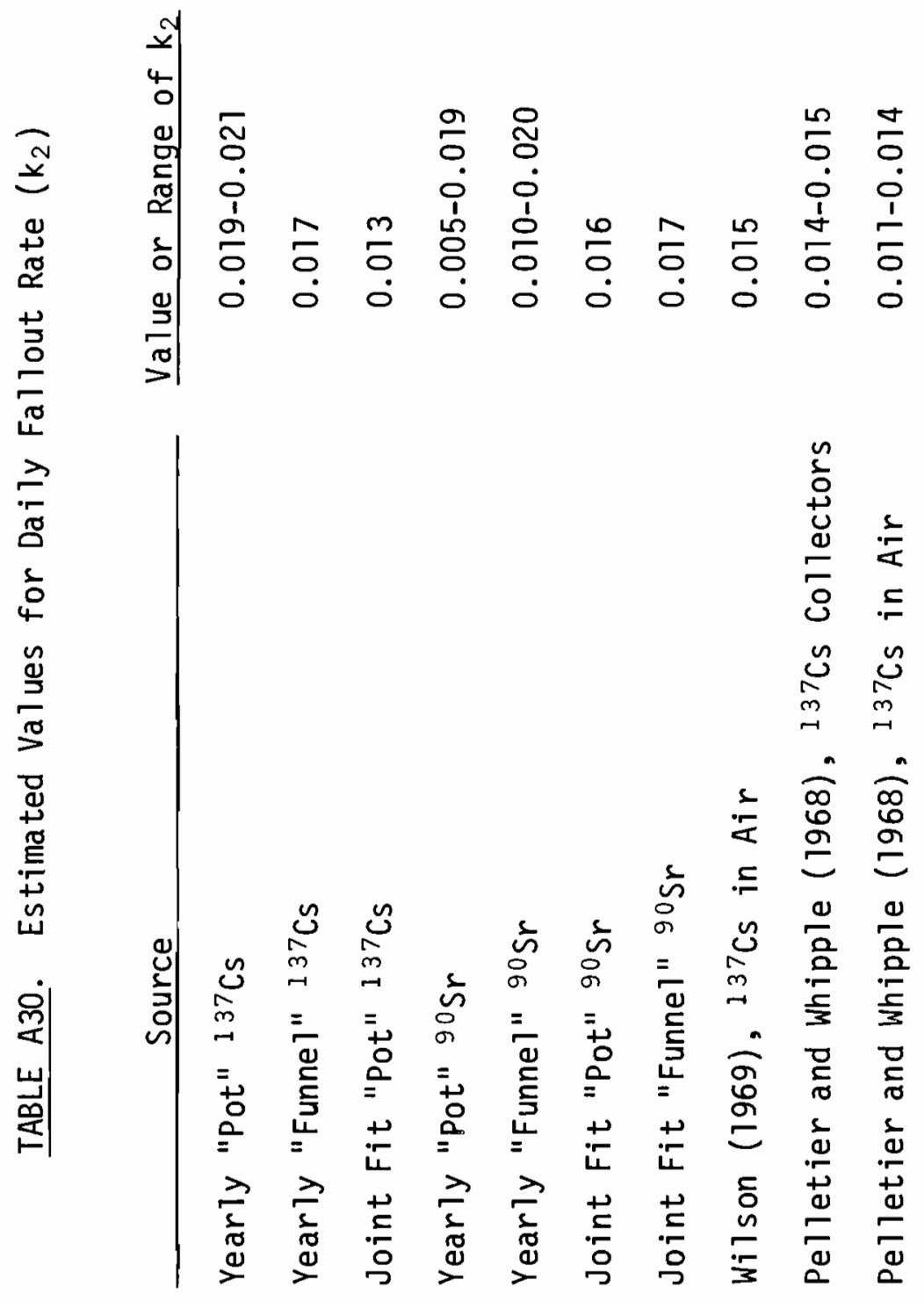


TABLE A31. Calculated Daily Fallout Values for ${ }^{137} \mathrm{Cs}$ and ${ }^{90} \mathrm{Sr}$ $\left(\mathrm{pC} \mathrm{i} / \mathrm{m}^{2}\right)$ at Saint George, Utah

\begin{tabular}{|c|c|c|c|c|}
\hline & Year & Day & ${ }^{137} \mathrm{Cs}$ & $90 \mathrm{Sr}$ \\
\hline 100 & 1 & 1 & 19,0448 & 24.17530 \\
\hline 111 & 1 & 2 & 19,2747 & 24.46721 \\
\hline 120 & 1 & 3 & 18.5074 & 24.16259 \\
\hline 130 & 1 & 4 & 19.7429 & 25.06153 \\
\hline $14 i$ & 1 & 5 & 19.9813 & 25.36408 \\
\hline 156 & 1 & 6 & 20.2225 & 25.071120 \\
\hline 164 & 1 & 7 & 20.4600 & 25,98018 \\
\hline $17 n$ & 1 & $d$ & 20.7137 & 26.29532 \\
\hline 180 & 1 & 7 & 20.9635 & 20.61125 \\
\hline 190 & 1 & 10 & 21.2109 & 26.93251 \\
\hline 200 & 1 & 11 & 21.4730 & 27.25764 \\
\hline 210 & 1 & 12 & 21.7322 & 27.50070 \\
\hline $221 j$ & 1 & 13 & 21.9945 & 27.91374 \\
\hline $23 ! 5$ & 1. & 14 & 22.2001 & 20.25600 \\
\hline 240 & 1 & 15 & 22.5283 & 28.59792 \\
\hline 250 & 1 & 16 & 22.8009 & 28.94316 \\
\hline 200 & 1 & 17 & 23.0701 & 29.29257 \\
\hline $27 i$ & 2 & $1 \xi$ & 2.3 .3540 & 20,64620 \\
\hline 280 & 1 & 19 & 23.0360 & 30.96410 \\
\hline 240 & 1 & 20 & 23.7210 & 30.35032 \\
\hline 300 & 1 & 21 & $24.21 \cup 7$ & 30.732 .91 \\
\hline 310 & 1 & 22 & 24.5030 & 31.10592 \\
\hline 320 & 1 & 23 & 24.7980 & $\$ 1.47 \% 42$ \\
\hline 331 & 1 & 24 & 25.0902 & 31,03945 \\
\hline 340 & 1 & 22 & 25.4012 & 52.24406 \\
\hline 350 & 1 & 26 & $25.707 \mathrm{j}$ & 32.03332 \\
\hline 300 & $\therefore$ & 27 & 20.01 .82 & 33.02728 \\
\hline 370 & 1. & 28 & 26.3323 & 33.42600 \\
\hline ن & 2 & 23 & 26.6502 & 33.22953 \\
\hline 390 & 1 & 30 & 20.9719 & 34.23793 \\
\hline $40 u$ & 1 & 31 & 27.2975 & 34.55126 \\
\hline 416 & 1 & $3 ?$ & 27.0271 & 3 . 30958 \\
\hline 420 & 1 & 33 & 27.9606 & 35,49295 \\
\hline 430 & 1 & 34 & 23.2931 & 35,32143 \\
\hline 440 & 1 & 35 & 28.0398 & 50,35508 \\
\hline 450 & 1 & 36 & 20.9355 & 36.79397 \\
\hline 460 & 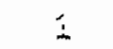 & 37 & 29.3554 & 37.23016 \\
\hline 470 & $i$ & 33 & 29.6996 & .57 .50771 \\
\hline 480 & 1 & 39 & 30.0480 & 51,14268 \\
\hline 490 & 1 & 40 & $31) .4107$ & 38.60315 \\
\hline 500 & 1 & 41 & 30.7779 & 39,06910 \\
\hline 510 & 1. & 42 & 31.1494 & 39.54034 \\
\hline 520 & 1 & 43 & 31.52 .55 & 40.01819 \\
\hline 530 & 1 & 44 & 31,9061 & 40,50130 \\
\hline 540 & 1 & 45 & 32.291 .2 & 40.99024 \\
\hline 550 & 1 & 46 & 32,6311 & 41.48509 \\
\hline 501 & 1 & 47 & 33.0750 & 41.90591 \\
\hline 573 & 1 & 48 & 3.3 .4748 & 42.49277 \\
\hline 280 & 1 & 49 & 33.8790 & 43.00575 \\
\hline 5 & 1 & 50 & 34.2090 & $43,524 y 3$ \\
\hline 600 & 1 & 51 & 34.7020 & $44.05 \cup 38$ \\
\hline 010 & 1 & 52 & 35.1209 & 44,53217 \\
\hline
\end{tabular}


TABLE A31. (Continued 2)

\begin{tabular}{|c|c|c|c|c|}
\hline 020 & 1 & 53 & 35.5449 & 45.12038 \\
\hline 630 & $\overline{1}$ & 54 & 35.9740 & 43.60508 \\
\hline 040 & $\overline{1}$ & 55 & 36.4000 & 45,21030 \\
\hline 050 & 1 & 56 & 35.0478 & 40,77430 \\
\hline 600 & 1 & 57 & 37.2920 & 47.33893 \\
\hline 070 & 1 & 58 & 37.7420 & $47.91 \cup 76$ \\
\hline 600 & 1 & 59 & $3,9,1935$ & 43.43885 \\
\hline $691 \mathrm{~J}$ & 1 & 50 & 30.01590 & 47.07423 \\
\hline 700 & 1 & 61 & 39.1263 & 48.60600 \\
\hline 710 & 1 & $5 ?$ & 39.5797 & ju. 20025 \\
\hline 720 & 1 & 63 & 40.0767 & 50.87308 \\
\hline 750 & 1 & 64 & 40.5000 & 51,48724 \\
\hline 740 & 1 & jo & 41.0502 & 32.10381 \\
\hline 750 & 1 & 60 & 41.5436 & 52,73780 \\
\hline 760 & 1 & 67 & 42.4473 & 53.37455 \\
\hline 770 & 1 & 00 & 42.5549 & 54.01890 \\
\hline 780 & 1 & 69 & 4.5 .0687 & 54.67103 \\
\hline 790 & 1 & 70 & 43.5830 & 25.33104 \\
\hline 800 & 1 & 71 & 44.1143 & 53.99901 \\
\hline 810 & $i$ & 12 & 44.0474 & 20.67504 \\
\hline 320 & 1 & 73 & 45.1804 & $57.5,5924$ \\
\hline 83. & $i$ & 74 & 40.7319 & 20.05170 \\
\hline 840 & 1 & 75 & 40.2840 & 58.75252 \\
\hline 850 & $\overrightarrow{1}$ & 76 & 40.8427 & 59.46179 \\
\hline 860 & 1 & 77 & 47.4032 & 00.17963 \\
\hline 870 & 1 & $7 \ddot{3}$ & 47.9806 & 50, צఏo 14 \\
\hline 884 & $i$ & 79 & 48.5598 & 61.64142 \\
\hline 090 & 1 & 83 & 49.1700 & 62.30557 \\
\hline 900 & 1 & 81 & 49.7393 & 53.13870 \\
\hline 910 & 1 & 82 & 50.3590 & 03.90093 \\
\hline 920 & 1 & 63 & 50.9475 & 64.67236 \\
\hline $93 i$ & $\hat{1}$ & 84 & 51.5620 & 65.45311 \\
\hline $94 \mathrm{U}$ & 1 & 35 & 52.1820 & 60.24328 \\
\hline 950 & 1 & 86 & 52.3150 & $07.114<9$ \\
\hline $90 \mathrm{U}$ & 1 & 87 & 53.4526 & 67.652 .3 \\
\hline 970 & 1 & 88 & 54.0979 & 68,157148 \\
\hline 984 & 1 & 89 & 54,7510 & 09.50050 \\
\hline $9 y$ & 1 & 90 & 55.4120 & 70.33953 \\
\hline 1000 & $\overline{1}$ & 91 & 56.0804 & 71.13059 \\
\hline 1010 & 1 & 92 & 56.7530 & 12.04810 \\
\hline 1020 & 1 & 93 & 61.4732 & 22.87548 \\
\hline 1030 & 1 & 94 & 02.2203 & 25.1516 \\
\hline 1040 & $\overrightarrow{1}$ & 95 & 62.9715 & 23,43113 \\
\hline 1050 & 1 & 96 & $63,7.317$ & 23.71400 \\
\hline 1060 & 1 & 97 & 04.5011 & 24,03028 \\
\hline $107 \mathrm{~J}$ & 1 & 96 & 65.2738 & 24.29002 \\
\hline 1080 & $\overline{1}$ & 99 & 06.0078 & 24,20326 \\
\hline 1090 & 1 & 100 & 60.9654 & 24,88003 \\
\hline $11 \cup !$ & 1 & 101 & 67.0726 & 25.18037 \\
\hline 11.10 & 1 & 102 & $68.40 \% 6$ & 25.48438 \\
\hline 1120 & 1 & 103 & 69.3104 & 25.74205 \\
\hline 1130 & $i$ & 104 & $70.1 .53 ?$ & $26.1 \cup 340$ \\
\hline 1140 & 1 & 105 & 71.0001 & $\$ 0.41853$ \\
\hline 1150 & 1 & 106 & 71.857 .5 & 20.7374 \\
\hline
\end{tabular}


TABLE A31. (Continued 3)

\begin{tabular}{|c|c|c|c|c|}
\hline 1160 & 1 & 107 & 72.7243 & 27.00025 \\
\hline $11 \%$ & 1 & 108 & 73.0027 & 27.38092 \\
\hline 1100 & 1 & 103 & 74.4913 & 27.71755 \\
\hline 1194 & 1 & 110 & 75.3906 & 28.0521 \\
\hline 1200 & 1 & 111 & 76.3007 & 20.3998 \\
\hline 1210 & 1 & 112 & $77,221.8$ & 20.7335 \\
\hline $122 \mathrm{u}$ & 1 & 113 & 78.1541 & 23.2452 \\
\hline 1230 & 1 & 114 & 79.0970 & 27.1750 \\
\hline 1240 & 1 & 115 & 80.0524 & 27.3048 \\
\hline 1254 & $\Sigma$ & 110 & $8: .01 .09$ & 26,3446 \\
\hline 1260 & 1 & 117 & 81.9969 & 26.3921 \\
\hline 1270 & 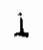 & 118 & 62.9800 & 25.9472 \\
\hline 1281$)$ & 1 & 117 & $83.98 \mathrm{~m} 7$ & 25,5090 \\
\hline 1290 & 1 & 120 & 85.0020 & 25,0798 \\
\hline 1300 & 1 & 121 & 80.0200 & 24,0571 \\
\hline 1310 & 1 & 122 & 87.0073 & 24,2414 \\
\hline 1320 & 1 & 123 & 80.1184 & 23.8328 \\
\hline $133 \mathrm{j}$ & 1 & 124 & 89.1822 & 2.5 .4311 \\
\hline 1340 & 1 & 125 & 90,2507 & 23.03617 \\
\hline 1350 & 1 & 126 & 91.3485 & 22.54787 \\
\hline 1300 & 1 & 127 & 92.4513 & 22.2661 \\
\hline 1370 & 1 & 123 & 93.5074 & 21,6907 \\
\hline 1380 & 1 & $12 ?$ & 94,0970 & 21.5217 \\
\hline 1390 & 1 & 130 & 95.8412 & 21,1590 \\
\hline 1400 & 1 & 131 & 36,5972 & 20.5023 \\
\hline 1410 & 1 & 1.32 & 98.1002 & $20,4 b 169$ \\
\hline $142 \mathrm{~J}$ & 1 & 133 & 99.3533 & 20,10695 \\
\hline 1430 & 1 & 134 & $100.552 \%$ & 14.15002 \\
\hline 1440 & $i$ & 135 & 98.8577 & 19,4340 \\
\hline 1450 & 1 & 130 & 97.1914 & 19.1072 \\
\hline 1400 & $i$ & 1.37 & 95.5531 & 16.785 \\
\hline 1470 & 1 & 130 & 9.3 .9424 & 15,468 \\
\hline 1480 & 1 & 139 & 92.3509 & 10,15717 \\
\hline $149 \mathrm{U}$ & 1 & 14. & 90.3021 & .77 .85111 \\
\hline 1500 & 1 & 141 & 89.2715 & 17.5502 \\
\hline 1510 & 1 & 142 & 31.1953 & 55,2860 \\
\hline 1520 & 1 & 145 & 79.8200 & 34,354 \\
\hline 1530 & 1 & 144 & 78.4811 & 53,430 \\
\hline 1540 & 1 & 145 & 77.1532 & 52.537 \\
\hline $155 \mathrm{~J}$ & 1 & 140 & 75.3576 & 51.65175 \\
\hline 1560 & 1 & 147 & 74.5789 & 50.78110 \\
\hline 1570 & 1 & 143 & 73.3210 & 49.9251 \\
\hline 1580 & 1 & 149 & 72.0858 & 49.083 \\
\hline 1590 & 1 & 151 & 70.8707 & 48.250 \\
\hline 1600 & 1 & 151 & 69.0761 & 47.4427 \\
\hline 1010 & 1 & 152 & 63,5010 & 40.0431 \\
\hline $102 \mathrm{~J}$ & 1 & 153 & 67.3460 & 45.856 \\
\hline 1630 & 1 & 154 & 66.2117 & 45.063 \\
\hline 1640 & 1 & 155 & 65.1950 & 44.32390 \\
\hline 1050 & 1 & 156 & 63.9984 & 43,57677 \\
\hline 1660 & 1 & 157 & 02.9190 & 42,34222 \\
\hline $167 \%$ & 1 & 153 & $61.859 \mathrm{~J}$ & 42.12006 \\
\hline 1680 & 1 & 159 & 60.3160 & 41.41007 \\
\hline 1090 & 1 & 160 & 59.7911 & 40.7120 \\
\hline
\end{tabular}

\section{A. 141}


TABLE A31. (Continued 4)

\begin{tabular}{|c|c|c|c|c|}
\hline 1700 & 1 & 161 & 50.7835 & 40.02580 \\
\hline 1710 & 1 & 162 & 57.7924 & 30.35111 \\
\hline 1720 & 1 & 16.3 & 50.8182 & 38.60779 \\
\hline 1730 & 1 & 164 & 51.9645 & 30.03060 \\
\hline 1740 & 2 & 205 & 54.9109 & 37.3945 \\
\hline 1750 & 1 & 160 & 53.9932 & 36,7641 \\
\hline 1760 & 1 & 167 & 53.0530 & $56,1.444$ \\
\hline 1770 & 1 & 160 & $5 ?, 1802$ & 35.5352 \\
\hline 1780 & 1 & 169 & 51.3002 & 34.9562 \\
\hline 1790 & 1 & $17 !$ & 50.4437 & 34.3473 \\
\hline 1800 & 1 & 171 & $4 ? .5934$ & 33.7083 \\
\hline $18 \leq 1 i$ & 1 & 172 & 46.7574 & 33,1991 \\
\hline 1020 & 1 & 173 & 20.272 .4 & 25.0945 \\
\hline $103 i$ & 1 & 174 & 27.7950 & 14.134507 \\
\hline 1840 & 1 & 175 & $2 \% .3273$ & 14.56393 \\
\hline 1556 & 1 & 176 & 20.8501 & 24.34399 \\
\hline $180 \mathrm{~J}$ & 1 & 177 & 20.4130 & 14.10221 \\
\hline 1070 & 1 & 178 & 25.9000 & 13.86450 \\
\hline 1080 & 1 & 179 & 25.5300 & 13.65079 \\
\hline 1690 & 1 & 180 & 25.1045 & 13.46103 \\
\hline 1900 & 1 & 181 & 24.6774 & 1.5 .17513 \\
\hline 1911 & 1 & 132 & 24.2514 & 12.75305 \\
\hline 1920 & 1 & 183 & 23.8525 & 12.13471 \\
\hline $1 y 30$ & 1 & 184 & 23.4504 & 12.52005 \\
\hline 1940 & 1 & 135 & 25.11551 & 12.30901 \\
\hline 1950 & 1 & 130 & 22.0665 & 12.10152 \\
\hline $196 !$ & 1 & 197 & 22.2844 & 11.89753 \\
\hline 1970 & 1 & 183 & 21.9030 & 11.64699 \\
\hline 1980 & 1 & $18 ?$ & $21.53+5$ & 11.49782 \\
\hline 1940 & 1 & 190 & 21,1704 & $11,3 \cup 597$ \\
\hline 2000 & 1 & 191 & 211,3174 & 11.11540 \\
\hline 2010 & 1 & 192 & 20.4605 & 10.92003 \\
\hline 2020 & 1 & 1.93 & 211.1235 & 14.74683 \\
\hline 20315 & 1 & 194 & 10.7043 & 10.56372 \\
\hline 2040 & 1 & 1.95 & $19,45 \cup 3$ & 10.38468 \\
\hline 2050 & 1 & 190 & 19,1229 & 10.24963 \\
\hline 2060 & 1 & 197 & 10.8006 & 10,03753 \\
\hline 2070 & 1 & 198 & 10.4037 & 9.86034 \\
\hline 208.0 & 1 & $19^{\circ}$ & 18.1721 & $9.7 \cup 193$ \\
\hline 2090 & 1 & 200 & 17.8055 & 9.53045 \\
\hline 2101 & 1 & 201 & 17.5046 & 9.37767 \\
\hline 2110 & 1 & 202 & 17.2606 & $\exists .21960$ \\
\hline $212 j$ & 1 & 203 & 16.9770 & 9.00419 \\
\hline 2130 & 1 & 204 & 10.0915 & 8.91140 \\
\hline 2140 & 1 & 205 & 10.4099 & 3.76119 \\
\hline 2150 & 1 & 206 & 10.1333 & $8.01 .351]$ \\
\hline 2160 & 1 & 207 & 15.8014 & 8.46831 \\
\hline 2170 & 1 & 2018 & 15.5944 & 28.95910 \\
\hline 2160 & 1 & 200 & 15.3312 & 28.47096 \\
\hline 2140 & 1 & 210 & 15,0727 & 27.39104 \\
\hline 2200 & 1 & 211 & 14.8137 & 27.51922 \\
\hline 22111 & 1 & 212 & 14.5089 & 21.05234 \\
\hline$<220$ & 1 & 213 & 14.3233 & 26.59927 \\
\hline 2231 & 1 & 214 & 52,0095 & 26.120 \\
\hline
\end{tabular}


TABLE A31. (Continued 5)

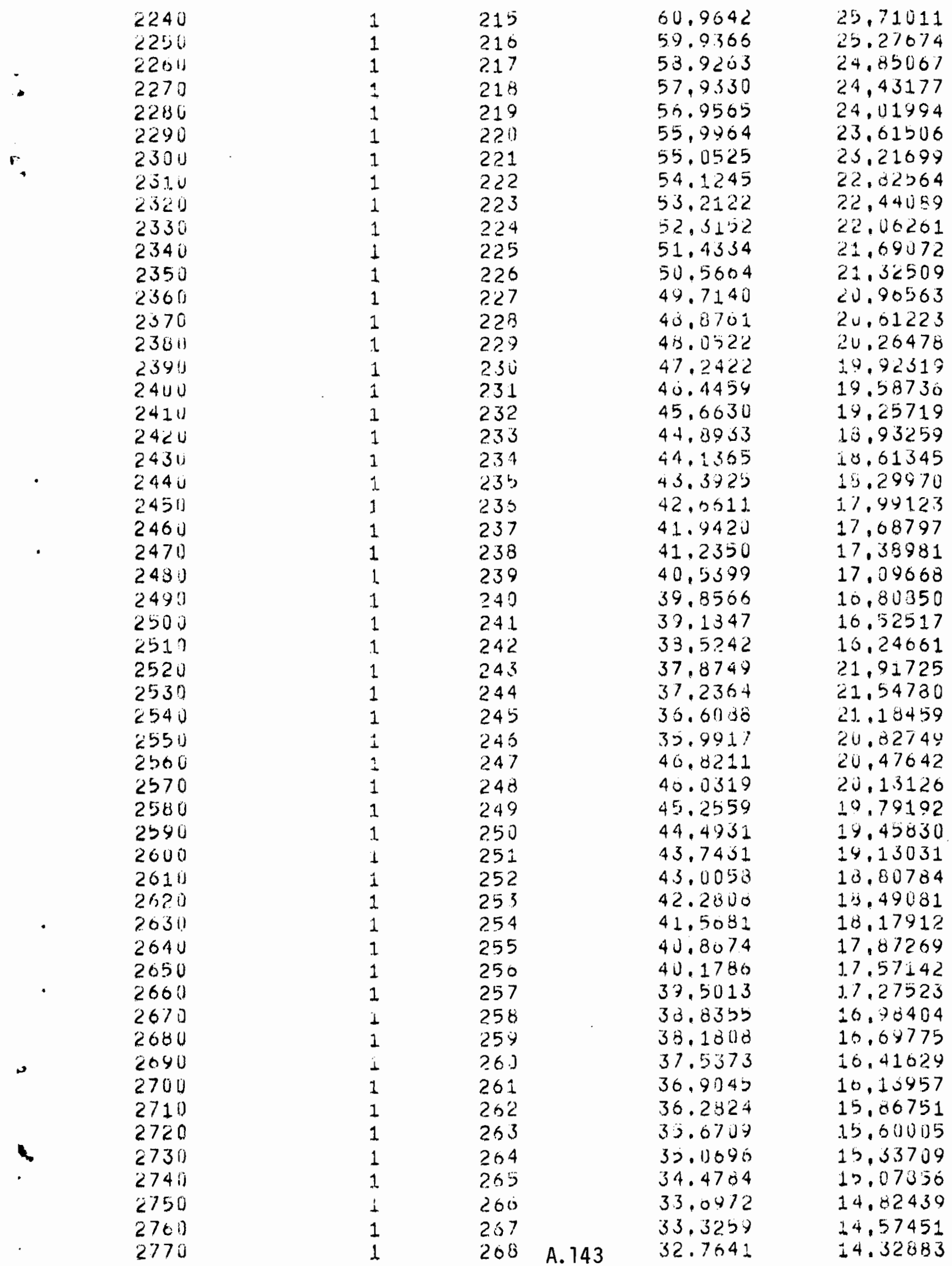


TABLE A31. (Continued 6)

\begin{tabular}{|c|c|c|c|c|}
\hline$<760$ & 1 & 2.69 & 32,2113 & 14.08730 \\
\hline 2790 & $i$ & 270 & 31.6007 & 13,64984 \\
\hline 2000 & j & 271 & 31.1350 & 13,51638 \\
\hline 2810 & 1 & 272 & 30,0192 & 13.50686 \\
\hline 2820 & 1 & 273 & 30.0942 & 13,1012 \\
\hline 2830 & 1 & 274 & 23.5870 & 12.9393 \\
\hline 2840 & $i$ & 272 & 24.0383 & 12,7212 \\
\hline 2850 & 1 & 270 & 28,5019 & $1<, 5000$ \\
\hline 2450 & $i$ & 217 & 28.1159 & 12.2950 \\
\hline 2870 & 1 & 275 & 27.6419 & 12.08037 \\
\hline 2880 & 1 & 279 & $27.176 \mathrm{~J}$ & 11.3845 \\
\hline 2690 & 1 & $28 u$ & 26,7177 & $\$ 1.6040$ \\
\hline$<900$ & 1 & 231 & 20.2675 & 11.4076 \\
\hline 2910 & 1. & 2.82 & 25.3240 & $\$ 1.2940$ \\
\hline$\angle 920$ & 1 & 283 & 11.0597 & 5.0784 \\
\hline 2930 & 1 & 234 & $1 \pm .00155$ & 3,7228 \\
\hline$<y+0$ & 1 & 285 & 11.9430 & .3 .7677 \\
\hline 2450 & 1 & 230 & 12.0871 & 3.8132 \\
\hline 2060 & 1. & $28 \%$ & 12.2331 & 3.8592 \\
\hline 2970 & 1. & 283 & 12.3807 & 3,9058 \\
\hline 2980 & \pm & 289 & 1?.5302 & 3.1530 \\
\hline 2990 & 1 & 2911 & 32.6815 & 4.0007 \\
\hline 3000 & 1 & 291 & 12.8346 & 4.0490 \\
\hline 3010 & 1 & 292 & 13.9895 & 4.0979 \\
\hline 3020 & 1 & 293 & 13,1463 & 4.1473 \\
\hline 30.30 & 1 & 291 & 13.3050 & 4.1974 \\
\hline 3040 & 1 & 295 & 13.4657 & 4.2481 \\
\hline 3050 & 1 & 290 & 13.6292 & 4.2944 \\
\hline 3060 & $i$ & 297 & 13.7927 & 4.3513 \\
\hline 3070 & 1 & 230 & 13.9592 & 4,4038 \\
\hline 3080 & 1 & 277 & 14.1278 & 4.4570 \\
\hline 3090 & 1 & 300 & 14.2783 & $4,51 \cup 8$ \\
\hline 3100 & 1 & 301 & $14,47 \mathrm{JU}$ & 4.5652 \\
\hline 3110 & 1 & 302 & 14.0450 & 4.0204 \\
\hline 3120 & 1 & 303 & 14.3224 & 4.6761 \\
\hline 3130 & $i$ & 31) 4 & 15.11014 & 4.7326 \\
\hline 3140 & 1 & 305 & 10.1820 & 4.1897 \\
\hline 3150 & $i$ & 306 & 15.3050 & 4.8475 \\
\hline 3100 & 1 & 307 & 15.5510 & 4.9001 \\
\hline 3170 & 1 & $30 \mathrm{~s}$ & 15.7390 & 4.9053 \\
\hline 3180 & 1 & 309 & 15.9290 & 5.0252 \\
\hline 3190 & 1 & 310 & 16.1213 & 5.085 \\
\hline 3200 & 1 & 311 & 16.3159 & 5.1473 \\
\hline $321 \mathrm{u}$ & 1 & 3.12 & 16.5129 & 5.2094 \\
\hline 3220 & 1 & 313 & 16,7123 & 5.2723 \\
\hline 3230 & \pm & 314 & 16.9140 & 5.3300 \\
\hline 3240 & 1 & 315 & 17.1182 & 2.40044 \\
\hline 3250 & 1 & 316 & 17.3249 & 3.4056 \\
\hline 3260 & 1 & $31 \%$ & 17.3370 & 5,53162 \\
\hline 3270 & 1 & 318 & $1 / .7457$ & 5.59840 \\
\hline 3280 & 1 & 319 & 17.9579 & 5.505 \\
\hline 32.90 & 2 & 320 & 13.1767 & 3.75438 \\
\hline 3300 & 1 & 321 & 15.3902 & 2,5036 \\
\hline 3310 & 1 & 322 & 13.018 .5 & 5.873 \\
\hline
\end{tabular}


TABLE A31. (Continued 7)

\begin{tabular}{|c|c|c|c|c|}
\hline 3320 & t & $3 ? 3$ & 18.3430 & 5,94458 \\
\hline 3330 & 1 & 324 & 17.0705 & 0.01635 \\
\hline 3340 & 1 & 325 & 19.3007 & 6,46898 \\
\hline 3350 & 1 & 320 & 19,2337 & 6.16279 \\
\hline 3360 & $i$ & 327 & 13.7695 & 6.23638 \\
\hline 3370 & 1 & 328 & 20.10082 & 6.31217 \\
\hline 3380 & 1 & 329 & 20.2497 & 6.38338 \\
\hline 3390 & 1 & 330 & 20.4942 & 0.45550 \\
\hline 3400 & 1 & 331 & 20.7416 & 6.24355 \\
\hline 3410 & 1 & 332 & 20.9920 & 6.62 .25 \\
\hline 3420 & 1 & 3.33 & $21,245.4$ & 0.70251 \\
\hline 3430 & 1 & 334 & 21.5019 & 6.78341 \\
\hline 3440 & $i$ & 335 & 21.7615 & 0.30530 \\
\hline 5450 & 1 & 336 & 22.9242 & 0.9431 \\
\hline 3400 & 1 & 337 & 22.2901 & 1.0320 \\
\hline 3470 & 1 & 330 & 22.5572 & 7.1109 \\
\hline 3480 & 1 & 339 & 22,3315 & 7.20283 \\
\hline 3490 & 1 & 340 & 23.1072 & 7.2898 \\
\hline 3500 & 1 & 341 & 23,3861 & 7.37784 \\
\hline 3510 & 1 & 342 & 23.5634 & 7.40690 \\
\hline 3520 & 1 & 343 & 23,9542 & 7.55705 \\
\hline 3530 & 1 & 344 & 24.2435 & 7.84023 \\
\hline 3540 & 1 & 345 & 24.5360 & 7.74061 \\
\hline 3550 & 1 & 346 & 24.8322 & 7.8340 \\
\hline 3560 & 1 & 347 & 25.1320 & 7,92863 \\
\hline 3570 & 1 & 343 & 25.4354 & 3.02435 \\
\hline 3580 & 1 & 3.73 & 25,7425 & $8.1<122$ \\
\hline 3590 & 1 & 350 & 26.0532 & 6.21926 \\
\hline 3600 & 1. & 351 & 26.3678 & 0,51849 \\
\hline 3010 & 1 & 352 & 26.6361 & 8,41831 \\
\hline 3021 & 1 & 353 & 27.0032 & 3.52025 \\
\hline 3630 & 1 & 354 & 27.3343 & 0.62341 \\
\hline 3641 & 1 & 355 & 27.0043 & 0.72751 \\
\hline 3650 & 1 & 356 & 27.9983 & 8.83257 \\
\hline 3600 & 1 & 357 & 28.3363 & 8,93951 \\
\hline $367 \mathrm{U}$ & 1 & 358 & 28,6783 & 9.04743 \\
\hline 3680 & 1 & 359 & 29.0240 & 9.15665 \\
\hline 3690 & 1 & 360 & 29.3750 & 9.20719 \\
\hline 3700 & 1 & 361 & 29.7290 & 9.379017 \\
\hline 3710 & 1 & 352 & 39.0805 & 9,49229 \\
\hline 3720 & 1 & 365 & 30.4517 & 9.0068 \\
\hline 3750 & $\perp$ & 364 & 30.8193 & 9,12280 \\
\hline 3740 & 1 & 355 & 31.1414 & 9.84024 \\
\hline 3750 & $?$ & 1 & 31.5679 & $7.954 \mathrm{~J} 4$ \\
\hline 3760 & 2 & 2 & $31.9491 j$ & 10.07926 \\
\hline 3770 & 2 & 3 & 32.3347 & .10 .20095 \\
\hline 3780 & 2 & 4 & 32,7251 & 10.32409 \\
\hline 3790 & $?$ & 5 & 33.3 .202 & 10,44873 \\
\hline 3600 & 2 & 0 & 35.5201 & 10.57407 \\
\hline 3810 & 2 & 7 & 33.9247 & $10.7 \cup 253$ \\
\hline 3820 & 2 & 3 & 13.0071 & $0,3666 ?$ \\
\hline 3830 & 2 & 3 & 10.8310 & 5.87300 \\
\hline 3845 & 2 & 19 & 19.0591 & $\forall .108199$ \\
\hline 3850 & 2 & 11 & 19.2892 & 4.19103 \\
\hline
\end{tabular}


TABLE A31. (Continued 8)

\begin{tabular}{|c|c|c|c|c|}
\hline $386 \mathrm{v}$ & $?$ & 12 & $14.52<0$ & 4.30259 \\
\hline 3870 & 2 & 13 & 13.7577 & $\because .41490$ \\
\hline $580 \mathrm{~J}$ & 2 & 14 & 19.9762 & 4.52856 \\
\hline J 359 & $?$ & 15 & 20.2370 & 9.64359 \\
\hline 3900 & 2 & 10 & 20.4820 & 9.76001 \\
\hline $391 \mathrm{~J}$ & $?$ & 17 & 20.7292 & 9.07783 \\
\hline 3923 & 2 & 13 & 20.9795 & 9.99700 \\
\hline 3930 & 2 & 10 & 21.2027 & 10.11777 \\
\hline 3941 & $?$ & 21 & 21.4831 & $16.23 \times 31$ \\
\hline $595 i$ & ? & 21 & 21.7 .432 & 16,36553 \\
\hline 3900 & 2 & 22 & 22.0110 & 10.40857 \\
\hline 3970 & $?$ & 23 & $2 ? .2700$ & 10.01227 \\
\hline $396 \mathrm{~J}$ & 2 & 24 & 22.5437 & 10.74542 \\
\hline 3990 & 2 & 27 & 22.8179 & 10.87321 \\
\hline $400 \mathrm{~J}$ & 2 & 20 & 23,11453 & 11,0438 \\
\hline 4010 & $?$ & 27 & 23.3721 & 12.1372 .3 \\
\hline 4023 & ? & 23 & 23.0543 & 12.27163 \\
\hline 4030 & 2 & 29 & 23.9593 & 12.40775 \\
\hline 4041 & ? & $3 i j$ & 24,2239 & 11.54547 \\
\hline 405.1 & 2 & $3 i$ & $24,5 ? 14$ & 11.68485 \\
\hline 41060 & 2 & 32 & $24.8,74$ & 11.02591 \\
\hline 4070 & 2 & 35 & 25,1170 & 11.96500 \\
\hline 40811 & 2 & 34 & 23.4202 & 12.11317 \\
\hline 4096 & 2 & 35 & 25.7271 & 12.25940 \\
\hline 4100 & $?$ & 35 & 20.0377 & 12.40741 \\
\hline $411 ! j$ & 2 & 37 & 22.3520 & 12.2571 \\
\hline 4120 & 2 & 3.3 & 20.0701 & 12,714078 \\
\hline 41313 & 2 & $3 \cdot)$ & 20.9721 & \pm 2.56221 \\
\hline 4140 & 2 & $4 !$ & 27,3100 & 13.01748 \\
\hline 4150 & 2 & 41 & 27.5477 & 13.17463 \\
\hline 4160 & 2 & $4 \%$ & 21.7812 & .13 .33368 \\
\hline $4 i 7 \mathrm{iJ}$ & $?$ & 43 & 23,3193 & 13,49405 \\
\hline 4.180 & 2 & 44 & 23.6612 & 15.0575 \\
\hline 4190 & 2 & 45 & 29.0072 & 13.82244 \\
\hline 4200 & ? & 40 & 29.3574 & 13.70931 \\
\hline 4210 & $?$ & 47 & 27.7118 & 14.15019 \\
\hline 4220 & 2 & 48 & 30.070 & 14.32911 \\
\hline 4230 & 2 & 49 & 30.4333 & 24.5021 \\
\hline $424 !$ & 2 & 50 & 30.8009 & $1+.6771$ \\
\hline 42.53 & $?$ & 51 & 31.1720 & 14.82436 \\
\hline 4260 & 2 & 52 & $31,54 y 1$ & 15,3368 \\
\hline $427 \mathrm{~s}$ & 2 & 53 & 31.9299 & $1 b, 21 b 1$ \\
\hline 4280 & 2 & 54 & 32.3124 & 12,3968 \\
\hline 4290 & $?$ & 53 & 32.705 & 15,70475 \\
\hline $430 \mathrm{iJ}$ & 2 & 5o & 33.1004 & 12.7729 \\
\hline 43111 & 2 & 57 & 35.5000 & 15.90331 \\
\hline 4320 & 2 & 58 & 33.9044 & 10,15603 \\
\hline 4.330 & 2 & 59 & 34.3157 & 10.35107 \\
\hline 4340 & 2 & 61 & 34.7279 & 16.54846 \\
\hline 4350 & 2 & 61 & 35.1472 & 16.74024 \\
\hline 4360 & 2 & 6? & 35.5713 & 10.9504 \\
\hline 4374 & 2 & 33 & 36.10049 & 17.15006 \\
\hline 4360 & 2 & 64 & 30.4350 & 17.36216 \\
\hline 4390 & ? & $6 \%$ & 36.8754 & 17.5717 \\
\hline
\end{tabular}


TABLE A31. (Continued 9)

\begin{tabular}{|c|c|c|c|c|}
\hline 4400 & 2 & 66 & 37,3206 & 17,78389 \\
\hline 4410 & 2 & 57 & 37.7711 & 17.99858 \\
\hline 4424 & 2 & 58 & 33.2271 & 18.21587 \\
\hline 4430 & 2 & 69 & 33,0336 & 18.43573 \\
\hline 44411 & 2 & 70 & 39,1530 & 16,65034 \\
\hline 44511 & $?$ & 71 & 39.6233 & 10.80359 \\
\hline 400 & 2 & 72 & 40.1067 & 19.11156 \\
\hline 4470 & 2 & 73 & 40.5909 & $19,3422 \pi$ \\
\hline 4480 & 2 & 74 & 41.0809 & 19.51578 \\
\hline 4491 & 2 & 75 & 41,5709 & 19.81211 \\
\hline 4500 & 2 & 76 & 42.0788 & 20.05128 \\
\hline 4510 & 2 & $7 ?$ & 42.5968 & $20,293.35$ \\
\hline 4520 & $?$ & 78 & 43.1009 & 20.53834 \\
\hline $453 !$ & 2 & 79 & $43,621 ?$ & 20,75028 \\
\hline 4540 & 2 & 80 & 44.1479 & 21.03722 \\
\hline 4550 & 2 & 81 & 44.5503 & $21.2 \times 119$ \\
\hline $456 u$ & 2 & 82 & 45.2202 & 21.54622 \\
\hline 4570 & 2 & 83 & 45.7601 & 21.00036 \\
\hline 4580 & 2 & 84 & 40.3150 & 22.07103 \\
\hline 4590 & 2 & $8 ;$ & $46.8977 \pi$ & 22.33300 \\
\hline 4000 & 2 & 86 & 47.4437 & 22.60770 \\
\hline 4610 & 2 & 87 & $40.1) 165$ & 22,88063 \\
\hline 4620 & 2 & 33 & 40.5762 & 23.1569 .1 \\
\hline 4630 & 2 & 89 & 49,1328 & 23,43647 \\
\hline 4640 & 2 & 913 & 49.7760 & 23.71940 \\
\hline 4650 & 2 & 91 & 50.3775 & 24.00574 \\
\hline 40611 & 2 & 92 & 50.9857 & 24,29555 \\
\hline $467 \mathrm{~J}$ & 2 & 93 & 51.61512 & 24.28835 \\
\hline 4680 & 2 & 94 & 95.3000 & 52,85634 \\
\hline 4690 & 2 & 95 & 86.8326 & 53.47494 \\
\hline 4700 & 2 & 36 & 87.3841 & 54.14075 \\
\hline 4710 & 2 & 97 & 88,7401 & 54.79435 \\
\hline 4720 & 2 & 38 & 90.0130 & 20.45504 \\
\hline 4735 & 2 & 99 & +1.1056 & 26.12532 \\
\hline 4740 & 2 & 100 & 92.2054 & 50.80289 \\
\hline 4750 & 2 & 101 & 93.3130 & 57.45003 \\
\hline 4760 & 2 & 102 & 94.4451 & 50.18205 \\
\hline 4770 & 2 & 103 & 95.5853 & 53.96504 \\
\hline 4780 & 2 & 104 & $95.739 ?$ & 59.59592 \\
\hline 4791 & 2 & 105 & 97,9071 & 00.31538 \\
\hline 4800 & 2 & 100 & 99.0891 & 01.104353 \\
\hline 4810 & 2 & 107 & 100.2853 & 51.78046 \\
\hline 4820 & 2 & 108 & 101.4300 & 02.52029 \\
\hline 4830 & 2 & 129 & 102.7213 & 63.20113 \\
\hline 4840 & 2 & 110 & 103.9613 & 64,04507 \\
\hline 4350 & 2 & 111 & 105.2104 & 04,31025 \\
\hline 4860 & 2 & 112 & 105.4066 & 65.00075 \\
\hline 4873 & 2 & 113 & 107.7721 & 05.39270 \\
\hline 4889 & $?$ & 124 & $109.073 ?$ & 67,19421 \\
\hline 4890 & 2 & 115 & 110.3399 & 63.00540 \\
\hline 4900 & 2 & 116 & 111.7220 & 03.82638 \\
\hline 4911$]$ & 2 & 117 & 113.0713 & $0 \% .65727$ \\
\hline 4920 & 2 & 118 & 114.4364 & 70.49020 \\
\hline 4933 & 2 & 119 & 115.8177 & 71.34927 \\
\hline
\end{tabular}


TABLE A31. (Continued 12)

\begin{tabular}{|c|c|c|c|c|}
\hline 6020 & 2 & 228 & 14.13789 & 11,38163 \\
\hline 010313 & ? & 229 & 13.8410 & 11.18983 \\
\hline $6,34 v$ & 2 & 23.3 & 13.5083 & 11,010121 \\
\hline 6050 & 2 & 231 & 1.3 .3709 & \pm 0.01577 \\
\hline $006: 1$ & 2 & 2.32 & 13.1534 & 10.63345 \\
\hline 0070 & 2 & 233 & 12.9317 & 10.45421 \\
\hline 6080 & ? & 2.34 & 12.7137 & 10.27790 \\
\hline 0090 & 2 & 235 & 12.4794 & 10.10474 \\
\hline 0100 & 2 & 236 & 12.2387 & $9,904+1$ \\
\hline 6110 & ? & 237 & $1<.0915$ & 9,16096 \\
\hline 0180 & 2 & 238 & 11.8779 & 9.603 .32 \\
\hline 61311 & 2 & 2.39 & 12.0717 & 9.44646 \\
\hline $014 \mathrm{~J}$ & 2 & 240 & 11.4300 & 4,26133 \\
\hline 6150 & 2 & 249 & 11.2575 & $9.124 \times 8$ \\
\hline 0104 & 2 & 2.42 & 11.070 & 0.97107 \\
\hline $61.7 \%$ & 2 & 243 & $10.910 \mathrm{j}$ & 6.319135 \\
\hline 6180 & ? & 244 & 3.2100 & 1.98450 \\
\hline 6190 & 2 & 245 & 3.1567 & 1.95142 \\
\hline 6200 & 2 & 246 & 3.105. & 1.91853 \\
\hline 021.0 & 2 & $\therefore 47$ & 3.0512 & $1,300 \pm 9$ \\
\hline 0220 & 2 & $? 248$ & $2.49+3$ & $1.5544 \mathrm{~J}$ \\
\hline 6230 & $?$ & 243 & 2.9492 & 1.32 .514 \\
\hline 6240 & 2 & 250 & $2.94,5$ & $1.79<41$ \\
\hline 6250 & 2 & 25. & 2. 35116 & 1.70219 \\
\hline 02611 & 2 & 252 & 2.8020 & 1.73249 \\
\hline 6270 & 2 & 25.5 & 2.7553 & 1.70529 \\
\hline 6260 & 2 & 254 & 2.7034 & 1.67458 \\
\hline 0294 & $?$ & 355 & 2.0532 & 1.04635 \\
\hline 6300 & 2 & 256 & 2.0185 & 1.51800 \\
\hline 0.310 & ? & 257 & 2.5742 & $1.5 \vee 161$. \\
\hline $0.32 . \mathrm{J}$ & $?$ & 253 & $2.5,500$ & 1.56449 \\
\hline 03313 & 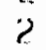 & 259 & $\ddot{2} .43 \times 1$ & 1.53012 \\
\hline 0340 & 2 & 260 & $? .4462$ & 1.21217 \\
\hline 0350 & 2 & 251 & 2.4050 & 1.40070 \\
\hline 0.360 & 2 & 262 & $2,36+4$ & 1.46164 \\
\hline 6370 & 2 & 263 & 2.3240 & 1.43700 \\
\hline 0380 & 2 & 254 & 2.2824 & 1.41278 \\
\hline 0390 & 2 & 265 & $? .2409$ & 1.30897 \\
\hline 6400 & 2 & 260 & 2.2050 & 1.56355 \\
\hline 6410 & 2 & 267 & $2 .\lfloor 717$ & 1.34254 \\
\hline 6420 & 2 & 200 & 2.1351 & 1.31990 \\
\hline 0430 & ? & 269 & 2.6991 & 1.29765 \\
\hline 6440 & 2 & 270 & 2.0036 & 1.27573 \\
\hline 6450 & ? & $27 i$ & $=.0290$ & 1.25428 \\
\hline 0460 & 2 & 272 & 1.9040 & 1.23313 \\
\hline 0470 & 2 & 273 & 1.9612 & $1.2123 \%$ \\
\hline 6480 & 2 & 2.74 & 1.9281 & i. 19191 \\
\hline 6490 & ? & 275 & 1.0956 & 1.17182 \\
\hline 0504 & 2 & 276 & 1.8030 & 1.15207 \\
\hline 6510 & ? & 277 & $1.832 ?$ & 1.13265 \\
\hline 6520 & 2 & 278 & 1.8010 & 1.11356 \\
\hline 6530 & 2 & 279 & 1.7710 & 1. 09479 \\
\hline 6540 & 2 & 201 & 1.7411 & 1.07033 \\
\hline 0550 & 2 & 281 & 1.7110 & 1.15519 \\
\hline
\end{tabular}


IABLE A31. (Cont inued 13)

\begin{tabular}{|c|c|c|c|c|c|}
\hline & 0560 & 2 & 232 & 1.6327 & $\begin{array}{r}1.04035 \\
1.02282\end{array}$ \\
\hline & $657 \mathrm{U}$ & 2 & 233 & 1.6546 & $1.0228 \%$ \\
\hline . & 6580 & 2 & 284 & 1.6267 & 1.00558 \\
\hline \multirow[t]{4}{*}{. } & 0590 & 2 & $20 \%$ & 1,5992 & .30862 \\
\hline & 6600 & 2 & 236 & 1.5723 & .97196 \\
\hline & 0610 & 2 & $23 \%$ & 1.5458 & .95558 \\
\hline & 6620 & 2 & 288 & 1.5197 &, 93947 \\
\hline \multirow[t]{13}{*}{ - } & 0630 & 2 & 289 & 1.4941 & .92353 \\
\hline & 6040 & 2 & 291 & 1.4639 & .90806 \\
\hline & 0050 & 2 & 291. & 1.4442 & .09276 \\
\hline & 6660 & 2 & 292 & 1. 4198 & .87771 \\
\hline & 6070 & 2 & 293 & 1.3959 & .86291 \\
\hline & 6080 & 2 & 291 & 6.2133 & $4,2752.4$ \\
\hline & 6090 & ? & 295 & 6.203 .3 & 4,32035 \\
\hline & 0700 & $?$ & 236 & $6.30+2$ & 4.37909 \\
\hline & 6710 & $?$ & 2.77 & $0.441 U$ & 4.45195 \\
\hline & 6720 & 2 & 293 & 6.5180 & 4.48546 \\
\hline & 6730 & 2 & 299 & 6.5973 & 4.53961 \\
\hline & 0740 & 2 & 390 & 0.0771 & 4.59441 \\
\hline & 6750 & 2 & 301 & 0.7578 & 4,64998 \\
\hline \multirow[t]{3}{*}{ - } & 6760 & 2 & 302 & 0.8333 & 4.70001 \\
\hline & 0770 & 2 & 303 & 6.9219 & 4.16282 \\
\hline & 6786 & 2 & 304 & 7.0055 & 4.82032 \\
\hline \multirow[t]{16}{*}{. } & 0790 & $\therefore$ & 305 & 7,0900 & 4.87851 \\
\hline & 6804 & 2 & 306 & $7.1 \% 50$ & 4,93741 \\
\hline & 6010 & 2 & 307 & 7.2623 & 4,99701 \\
\hline & 0324 & 2 & 3i) & 1.3499 & 5.135734 \\
\hline & 00330 & 2 & 309 & 7.4387 & 5.11839 \\
\hline & 6840 & 2 & 310 & 7.5285 & 5,181118 \\
\hline & 6850 & 2 & 311 & 7.6193 & 5,24272 \\
\hline & 6800 & 2 & 312 & 7.7113 & 5.30601 \\
\hline & 0870 & 2 & 313 & 7.8044 & 5.37007 \\
\hline & 0860 & 2 & 314 & 7,8080 & $b .43490$ \\
\hline & 0890 & 2 & 315 & 7,9940 & 5.50051 \\
\hline & 6900 & 2 & 316 & 3.0905 & 5.56691 \\
\hline & 6910 & 2 & 317 & 3,1882 & 5.63412 \\
\hline & 6920 & 2 & 318 & 4.2870 & 5,10213 \\
\hline & 6930 & 2 & 319 & $\overline{6} .3871$ & 5.77097 \\
\hline & $6941]$ & 2 & 320 & 3.4303 & 5.34064 \\
\hline \multirow[t]{3}{*}{. } & 6950 & 2 & 321 & 3.5900 & 5.91115 \\
\hline & 6960 & 2 & 322 & 4.0945 & 5.78251 \\
\hline & 6970 & 2 & 323 & 6.7995 & 6.05474 \\
\hline & 6980 & $\ddot{2}$ & 324 & 8.9057 & 0.1278 .3 \\
\hline & $694 \mathrm{~J}$ & $?$ & 325 & 9.11132 & 6.2 ㄴ. 101 \\
\hline & 7000 & 2 & 326 & 4.1220 & 0.27068 \\
\hline \multirow{3}{*}{8} & 7010 & 2 & 327 & 3.2321 & 6.35245 \\
\hline & 7020 & 2 & 323 & 9.3436 & 6.42914 \\
\hline & 7030 & 2 & 329 & 9.4564 & 0.50075 \\
\hline \multirow{6}{*}{ 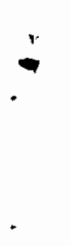 } & 7040 & 2 & 330 & 9.5705 & 0.58530 \\
\hline & 7050 & 2 & 331 & 7.6861 & 0.66483 \\
\hline & 7060 & $\overline{2}$ & 332 & 4.8030 & 0.74526 \\
\hline & 7070 & 2 & 333 & 9.9214 & 6.82669 \\
\hline & 7080 & 2 & 334 & 10.0411 & 6,70911 \\
\hline & 7090 & 2 & 335 & 10.1624 & 0.99252 \\
\hline
\end{tabular}


TABLE A31. (Continued 14)

\begin{tabular}{|c|c|c|c|c|}
\hline 7100 & 2 & 336 & 10.2850 & 7,07693 \\
\hline 7110 & 2 & 337 & 11.4092 & 7.16231 \\
\hline 7120 & 2 & 338 & 10.5349 & 7.24083 \\
\hline 7130 & 2 & 337 & 10.6020 & 7.33034 \\
\hline 7140 & 2 & 340 & 10.7903 & 7.42491 \\
\hline 7150 & 2 & 341 & 10.9210 & 7.51455 \\
\hline 7160 & 2 & 342 & 11.0529 & 7.02020 \\
\hline $71 \%$ & $?$ & 343 & 11.1 .303 & 7.69708 \\
\hline 7181 & 2 & 344 & 11.3213 & 7.79000 \\
\hline 7190 & ? & 345 & 11.4500 & 7.08404 \\
\hline 7200 & 2 & 375 & 11.3763 & 7.97922 \\
\hline 7210 & $?$ & 347 & 11.7303 & 8,07555 \\
\hline 7220 & 2 & 348 & 11.8780 & 5.11304 \\
\hline $723 \mathrm{~J}$ & 2 & 340 & 12.1214 & 0.27171 \\
\hline 7240 & 2 & 350 & 12.1063 & 6.37156 \\
\hline 7200 & ? & 321 & 12.3134 & 9.47203 \\
\hline 7260 & 2 & 352 & 12.4021 & 0.57491 \\
\hline 7270 & 2 & 353 & 12.5120 & 0.6784 \\
\hline 7200 & ? & 354 & 12.7048 & 8.78320 \\
\hline 7290 & $?$ & 352 & 12.9139 & 8.3892 \\
\hline 7300 & 2 & 350 & 13.3748 & 0.9365 \\
\hline 7310 & 2 & 357 & 13.2327 & 9,1051 \\
\hline 7320 & 2 & 358 & 13.3924 & 9.2150 \\
\hline 7330 & 2 & 359 & 13.5541 & 9.32632 \\
\hline 7.340 & 2 & 360 & 13.7177 & 9.7389 \\
\hline 7350 & 2 & 361 & 13.31935 & 9.5528 \\
\hline 7360 & 2 & 362 & 14, 14, & 9.6681 \\
\hline $737 \mathrm{~J}$ & 2 & 363 & 14,2206 & 9.7849 \\
\hline 7300 & 2 & 304 & 14.3923 & 9.9030 \\
\hline 7390 & 2 & 3.55 & 14.5600 & 10.0225 \\
\hline 7400 & 2 & 306 & 14,7418 & 10,1435 \\
\hline 1411 & 3 & 1 & 14.9193 & 10.2660 \\
\hline 7420 & 3 & 2 & 15.0999 & 10.3849 \\
\hline 7430 & 3 & 3 & 15.2822 & $1 \cup, 5154$ \\
\hline $744 J$ & 3 & 4 & 15.4667 & 10.6423 \\
\hline 7450 & 3 & 5 & 15.6534 & 10.7708 \\
\hline 7460 & 3 & 6 & 15.8424 & 14.9008 \\
\hline $747 i$ & 3 & 7 & 10.0337 & 11,0324 \\
\hline 7480 & 3 & 3 & 16.2272 & 11,1656 \\
\hline 7490 & 3 & $\exists$ & 13,4231 & 11,3004 \\
\hline 7500 & 3 & 10 & 16.6214 & 11,4308 \\
\hline 7510 & 3 & 11 & 16.3220 & 11.5749 \\
\hline 7520 & $j$ & $1 ?$ & 17.0251 & 11.7146 \\
\hline 7531 & $j$ & 13 & 17,2307 & 11.0500 \\
\hline 7540 & 3 & 14 & 17,4367 & 11.9942 \\
\hline 7550 & 3 & 15 & 17.0492 & 12.1440 \\
\hline 7560 & 3 & 16 & 17.8623 & 12.2900 \\
\hline 7570 & 3 & 17 & 18.13779 & 12.4590 \\
\hline 7580 & 3 & 10 & 10.2961 & 12.58922 \\
\hline 7590 & 3 & 19 & 10.5170 & 12,74120 \\
\hline 7000 & 3 & $2 J$ & 10.7406 & 12.0950 \\
\hline 7610 & 3 & 21 & 13.9608 & 13,0506 \\
\hline 7020 & 3 & 22 & 19.1956 & 15,2082 \\
\hline 7631 & 3 & 23 & 19.4275 & 13.3676 \\
\hline
\end{tabular}


TABLE A31. (Continued 15)

\begin{tabular}{|c|c|c|c|c|}
\hline 7040 & 3 & 24 & 19.0620 & 13.52907 \\
\hline 7ヒつい & 3 & ?इ & 19.8994 & 13.09240 \\
\hline $760: 1$ & 3 & 20 & 20.1396 & 13.85769 \\
\hline 7670 & 3 & 27 & 20,3820 & 14.02499 \\
\hline 7680 & 3 & 28 & 20,5280 & 14.19430 \\
\hline 7690 & 3 & 27 & 20,8779 & 14.30566 \\
\hline 7700 & 3 & 30 & 21.1279 & 14.53909 \\
\hline $771 \mathrm{j}$ & 3 & 31 & 21.3350 & 14.71461 \\
\hline 7720 & 3 & 32 & 21.6432 & 14.89225 \\
\hline 77511 & $s$ & 33 & .0654 & 8.03299 \\
\hline $774 !$ & 3 & 34 & .0072 & 8.12997 \\
\hline 7730 & 3 & 35 & .0680 & 8,22012 \\
\hline $770 u$ & 3 & 30 & .0608 & 8.32745 \\
\hline 7770 & 3 & 37 & $.009 ?$ & 3.42798 \\
\hline $77: 0$ & 3 & 35 & خט & 8.52973 \\
\hline 7790 & 3 & 39 &, 0713 & 0.03270 \\
\hline 7800 & 3 & 40 & .0722 & 0.73592 \\
\hline 7010 & 3 & 41 & .0731 & 0.84239 \\
\hline $78 \%$ & 3 & 42 & .0790 & 0.94914 \\
\hline 7830 & 3 & 43 & .0749 & 9.05718 \\
\hline 7840 & $\ddot{u}$ & 44 & . & 9.15652 \\
\hline 7050 & 3 & $4 ' s$ & ומים & 9.27718 \\
\hline 7800 & 3 & 46 & .0776 & 9.38918 \\
\hline 7570 & 3 & 47 & $17 . ? 310$ & 12.43637 \\
\hline 7880 & 3 & 48 & 17.4300 & 12.58853 \\
\hline 70,900 & 3 & 49 & 17.0495 & 12.74050 \\
\hline 7901 & 3 & bu & 17.3526 & 12,49431 \\
\hline $79 \%$ & 3 & 51 & 18.0732 & 13.04997 \\
\hline 7920 & 3 & 52 & 13.2905 & 13,20751 \\
\hline 7934 & 3 & 53 & 10.5174 & 13,36696 \\
\hline 7940 & 3 & 54 & 18,1408 & 13.52833 \\
\hline 7450 & $j$ & 55 & 18,9572 & 13.65165 \\
\hline 7960 & 3 & 36 & 13.1961 & 13.85694 \\
\hline 7970 & s & 57 & 19.4279 & 14,02422 \\
\hline 7980 & 3 & 58 & 19.6024 & 14.19352 \\
\hline 7990 & 3 & 59 & 13.8990 & 14,36487 \\
\hline 0000 & 3 & 60 & 20.1400 & 14,53029 \\
\hline $801 \mathrm{U}$ & 3 & 01 & 20.3832 & 14.71380 \\
\hline 8025 & $s$ & 62 & 20,6292 & $14,8914.3$ \\
\hline $003 \mathrm{~J}$ & 3 & 63 & 20.4783 & 15.07120 \\
\hline 0040 & 3 & 64 & 21.1303 & 15.25315 \\
\hline 8050 & 3 & 65 & 21,3834 & 15.43729 \\
\hline 0400 & 3 & 66 & 21.6436 & 15.52365 \\
\hline 8070 & 3 & 67 & 21.9049 & 15,81226 \\
\hline 8080 & 3 & 68 & 22,1693 & 15,00315 \\
\hline 6บ9บ & 3 & 09 & 22.4370 & 16.19635 \\
\hline 0100 & 3 & 70 & 22.7073 & 16.39188 \\
\hline 8110 & 3 & 71 & 22,9820 & 10,50976 \\
\hline 8120 & 3 & 72 & 23,2534 & 16,79404 \\
\hline 0134 & 3 & 73 & 23.5402 & 16.99273 \\
\hline 8140 & 3 & 74 & 23.8244 & 17.19788 \\
\hline $8 \div 50$ & 3 & 75 & 24.1120 & 17.40549 \\
\hline $0 \pm t i$ & 3 & 76 & 24,4031 & 17.61562 \\
\hline 8170 & 3 & 77 & 24.5977 & 17,52028 \\
\hline
\end{tabular}


TABLE A31. (Continued 16)

\begin{tabular}{|c|c|c|c|c|}
\hline 8160 & 3 & 73 & 24,9950 & 15,04351 \\
\hline $619 \mathrm{~J}$ & $b$ & 79 & 25.2970 & 16,20133 \\
\hline $820 \mathrm{i}$ & 3 & $3 \mathrm{~J}$ & 25.6031 & 18.48179 \\
\hline 2210 & 3 & 31 & 25,9121 & 14.70491 \\
\hline 0220 & 3 & 82 & $20.2 ? .49$ & 10.93072 \\
\hline 8230 & 3 & 83 & 26.5415 & 19,15926 \\
\hline 8240 & 3 & 84 & 25.8619 & 19.39055 \\
\hline 8250 & 3 & 33 & 27.1802 & 19.62464 \\
\hline 2204 & 3 & 85 & 27.5144 & 18.00155 \\
\hline 6270 & 3 & 37 & 27.8466 & 20.10133 \\
\hline 6280 & 3 & 80 & 23.1827 & 20,34400 \\
\hline 8290 & 3 & 89 & 28.5230 & $<0.58960$ \\
\hline 0301$)$ & 3 & 20 & 20.8673 & 20.83816 \\
\hline $0.51 . j$ & 3 & 91 & $24.213 \theta$ & $<1.00972$ \\
\hline 8320 & 3 & 92 & 29.5085 & 21,34432 \\
\hline 0330 & 3 & 9.5 & 27.9225 & 21.00200 \\
\hline $834 \mathrm{~J}$ & 3 & 94 & 35.1269 & 24.74894 \\
\hline 8350 & 3 & 35 & 35.5508 & 20.04772 \\
\hline 8360 & 3 & 46 & 35.9801 & 23.35010 \\
\hline$\$ 370$ & 3 & 97 & 36.4145 & 25.55613 \\
\hline $\operatorname{css} 0$ & 3 & 98 & 35.3541 & 22,90586 \\
\hline 8304 & 3 & 97 & 37.2390 & 20.27933 \\
\hline 8400 & 3 & 100 & 37.7493 & 20.58658 \\
\hline 8410 & 3 & 101 & 30.2020 & 20.91766 \\
\hline 6420 & 3 & 102 & 33.0662 & 21.242 .62 \\
\hline $843 !$ & 3 & 103 & 39.1330 & 27.57150 \\
\hline 8440 & 3 & 101 & 33.0004 & $27.9 \cup 435$ \\
\hline 8450 & $j$ & 105 & 40.0330 & 20.24123 \\
\hline 0460 & 3 & 105 & 40.5673 & 20.58216 \\
\hline 8473 & 3 & 107 & 41.0572 & 28.92721 \\
\hline 8480 & 3 & 108 & 41.5529 & 29.27043 \\
\hline 0490 & 3 & 109 & 42.0545 & 29.62986 \\
\hline $050 \hat{0}$ & 3 & 110 & 42.5622 & 29.90750 \\
\hline 3510 & 3 & 111 & 43,0760 & 30.34958 \\
\hline 8520 & 3 & $11 ?$ & 43.5960 & 30.71597 \\
\hline 0534 & 3 & 113 & 44.1223 & 31,08678 \\
\hline 0540 & 3 & 114 & 44,6550 & 31.46207 \\
\hline 8550 & 3 & 115 & $4 \vdots .1741$ & 30.93174 \\
\hline لي 850 & 3 & 116 & 15.7397 & 30.41037 \\
\hline 8570 & 3 & 117 & 46.2919 & 29.09773 \\
\hline $858 \mathrm{a}$ & 3 & 118 & 40.3507 & 29.39377 \\
\hline 8590 & 3 & 119 & 47,4103 & 26.09030 \\
\hline 8600 & 3 & 120 & 47.9857 & 28.41113 \\
\hline $86 i 0$ & 3 & 121 & 43.5501 & 27.93227 \\
\hline 3620 & $j$ & $1 \geq 2$ & 41,7494 & 27.46144 \\
\hline 3636 & 3 & 123 & 46.9445 & 20.99654 \\
\hline 8040 & 3 & 124 & 46.1332 & 26.54344 \\
\hline 0650 & 3 & 222 & 45.3752 & 20,09602 \\
\hline $86 t .4$ & 3 & 126 & $44,61,14$ & 26.65613 \\
\hline 8070 & 3 & 127 & 43.8587 & 23.22367 \\
\hline 0080 & 3 & 125 & 45.1 .23 & 24.79819 \\
\hline 0090 & 3 & 123 & 42,3423 & $24.3 d \div 48$ \\
\hline $870 \mathrm{j}$ & 3 & $13 i$ & 41.5777 & 25.9095 \\
\hline $8 \% 1 !$ & 3 & 131 & 11.9752 & 23.56547 \\
\hline
\end{tabular}


TABLE A31. (Continued 17)

\begin{tabular}{|c|c|c|c|c|}
\hline 3720 & 3 & 132 & 40,2845 & 25,16825 \\
\hline 8730 & j & 133 & 39.61154 & 22.77772 \\
\hline 874.1 & 3 & 134 & 38.9378 & 22.54377 \\
\hline 750 & j & 135 & 33.2315 & 22.01029 \\
\hline 6700 & 3 & 136 & 31.5168 & 13.32731 \\
\hline 8770 & 3 & 137 & $30,985 b$ & 18,50995 \\
\hline $878 \mathrm{~J}$ & 3 & 139 & 30.4532 & 18.19794 \\
\hline 0790 & 3 & 137 & 29,0497 & 17.89119 \\
\hline 0860 & $j$ & $14 j$ & 29,4449 & 17.58401 \\
\hline 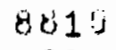 & 5 & $\$ 41$ & 20.9186 & 17.29312 \\
\hline 8020 & 3 & 142 & 28.4606 & 17.00162 \\
\hline 8830 & 3 & 143 & 27.9600 & 10.71503 \\
\hline $884 i$ & 3 & 1.44 & 27.5092 & 16.43328 \\
\hline 8850 & 3 & 145 & 27.0455 & 10.15628 \\
\hline $086:$ & 3 & 140 & 20.5090 & 15,48394 \\
\hline $88 i 0$ & 3 & 147 & 26.1414 & 15,61620 \\
\hline 8080 & 3 & 148 & 25,7008 & 15,35296 \\
\hline 8090 & 3 & 149 & 25.2675 & $1 \%, 09417$ \\
\hline 3900 & 3 & 150 & 24.3416 & 14.03974 \\
\hline 6910 & 3 & 151 & 24.4220 & 14.58959 \\
\hline 0960 & 3 & 152 & 24,0112 & 14,34367 \\
\hline 0934 & 3 & 153 & 23.0065 & 14.10187 \\
\hline $694 !$ & 3 & 154 & 23,2005 & 13,86418 \\
\hline $890^{\circ}$ & 3 & 155 & 22.8173 & 13.63048 \\
\hline 0960 & 3 & 156 & 22.4327 & 13,40072 \\
\hline 8971 & 3 & 157 & 22.0546 & 13,17483 \\
\hline $698 \mathrm{~J}$ & 3 & 158 & 21.0824 & 12.95276 \\
\hline 09511 & 3 & $15 \%$ & 21.3173 & 12,75442 \\
\hline 9000 & 3 & 160 & 20.9550 & 12,51977 \\
\hline 901:- & 3 & 161 & 20.6047 & 12.30873 \\
\hline $902 i^{2}$ & 3 & 102 & 20.2574 & 12,10125 \\
\hline 9030 & 3 & 163 & 14.9159 & 11.39720 \\
\hline 9041 & 3 & 164 & 19.5802 & 11.09072 \\
\hline 90513 & 3 & 163 & 19,2502 & 11.49956 \\
\hline 9060 & 3 & 106 & 18.9257 & 11,30572 \\
\hline 90,70 & 3 & 167 & 18.6067 & 11,11514 \\
\hline 4080 & $\ddot{3}$ & 168 & 10.2031 & 10.92778 \\
\hline 90911 & 3 & 167 & 17,9647 & 10.74358 \\
\hline $910 !$ & 3 & 170 & 17.6315 & 14,50248 \\
\hline 4110 & 3 & 171 & 17.3835 & 10,38444 \\
\hline 9180 & 3 & 172 & 17,0205 & 10.23940 \\
\hline 9130 & 3 & 173 & 16.8024 & 10.03730 \\
\hline $914 i$ & 3 & 174 & 16.5191 & 9.85011 \\
\hline 9150 & 3 & 175 & 4.0423 & 4.54123 \\
\hline$\$ 100$ & 3 & 176 & 8.8099 & 4.42530 \\
\hline $917 v$ & 3 & 177 & 3.7400 & 4.35076 \\
\hline $918 \mathrm{~L}$ & 3 & 178 & 0.5927 & 4.27743 \\
\hline 9194 & 3 & 179 & 8,4479 & 4.20532 \\
\hline $920 \mathrm{U}$ & 3 & 130 & 3.3055 & 4.13444 \\
\hline 9210 & j & 181 & 9.1655 & 4.06475 \\
\hline 9221 & 3 & 102 & 0.0272 & 5.99623 \\
\hline$\$ 230$ & $j$ & 183 & 7.8925 & 3.40807 \\
\hline 9240 & 3 & 184 & 7.7592 & 5.00264 \\
\hline 50 & 3 & 135 & 7.0287 & 3.79753 \\
\hline
\end{tabular}


TABLE A31. (Continued 18)

\begin{tabular}{|c|c|c|c|c|}
\hline 92610 & 3 & 186 & 7.5001 & 3,73352 \\
\hline $927:$ & 3 & is 7 & 7.3737 & 3.67459 \\
\hline 4280 & $j$ & 1933 & $7.24 \times 1$ & 5.60871 \\
\hline 9290 & 3 & 189 & 7.1272 & 3,54788 \\
\hline 9300 & 3 & $191 j$ & 7.0070 & 3.40808 \\
\hline 931.0 & 3 & 191 & 2. & 3.42928 \\
\hline 9320 & 3 & 192 & 0.7728 & 3.37143 \\
\hline 93311 & 3 & 125 & 0.0506 & 3.51405 \\
\hline 9.540 & 3 & 194 & 0.5464 & 3.25878 \\
\hline 3350 & 0 & 193 & 0.4360 & .5 .20384 \\
\hline $936 !$ & 3 & 146 & 5.3275 & $5.14 \times 84$ \\
\hline 3371 & 3 & 197 & 6.2702 & 3.09074 \\
\hline 4381 & 3 & 190 & 0.1101 & 0,124455 \\
\hline 4590 & 3 & 139 & 0,1129 & 2,44323 \\
\hline 941,0 & 3 & 200 & 5.9116 & 2.44277 \\
\hline 9410 & 3 & 201 & 5.8119 & 2.39317 \\
\hline 9420 & 3 & 202 & 5.7140 & 2.844415 \\
\hline 4431 & 3 & 203 & $5,0.77$ & 2.79645 \\
\hline $9441 j$ & 3 & 204 & 2.52 .30 & 2,74931 \\
\hline $94 b:$ & 3 & 205 & 5.4279 & 2.70247 \\
\hline 9460 & 3 & ?:15 & 5.3303 & 2.55741 \\
\hline$\$ 470$ & $S$ & 207 & 7.2071 & $4.22+22$ \\
\hline 946.3 & 3 & 208 & 7.2446 & 4.15302 \\
\hline y4901 & 3 & 209 & $7 .(1242$. & 4,00301 \\
\hline 9501 & 3 & 210 & 0.9053 & 4,01419 \\
\hline 9511 & 3 & 211 & 6.7891 & $3.9+553$ \\
\hline 4501 & 3 & $21 ?$ & 0.0749 & 3.08600 \\
\hline 9530 & 3 & 213 & 0.5524 & $\therefore .81400$ \\
\hline $954 \mathrm{U}$ & 3 & 211 & 6.4510 & $3.75 \cup 30$ \\
\hline 9554 & 3 & 215 & 5.3430 & 3.60708 \\
\hline 2561 & 3 & 210 & 6.2361 & 3.62493 \\
\hline $957:$ & 3 & 217 & $0.13: 0$ & 3.56333 \\
\hline 95611 & 3 & $? 18$ & 2,0270 & 5.50370 \\
\hline 9590 & $s$ & 217 & 3.9360 & 3.44470 \\
\hline 9600 & 3 & $22 !$ & $5.8 ? 62$ & 3.38663 \\
\hline 9614 & 3 & 221. & b.7279 & 3.32954 \\
\hline 9020 & 3 & 22? & 5.0 .314 & 3,27342 \\
\hline 9630 & 3 & 223 & $5.536 \%$ & 3.21624 \\
\hline $464 i j$ & 3 & 224 & 5.4431 & 3,10400 \\
\hline 965.6 & 3 & 225 & 5.3514 & 3,11066 \\
\hline 9660 & 3 & 226 & 3.2612 & 3.05823 \\
\hline 3674 & 3 & 227 & 5.1725 & 3,00060 \\
\hline 9080 & 3 & 220 & 5.0853 & 2,95000 \\
\hline $90 \div 1 \mathrm{j}$ & 5 & 229 & 4.9595 & 2,90617 \\
\hline 9701 & $\ddot{3}$ & 230 & $4,413 j$ & 2.85710 \\
\hline 9711 & 3 & 231 & 4.8325 & 2.80402 \\
\hline 4720 & 3 & 232 & 4.7510 & 2.70167 \\
\hline 9731 & 3 & 233 & 4.6709 & 2,7151 \\
\hline 9740 & 3 & 234 & 4.5022 & 2.66935 \\
\hline 9750 & 3 & 235 & 4.5148 & 2.6243 \\
\hline 9760 & 3 & 236 & 4.4307 & 2.56412 \\
\hline 9770 & 3 & 237 & 4,3039 & 2.53603 \\
\hline 9700 & 3 & 238 & 4.2 .903 & 2,49387 \\
\hline 9790 & 3 & 239 & 4.2130 & 2.4518 \\
\hline
\end{tabular}


TABLE A31. (Continued 19)

\begin{tabular}{|c|c|c|c|c|}
\hline 9800 & 3 & 240 & 4,1469 & 2.41050 \\
\hline 9810 & 3 & 241 & 4,0770 & 2,36987 \\
\hline 9620 & 3 & $2.4 ?$ & 4,0033 & 2.32992 \\
\hline 4830 & 5 & 2.43 & $s: 9407$ & 2.29065 \\
\hline 9840 & 3 & 244 & 9.0543 & 2.47289 \\
\hline 9850 & 3 & 245 & $\$ .7911$ & 2.43121 \\
\hline 9860 & 3 & 246 & 9.6264 & 2.39022 \\
\hline 9070 & 3 & 247 & 9,4641 & 2.34993 \\
\hline 9864 & 3 & 240 & 5.3046 & 2.31032 \\
\hline 9840 & 3 & 249 & 3.1477 & 2.27138 \\
\hline 9900 & 3 & 250 & 3.9935 & 2.23309 \\
\hline 9910 & 3 & 251 & 8.8419 & 2.14545 \\
\hline 9920 & 3 & 252 & 3.0423 & 2.15844 \\
\hline 9930 & 3 & 253 & 0.5464 & 2.12206 \\
\hline 9940 & 3 & 254 & 3.4023 & $2.900<9$ \\
\hline 9954 & 3 & 255 & 0.2537 & 2,05112 \\
\hline 9460 & 3 & 256 & 3,1214 & 2.01555 \\
\hline 9470 & 3 & 2.57 & $7.984 b$ & 1.98250 \\
\hline $998: 1$ & 3 & 258 & 7.8500 & 1.94914 \\
\hline $9 \div 90$ & 3 & 259 & 7.7170 & 1,91628 \\
\hline 10000 & 3 & 260 & 7.5875 & 1.80390 \\
\hline 10014 & 3 & 261 & 1.4595 & 1.85222 \\
\hline $100<0$ & 3 & 262 & 7.3339 & 1.02100 \\
\hline 10030 & 3 & 263 & 7.2103 & 1.79031 \\
\hline 10040 & 3 & 264 & 7.0887 & 1.76013 \\
\hline 10000 & 3 & 265 & 0.9692 & 1.73040 \\
\hline 10060 & 3 & 265 & $\therefore .8510$ & 1.70129 \\
\hline 10010 & 3 & 267 & 5.7363 & $1.67 \geqslant 61$ \\
\hline 10030 & 3 & 208 & 6.6227 & 1,64442 \\
\hline 10090 & 3 & 269 & 0.5111 & 1.61670 \\
\hline $101 \cup 0$ & 3 & 270 & 0,4013 & 1.58945 \\
\hline 10110 & 3 & 271 & 0.2934 & 1.26260 \\
\hline $101 \geq 0$ & 3 & 272 & 6.1874 & 1.23032 \\
\hline $1013 \mathrm{u}$ & 3 & 273 & 0.0831 & 1.51042 \\
\hline 10140 & 3 & 274 & 9,9605 & 1.40496 \\
\hline 10150 & 3 & 275 & 5.3797 & 1.45993 \\
\hline 10100 & 3 & 276 & 5.7806 & 1.43532 \\
\hline 10190 & 3 & 277 & 5.0832 & 1.41112 \\
\hline 10130 & 3 & 278 & 5.5874 & 1.30734 \\
\hline 10190 & 3 & 279 & 5.4932 & 1.35395 \\
\hline 10200 & 3 & 280 & 5.4006 & 1.34096 \\
\hline 10210 & 3 & 281 & 5,3096 & 1.31836 \\
\hline 10220 & 3 & 232 & 5.2201 & 1,29014 \\
\hline $1023 !$ & 3 & 283 & 5.1321 & 1.27429 \\
\hline 10240 & 3 & 284 & .0259 & .02594 \\
\hline 10230 & 3 & 235 & .11265 & .02020 \\
\hline $1 \cup 200$ & 3 & 286 & .0266 & .02657 \\
\hline 10270 & 3 & 287 & .0209 & .02089 \\
\hline 10280 & 3 & 280 & .0212 & .12722 \\
\hline 10290 & 3 & 289 & .0275 & .02755 \\
\hline 10300 & 3 & 290 & .0279 & .02763 \\
\hline 103111 & 3 & 291 & $.028 \mathrm{c}$ & .02322 \\
\hline 10320 & 3 & 292 & .0230 & .02856 \\
\hline 103319 & 3 & 293 & .0234 & $.028 \times 0$ \\
\hline
\end{tabular}


TABLE A37. (Continued 26)

\begin{tabular}{|c|c|c|c|c|}
\hline 13584 & 4 & 253 & 1.3630 & 1.12623 \\
\hline 13540 & 4 & 254 & 1.3408 & 1.10155 \\
\hline 13000 & 4 & S5 & 1.3182 & ]. 4827 \\
\hline 13010 & $\iota_{i}$ & 250 & 1.2560 & 1.0645 \\
\hline 13630 & 4 & 257 & 1.2741 & 1.0405 \\
\hline 13030 & 4 & 256 & 1.2526 & 1.0239 \\
\hline 13640 & 4 & 259 & \pm .2315 & 1.4110 \\
\hline 13650 & 4 & 264 & i. 2100 & .9045 \\
\hline 13060 & 4 & 201 & 1.19115 & .9277 \\
\hline $130 \%$ & is & 262 & i. 17013 & . $y \Delta 1$ \\
\hline 13654 & 4 & 263 & 1.1506 & .9451 \\
\hline 13694 & 4 & 264 & 1.1312 & .9201 \\
\hline 13700 & 4 & 265 & 1.1121 & .9135 \\
\hline $13 \%$ & 4 & $26 s$ & 1.0935 & .0901 \\
\hline 13720 & 4 & 201 & i. 0749 & .3625 \\
\hline 13736 & 4 & 208 & 1.0500 & .0060 \\
\hline 13740 & 4 & 209 & 1.0390 & .853 \\
\hline 13750 & 4 & 270 & 1.0215 & .2560 \\
\hline 13700 & 4 & 271 & 1.0043 & .8245 \\
\hline 10770 & 4 & $27 \hat{2}$ & .9873 & .0110 \\
\hline 13740 & 4 & 273 & .9707 & .7975 \\
\hline 13760 & 4 & 274 & .9543 & .7835 \\
\hline 13800 & 4 & 275 & .9382 & .7706 \\
\hline $138: 0$ & 4 & 276 & .9224 & $.75 \%$ \\
\hline $138<0$ & 4 & 277 & . 90064 & .7445 \\
\hline 13600 & 4 & 273 & .8916 & .7325 \\
\hline $10041 j$ & 4 & 279 & .8766 & .7200 \\
\hline 10500 & 4 & 280 & .8018 & .7075 \\
\hline 13600 & 4 & 281 & .8473 & .6955 \\
\hline 13670 & 1 & $2 \pi 2$ & .8530 & .0042 \\
\hline $138 \pi 0$ & 4 & 283 & .8137 & .6726 \\
\hline 135.90 & 4 & 284 & $.8 u b 1$ & .6013 \\
\hline 13509 & 4 & 235 & .7910 & .0202 \\
\hline 13910 & 4 & 200 & .7702 & .0392 \\
\hline 13324 & 4 & 267 & .5017 & .302 \\
\hline 13930 & 4 & 200 & .5077 & .3008 \\
\hline 13940 & 4 & 287 & .5139 & .371 \\
\hline 13950 & 4 & 290 & .5201 & .3758 \\
\hline 13900 & 4 & 291 & .5264 & .380 \\
\hline 13570 & 4 & 292 & .5327 & .384 \\
\hline 13900 & 4 & 293 & .5391 & .309 \\
\hline 13990 & 4 & 294 & .5456 & .3942 \\
\hline 140130 & 4 & 295 & .5522 & .3390 \\
\hline $1401 \mathrm{U}$ & 4 & 296 & .5580 & .4038 \\
\hline 14020 & 4 & 297 & .5556 & .408 \\
\hline 14036 & 4 & $2+5$ & .5725 & .4130 \\
\hline $14(40)$ & 4 & 290 & .5794 & .4160 \\
\hline 14056 & 4 & 300 & .5864 & .423 \\
\hline 14060 & 4 & 301 & .5933 & .428 \\
\hline 14070 & $\therefore$ & 302 & .6006 & .4340 \\
\hline 14000 & 7 & sis & .6079 & .434 \\
\hline 140.00 & 4 & 364 & .6152 & .4445 \\
\hline 14100 & 4 & $3 ! 15$ & .6226 & .4498 \\
\hline 14114 & 4 & 306 & .6302 & 4033 \\
\hline
\end{tabular}


TABLE A31. (Continued 27)

\begin{tabular}{|c|c|c|c|c|}
\hline 14120 & 4 & 307 & .6378 & $.46 \cup 85$ \\
\hline 14150 & 1 & 308 & .6435 & .46042 \\
\hline 14140 & 4 & 309 & .6533 & .47205 \\
\hline 14154 & 4 & 310 & .6611 & .4777 \\
\hline 14100 & 4 & 311 & .6091 & .4835 \\
\hline 14170 & 4 & $31 ?$ & .6772 & .4893 \\
\hline 14186 & 4 & 313 & .6854 & .4952 \\
\hline 14190 & 4 & 314 & .6937 & .5012 \\
\hline 14200 & 4 & 315 & .7020 & .5072 \\
\hline 14214 & 4 & 310 & .7105 & .5134 \\
\hline 14220 & 4 & $31 \%$ & .7191 & .5190 \\
\hline 142310 & 1 & 318 & .7278 & .5258 \\
\hline $1424 !$ & 4 & 319 & .7366 & .5322 \\
\hline 14250 & 4 & 320 & .7454 & .5380 \\
\hline 14200 & 4 & $32:$ & .7544 & .5451 \\
\hline $14 c 71$ & 4 & 322 & .7635 & .5217 \\
\hline 14600 & $a$ & 323 & .7728 & .0584 \\
\hline 14296 & 4 & 324 & .7821 & .2051 \\
\hline 14300 & 4 & 325 & .7915 & .5719 \\
\hline 14310 & 4 & 320 & .8011 & .5788 \\
\hline 14320 & 4 & 327 & .0100 & .5058 \\
\hline 14350 & 4 & 325 & .8240 & .5929 \\
\hline 14340 & 4 & 329 & .8305 & .6000 \\
\hline 14350 & 4 & 336 & .8405 & .0073 \\
\hline 14300 & 4 & 331 & .8506 & .61467 \\
\hline 14370 & 4 & 332 & .8009 & .0220 \\
\hline 14350 & 4 & 3.33 & .8715 & .6290 \\
\hline 14340 & 4 & 534 & .8813 & $.037 ?$ \\
\hline $1440 \mathrm{U}$ & 4 & 335 & .8925 & .6448 \\
\hline 14416 & 4 & 330 & .9032 & .6526 \\
\hline 14421$]$ & 4 & 337 & .9141 & .0065 \\
\hline 14430 & 4 & 338 & .9252 & .6085 \\
\hline 14446 & 4 & 334 & .9363 & .6766 \\
\hline $4 \Delta \geqslant U$ & 4 & 340 & .9476 & .6847 \\
\hline 4460 & 4 & 341 & .9581 & .6930 \\
\hline 14470 & 4 & 342 & .9707 & .7414 \\
\hline 14480 & 4 & 343 & .9824 & .7098 \\
\hline 14490 & 4 & 344 & .9942 & .7164 \\
\hline 14504 & 4 & 345 & 1,0062 & .7271 \\
\hline 14510 & 4 & 346 & 1.0184 & .7358 \\
\hline 14520 & 4 & 347 & 1.0307 & .7447 \\
\hline 14530 & 4 & 348 & 1.0431 & .7537 \\
\hline 14540 & 4 & 349 & 1.0557 & .7628 \\
\hline 14550 & 4 & 350 & 1.0005 & .7720 \\
\hline 14560 & 4 & 351 & 1.0814 & .7813 \\
\hline 14570 & 4 & 352 & 1.0944 & .7908 \\
\hline 14560 & 4 & 353 & 1.1076 & . \\
\hline 14590 & 4 & 354 & 1.1210 & .8100 \\
\hline 146.16 & 4 & 355 & 1.1345 & .8196 \\
\hline 14610 & 4 & 350 & 1,1432 & .8297 \\
\hline 14620 & 4 & 357 & 1.1621 & .3097 \\
\hline 14630 & 4 & 358 & 1.1761 & .0496 \\
\hline 14640 & 4 & 359 & 1.1933 & .8001 \\
\hline 14650 & 4 & 360 & 1.2047 & .874 \\
\hline
\end{tabular}


TABLE A31. (Continued 28)

$\begin{array}{lllll}14060 & 4 & 361 & 1.2192 & .85102 \\ 14670 & 4 & 360 & 1.2339 & .89160 \\ 14660 & 4 & 363 & 1.2406 & .90242 \\ 14690 & 4 & 364 & 1.2659 & .91331 \\ 14706 & 4 & 365 & 1.2792 & .92434\end{array}$

A. 166 


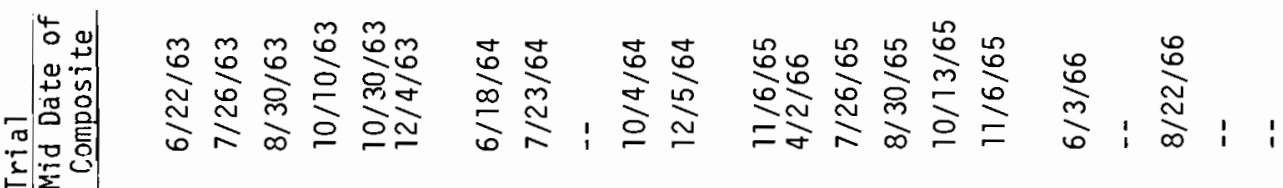

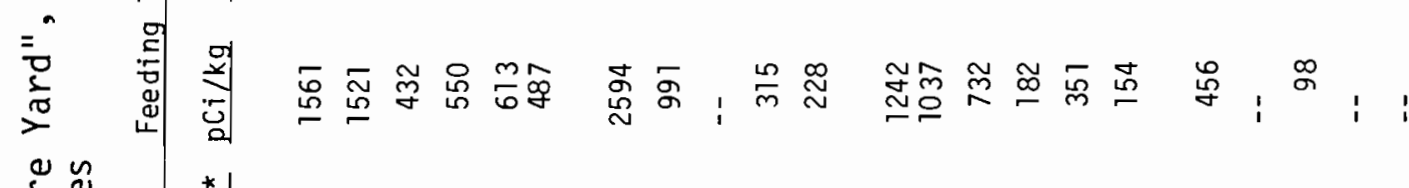

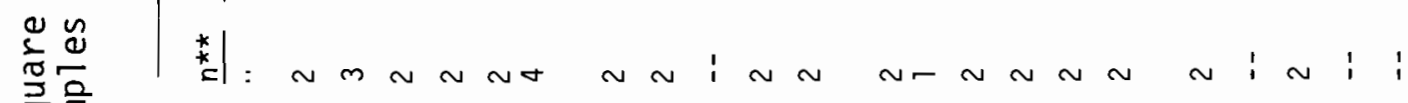

第翯

管重罚

:

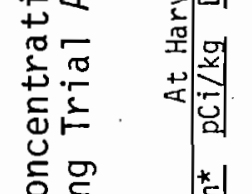

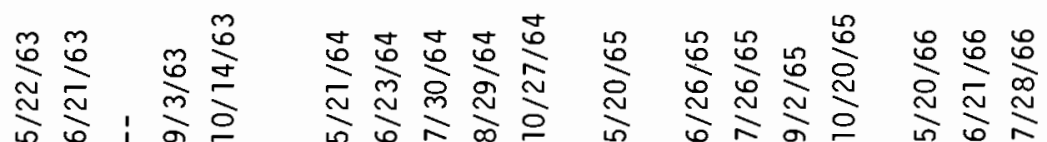

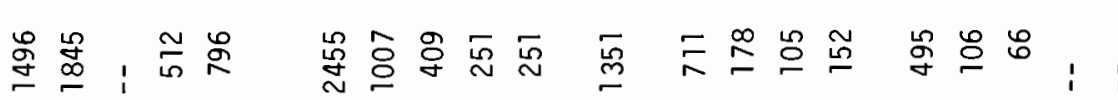

号.

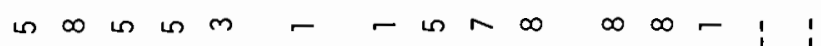

ษ

눈

$\rightarrow$ 은

4 宁

ᄃิ

ing

$\frac{\pi}{\pi}$

몽모

容苗

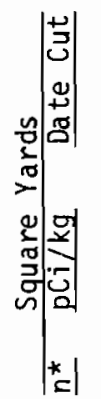

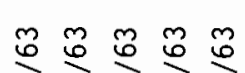

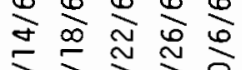

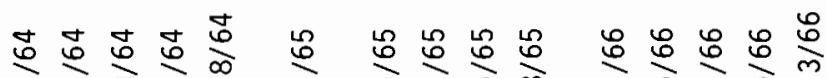

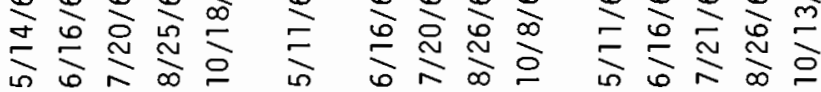

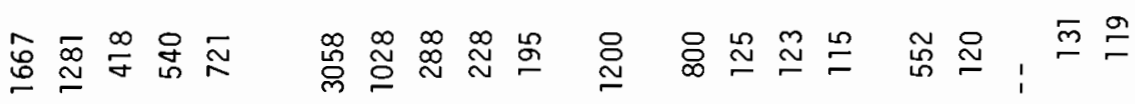

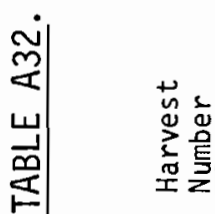

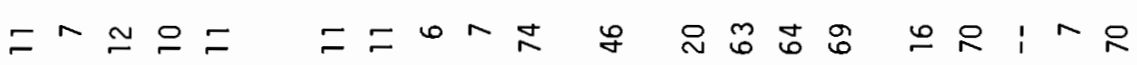

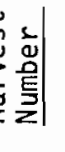

$-\infty m+\infty$

$-\sim m+\infty$

$-\sim m+i$

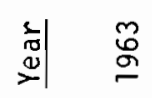

ఫั

㟔

\&̊

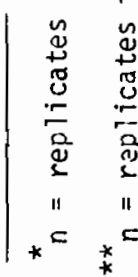


APPENDIX B 
LEO SYPHUS FARM

SAINT GEORGE, UTAH

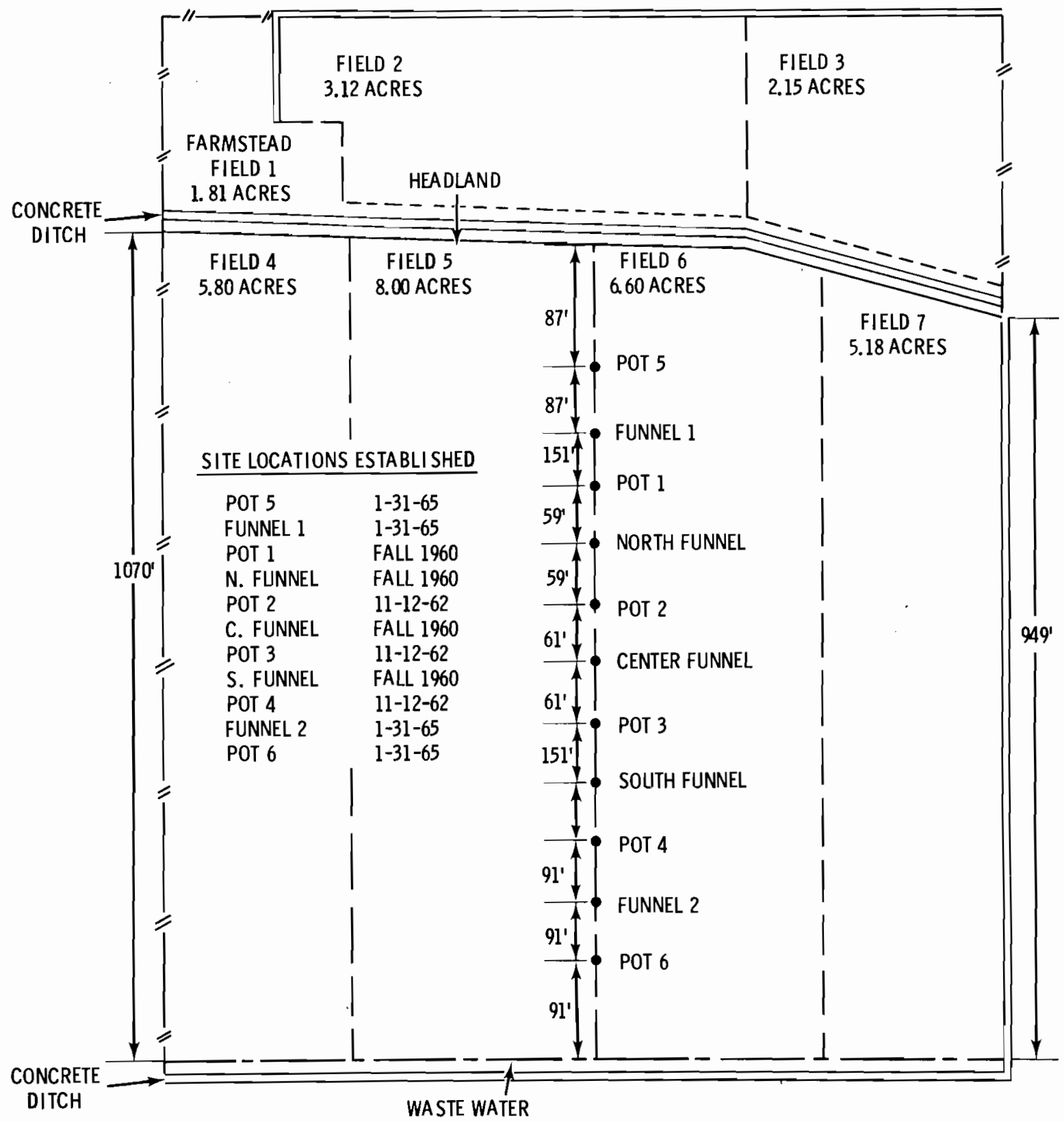

FIGURE B3. Spatial Arrangement of "Pot and Funnel" Fallout Collectors on the Syphus Dairy Farm 


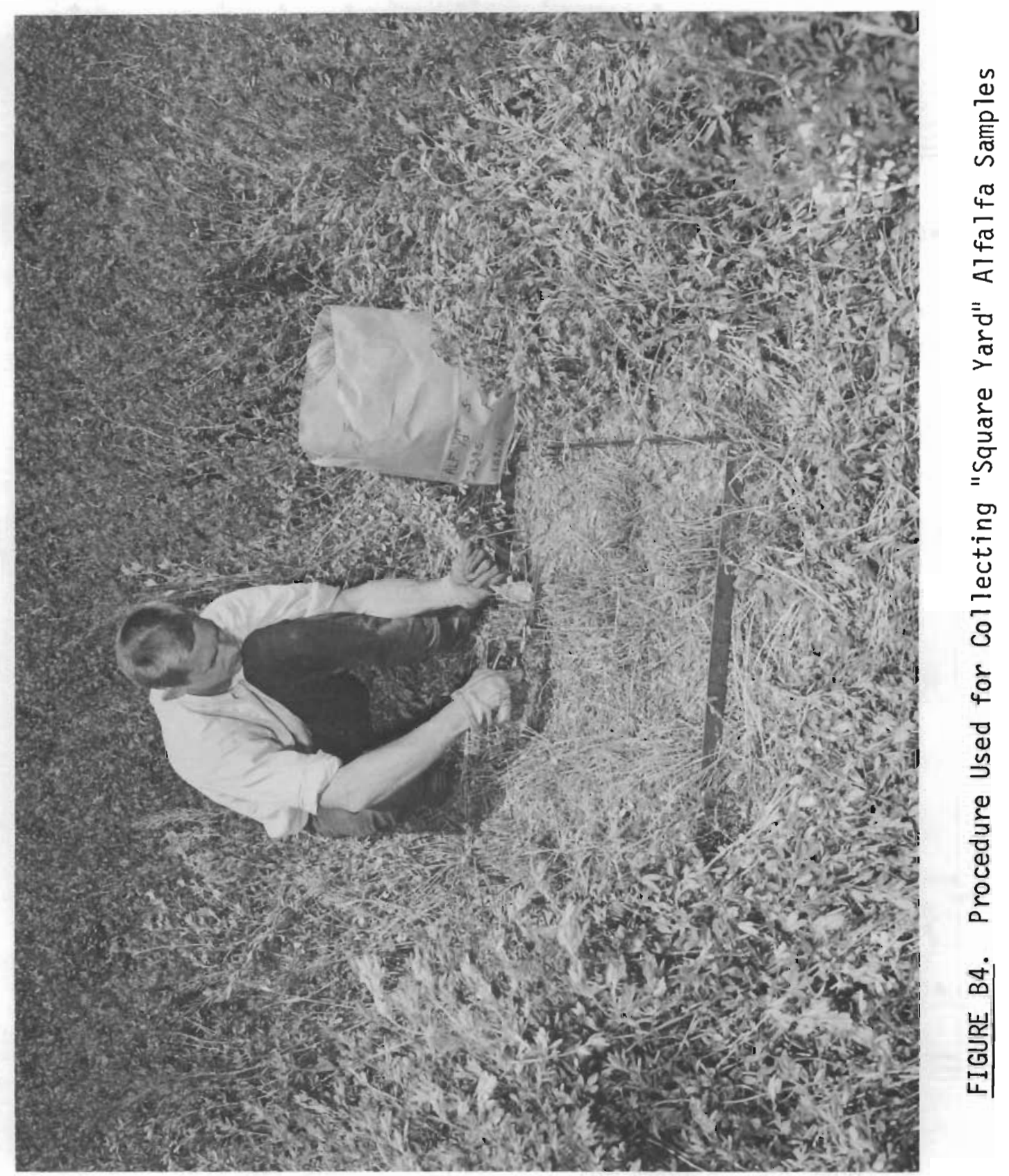

B. 3 
LEO SYPHUS FARM

SAINT GEORGE, UTAH

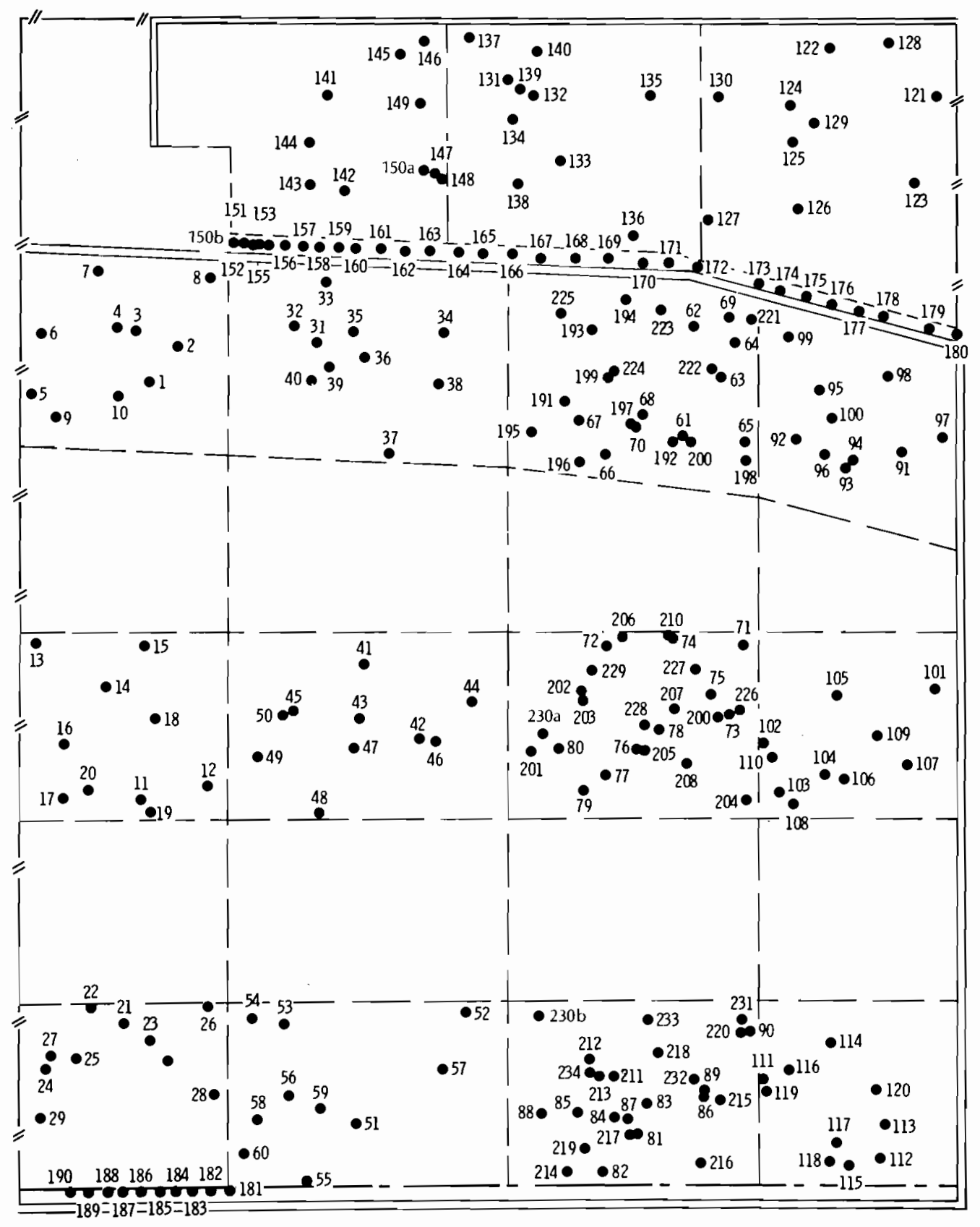

FIGURE B5. Random Soi1 Sampling Stations 1962-1963 


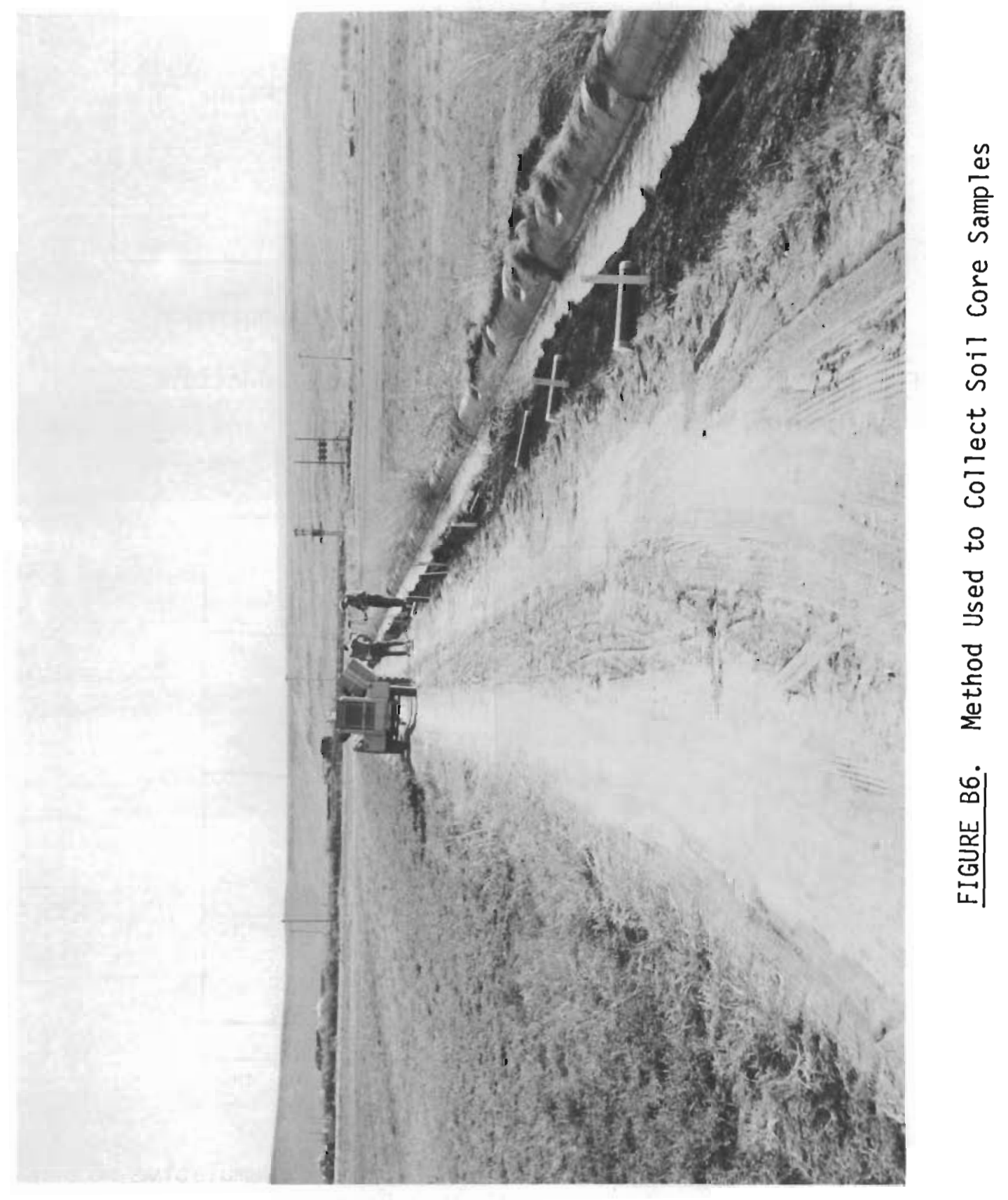

B. 5 

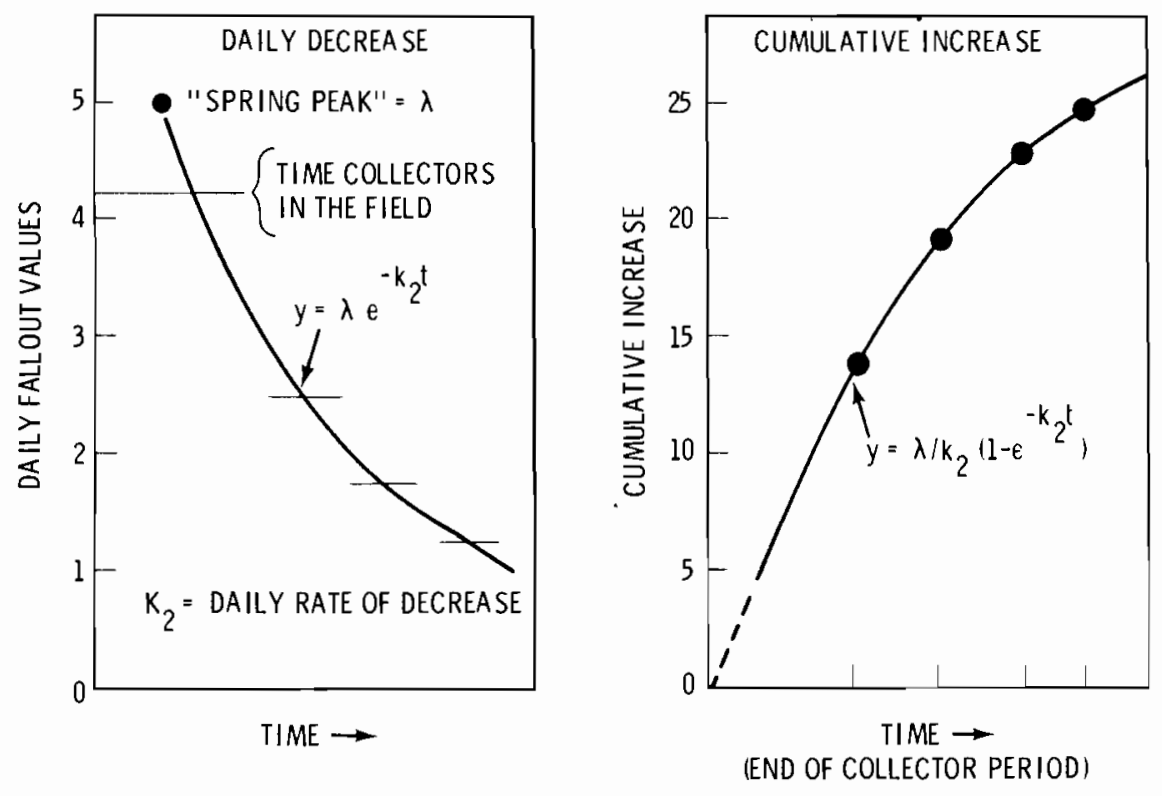

FIGURE B7. Models for Contents of Fal lout Collectors

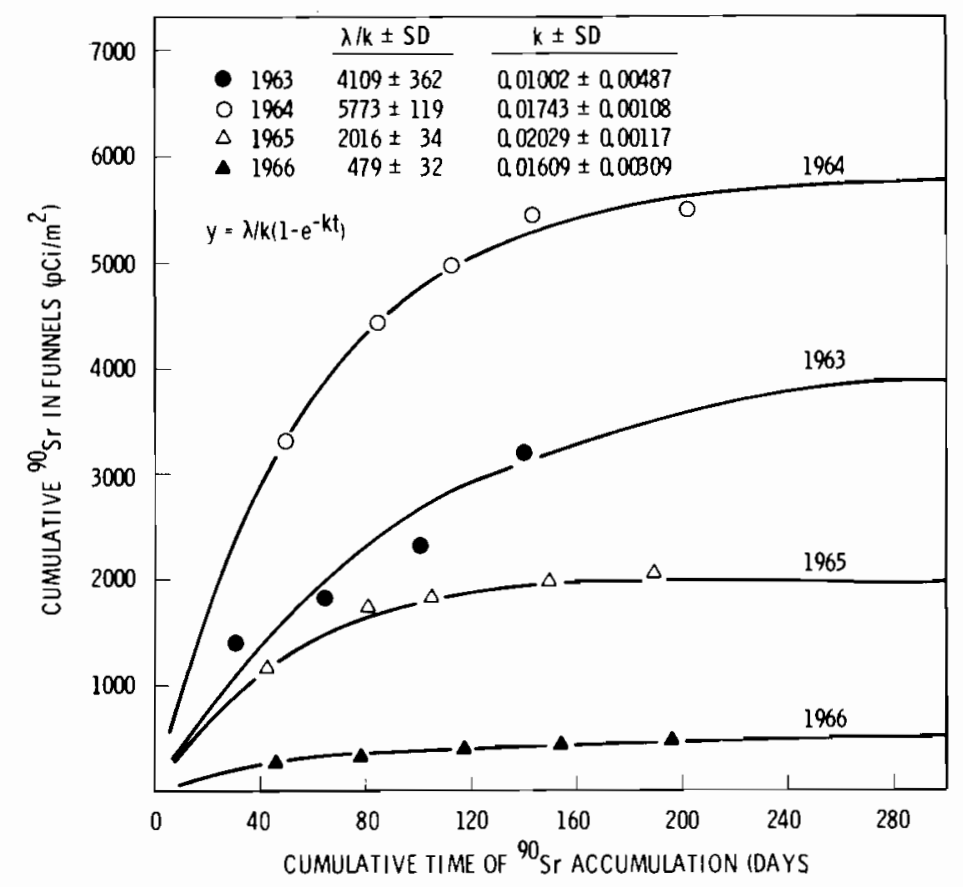

FIGURE B8. Nonlinear Least-Squares Fits of Cumulative "Funnel" ${ }^{90} \mathrm{Sr}$ Collector Data - Each Year Separately 


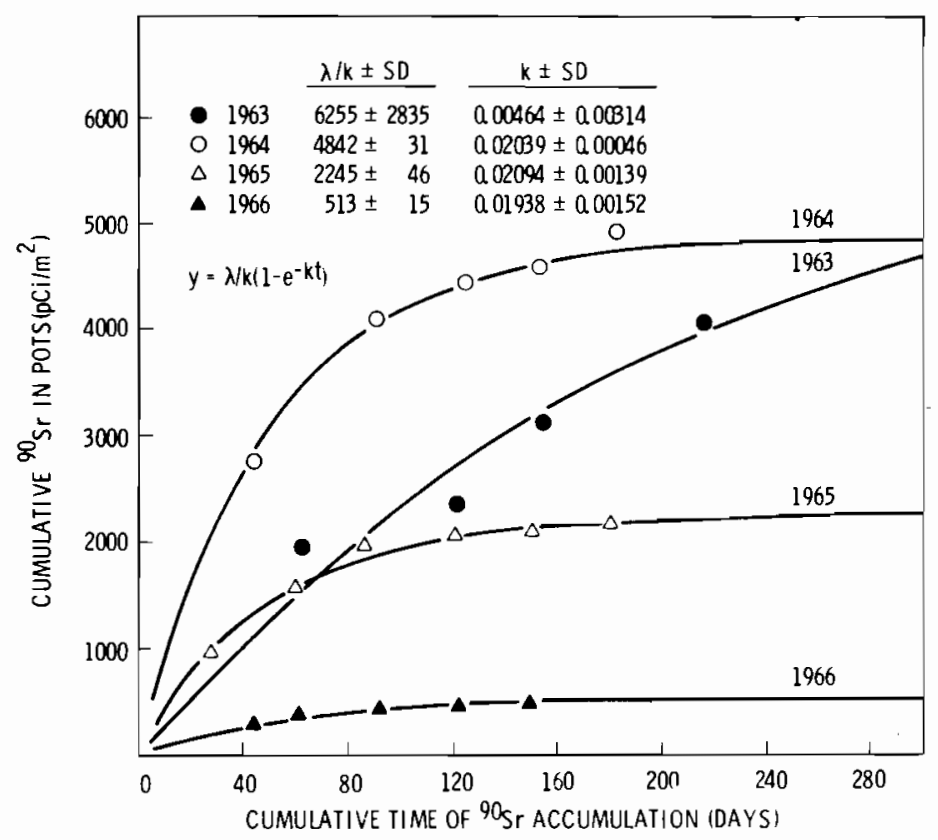

FIGURE B9. Nonlinear Least-Squares Fits of Cumulative "Pot" $90 \mathrm{Sr}$ Collector Data - Each Year Separately

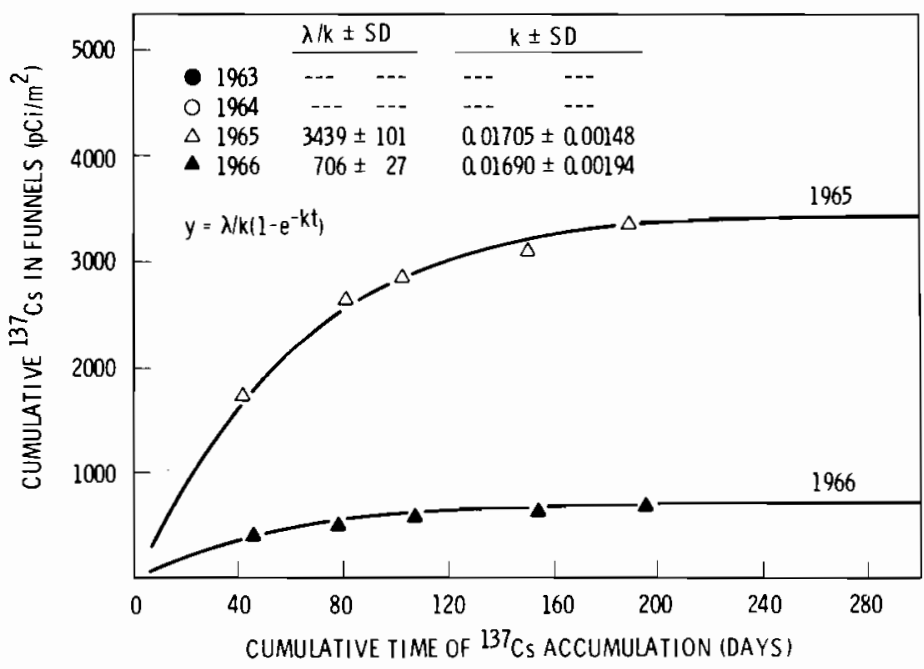

FIGURE B10. Nonlinear Least-Squares Fits of Cumulative "Funne1" 137 Cs Collector Data - Each Year Separately 


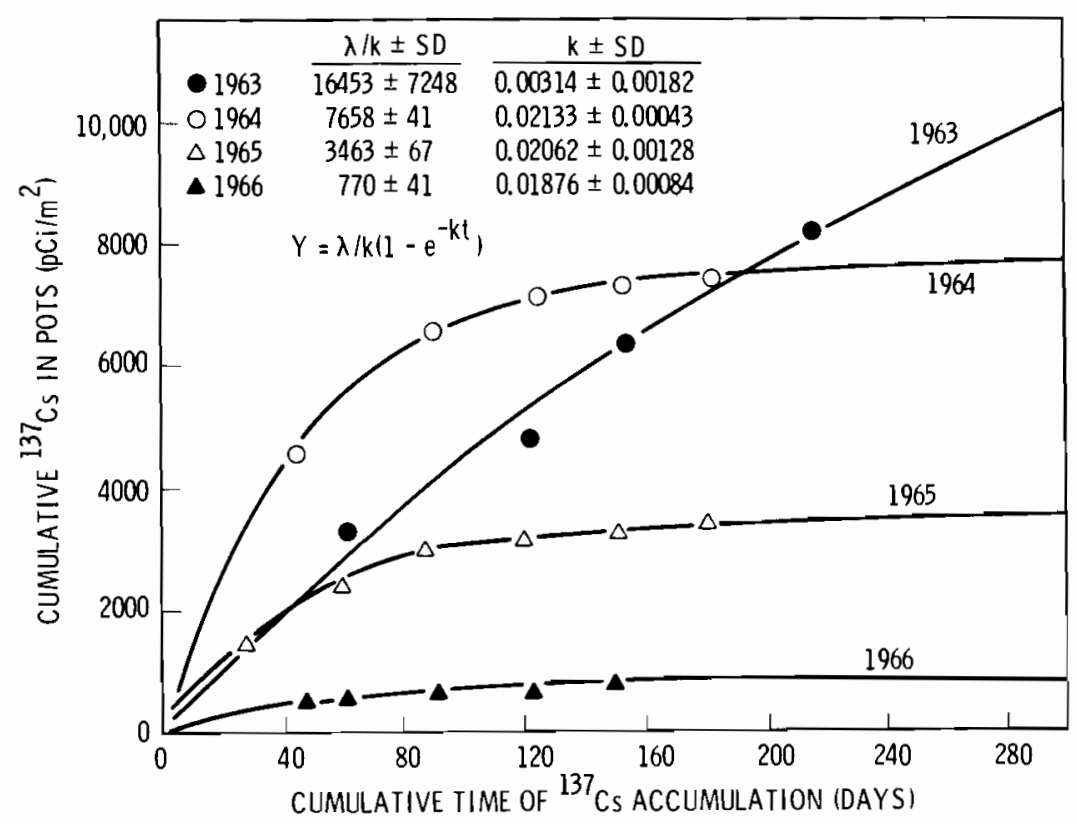

FIGURE B1 1. Nonlinear Least-Squares Fits of Cumulative "Pot" ${ }^{137} \mathrm{Cs}$ Collector Data - Each Year Separately

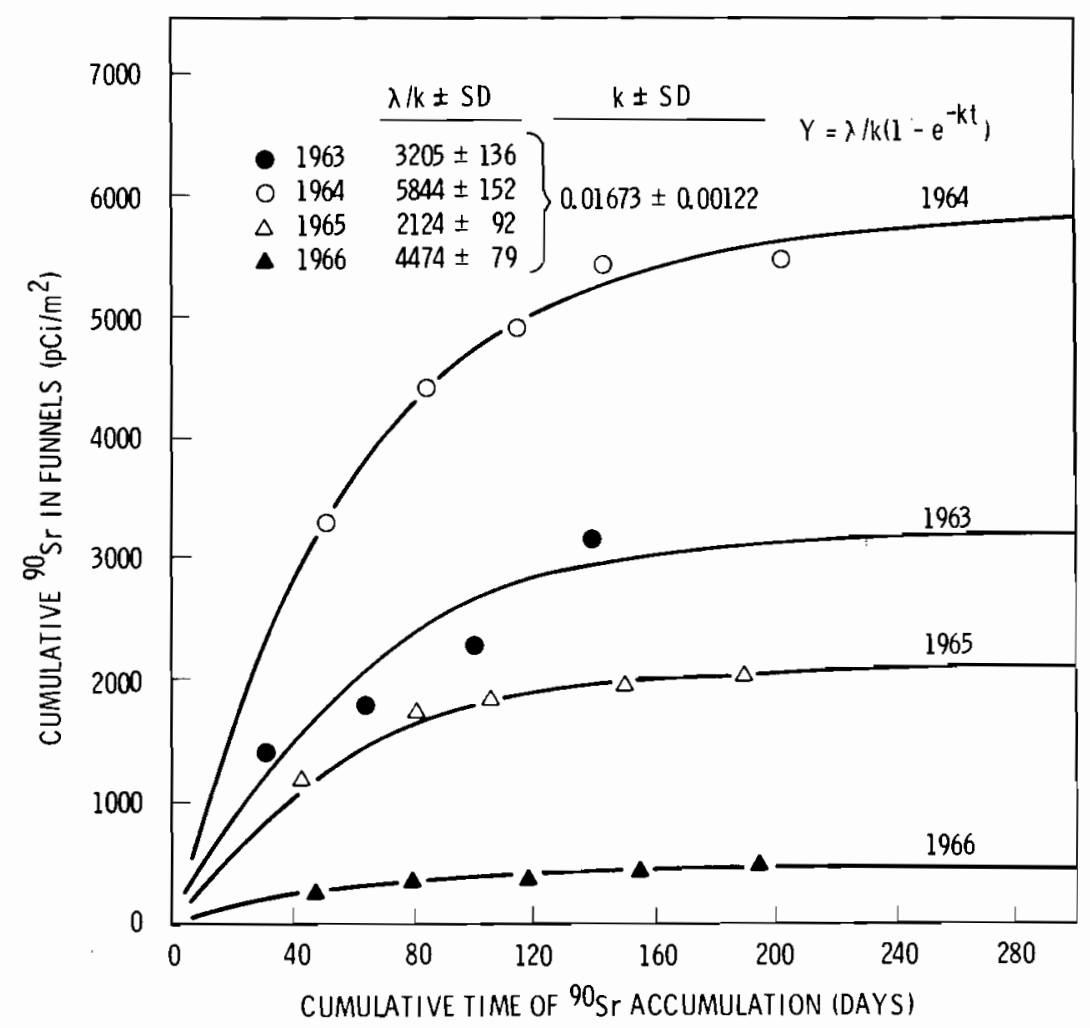

FIGURE B12. Nonlinear Least-Squares Fits of Cumulative "Funnel" 90 Sr Collector Data - Joint Fit 


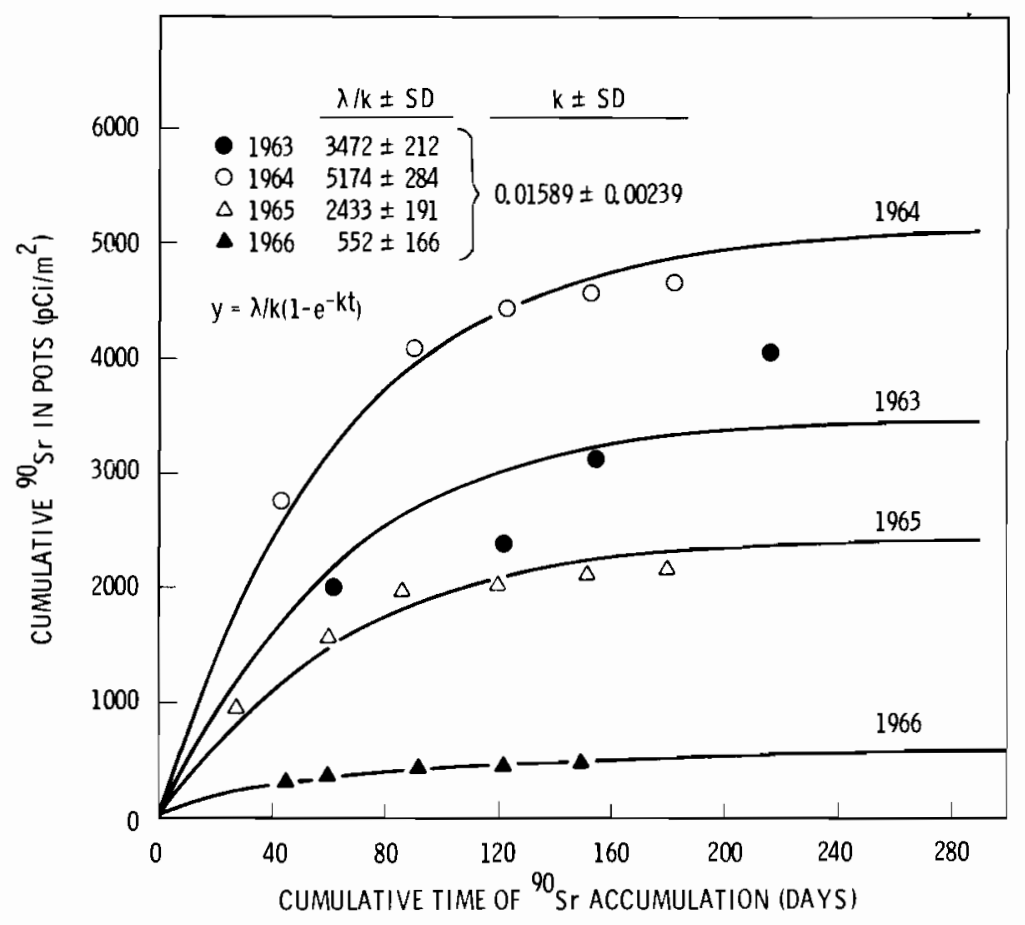

FIGURE B13. Nonlinear Least-Squares Fits of Cumulative "Pot" 90Sr Data Joint Fit

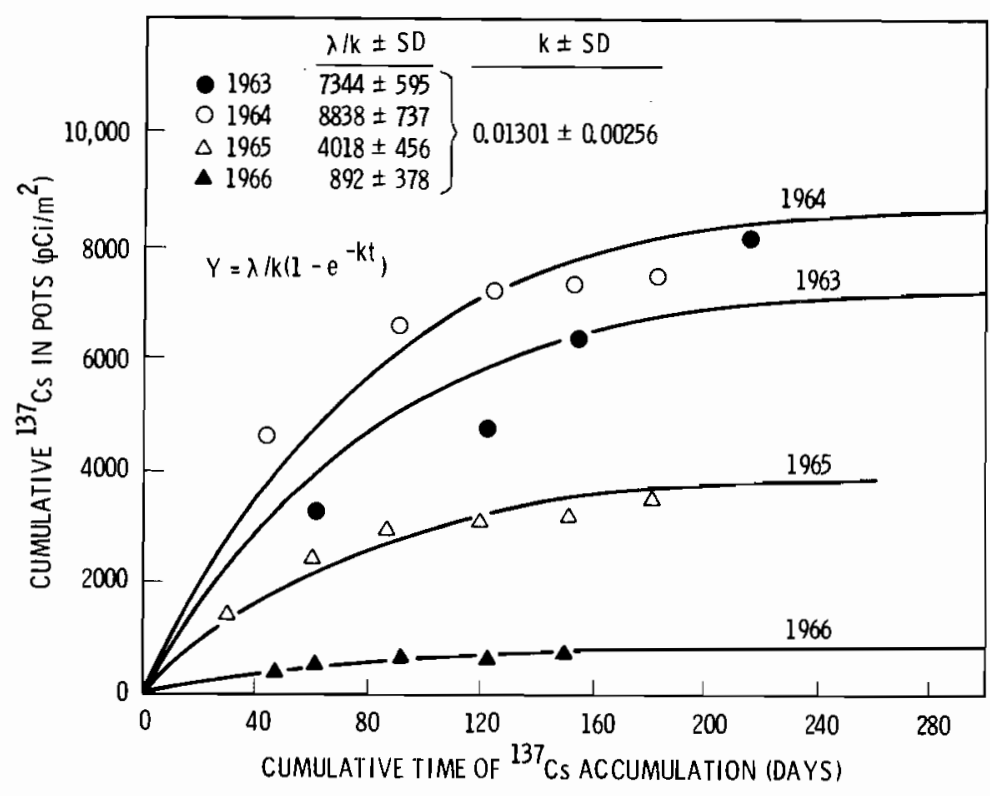

FIGURE B14. Nonlinear Least-Squares Fits of Cumulative "Pot" ${ }^{137} \mathrm{Cs}$ Collector Data - Joint Fit 


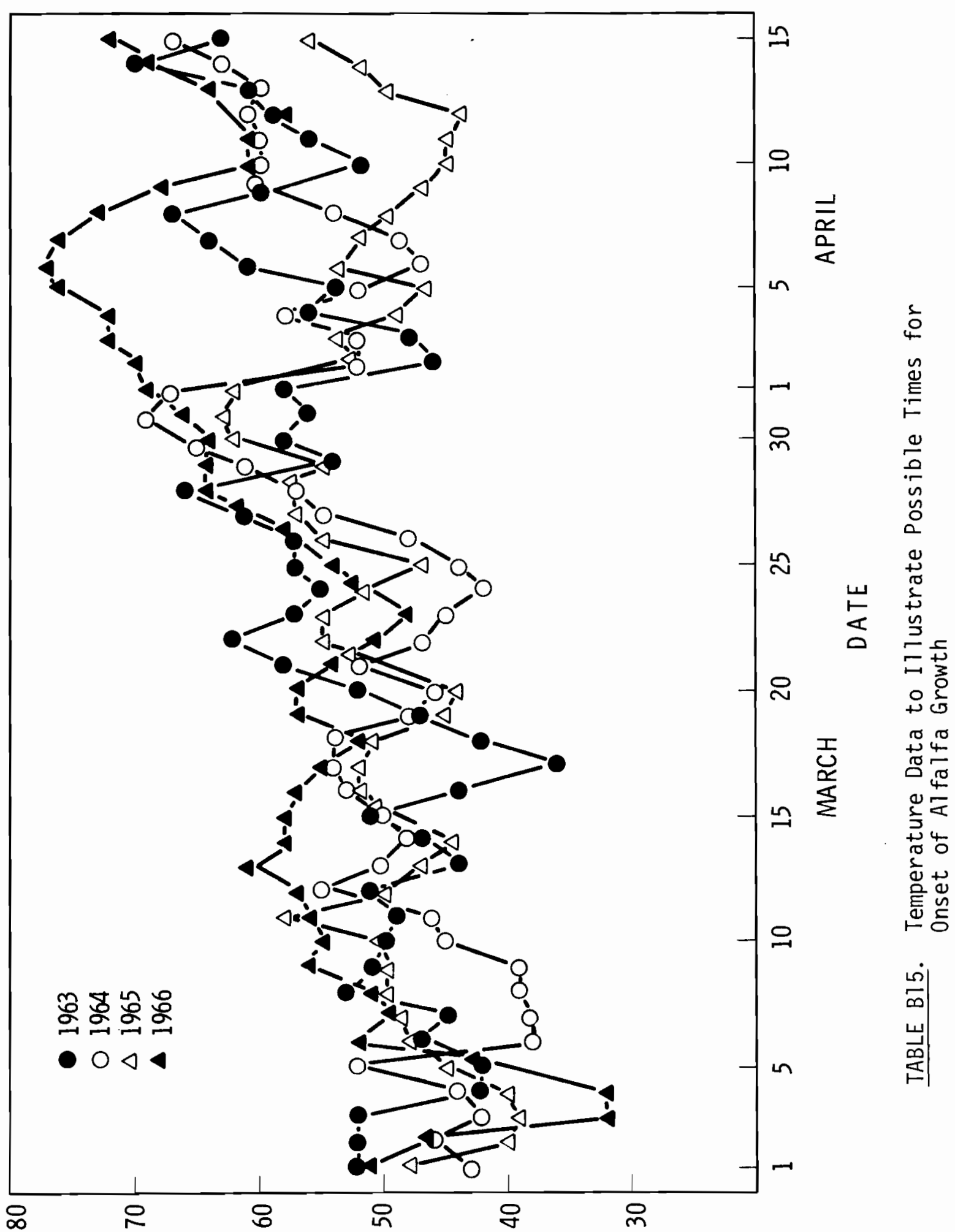

$\left(\exists_{0}\right) \exists y \cap \perp \forall y \exists d W \exists \perp \wedge 7 I \forall O N \forall \exists W$ 


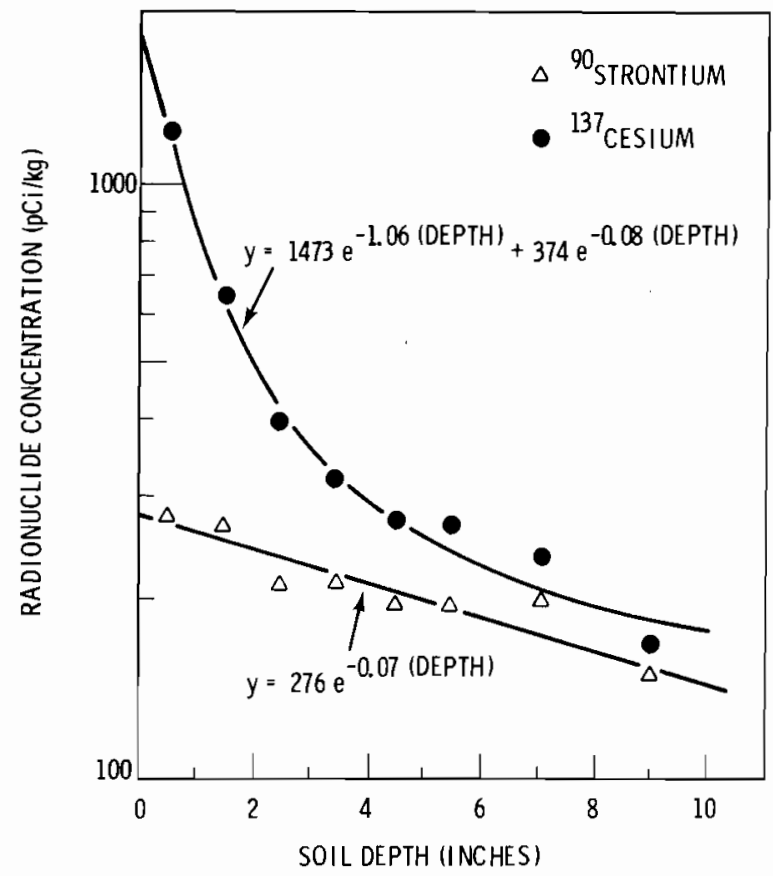

FIGURE B16. $\quad{ }^{137}$ Cesium and ${ }^{90}$ Strontium Concentrations in the Soil Profile (1965 Data)

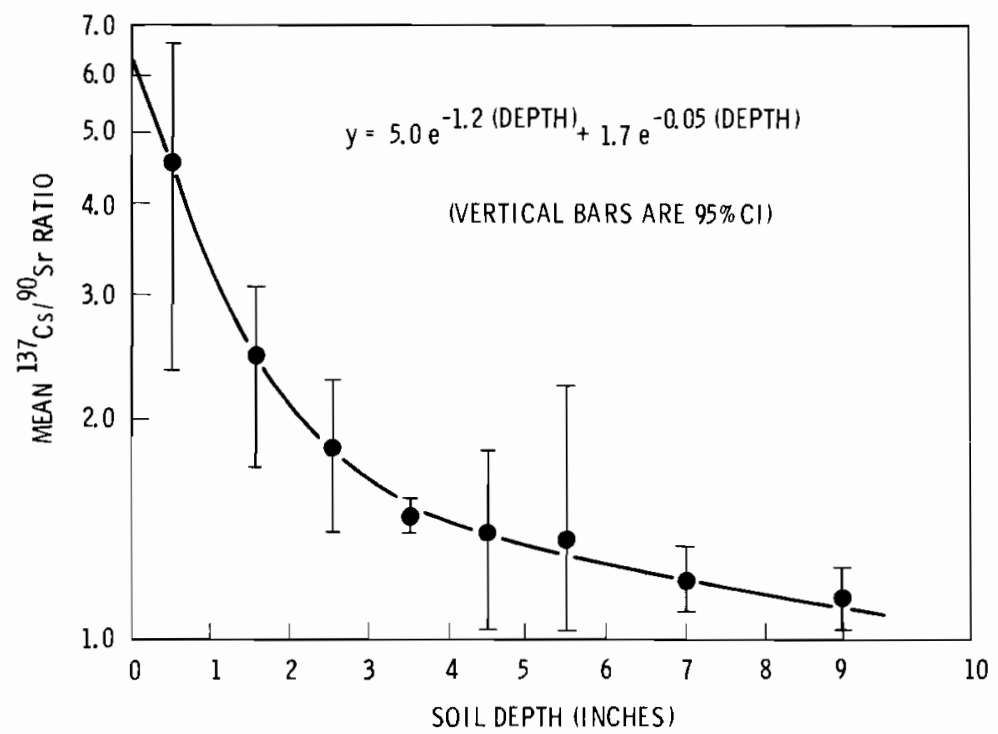

FIGURE B17. $\quad{ }^{137}$ Cesium/90 Strontium Ratios in the Soil Profile (1965 Data) 


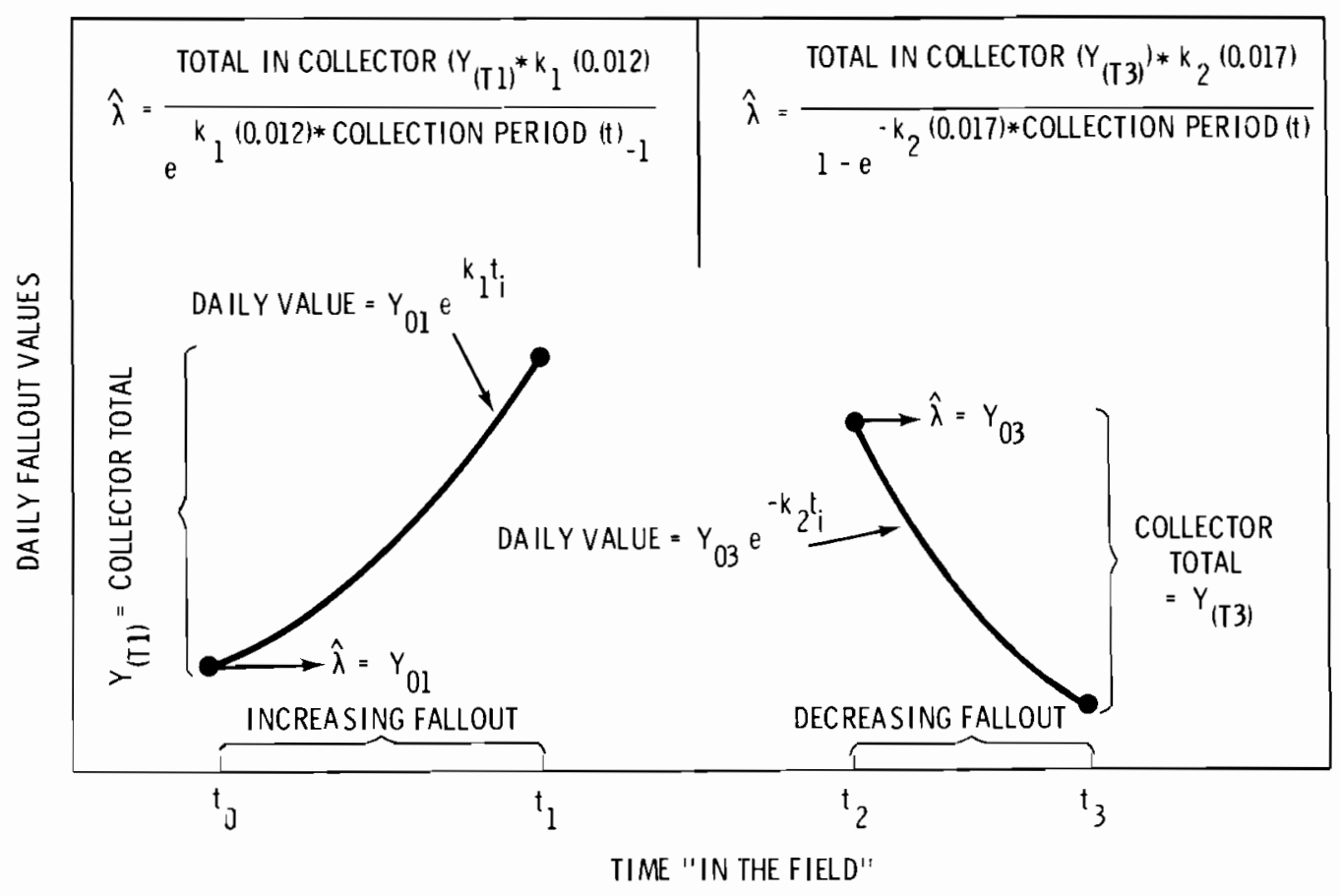

FIGURE B18. Examples of the Calculation of Daily Fallout Values

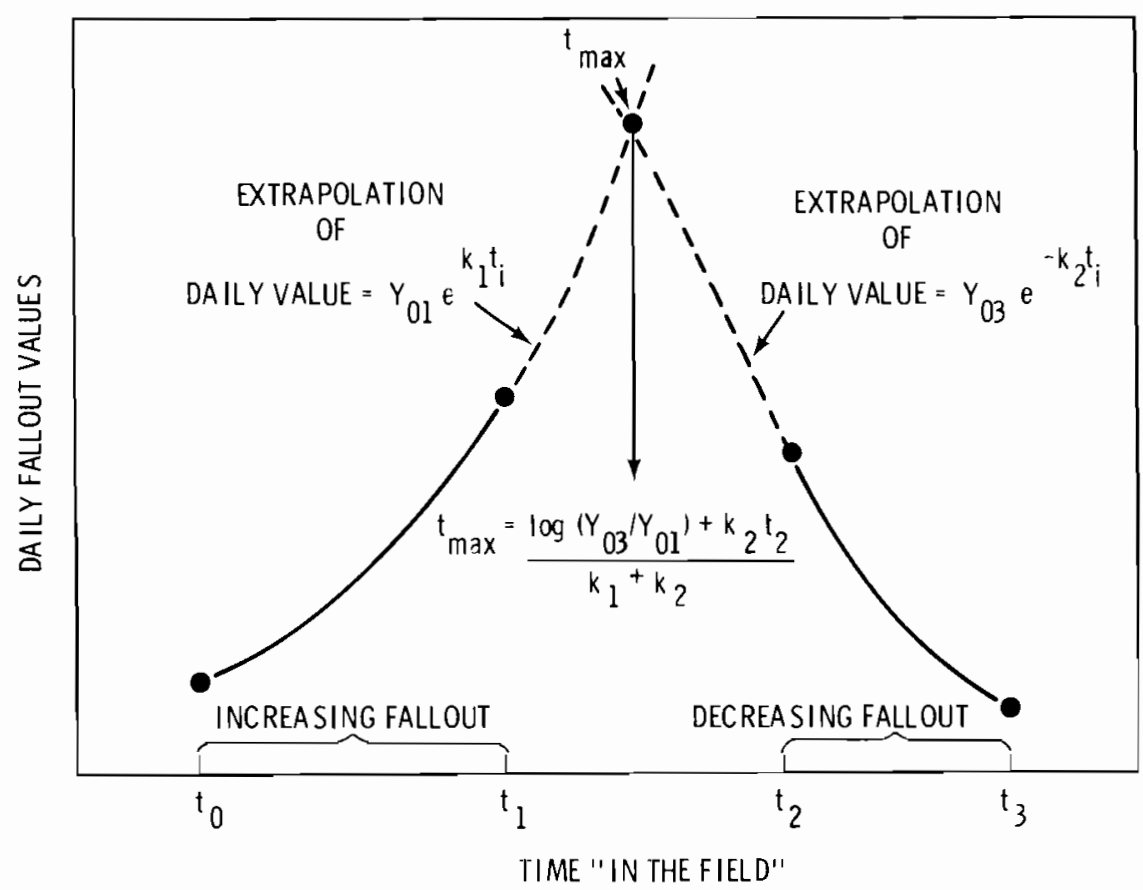

FIGURE B19. Calculation of Time of "Spring Peak" of Fallout - $t_{\text {MAX }}$ 


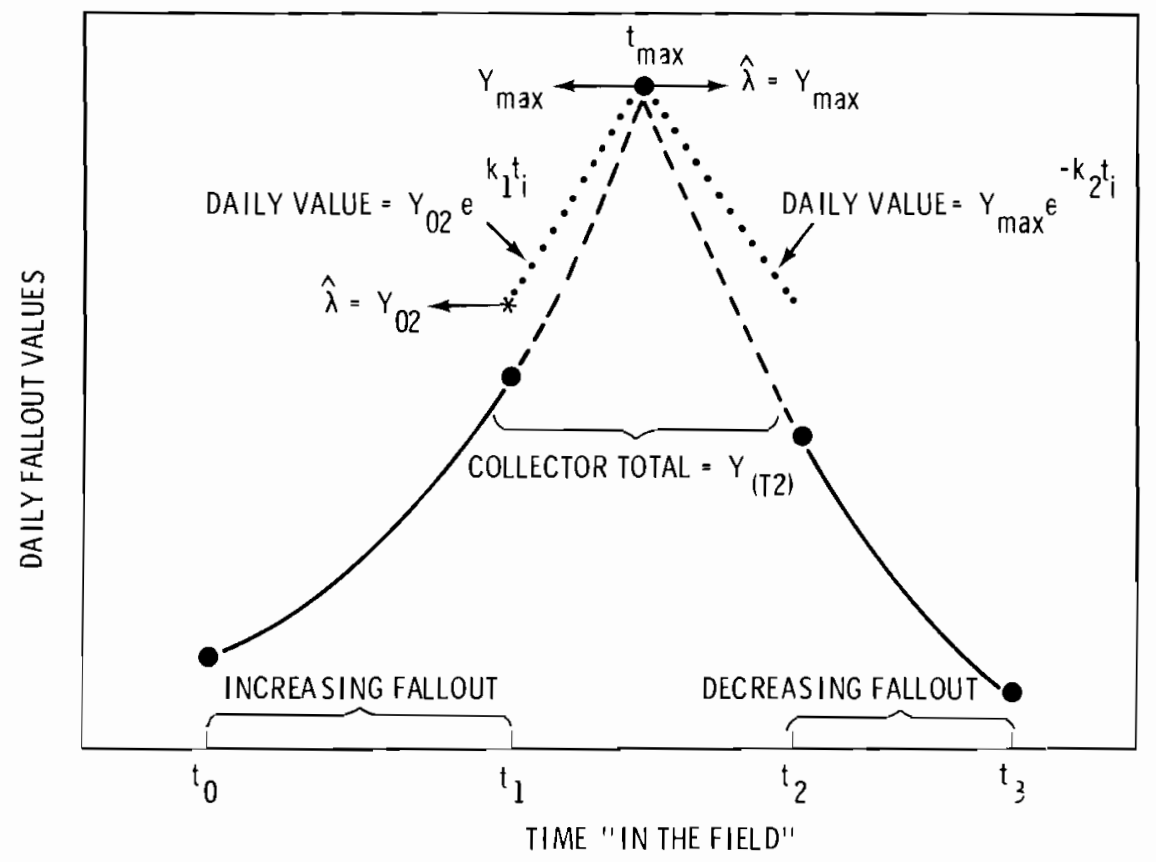

FIGURE B20. Apportionment of "Spring Peak" Collector Total *See (Thomas et. al., 1975)

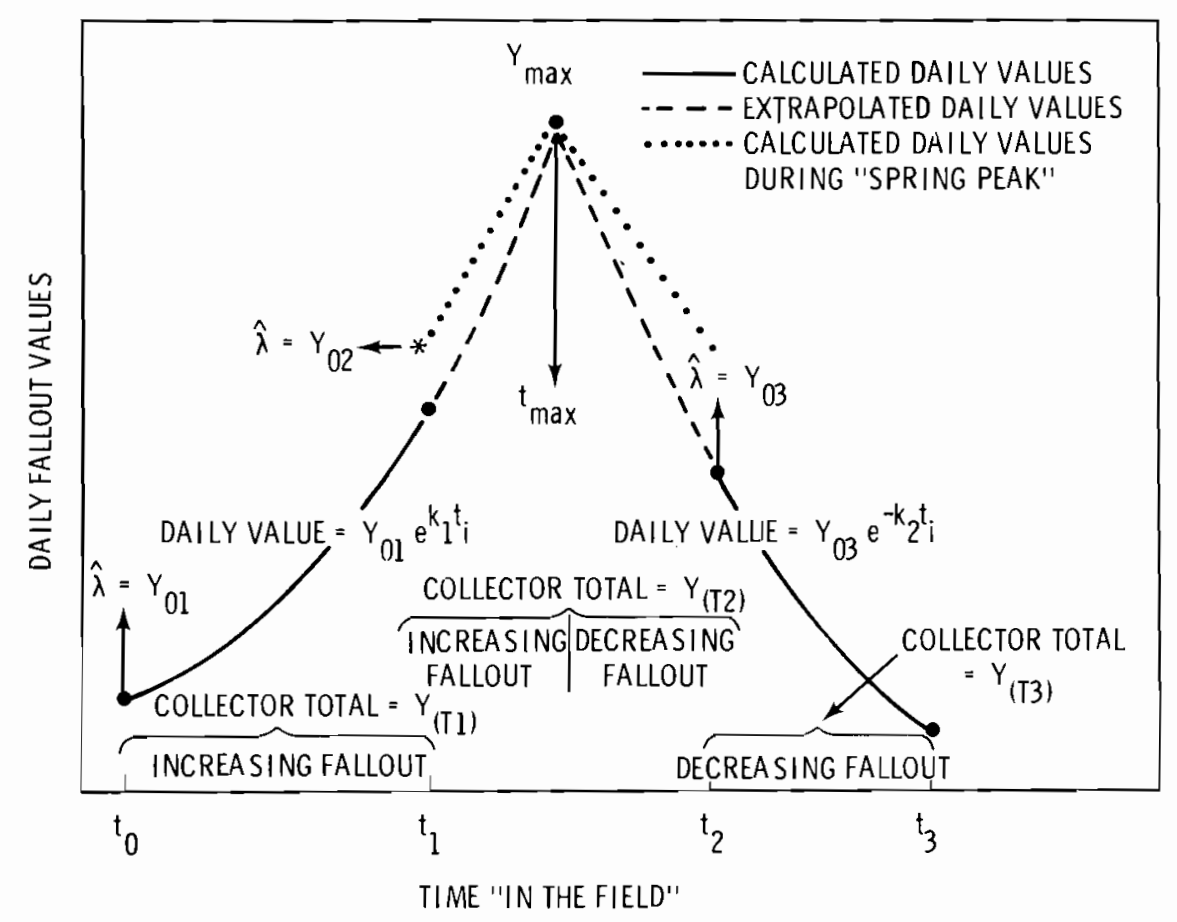

FIGURE B21. Complete Calculation of Daily Fallout Inputs to Alfalfa Plants 


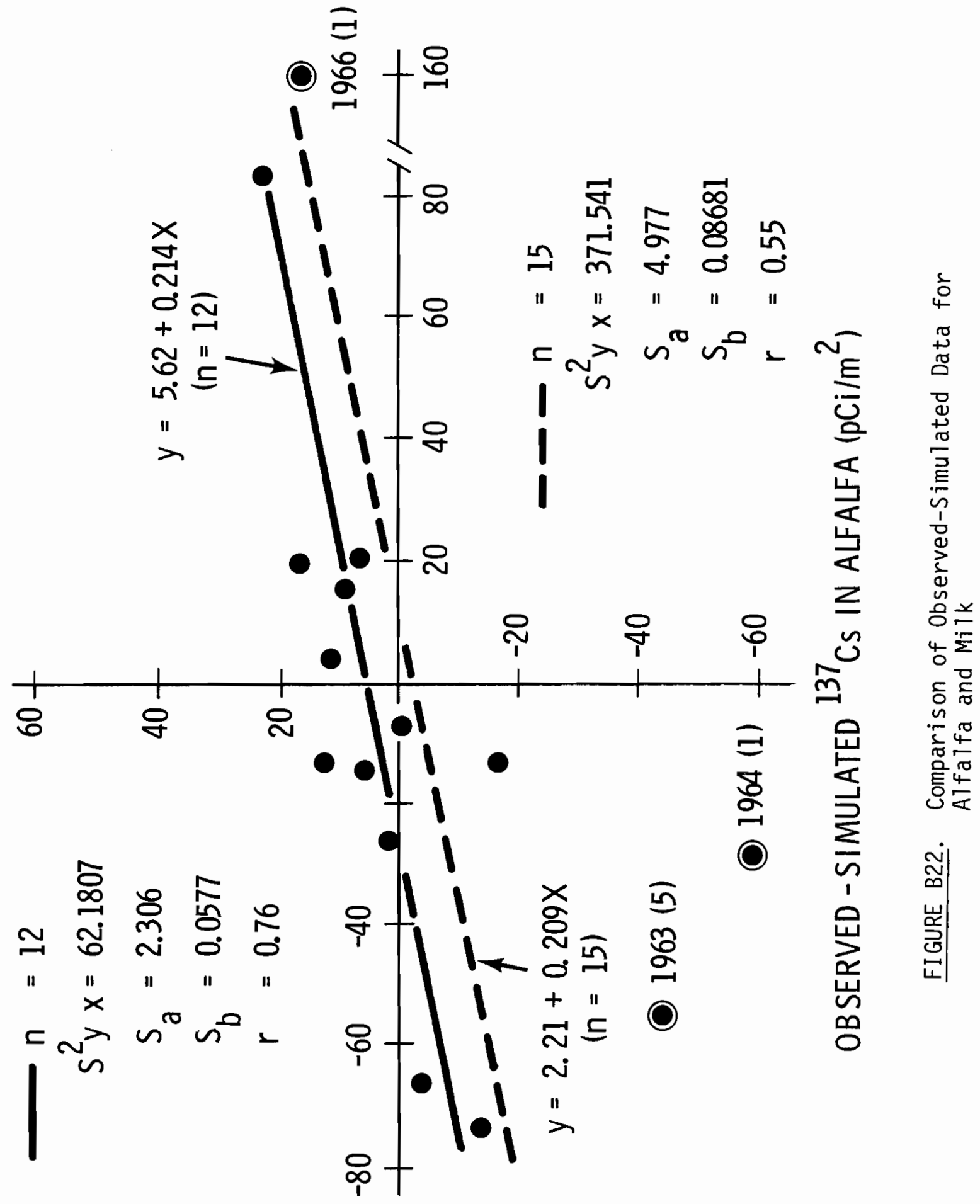

(by/!)d) XITIW NI Sว 


\section{DISTRIBUTION}

No. of Copies

Offsite

1 ERDA Chicago Patent Group Chicago Operations Office 9800 South Cass Avenue Argonne, IL 60439

A. A. Churm

27 ERDA Technical Information Center

5 ERDA Division of Biomedical and Environmenta 7 Research Washington, D. C. 20545

N. F. Barr

R. Franklin

J. Swinebroad

R. L. Watters

C. L. Osterberg

1 ERDA Savannah River Operations Office

Drawer $E$

Aiken, SC 29801

I. L. Brisbin

10 General Electric Co. TEMPO

816 State Street

Santa Barbara, CA 93101

H. A. Hawthorne

3 Division of Energy and Environmental Sys tems

Argonne National Laboratory

Argonne, IL 60439

S. D. Ze17mer
No. of Copies

1 ERDA Idaho Operations Office Idaho Fa17s, ID 83407

P. G. Voillequé

1 Nuclear Regulatory Commission 7920 Norfolk Ave. Bethesda, MD 21230

C. A. Pelletier

1 Electric Power Research Institute P. 0. Box 10412 Palo Alto, CA 94304

C. L. Comar

7 University of California Laboratory of Nuclear Medicine and Radiological Biology Division of Environmental Biology 900 Veterans Avenue Los Angeles, CA 90024

T. L. Hartsock

E. B. Edney

Arthur Wallace

0 . R. Lunt

R. A. Wood

H. Nishita

S. Warren

1 Oak Ridge National Laboratory Ecological Sciences Division Oak Ridge, TN 37830

W. Van Winkle

1 Colorado State University Dept. of Animal Science Ft. Collins, CO 80521

G. M. Ward 
1 Colorado State University

Dept. of Statistics

Ft. Collins, CO 80521

E. E. Remmenga

1 Dames and Moore

1414 Dexter Ave. $N$.

Seattle, WA 98109

Kermit Larson

1 University of Cincinnati

Department of Biological Sciences

Cincinnati, $\mathrm{OH} 45221$

Dr. J. C. Beatley

3 General Electric Company-TEMP0

P.0. Box QQ

Santa Barbara, CA 93102

W. W. Chan

K. W. Lauderdale

R. H. Rowland

1 National Science Foundation

Division of Advanced Environmental Research \& Technology

Washington, D.C. 20550

Dr. R. A. Carrigan

1 D. F. Humphrey

2155 Brown Road, N.W.

Salem, Oregon 97303

1 J. F. Irwin

40053 N. 90th Street West

Leona Valley, CA 93550

1 F. M. Plesset

1180 Amalfi Drive

Pacific Palisades, CA 90272
1 Dr. R. F. Reitemeier

Apt. 324

350 Paseo De Playa

Ventura, CA 93001

1 Dr. J. F. Ross

32-139 Center for Health Sciences

University of California

Los Angeles, CA 90024

1 W. J. Siembab, Jr.

814 S. Shenandoah

Los Angeles, CA 90403

1 E. E. Syphus

233 South 600 East

St. George, Utah 84770

ONSITE

3 ERDA Richland Operations Office Environmental \& Laboratory

Programs Division

Paul F. X. Dunigan, Jr.

Office of the Manager

A. G. Fremling

Battelle-Northwest

L. L. Eberhardt (12)

John M. Thomas (12)

R. L. Wildung

R. L. Hooper

M. I. Cochran

R. 0. Gilbert

J. K. Soldat

Technical Information Library (3)

Technical Publications (1) 